\title{
First Quarter Report of Temperature Measurements During the Heating Phase of the Drift-Scale Test
}

\author{
S. Blair
}

W. Lin

June 1998

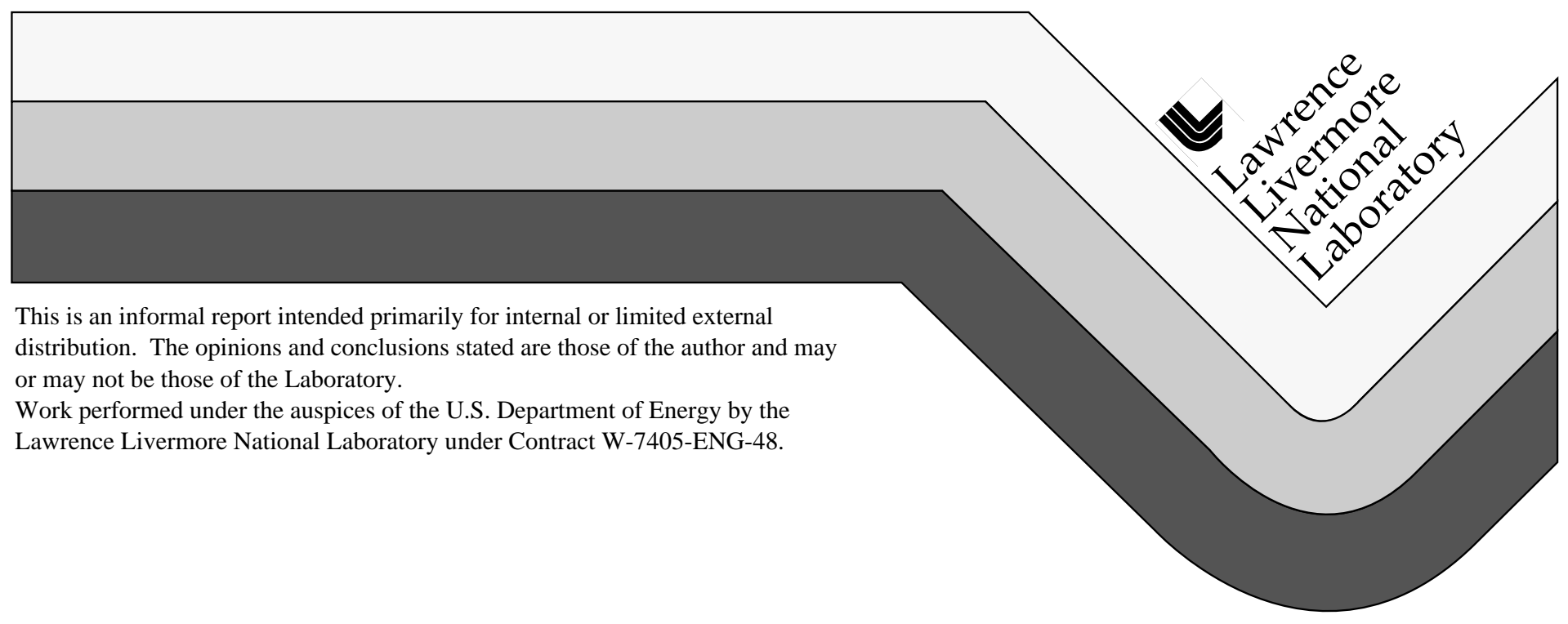




\section{DISCLAIMER}

This document was prepared as an account of work sponsored by an agency of the United States Government. Neither the United States Government nor the University of California nor any of their employees, makes any warranty, express or implied, or assumes any legal liability or responsibility for the accuracy, completeness, or usefulness of any information, apparatus, product, or process disclosed, or represents that its use would not infringe privately owned rights. Reference herein to any specific commercial product, process, or service by trade name, trademark, manufacturer, or otherwise, does not necessarily constitute or imply its endorsement, recommendation, or favoring by the United States Government or the University of California. The views and opinions of authors expressed herein do not necessarily state or reflect those of the United States Government or the University of California, and shall not be used for advertising or product endorsement purposes.

This report has been reproduced directly from the best available copy.

Available to DOE and DOE contractors from the Office of Scientific and Technical Information P.O. Box 62, Oak Ridge, TN 37831

Prices available from (423) 576-8401

Available to the public from the National Technical Information Service

U.S. Department of Commerce 5285 Port Royal Rd. Springfield, VA 22161 


\title{
First Quarter Report of Temperature Measurements During the Heating Phase of the Drift-Scale Test
}

\author{
Milestone SP2640M4 \\ Stephen Blair and Wunan Lin
}

\section{Introduction}

The Drift-Scale Test (DST) is a thermal test being conducted in the Exploratory Studies Facility (ESF) at Yucca Mountain, Nevada. One of the DST's major objectives is to study the coupled thermal-mechanical-hydrologic-chemical processes at the repository horizon of a potential high-level nuclear waste repository at Yucca Mountain. The objectives, the test design, and the test layouts of the DST are included in the test design report by CRWMS $\mathrm{M} \& \mathrm{O}$ Contractor (CRWMS M\&O, 1996).

The purpose of this report is to present temperature data collected during the first quarter of DST heating. This report presents a brief discussion of the layout of the DST and design of the temperature measurements. The overall thermal response of the rock during the reporting period is presented by discussing temperature data, as a function of location and time for several of the boreholes used for temperature measurements. Also presented is a preliminary comparison of one vertical temperature profile observed at the end of the first quarter with a predicted temperature profile. In addition, relative humidity and barometric pressure data for the first quarter are presented and discussed, along with an evaluation of the temperature sensors' performance.

\section{DST Layout and Temperature Measurements}

The configuration of the DST (see Figure 1) includes a declining access / observation drift (AOD), at about $2.827 \mathrm{~km}$ from the north portal driven mostly east and downward from the main tunnel in the ESF. The downward slope (11.5 to 14.0\%) of the AOD ensures a minimum $10 \mathrm{~m}$ of middle nonlithophysal Topopah Spring tuff as the overburden for the DST. The length of the observation drift is approximately $136 \mathrm{~m}$. At the elevation of the DST crown (nominally $10 \mathrm{~m}$ below the upper extent of the middle nonlithophysal Topopah Spring tuff), the connecting drift breaks out to the north from the observation drift, $136 \mathrm{~m}$ from the main tunnel of the ESF. The connecting drift extends approximately $40 \mathrm{~m}$ northward from the observation drift. A heater drift breaks out westward from the connecting drift at approximately $30 \mathrm{~m}$ from the AOD. The heater drift consists of an 11-m long entry, which includes a plate-loading niche, and a 47-m long heated drift (HD). The nominal diameter of the drifts is $5 \mathrm{~m}$. The detailed configuration of the DST, including diagrams showing the drift and borehole layout, is included in the test design report by CRWMS M\&O Contractor (CRWMS M\&O, 1996).

The rock mass being monitored in the DST is being heated by electrical heaters placed on the invert of the HD and in horizontal wing-heater boreholes. The layout of the heaters is shown in Figure 2. Scoping model calculations have been conducted to determine heater 
specifications such as power output and duration (Birkhozer and Tsang, 1997; Buscheck and Nitao, 1995; Buscheck et al., 1997). Criteria for determining the heater specifications include the maximum temperature in the drift wall, the volume of the rock mass to be heated, and the volume of rock mass to be dried by heating. The heating system includes, in addition to the heaters, a means to control heater output in accordance with predetermined heating/cooling schedules.

The heated section of the HD is nominally $47.5 \mathrm{~m}$ long and houses 9 floor heaters along the centerline of the HD. Fifty wing heaters are uniformly spaced in 11.5-m boreholes drilled into the 2 side walls of the HD (Figure 2). The total heat output available for the floor and wing heaters is approximately $68 \mathrm{~kW}$ and $143 \mathrm{~kW}$, respectively. The test design calls for four years of heating, which calculations indicate will create a drying zone around the HD extending approximately $10 \mathrm{~m}$ into the surrounding rock. During the heating phase, driftwall temperatures should not exceed $200^{\circ} \mathrm{C}$.

Heating of the DST was started on December 3, 1997, and electrical power to the heaters has been monitored and is reported elsewhere (SNL, 1998). This monitoring showed that, during the first quarter of heating, $136 \mathrm{~kW}$ of power was supplied to the wing heaters and $52 \mathrm{~kW}$ of power was supplied to the canister (floor) heaters. Temperatures of the canister and wing heaters and of the air in the HD are also being monitored along with the temperature of the upper portion of the drift wall in the HD. These temperature data are reported elsewhere (SNL, 1998). Air temperatures after 87 days of heating are $100 \pm 5^{\circ} \mathrm{C}(\mathrm{SNL}, 1998)$.

This report presents temperature data through February 28, 1998, for temperatures monitored in the rock mass. Rock mass temperatures in the DST are being measured primarily using resistance temperature device (RTD) sensors in several boreholes. These boreholes are tabulated in Table 1, and the approximate locations of these holes are shown in Figure 3.

Temperature is also monitored in three cross sections oriented perpendicular to the axis of the drift. These cross sections provide radial coverage of the temperature field around the DST. They are located at approximately 12, 23, and $39 \mathrm{~m}$ from the bulkhead. Table 1 shows that temperature in the cross sections at 12 and $23 \mathrm{~m}$ are being monitored using 8 boreholes, while temperature in the cross section at $39 \mathrm{~m}$ is monitored using 6 boreholes. The relative geometry of boreholes in these cross sections is shown in Figures $4 \mathbf{a}, \mathbf{4 b}$, and $4 \mathbf{c}$.

Two other temperature boreholes (boreholes 79 and 80) are also listed in Table 1 and shown in Figures 3 and 4 . These boreholes are parallel to the axis of the drift and directly above the midplane of the wing heaters on either side of the HD. The elevation of these boreholes is above the drift crown as shown in Figure 3. The purpose of these boreholes is to (1) monitor temperature in the region where long-term boiling is expected, (2) assess the heterogeneity in temperature along the axis of the drift, and (3) monitor temperatures at the ends of the heated zone for estimation of end effects.

The as-built xyz coordinates of the collar and bottom of those boreholes are provided by the Test Coordinating Office with a data-tracking number LANE834244AQ97.001. These are further documented in deliverable SPY195M4 (Lin and Wagoner, 1998). 
Table 1 Summary of Temperature (RTD) Boreholes

\begin{tabular}{|c|c|c|c|}
\hline $\begin{array}{l}\text { Distance from } \\
\text { Bulkhead }\end{array}$ & $\begin{array}{l}\text { Number of } \\
\text { Boreholes }\end{array}$ & $\begin{array}{c}\text { Borehole } \\
\text { Number }\end{array}$ & $\begin{array}{l}\text { Orientation } \\
\text { (degrees) }\end{array}$ \\
\hline \multirow[t]{2}{*}{$3 \mathrm{~m}$} & \multirow[t]{2}{*}{2} & 133 & 0 \\
\hline & & 134 & 180 \\
\hline \multirow[t]{8}{*}{$12 \mathrm{~m}$} & \multirow[t]{8}{*}{8} & 137 & 0 \\
\hline & & 138 & -45 \\
\hline & & 139 & -90 \\
\hline & & 140 & -135 \\
\hline & & 141 & 180 \\
\hline & & 142 & 135 \\
\hline & & 143 & 90 \\
\hline & & 144 & 45 \\
\hline \multirow[t]{8}{*}{$23 \mathrm{~m}$} & \multirow[t]{8}{*}{8} & 158 & 0 \\
\hline & & 159 & -45 \\
\hline & & 160 & -90 \\
\hline & & 161 & -135 \\
\hline & & 162 & 180 \\
\hline & & 163 & 135 \\
\hline & & 164 & 90 \\
\hline & & 165 & 45 \\
\hline \multirow[t]{2}{*}{$32 \mathrm{~m}$} & \multirow[t]{2}{*}{2} & 168 & 0 \\
\hline & & 169 & 180 \\
\hline \multirow[t]{6}{*}{$39 \mathrm{~m}$} & \multirow[t]{6}{*}{6} & 170 & 0 \\
\hline & & 171 & -45 \\
\hline & & 172 & -135 \\
\hline & & 173 & 180 \\
\hline & & 174 & 135 \\
\hline & & 175 & 45 \\
\hline \multirow[t]{2}{*}{ N/A } & \multirow[t]{2}{*}{2} & 79 & 0 \\
\hline & & 80 & 0 \\
\hline
\end{tabular}

$0^{\circ}$ indicates vertical borehole in the roof of the HD. $180^{\circ}$ indicates vertical borehole in the floor of the HD.

Angles are measured clockwise when looking into the HD from the bulkhead (see Figure 4). 


\section{Temperature Observations: December 3, 1997-February 28, 1998}

\subsection{Overview of Temperature Field}

Heating of the DST began on December 3, 1997, and continued uninterrupted throughout the reporting period. Temperature data for the radial RTD boreholes on February 28, 1998, are shown in a perspective plot in Figure 5. Borehole locations are viewed looking toward the bulkhead from a point beyond the end of the HD. This plot shows that, as expected, temperatures are highest near the drift wall and in the plane of the wing heaters. The temperature field appears to be uniform along the length of the HD and is more or less symmetric about the vertical plane along the drift axis. Temperatures at the top of the drift and along the wing heaters are at or slightly above the boiling point of water. In the following subsections, the temperature field is discussed in more detail. Appendix A presents a listing of temperature data collected on selected days for these boreholes.

\subsection{Vertical Profiles Along Drift Axis}

Temperatures in vertical profiles along the drift axis are being monitored at distances of 3 , $12,23,32$, and $37 \mathrm{~m}$ from the bulkhead. At each of these distances, one upward and one downward borehole have been instrumented with RTD temperature sensors along the borehole length. Temperatures observed in these boreholes, after 87 days of heating, are shown in Figures 6 and 7.

Figure 6 shows temperature as a function of vertical position for each of these vertical boreholes. This plot indicates that rock near the top of the drift has reached a temperature of approximately $105 \pm 5^{\circ} \mathrm{C}$, while temperatures in the invert are approximately $10^{\circ} \mathrm{C}$ cooler. Temperature variations among the five profiles appear to be small, indicating that the rock is heating uniformly along the length of the drift between 3 and $39 \mathrm{~m}$ from the bulkhead.

Figure 7 shows temperatures, plotted as a function of distance from the drift axis, on February 28,1998, in these same 10 vertical boreholes. This plot more clearly delineates temperature difference between the top and bottom of the drift. It is important to note that, to minimize temperature differences in the drift, the canister heaters were designed to have a uniform surface temperature. Moreover, the RTD sensors in the downward boreholes are located closer to the canister heaters and should receive more energy by radiation than do those at the top. The difference in temperature between rock at the top and bottom of the drift may be caused by convection in the drift. This is useful data for guiding the numerical modeling of the test because it reflects the relative strength of air convection and radiation as heat-transfer mechanisms.

Figures 6 and 7 also indicate that, on February 28, rock at distances greater than $8 \mathrm{~m}$ from the drift axis was at ambient temperature.

\subsection{End Effects in Axial Direction}

The purpose of boreholes 79 and 80 is to provide temperature information along the axis of the drift in the regions where a boiling front is expected to develop. These boreholes also provide temperature data in the region of high thermal gradients at either end of the HD. 
These measurements are intended to provide constraints on model simulations of the test to minimize and/or identify artifacts caused by end effects. These boreholes were designed to be located above the midpoint of the wing heaters.

Temperature data, as a function of distance from the bulkhead, at several times for borehole 80 are shown in Figure 8. This plot indicates that the area above and to the south of the HD reached approximately $46-48^{\circ} \mathrm{C}$ on February 28, 1998. Temperatures appear highest about $10 \mathrm{~m}$ along the drift axis from the bulkhead and decline slightly with distance along the drift. This figure shows the localized nature of the heating to date, as temperatures drop rapidly in regions outside the interval containing the heaters. Temperatures drop quickly to near ambient on the unheated side of the bulkhead. The lower temperatures at distances beyond $35 \mathrm{~m}$ may be due to deviation of the borehole and/or the liner emplaced at the far end of the HD.

Figure 9 plots temperatures on February 28 for boreholes 79 and 80 together. This plot shows that, at distances as great as $10 \mathrm{~m}$ from the bulkhead (where locations are well known), the temperatures in the two boreholes match well, indicating that the rock in this region is being heated symmetrically about the vertical centerline of the HD. Although this borehole extends more than $40 \mathrm{~m}$ beyond the bulkhead, only RTD locations less than $10 \mathrm{~m}$ beyond the bulkhead are considered reliable.

\subsection{Vertical Cross Section at $12 \mathrm{~m}$ from Bulkhead}

\subsubsection{Vertical and Inclined Boreholes}

Figure 10 shows temperature in boreholes 137 and 141 at a series of times. These boreholes are vertical upward and downward respectively, as shown on the inset. Temperature is plotted as a function of vertical position relative to the axis of the HD. This figure shows that the first few meters of rock above the drift is being heated faster than is rock at the equivalent distance below the drift. This plot shows a smooth increase in temperature with time for this profile and that, at a distance of $10 \mathrm{~m}$ from the HD axis, rock was still at approximately ambient temperature on February 28.

Figure 11 shows temperatures on February 28 for the vertical upward and downward boreholes in this cross section as well as for the boreholes at $\pm 45^{\circ}$ and $\pm 135^{\circ}$. Temperature is plotted as a function of vertical distance from the HD axis.

Boreholes above the HD axis include borehole 137 oriented at $0^{\circ}$ (vertical upward) and boreholes 138 and 144 oriented at $-45^{\circ}$ and $+45^{\circ}$, respectively (see Table 1). Data for these boreholes indicate the following: First, the temperature field above the HD in this cross section is very symmetric about a vertical plane through the axis of the HD. Temperature is highest directly above the HD; temperature distributions vs. distance above the HD axis are identical for the two sides of the HD and are less than $2^{\circ} \mathrm{C}$ lower than the temperatures directly above the drift. This indicates that regions above the wing heaters are being heated evenly and at the same rate as the rock above the HD. This symmetric temperature distribution indicates that, as of February 28, the DST is successfully progressing toward the goal of developing a horizontally planar heated zone above the HD.

Boreholes below the HD axis include borehole 141 oriented at $180^{\circ}$ (vertical downward) and boreholes 140 and 142 oriented at $-135^{\circ}$ and $+135^{\circ}$, respectively (see Table 1 ). Data for these boreholes show a very even temperature distribution in the downward direction, as temperature at a given distance below the plane of the $\mathrm{HD}$ axis varies less than $2^{\circ} \mathrm{C}$ in any of 
the boreholes. Data for these boreholes are consistent with data shown in Figure 7 and indicate that temperatures below the plane of the heaters are lower than those at the same distance above the heaters. This indicates the importance of both convection and conduction as heat transfer mechanisms.

\subsubsection{Horizontal Boreholes}

Figure 12, depicting temperature history for the horizontal boreholes in this cross section (boreholes 139 and 143), shows several interesting features of the temperature in the horizontal plane.

- The temperature field is approximately symmetric, although not as symmetric as observed in the inclined boreholes.

- At distances $<12 \mathrm{~m}$ from the drift, temperature variations as great as $\pm 10^{\circ} \mathrm{C}$ occur along these boreholes. These temperature variations are correlated with the segments of the wing heaters. Lower temperatures are associated with zone between HD and inner segment of wing heater and with the zone between inner and outer wing heater segments.

- Temperature appears to have been higher on the south side (negative $x$ ) of the HD, especially in zones influenced by the outer wing heaters. It is interesting to note that the presence of the AOD on this side of the HD does not appear to influence the temperature distribution.

- Level temperatures at $96^{\circ} \mathrm{C}$ on February 20 and 28 indicate boiling was occurring in significant zones. These zones begin about $5 \mathrm{~m}$ from the drift wall. No evidence of boiling is found near the drift wall. This may indicate that drying has occurred in the first meter or so of rock around the drift wall.

- At distances beyond $14 \mathrm{~m}$ from the drift axis (the end of the wing heaters), temperature declines rapidly back to ambient levels.

Figure 13 shows data from boreholes 139 and 143 as a surface. In this plot, the vertical axis represents temperature, and horizontal distance from the center of the drift is shown across the page. Time from the start of the test in days is shown increasing from the front to the back of the plot. This surface represents a linear interpolation of data presented in Figure 12. Temperatures shown within the $\operatorname{drift}(-2.5 \mathrm{~m}<\mathrm{x}<2.5 \mathrm{~m})$ are not representative.

This plot illustrates the symmetry of the temperature field over time and the persistence of low temperatures in the unheated zones in the wing heater boreholes. The leveling of the temperatures with time in regions of boiling is also shown. Temperature gradients are highest at the sides of the wing heaters.

\subsection{Vertical Cross Section at $23 \mathrm{~m}$ from Bulkhead}

\subsubsection{Vertical and Inclined Boreholes}

Figure 14 shows temperature in boreholes 158 and 162 at a series of times. These boreholes are vertical upward and downward, respectively, as shown on the inset. Temperature is plotted as a function of vertical distance from the axis of the HD. This figure shows that the first few meters of rock above the drift are being heated faster than is rock at the equivalent distance below the drift. This plot shows a smooth increase in temperature with time for this profile and also indicates that, at a distance of $10 \mathrm{~m}$ from the HD axis, rock was still at approximately ambient temperature on February 28. 
Figure 15 shows temperatures, on February 28, for the vertical upward and downward boreholes in this cross section as well as for the boreholes at $\pm 45^{\circ}$ and $\pm 135^{\circ}$. Temperature is plotted as a function of vertical distance from the HD axis.

Boreholes above the HD axis include borehole 158 oriented at $0^{\circ}$ (vertical upward) and boreholes 159 and 165 oriented at $-45^{\circ}$ and $+45^{\circ}$ respectively (see Table 1 ). Data for these boreholes indicates the following: First, the temperature field above the HD in this cross section is very symmetric about a vertical plane through the axis of the HD. Temperature is highest directly above the HD, and temperature distributions vs. distance above the HD axis are identical for the two sides of the HD and are less than $2{ }^{\circ} \mathrm{C}$ lower than are the temperatures directly above the drift. This is similar to temperatures shown in Figure 11 and indicates that regions above the wing heaters are being heated evenly and at the same rate as is the rock above the HD. This symmetric temperature distribution indicates that, as of February 28, the DST is successfully progressing toward the goal of developing a horizontally planar heated zone above the HD.

Boreholes below the HD axis include borehole 162 oriented at $180^{\circ}$ (vertical downward) and boreholes 161 and 163 oriented at $-135^{\circ}$ and $+135^{\circ}$ respectively (see Table 1 ). Data for these boreholes show a very even temperature distribution in the downward direction because temperature at a given distance below the plane of the $\mathrm{HD}$ axis varies less than $2^{\circ} \mathrm{C}$ in any of the boreholes. Data for these boreholes are consistent with data shown in Figure 11 and indicate that temperatures below the plane of the heaters are lower than those at the same distance above the heaters. This indicates the importance of both convection and conduction as heat-transfer mechanisms.

\subsubsection{Horizontal Boreholes}

Figure 16 depicts temperature history for the horizontal boreholes in this cross section (boreholes 160 and 164) and shows several interesting features of the temperature in the horizontal plane.

- As seen in Figure 12, the temperature field is approximately symmetric, although not as symmetric as observed in the inclined boreholes.

- At distances $<14 \mathrm{~m}$ from the drift, temperature variations as great as $\pm 20^{\circ} \mathrm{C}$ occur along these boreholes. These temperature variations are correlated with the segments of the wing heaters. Lower temperatures are associated with the zone between HD and inner segment of wing heater, and the zone between inner and outer wing heater segments.

- Temperatures are more irregular on the south (-x or AOD) side (borehole 164$)$, primarily due to higher temperatures associated with the outer wing heaters at this location.

- Level temperatures of $96^{\circ} \mathrm{C}$ indicate boiling occurred in significant zones. In particular, boiling is indicated for February 20 at depths between 10 and $12 \mathrm{~m}$ in borehole 164; this persists through February 28. No boiling zone is apparent on the North $(+x)$ side of the HD.

- No evidence of boiling is found near the drift wall. This situation is similar to the temperature distribution shown in the vertical and boreholes and those at $\pm 45^{\circ}$ and may indicate that drying has occurred in the first meter or so of rock around the drift wall.

- At distances beyond $14 \mathrm{~m}$ from the drift axis (the end of the wing heaters), temperature declines rapidly back to ambient levels. 
Comparison with Figure 12 indicates that, in this cross section, the heated region is slightly wider than that observed in the section at $12 \mathrm{~m}$ from the bulkhead. In this cross section, temperatures at $16 \mathrm{~m}$ from the bulkhead are approximately $45^{\circ} \mathrm{C}$, compared to $40^{\circ} \mathrm{C}$ at the equivalent location in the 12-m cross section.

Figure 17 shows data from boreholes 160 and 164 as a surface similar to that shown in Figure 13. In Figure 17, the vertical axis represents temperature, and horizontal distance from the center of the drift is shown across the page. Time from the start of the test in days is shown increasing from the front to the back of the plot. This surface represents a linear interpolation of data presented in Figure 16. Temperatures shown within the drift are an artifact of the surface rendering and are not representative.

This plot illustrates the symmetry of the temperature field over time and the persistence of the low temperatures in the zones between heaters. The leveling, with time, of the temperature at approximately $96^{\circ} \mathrm{C}$ (indicating boiling) is effectively illustrated for borehole 164. Temperature gradients are highest at the sides of the wing heaters.

\subsection{Cross Section at $39 \mathrm{~m}$}

\subsubsection{Vertical and Inclined Boreholes}

Figure 18 depicts temperature in boreholes 170 and 175 at a series of times. These boreholes are vertical upward and downward, respectively, as shown on the inset. Temperature is plotted as a function of vertical distance from the axis of the HD. This figure shows that the first few meters of rock above the drift is being heated faster that is rock at the equivalent distance below the drift. This plot depicts a smooth increase in temperature with time for this profile and also shows that, at a distance of $10 \mathrm{~m}$ from the HD axis, rock was still at approximately ambient temperature on February 28.

Figure 19 shows temperatures, on February 28, for the vertical upward and downward boreholes in this cross section as well as for the boreholes at $\pm 45^{\circ}$ and $\pm 135^{\circ}$. Temperature is plotted as a function of vertical distance from the HD axis.

Boreholes above the HD axis include borehole 170 oriented at $0^{\circ}$ (vertical upward) and boreholes 171 and 175 oriented at $-45^{\circ}$ and $+45^{\circ}$, respectively (see Table 1 ). Data for these boreholes indicates the following: First, the temperature field above the HD in this cross section is very symmetric about a vertical plane through the axis of the HD. Temperature is highest directly above the HD, and temperature distributions vs. distance above the HD axis are identical for the two sides of the HD and are less than $2^{\circ} \mathrm{C}$ lower than is the temperatures directly above the drift. This indicates that regions above the wing heaters are being heated evenly and at the same rate as is the rock above the HD. This symmetric temperature distribution indicates that, as of February 28, the DST is successfully progressing toward the goal of developing a horizontally planar heated zone above the HD.

Boreholes below the HD axis include borehole 173 oriented at $180^{\circ}$ (vertical downward) and boreholes 172 and 174 oriented at $-135^{\circ}$ and $+135^{\circ}$, respectively (see Table 1 ). Data for these boreholes show a very even temperature distribution in the downward direction because temperature at a given distance below the plane of the HD axis varies less than $1^{\circ} \mathrm{C}$ in any of the boreholes. Data for these boreholes are consistent with data shown in Figure 7 and indicate that temperatures below the plane of the heaters are lower than those at the same distance above the heaters. This indicates the importance of both convection and conduction as heat-transfer mechanisms. 


\section{Relative Humidity and Barometric Pressure}

Relative humidity (RH) is an important parameter for assessment the performance of waste packages and the overall repository. In particular, drift $\mathrm{RH}$ above $85 \%$ is considered undesirable from the point of view of waste package corrosion. Humidity sensors and pressure transducers have been placed about $1 \mathrm{~m}$ inside the bulkhead in the HD. The humidity sensors are considered to provide only qualitative information on humidity at temperatures greater than $50^{\circ} \mathrm{C}$ because calibration facilities at temperatures $>50^{\circ} \mathrm{C}$ were unavailable.

RH data for the two RH sensors, along with air (barometric) pressure, are plotted in Figure 20. This plot shows that, upon the start of heating, humidity values dropped from approximately $30 \%$ to $15 \%$. During the reporting period, $\mathrm{RH}$ varied between approximately $10 \%$ and $25 \%$. It is interesting to note that the $\mathrm{RH}$ data are inversely correlated with the air pressure in the drift.

After approximately 40 days of heating, condensation was observed on various surfaces near the bulkhead, and a puddle formed on the concrete floor in front of the bulkhead (see Figure 21). These wet conditions near the bulkhead alternated with drier conditions. Moreover, the wetter periods coincided with high RH in the HD, and the dry conditions near the bulkhead coincided with low RH inside the HD.

During the barometric pressure highs, the gas in the heater drift is likely to be largely static; thus, mass transport during this time is slow and probably dominated by the binary diffusion of air into the HD through the bulkhead. This leads to a low RH. During periods of changing barometic pressure, air flow will be out of the drift, increasing the influx of moist air into the drift from the drift walls and increasing the RH. Further analysis of this behavior will provide insight into moisture transport associated with in-drift heating and will provide information valuable for assessment of the performance of a potential repository.

\section{Evaluation of Sensors}

This section presents an evaluation of RTD performance through the first quarter of heating in the DST. Also presented are corrections to sensor locations for borehole 134 and suggested changes to data-channel assignments of a portion of the RTDs in boreholes 79 and 80.

\subsection{Listing of Failed and Suspect Sensors}

Approximately1860 RTD sensors were emplaced in the 28 boreholes used to monitor temperature. Thirty of these sensors had failed or were giving readings considered unreliable as of February 28, 1998. These are listed in Table 2. This constitutes a failure rate of approximately $1.6 \%$. The performance of these sensors will continue to be monitored, and the reliability evaluated in future reports. 
First Quarter Report of Temperature Measurements

During the Heating Phase of the Drift-Scale Test

Table 2 Listing of RTD sensors suspected of malfunction

\begin{tabular}{|c|c|c|c|}
\hline Borehole Name & RTD\# & Start of Malfunction & Problem-Cause \\
\hline ESF-HD-80-TEMP-2 & 46 & $12 / 3 / 97$ & Bad-shorted \\
\hline ESF-HD-133-TEMP-3 & 64 & $12 / 3 / 97$ & Bad-shorted \\
\hline ESF-HD-138-TEMP-6 & 32 & $12 / 3 / 97$ & Bad-shorted \\
\hline ESF-HD-138-TEMP-6 & 40 & $12 / 10 / 97$ & \\
\hline ESF-HD-138-TEMP-6 & 47 & 2/28/98 & Over-range \\
\hline ESF-HD-139-TEMP-7 & 31 & $2 / 10 / 98$ & Over-range \\
\hline ESF-HD-141-TEMP-9 & 36 & $12 / 3 / 97$ & Over-range \\
\hline ESF-HD-141-TEMP-9 & 40 & $2 / 28 / 98$ & Over-range \\
\hline ESF-HD-141-TEMP-9 & 41 & $1 / 30 / 98$ & Low \\
\hline ESF-HD-143-TEMP-11 & 24 & $2 / 28 / 98$ & Low \\
\hline ESF-HD-143-TEMP-11 & 40 & $12 / 3 / 97$ & Over-range \\
\hline ESF-HD-143-TEMP-11 & 42 & 2/28/98 & Low \\
\hline ESF-HD-143-TEMP-11 & 67 & $12 / 3 / 97$ & Over-range \\
\hline ESF-HD-144-TEMP-12 & 33 & & \\
\hline ESF-HD-158-TEMP-13 & 7 & $12 / 3 / 97$ & Bad-shorted \\
\hline ESF-HD-161-TEMP-16 & 64 & $12 / 3 / 97$ & Low \\
\hline ESF-HD-168-TEMP-21 & 28 & $12 / 3 / 97$ & Low \\
\hline ESF-HD-168-TEMP-21 & 31 & & Bad-shorted \\
\hline ESF-HD-168-TEMP-21 & 40 & $12 / 3 / 97$ & Low \\
\hline ESF-HD=169-TEMP-22 & 5 & $1 / 30 / 98$ & Over-range \\
\hline ESF-HD-169-TEMP-22 & 22 & $12 / 3 / 97$ & Low \\
\hline ESF-HD-169-TEMP-22 & 29 & $12 / 3 / 97$ & Low \\
\hline ESF-HD-169-TEMP-22 & 38 & $12 / 3 / 97$ & Low \\
\hline ESF-HD-169-TEMP-22 & 2 & $12 / 3 / 97$ & Low \\
\hline ESF-HD-170-TEMP-23 & 28 & $12 / 3 / 97$ & Low \\
\hline ESF-HD-171-TEMP-24 & 53 & $12 / 3 / 97$ & Over-range \\
\hline ESF-HD-172-TEMP-25 & 2 & $2 / 10 / 98$ & Over-range \\
\hline ESF-HD-173-TEMP-26 & 42 & $12 / 3 / 97$ & Low \\
\hline ESF-HD-175-TEMP-28 & 31 & $12 / 3 / 97$ & Low \\
\hline ESF-HD-175-TEMP-28 & 34 & $12 / 3 / 97$ & Bad-shorted \\
\hline
\end{tabular}

\subsection{Suggested Corrections for RTD Data}

Borehole 79-This borehole was designed to be parallel to the HD. Survey data of the borehole show that it is actually dipping downward, and survey points are not available beyond $+10 \mathrm{~m}$ past the bulkhead. The increase in temperature with depth in this borehole is 
consistent with the borehole dipping toward the plane of the wing heaters. The exact locations of the thermocouples in this borehole are not well known, and the data cannot be properly interpreted at this writing.

The see-saw nature of the data reported for sensors RTD-25 through RTD-58 in this borehole is unphysical and suggests that, for these sensors, pairs of data values may be switched. Thus, the value reported for RTDs 25 and 26 should be switched. A similar situation exists for RTDs 26 and 28 up through RTDs 57 and 58.

Borehole 80-Data collection system (DCS) temperature data for the first 24 RTDs in borehole 80 exhibit an unphysical see-saw pattern that suggests that pairs of sensors may be switched (i.e., the location of the RTDs is correct, but the value reported for pairs of RTDs may be switched). Thus, the value reported for RTDs 1 and 2 should be switched. A similar situation exists for RTDs 3 and 4 up through RTDs 23 and 24. Data plotted in Figures 8 and 9 of this report have been edited to reflect such a change. Appendix B presents suggested revised assignments for the first 24 RTDs in borehole 80.

Borehole 134-Original data for this borehole indicated that temperatures were lower at this location than for the other four vertical downward boreholes. Examination of the notebook documenting installation indicates that the coordinates submitted in the XYZ documentation (Lin and Wagoner, 1998) are in error. Revised locations of RTD sensors in this borehole are presented Appendix C.

Borehole 160-Temperature sensors RTD-27 and RTD-29 (located between 10 and $11 \mathrm{~m}$ depth in this borehole) do not follow the overall trend of the thermal profile of the borehole. It is possible that these sensors are reversed.

\section{Analysis}

Temperature data reported here indicate that, at the end of the first quarter of heating (February 28, 1998), rock near the top of the drift had reached a temperature of approximately $105 \pm 5^{\circ} \mathrm{C}$, while temperatures near the bottom of the drift (in the invert) were approximately $10^{\circ} \mathrm{C}$ cooler. Temperature data also indicate that temperature variations along the length of the drift between 3 and $39 \mathrm{~m}$ from the bulkhead were small.

Moreover, temperatures measured in cross sections perpendicular to the drift axis indicate that the temperature field is very symmetric about a vertical plane through the axis of the HD. Temperature is highest directly above the HD; temperature distributions vs. distance above the HD axis are identical for the two sides of the HD and are less than $2^{\circ} \mathrm{C}$ lower than the temperatures directly above the drift. This indicates that regions above the wing heaters are being heated evenly and at the same rate as is the rock above the HD. This symmetric temperature distribution indicates that, as of February 28, the DST is successfully progressing toward the goal of developing a horizontally planar heated zone above the HD.

Temperatures below the plane of the heaters are lower than those at the same distance above the heaters. This indicates the importance of both convection and conduction as heattransfer mechanisms. The difference in temperature between rock at the top and bottom of the drift is probably caused by convection in the drift. This is useful data for guiding the numerical modeling of the test because it reflects the relative strength of air convection and radiation as heat-transfer mechanisms. 
Temperatures in horizontal boreholes show a temperature field that is approximately symmetric, although not as symmetric as observed in the inclined boreholes. Temperature variations along horizontal boreholes are correlated with the segments of the wing heaters. Lower temperatures are associated with zone between HD and inner segment of wing heater and with the zone between inner and outer wing heater segments. Temperature appears to have been higher on the south (AOD) side of the HD, especially in zones influenced by the outer wing heaters. Level temperatures at $96^{\circ} \mathrm{C}$ in mid to late February indicate boiling was occurring in significant zones. These zones begin approximately $5 \mathrm{~m}$ from the drift wall. No evidence of boiling is found near the drift wall.

A preliminary comparison of the measured vertical temperature profile at $12 \mathrm{~m}$ from the bulkhead and a simulation of temperature at that location using the NUFT code is shown in Figure 22, which shows good agreement between the measured and predicted temperatures. This was expected during the early portion of the test when conduction is thought to have dominated the thermal transport. The correlation between $\mathrm{RH}$ and barometric pressure is indicative of barometric pumping of moisture mobilized by heating of the DST.

\section{References}

Birkhozer, J.T., and Y.W. Tsang (1997). "Pretest Analysis of the Thermal-Hydrological Conditions of the ESF Drift Scale Test." Milestone report for the CRWMS Management and Operating Contractor, U.S. Department of Energy. (SP9322M4) Berkeley, CA: Lawrence Berkeley National Laboratory. [MOL.19971201.0805]

Buscheck, T.A., and J.J. Nitao (1995). “Thermal-Hydrological Analysis of Large-Scale Thermal Tests in the Exploratory Studies Facility at Yucca Mountain." (UCRL-ID121791) Livermore, CA: Lawrence Livermore National Laboratory. [MOL.19960501.0392; 223657]

Buscheck, T.A., R.J. Shaffer, and J.J. Nitao (1997). "Pretest Thermal-Hydrological Analysis of the Drift-Scale Thermal Test at Yucca Mountain." (SP9318M4) Livermore, CA: Lawrence Livermore National Laboratory.

CRWMS M\&O (1996). "Test Design, Plans, and Layout for the First ESF Thermal Test." (BAB000000-01717-4600-00025, Rev. 1) Las Vegas, NV: Civilian Radioactive Waste Management System Management and Operation Contractor: TRW Environmental Safety Systems, Inc. [MOL.19970114.0166]

Lin, W., and J. Wagoner (1998). "DST As-Built TDIF, XYZ Coordinates of the Sensors in the Drift-Scale Test." Milestone report for the CRWMS Management and Operating Contractor, U.S. Department of Energy. (SPY195M4) Livermore, CA: Lawrence Livermore National Laboratory.

SNL (1998). "Evaluation and Comparative Analysis of the Drift-Scale Test Thermal and Thermomechanical Data: First Quarter Results." Milestone report for the CRWMS Management and Operating Contractor, U.S. Department of Energy. (SP2520M4) Albuquerque, NM: Sandia National Laboratories. 
Figures 



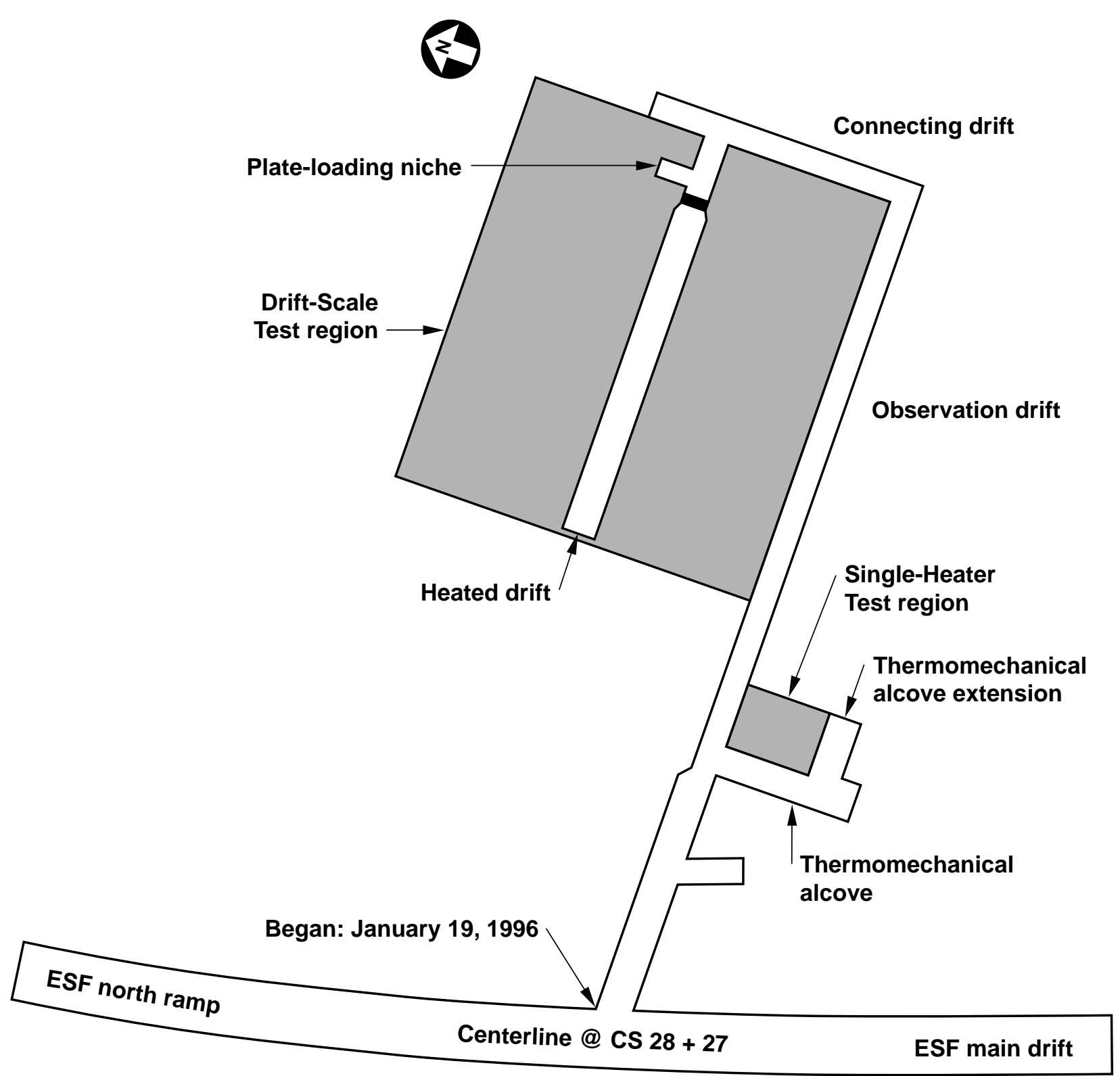

Reference only (Scale approximate)

Figure 1. Configuration of the Drift-Scale Test. 


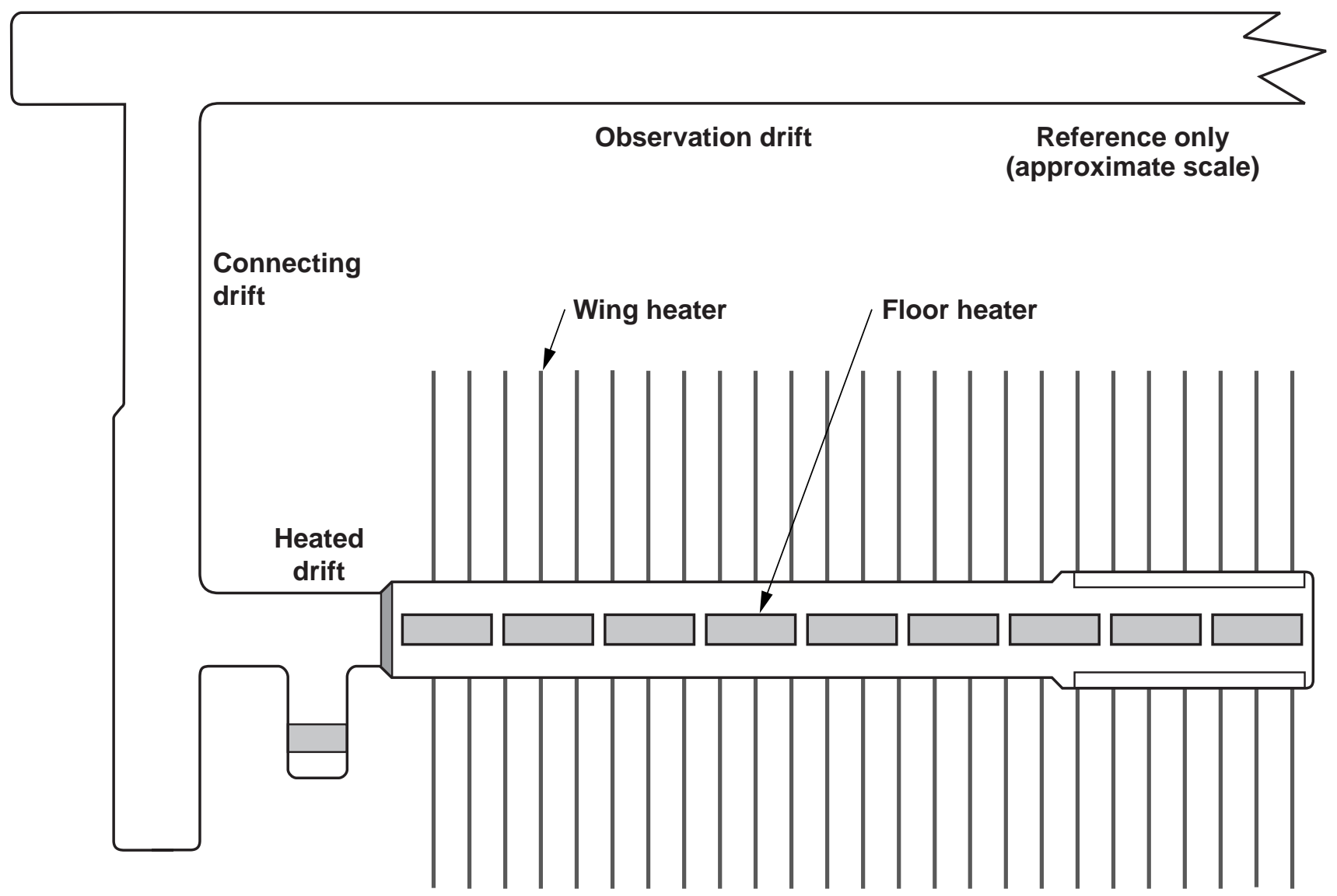

Figure 2. Plan view showing locations of floor and wing heaters. 


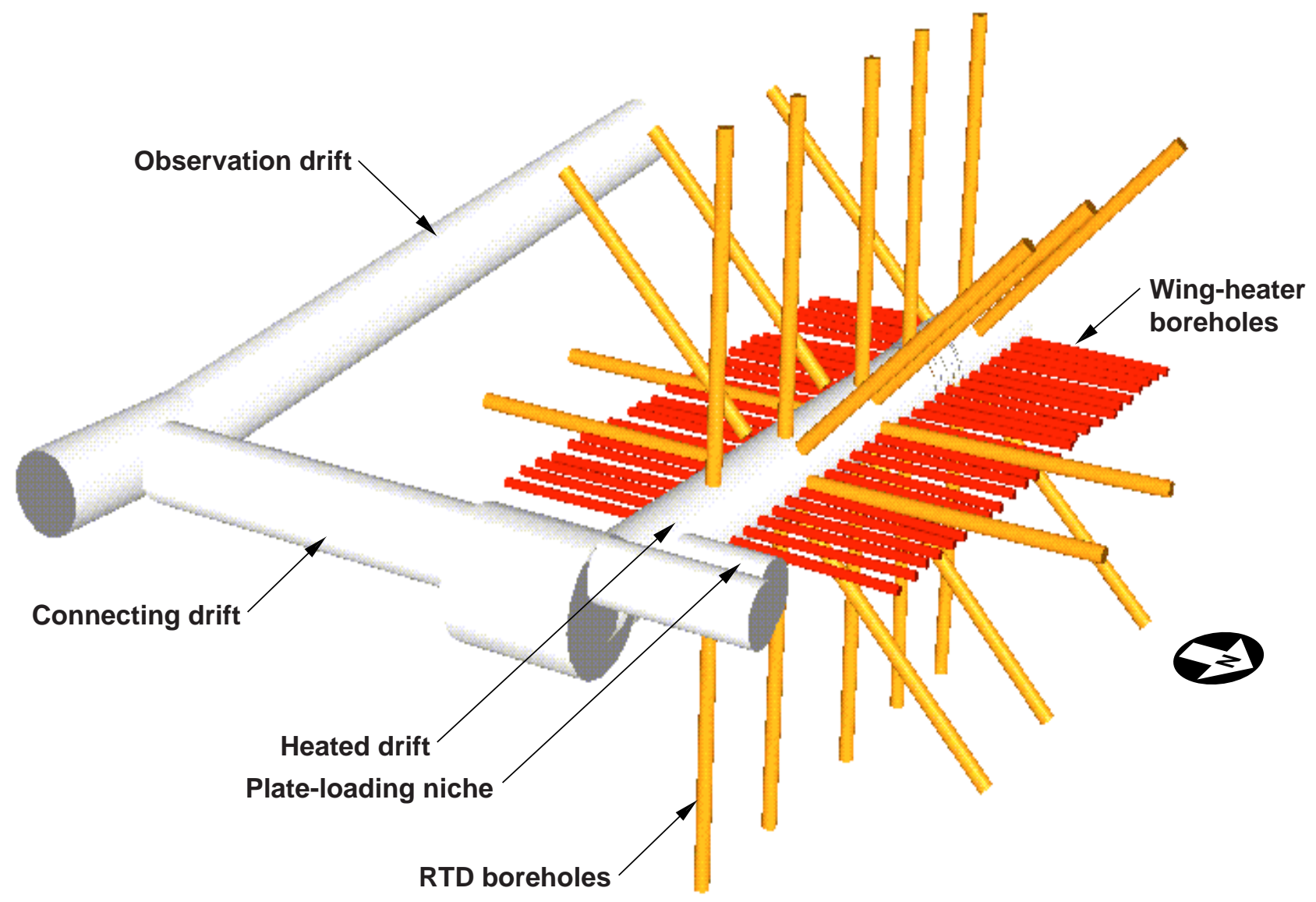

Figure 3. Drift-Scale Test drift configuration showing borehole locations. 


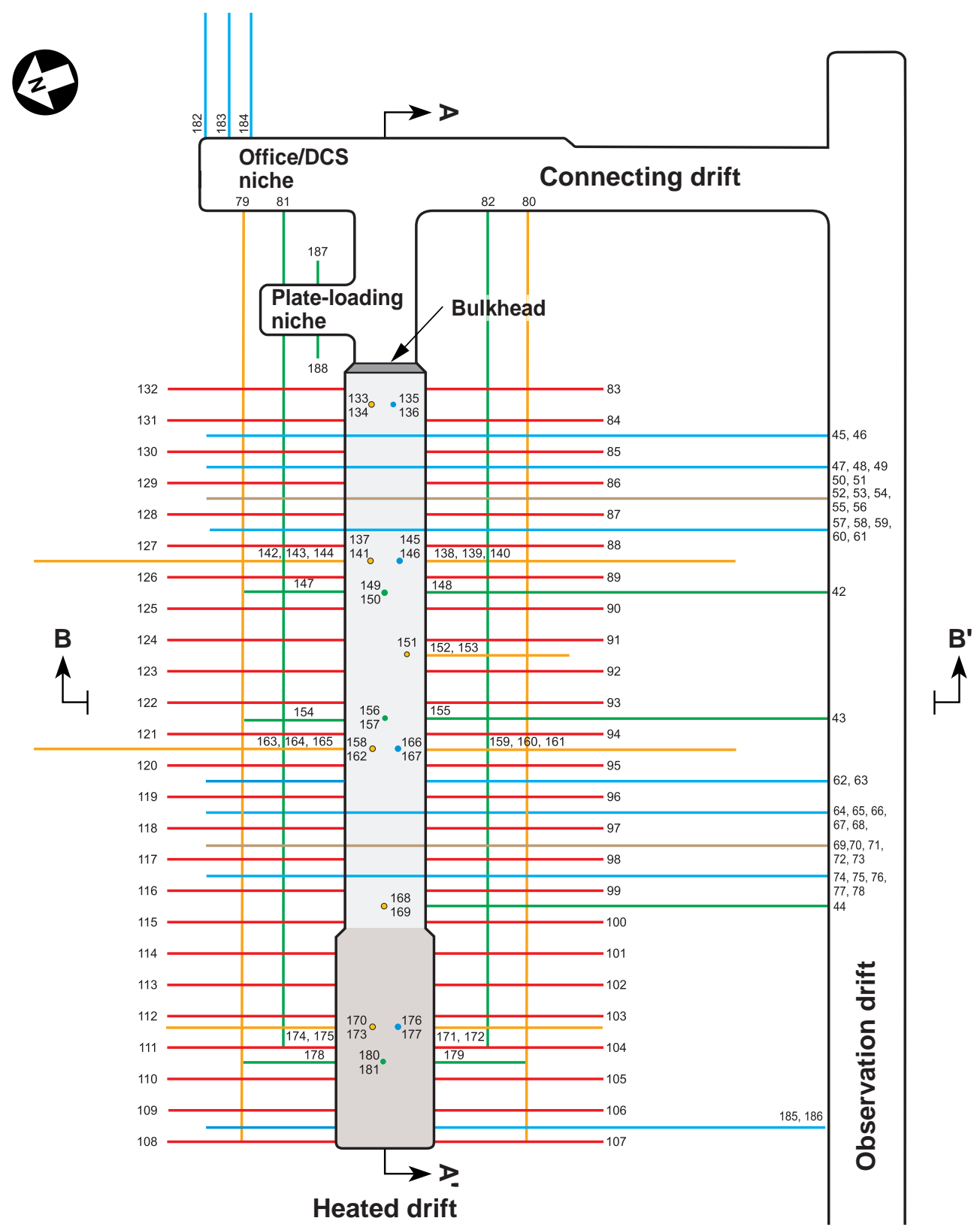

\section{LEGEND: Boreholes}

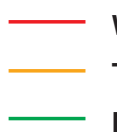

$\begin{array}{lll}\text { Wing Heaters } & \text { Hydrological } \\ \text { Thermal } & \text { Chemical } \\ \text { Mechanical } & \end{array}$

REFERENCE ONLY

(approximate scale: $1 \mathrm{~cm} \sim 5 \mathrm{~m}$ )

\section{LEGEND: Ground Support System}

Rockbolts and welded wire used throughout heated drift for safety and stability purposes only

Cast-in-place concrete

NOTE: See Table 1 for detailed description of numbered boreholes.

Figure 4a. Relative geometry of boreholes in cross sections 12, 23, and $39 \mathrm{~m}$ from the bulkhead (top view). 


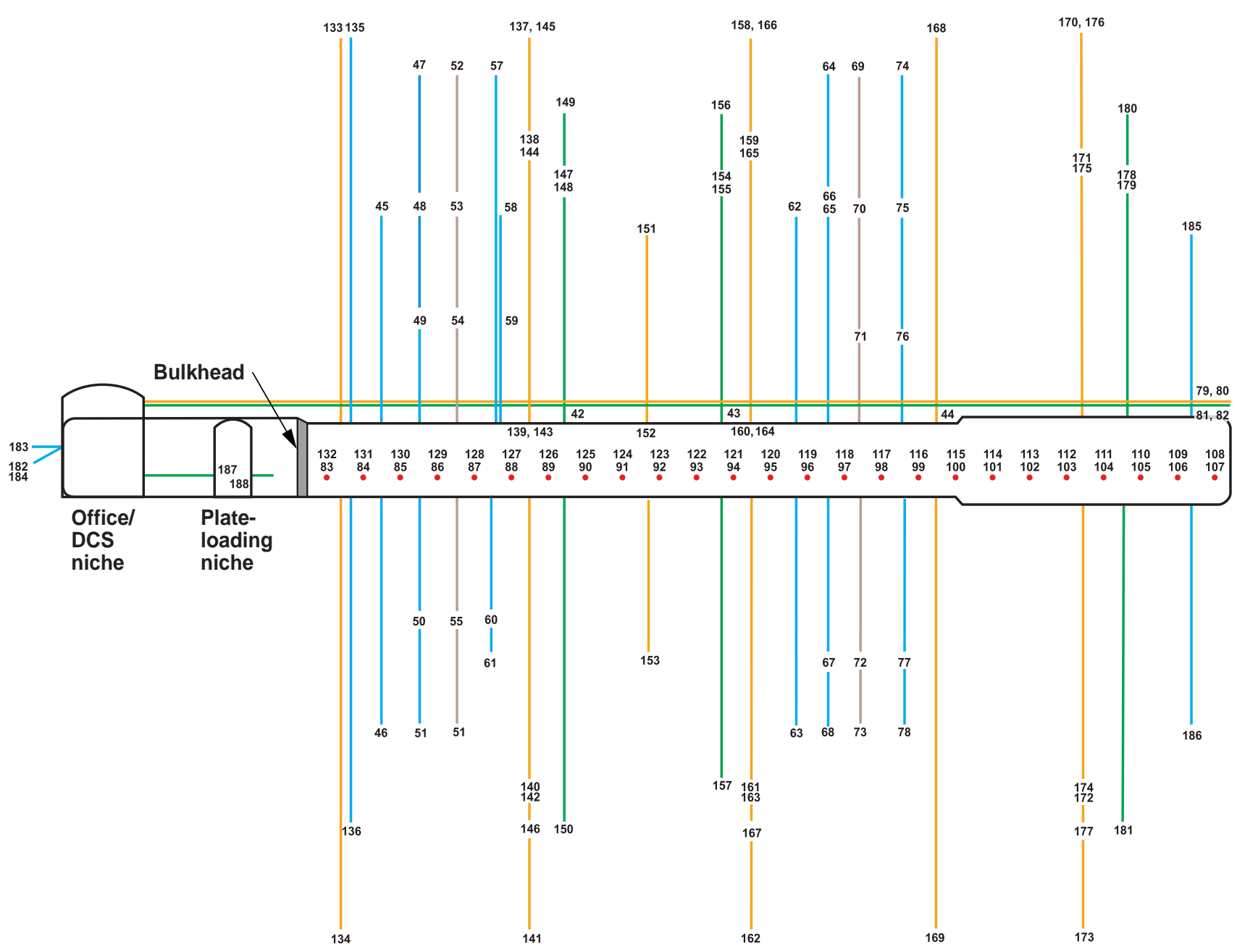

\section{LEGEND: Boreholes}

$\begin{array}{ll}- & \text { Wing Heaters } \\ -\quad \text { Thermal } \\ -\quad \text { Mechanical } \\ -\quad \text { Hydrological } \\ \quad \text { Chemical }\end{array}$

REFERENCE ONLY

(approximate scale: $1 \mathrm{~cm} 3 \mathrm{~m}$ )

Figure $4 \mathrm{~b}$. Relative geometry of boreholes in cross sections 12, 23, and $39 \mathrm{~m}$ from the bulkhead (side view of cross section $\mathrm{A}-\mathrm{A}^{\prime}$ in Figure 4a). 


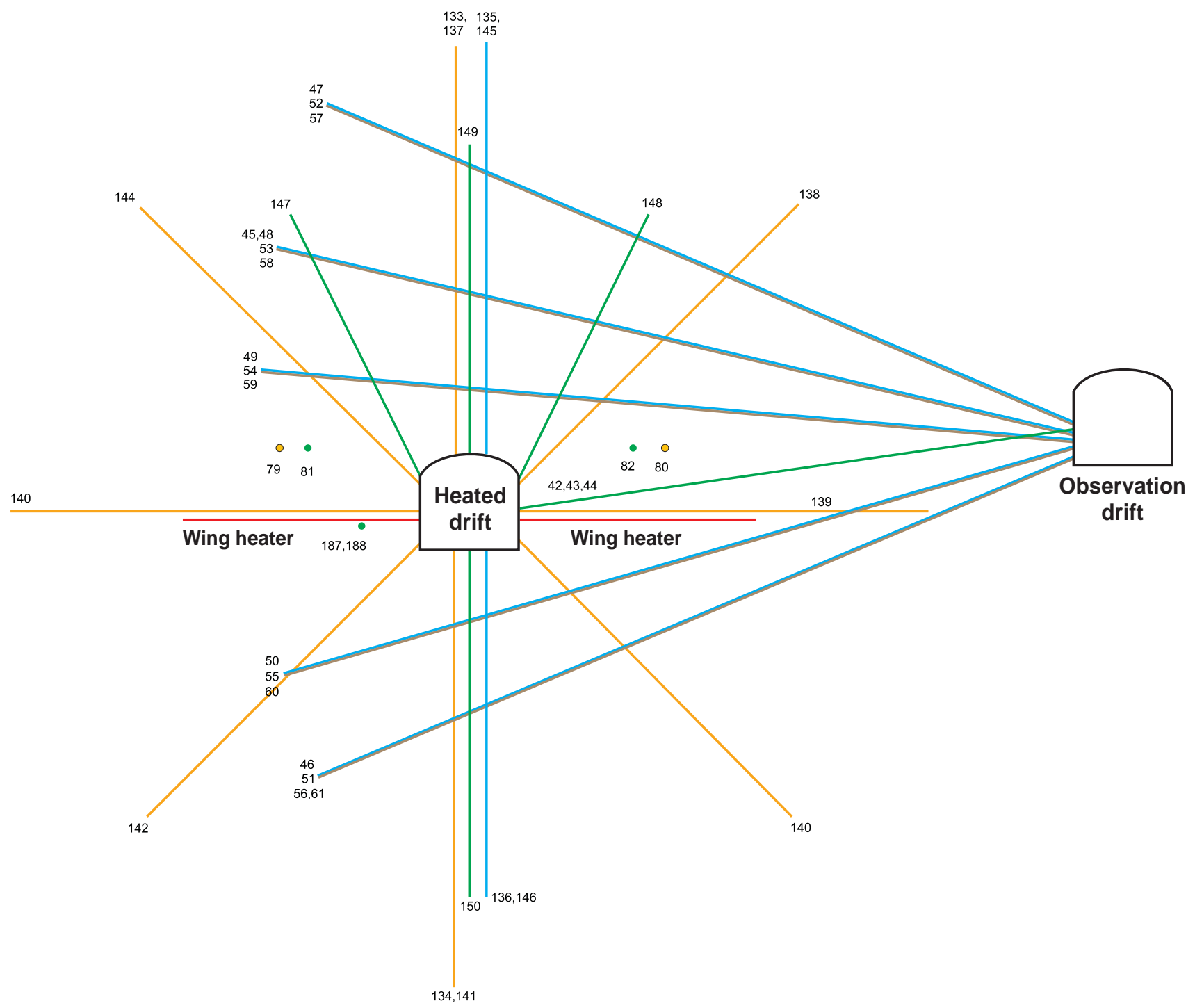

LEGEND: Boreholes

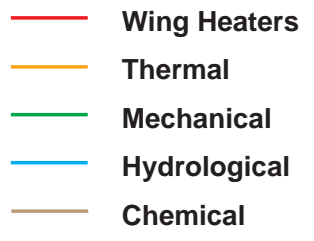

REFERENCE ONLY

(approximate scale: $1 \mathrm{~cm} \sim 3 \mathrm{~m}$ )

Figure 4c. Relative geometry of boreholes in cross sections 12, 23, and $39 \mathrm{~m}$ from the bulkhead end view of cross section B-B' in Figure 4a). 


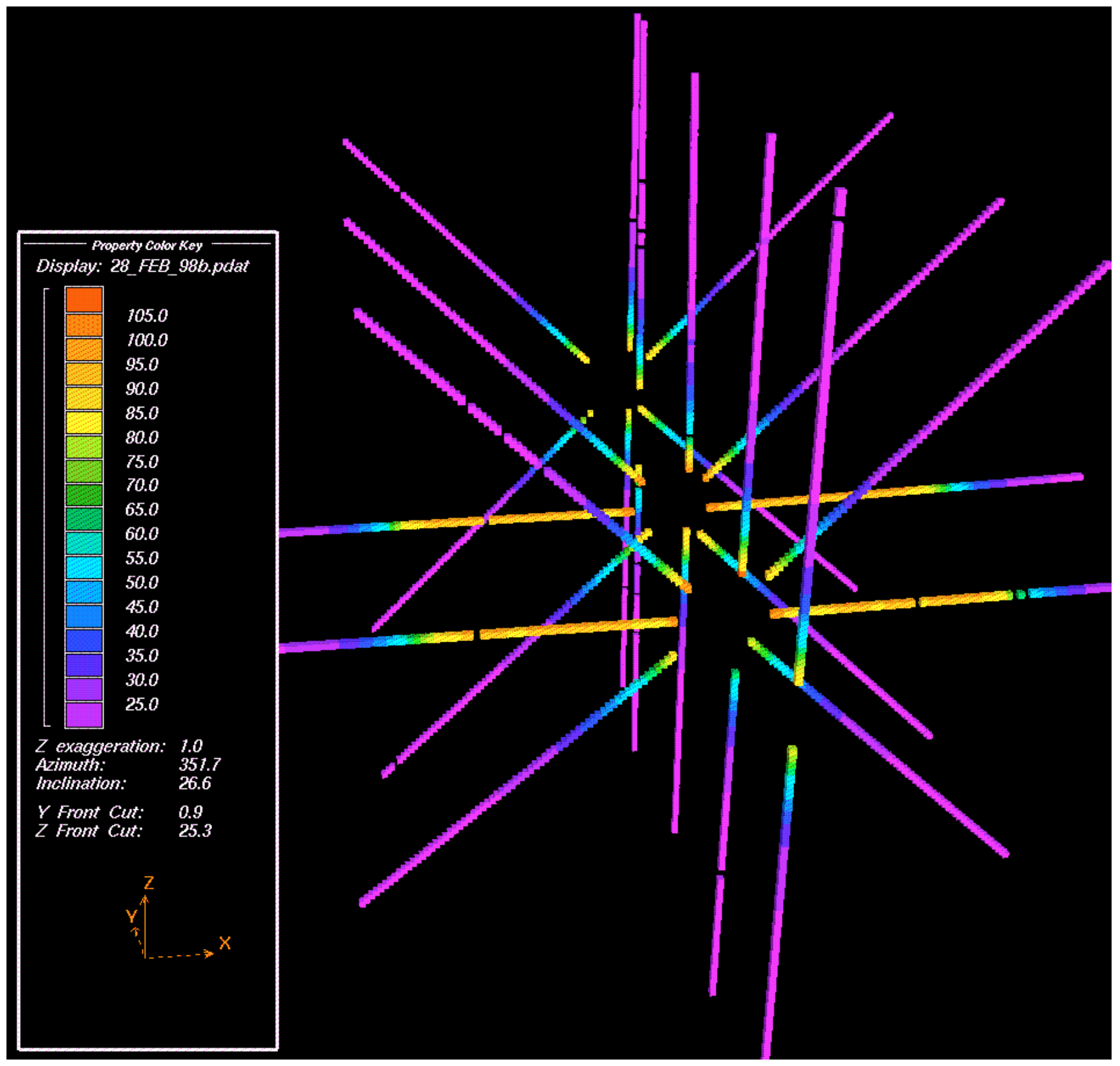

Figure 5. Drift-Scale Test temperature for radial RTD boreholes on February 28, 1998. 


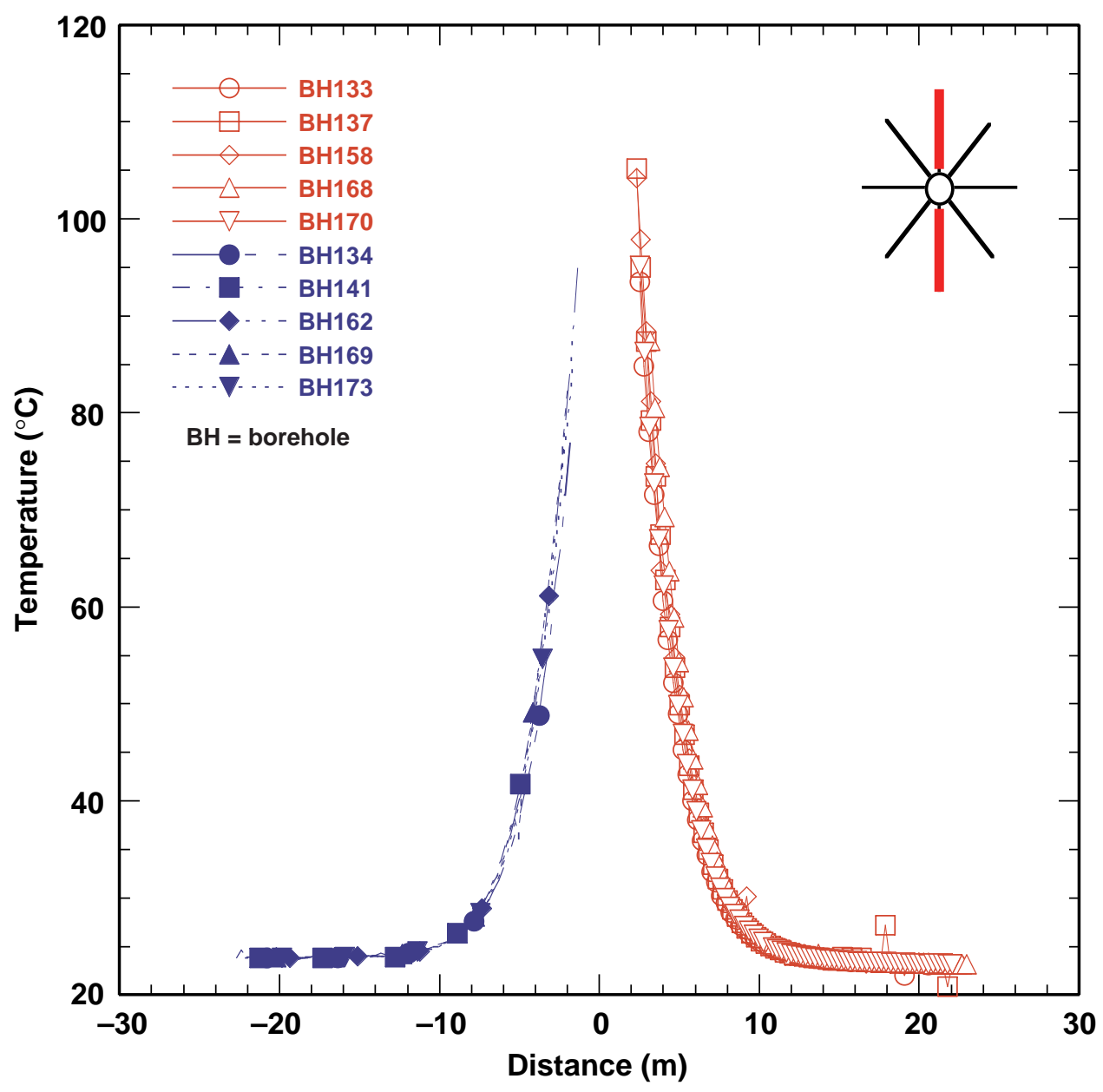

Figure 6. Temperature for vertical holes on February 28, 1998. 


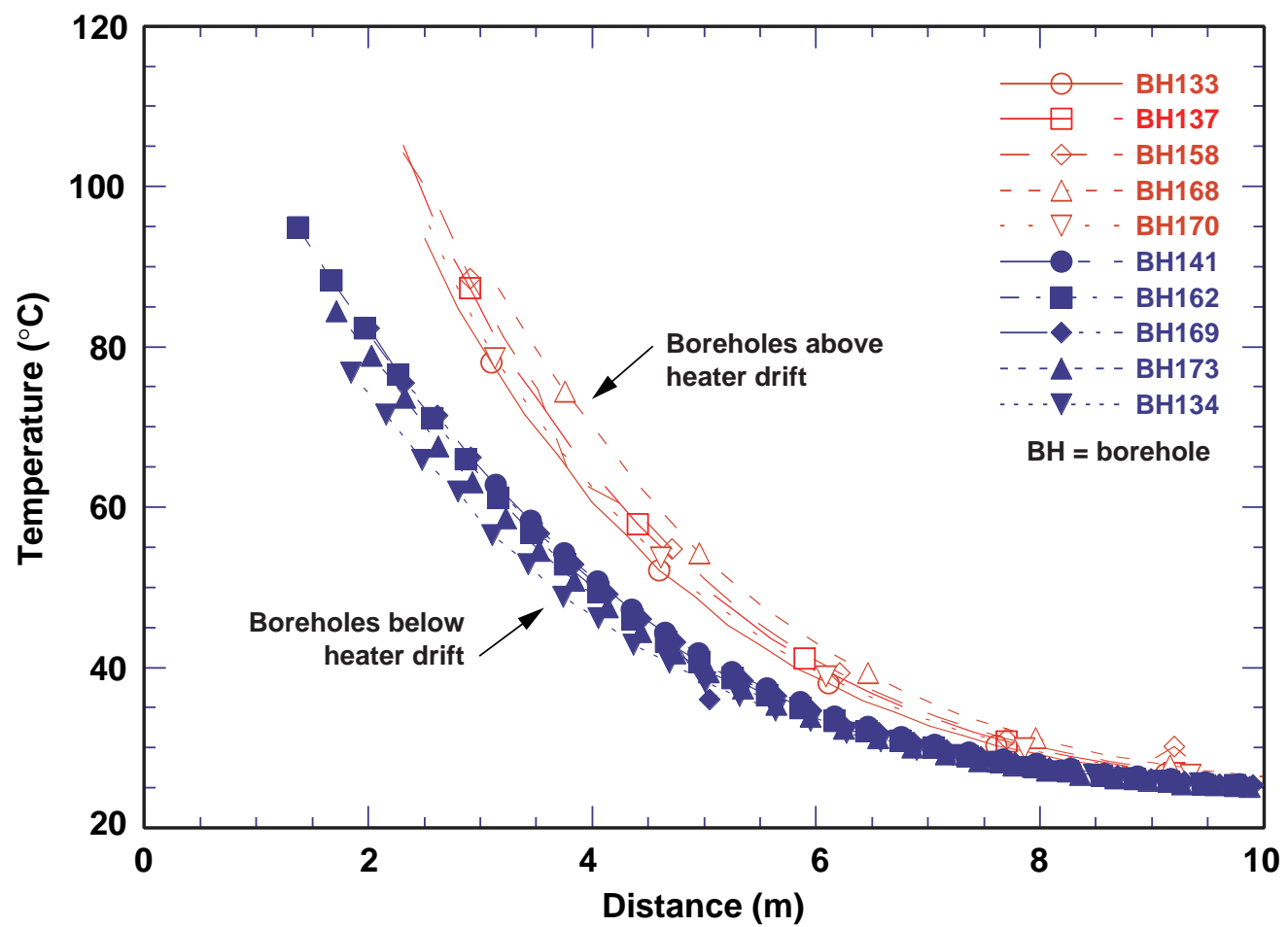

Figure 7. Temperature plotted as a function of distance from drift axis, in same 10 boreholes shown in Figure 6. 


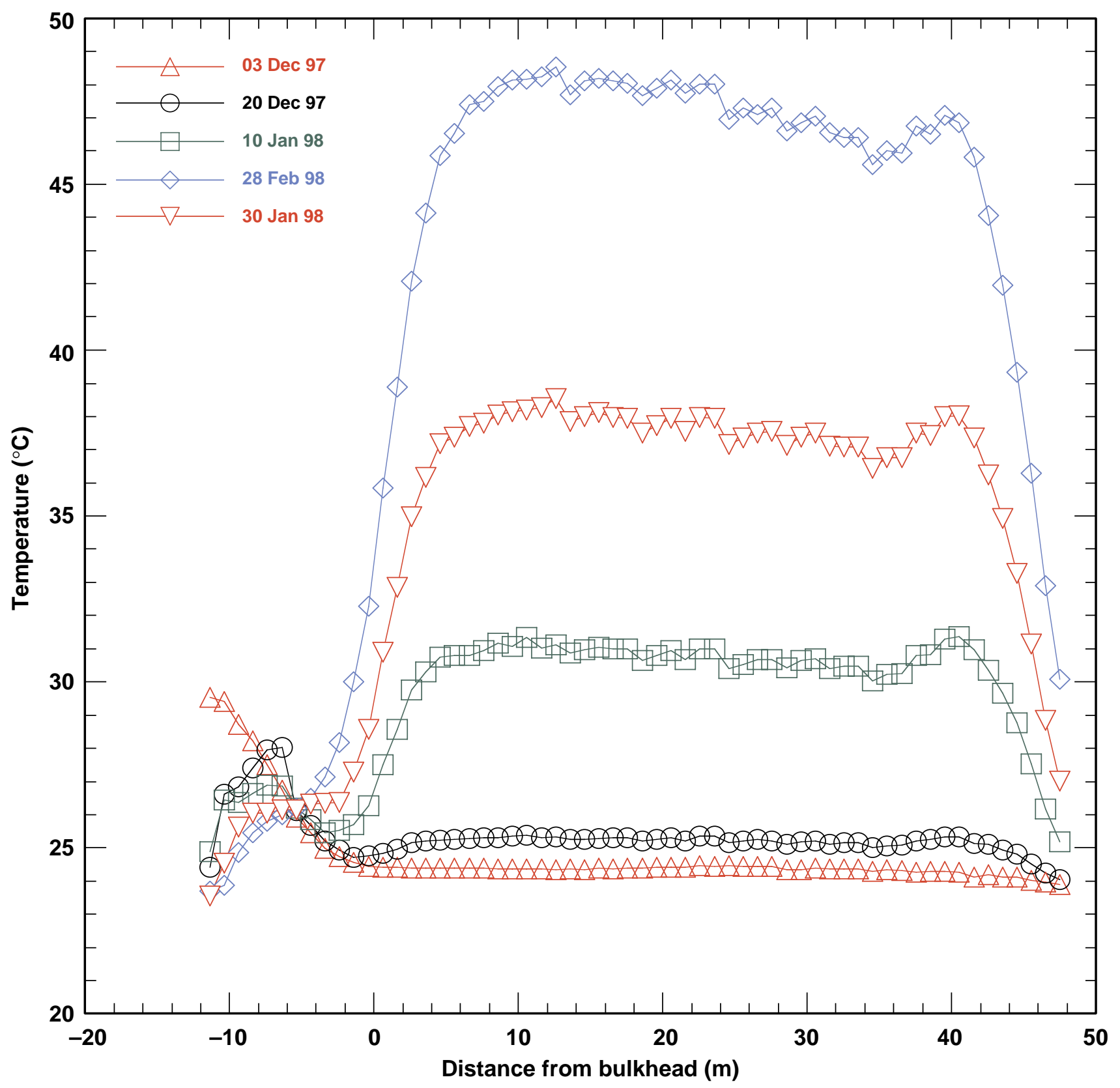

Figure 8. Temperature, as a function of distance form the bulkhead, for borehole 80 . 


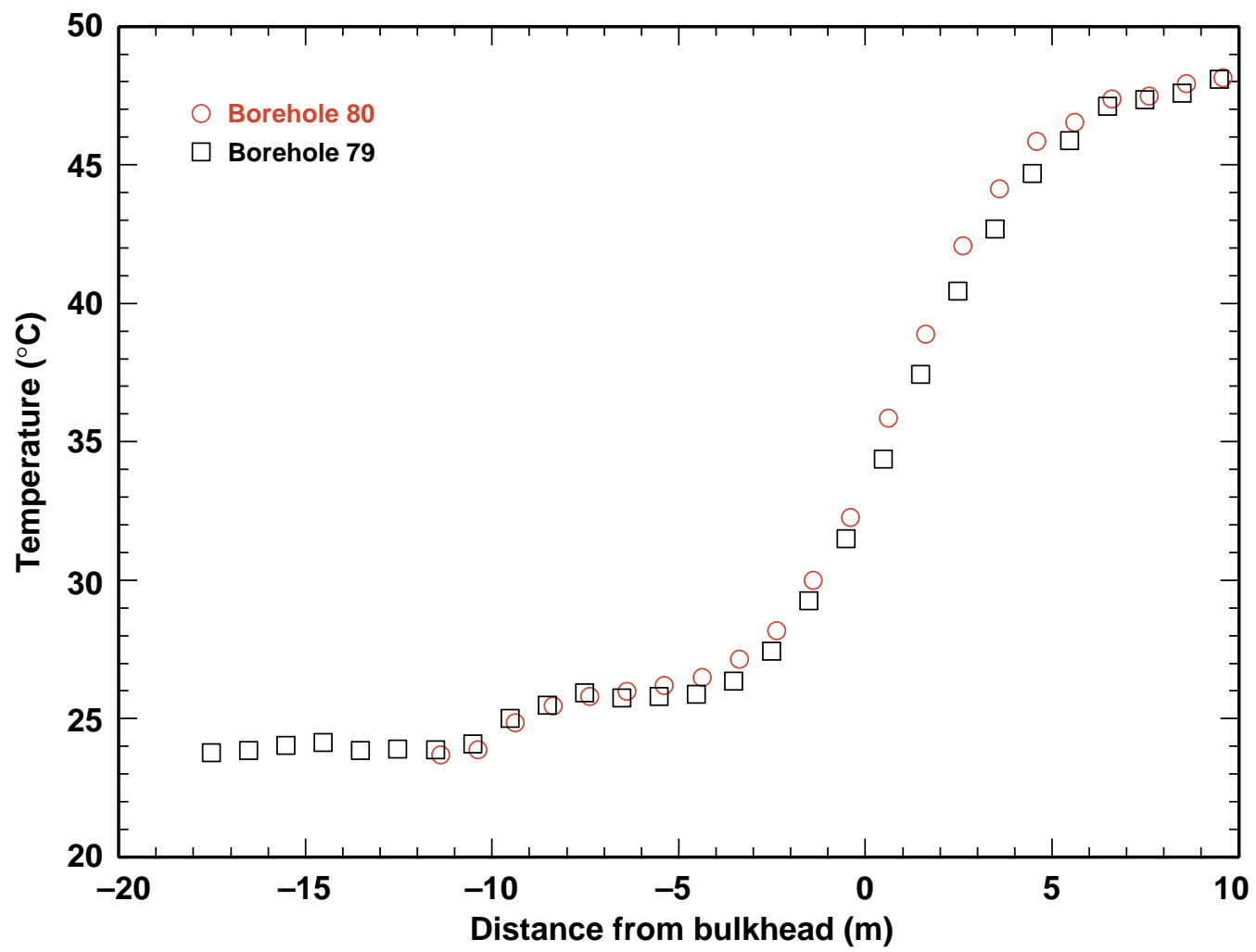

Figure 9. Temperatures on February 28, 1998, for boreholes 79 and 80. 


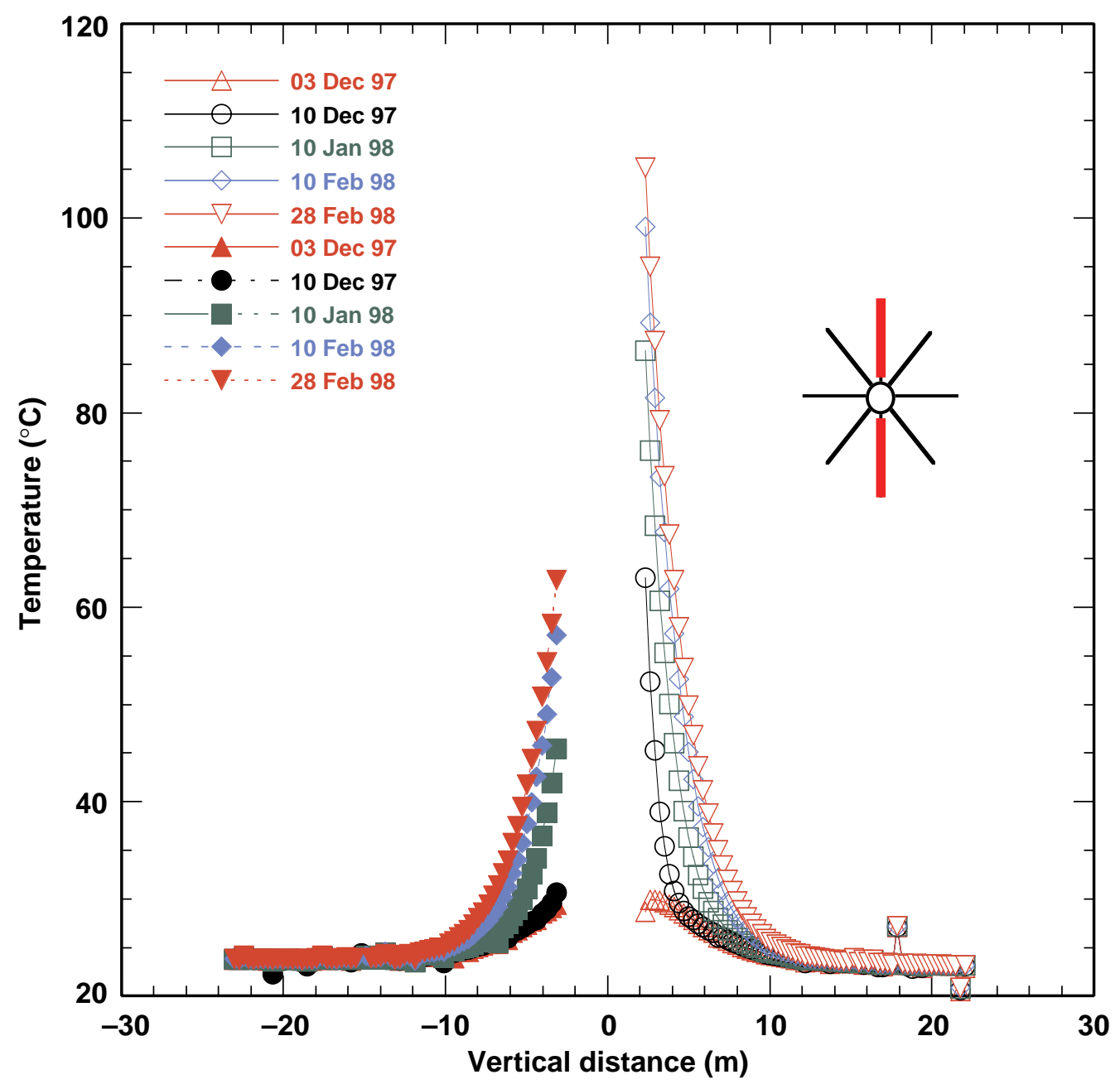

Figure 10. Temperature in boreholes 137 and 141 for various dates. 


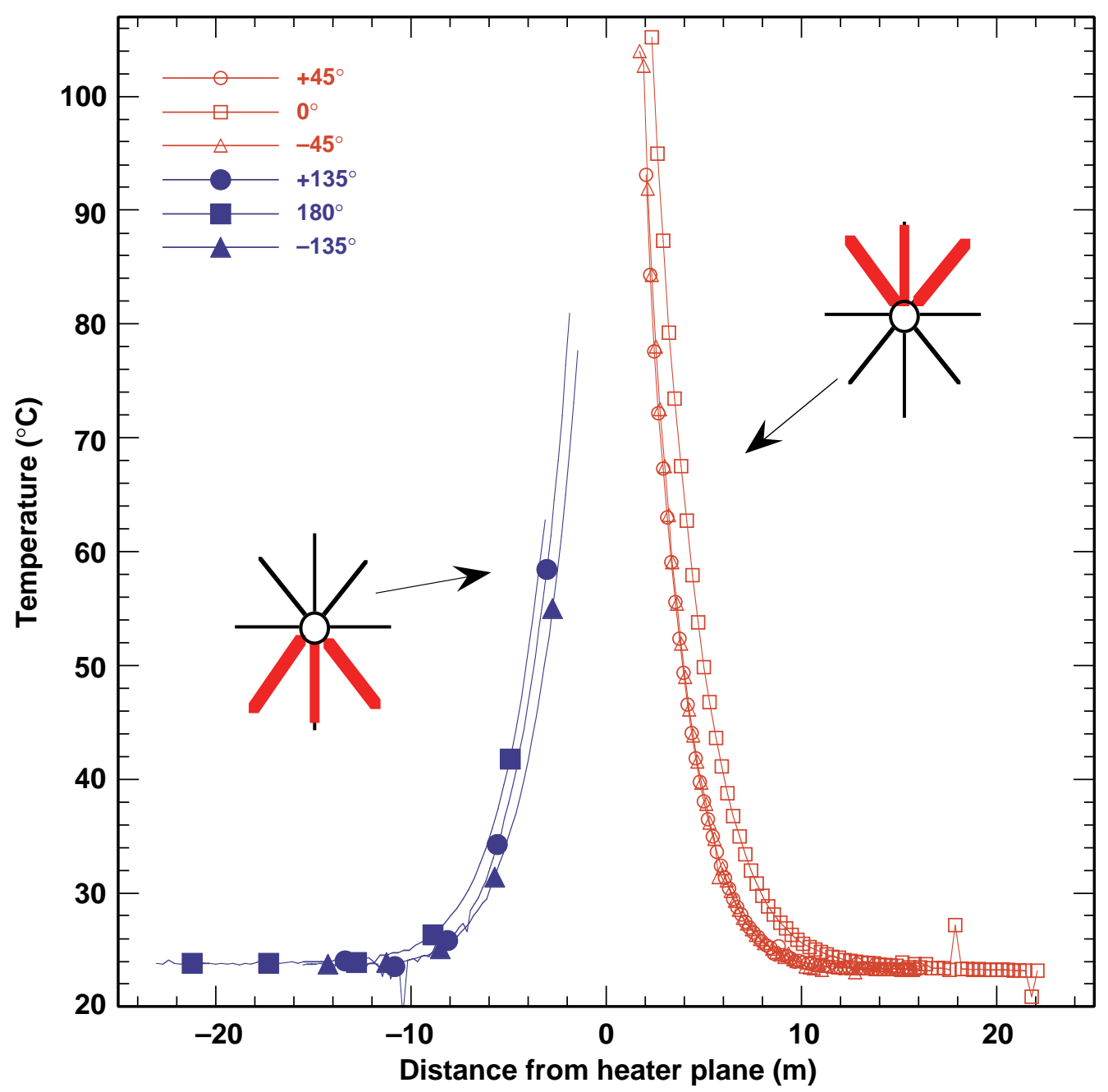

Figure 11. Temperatures for boreholes 137 through 144 on February 28, 1998. 


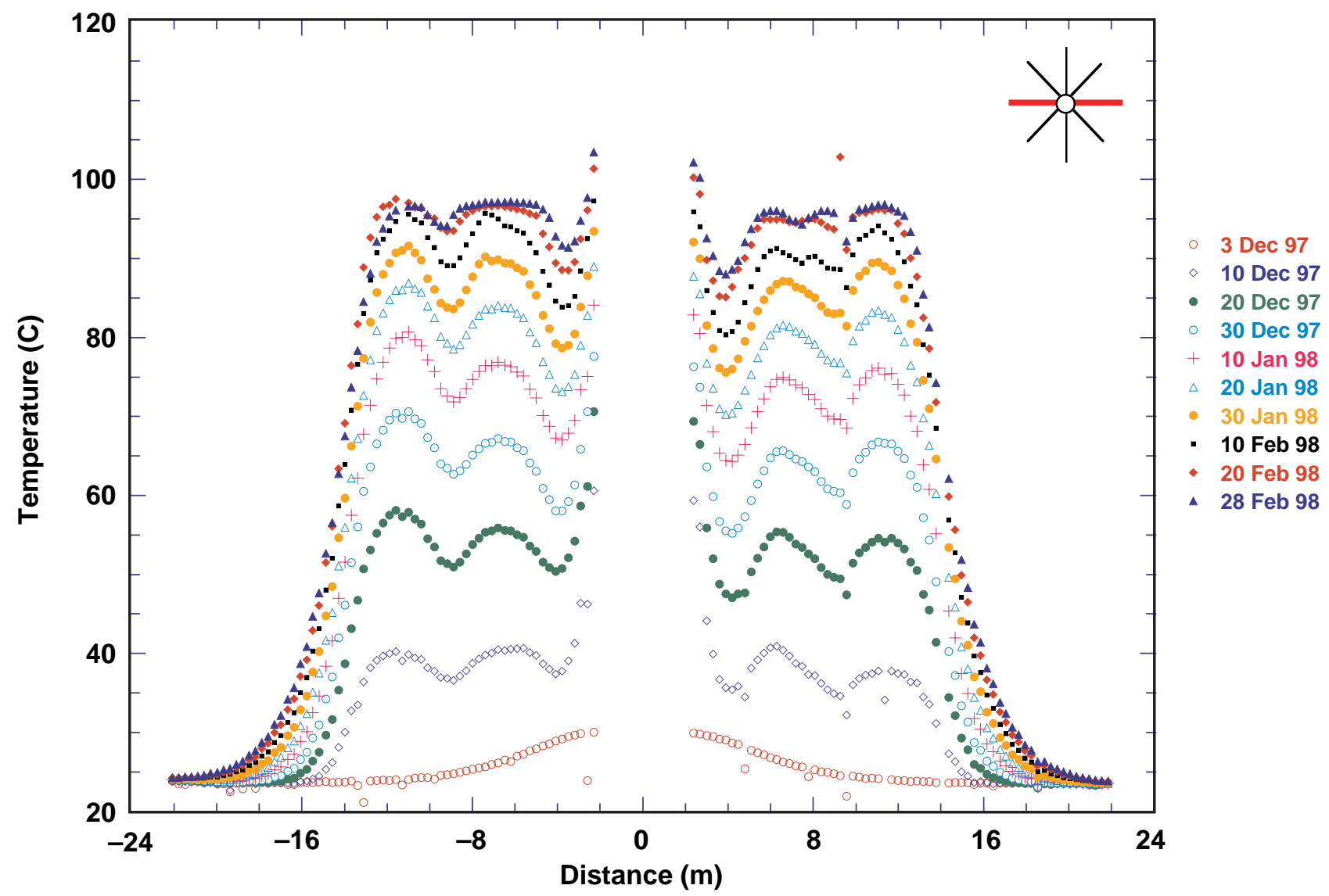

Figure 12. Temperature history for horizontal boreholes 139 and 143. 


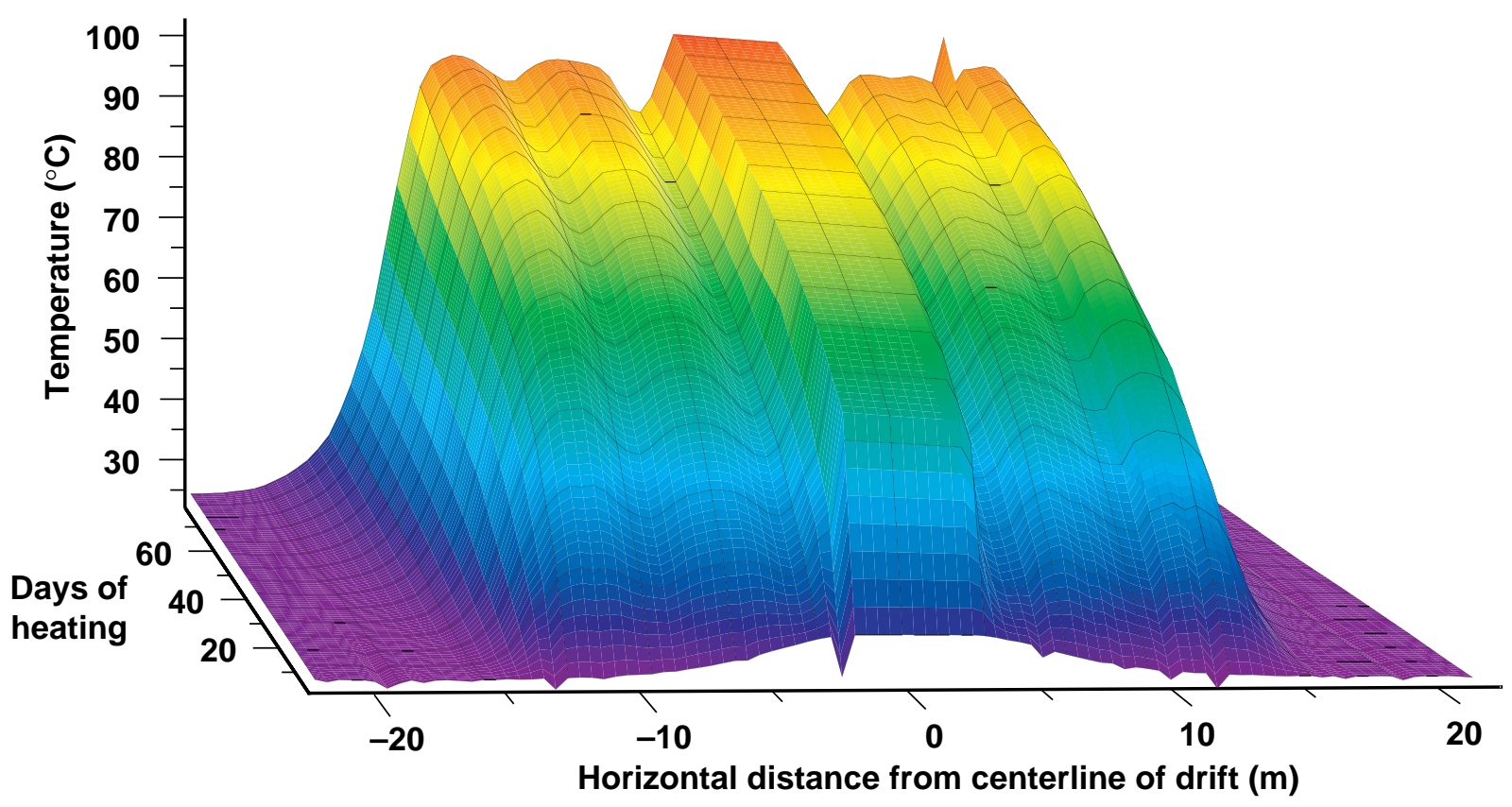

Figure 13. Temperature data from boreholes 139 and 143 shown as a surface. 


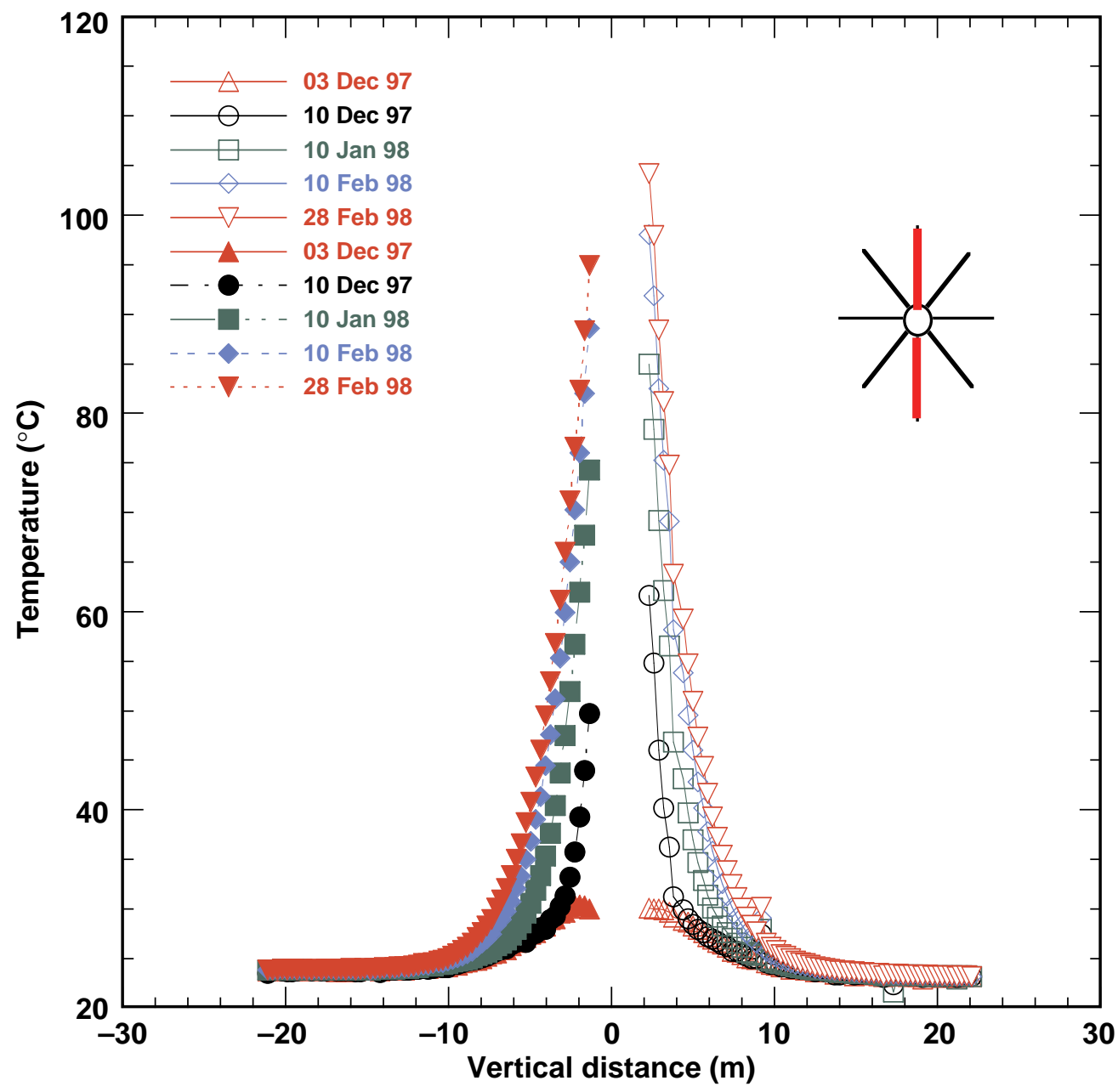

Figure 14. Temperature in boreholes 158 and 162 at various times. 


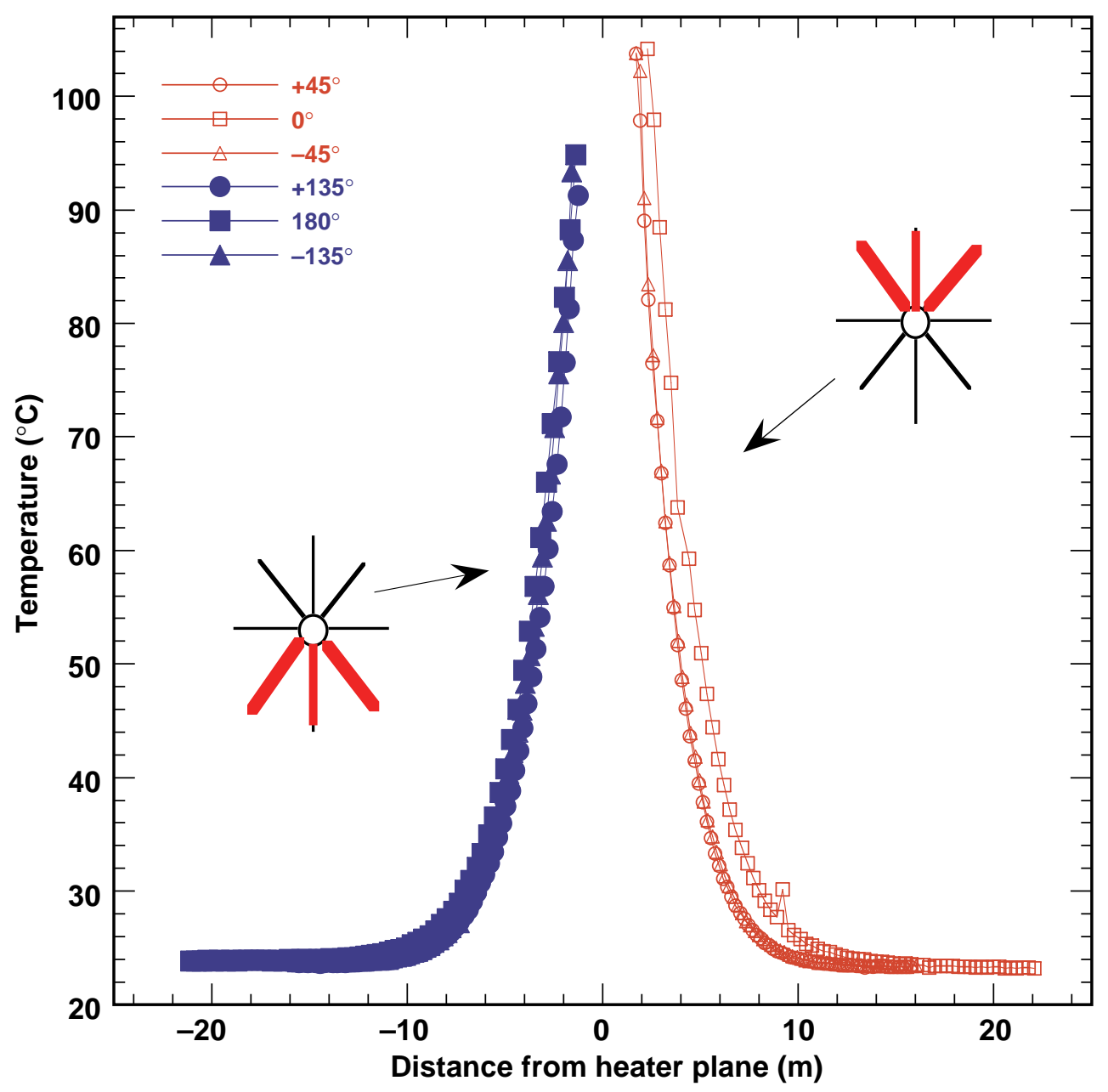

Figure 15. Temperatures on February 28, 1998, for boreholes 158 through 165. 


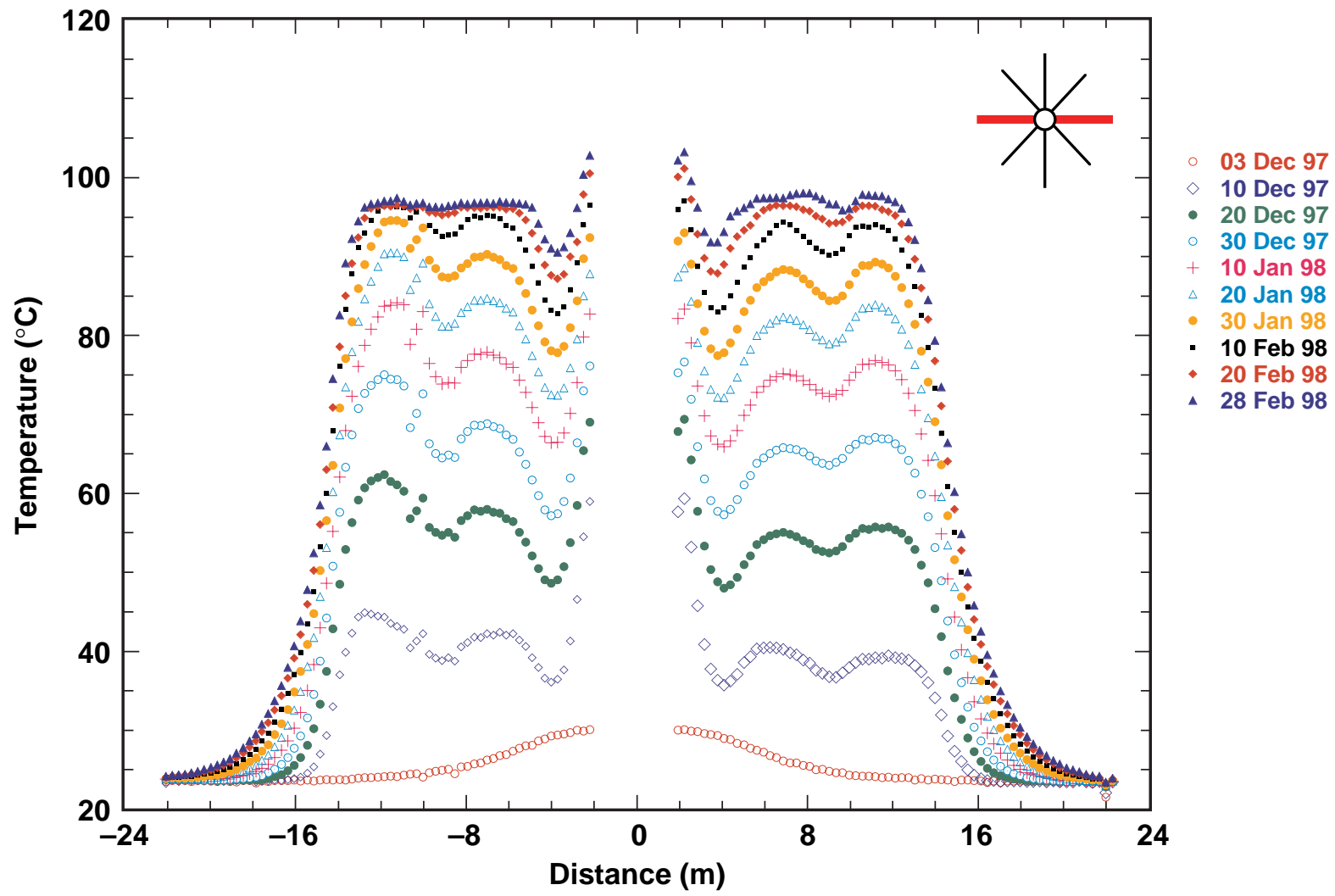

Figure 16. Temperature history for boreholes 160 and 164 . 

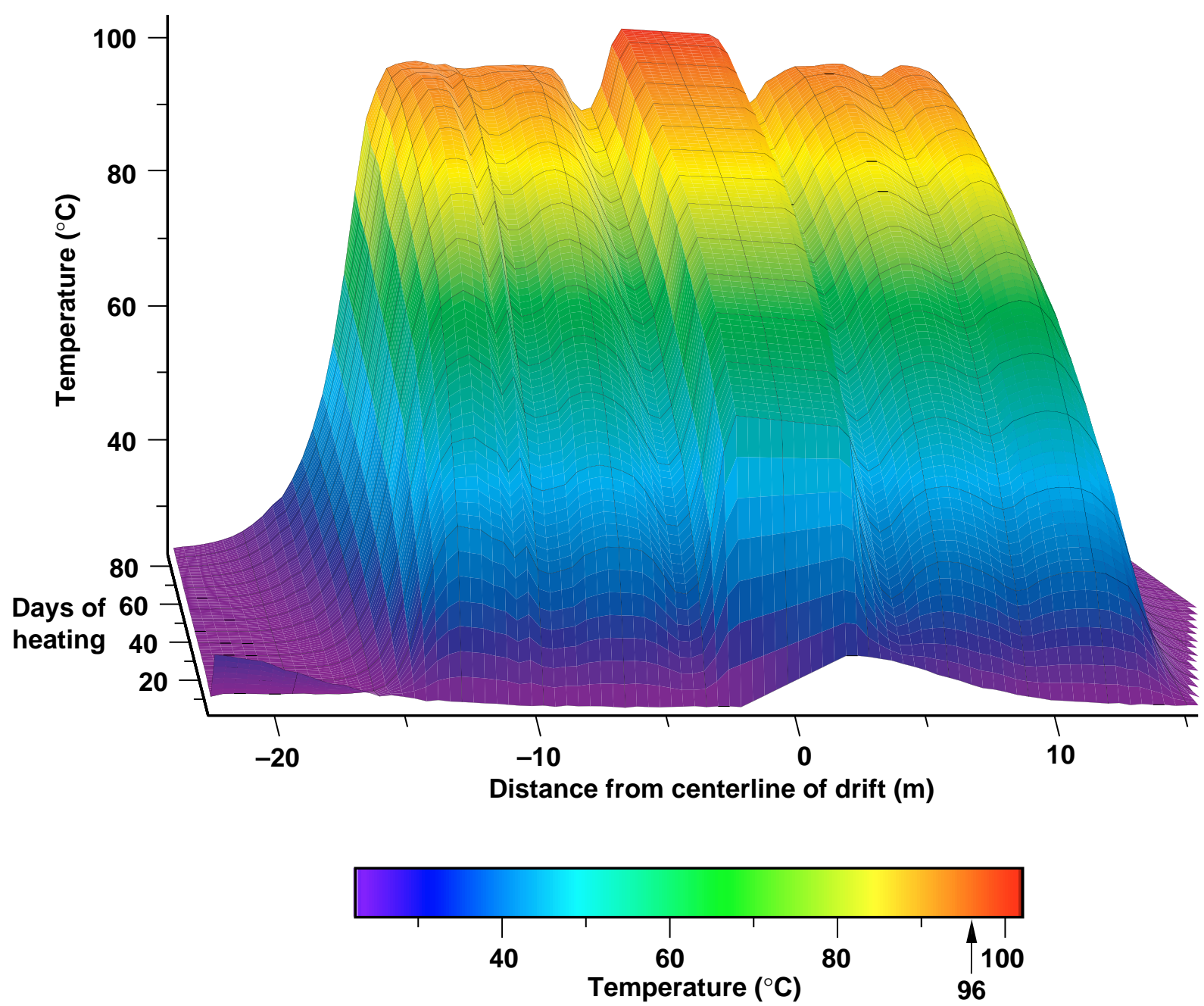

Figure 17. Temperature data from boreholes 160 and 164 shown as a surface. 


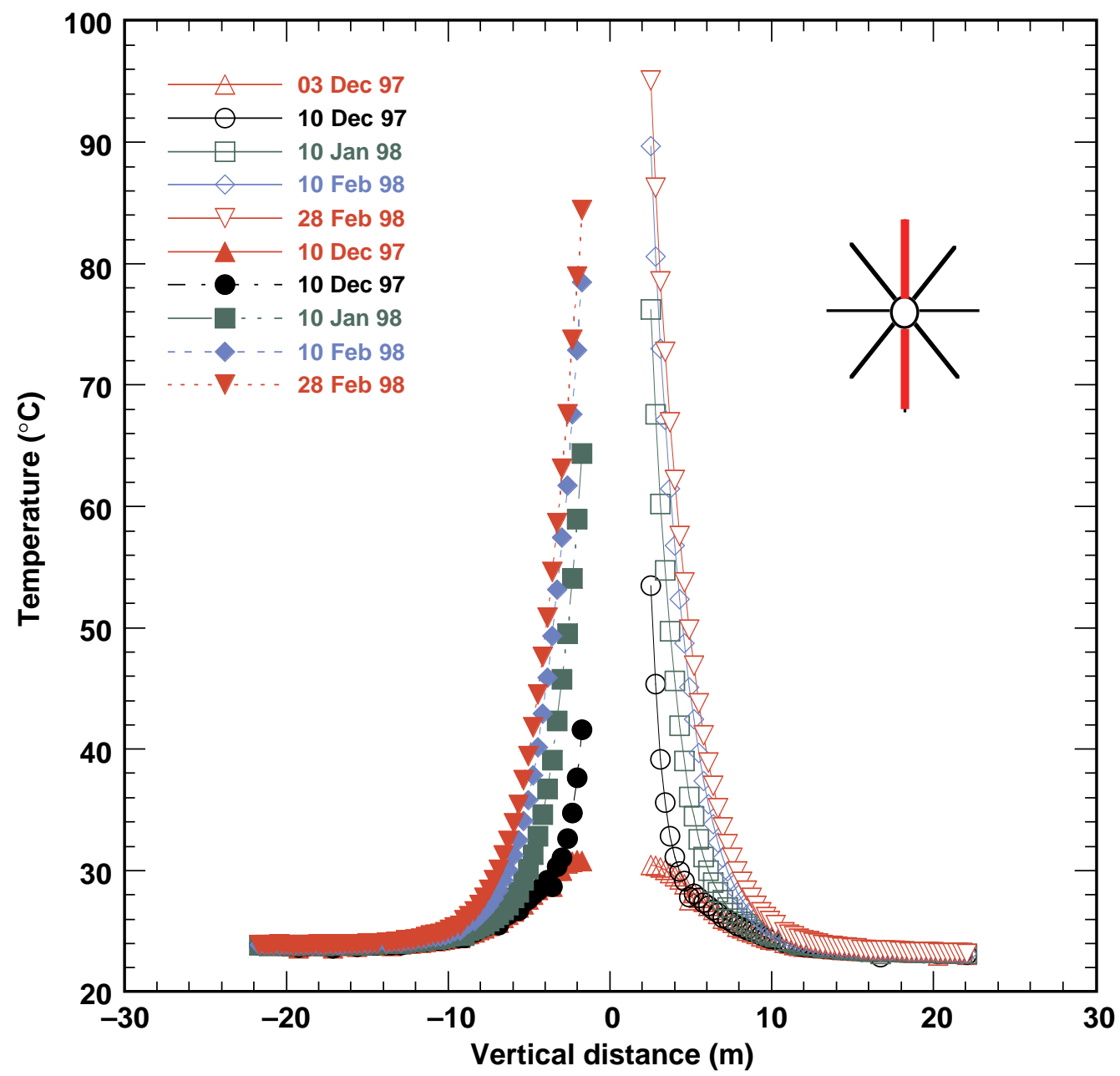

Figure 18. Temperature in boreholes 170 and 173 for various times. 


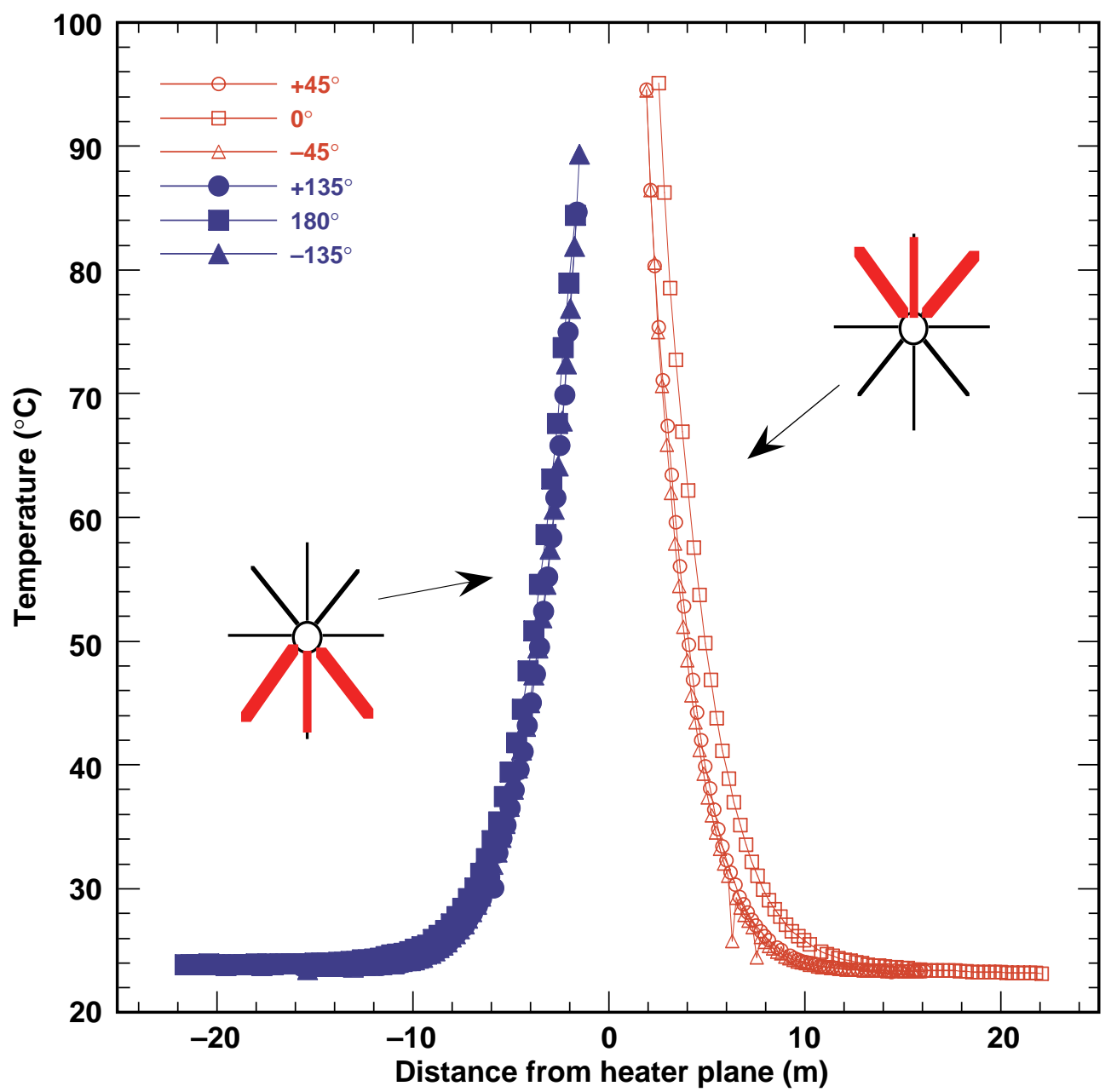

Figure 19. Temperature for boreholes 170 through 175 on February 28, 1998. 


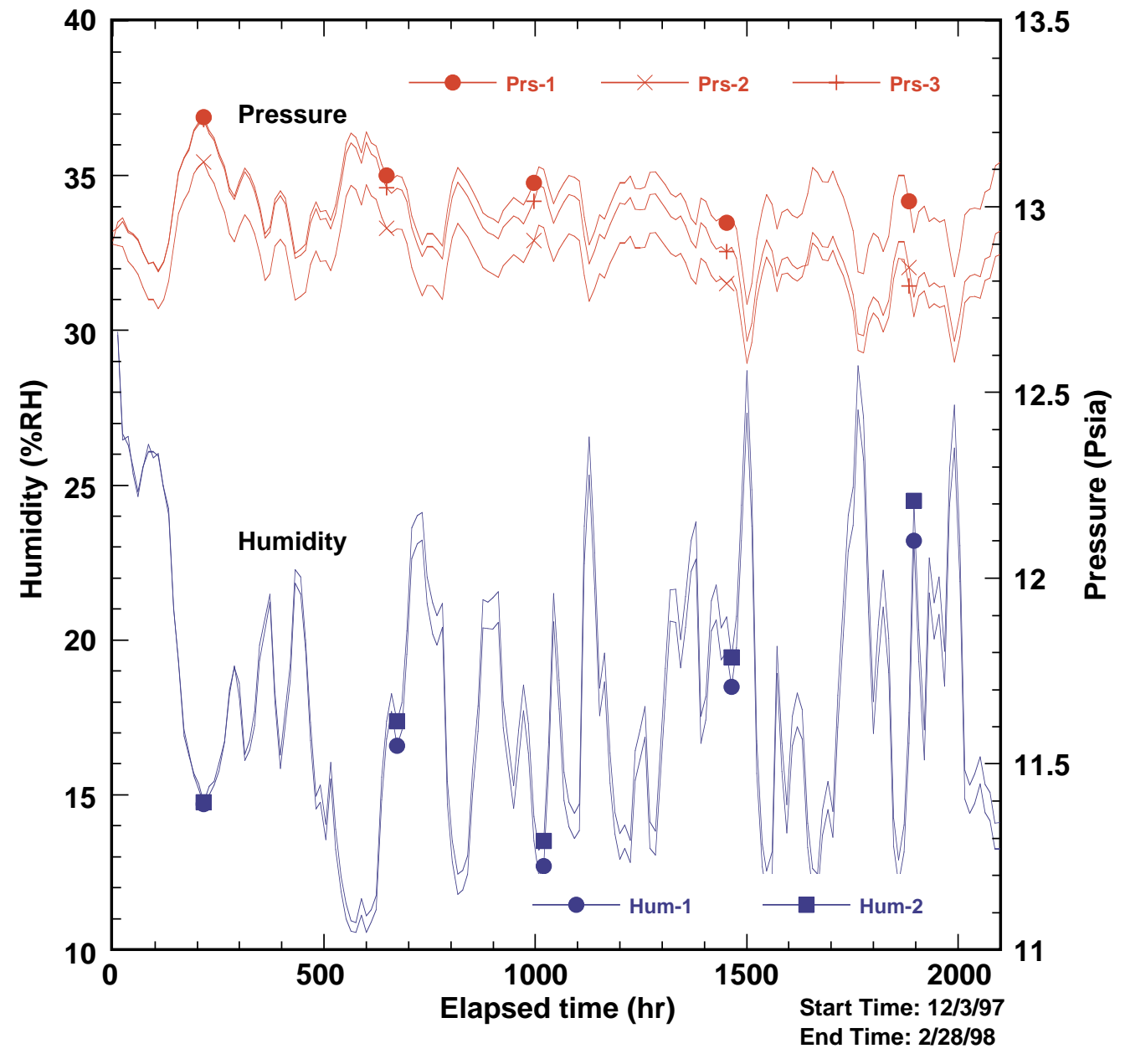

Figure 20. Relative humidity data, along with air pressure, for two sensors. 


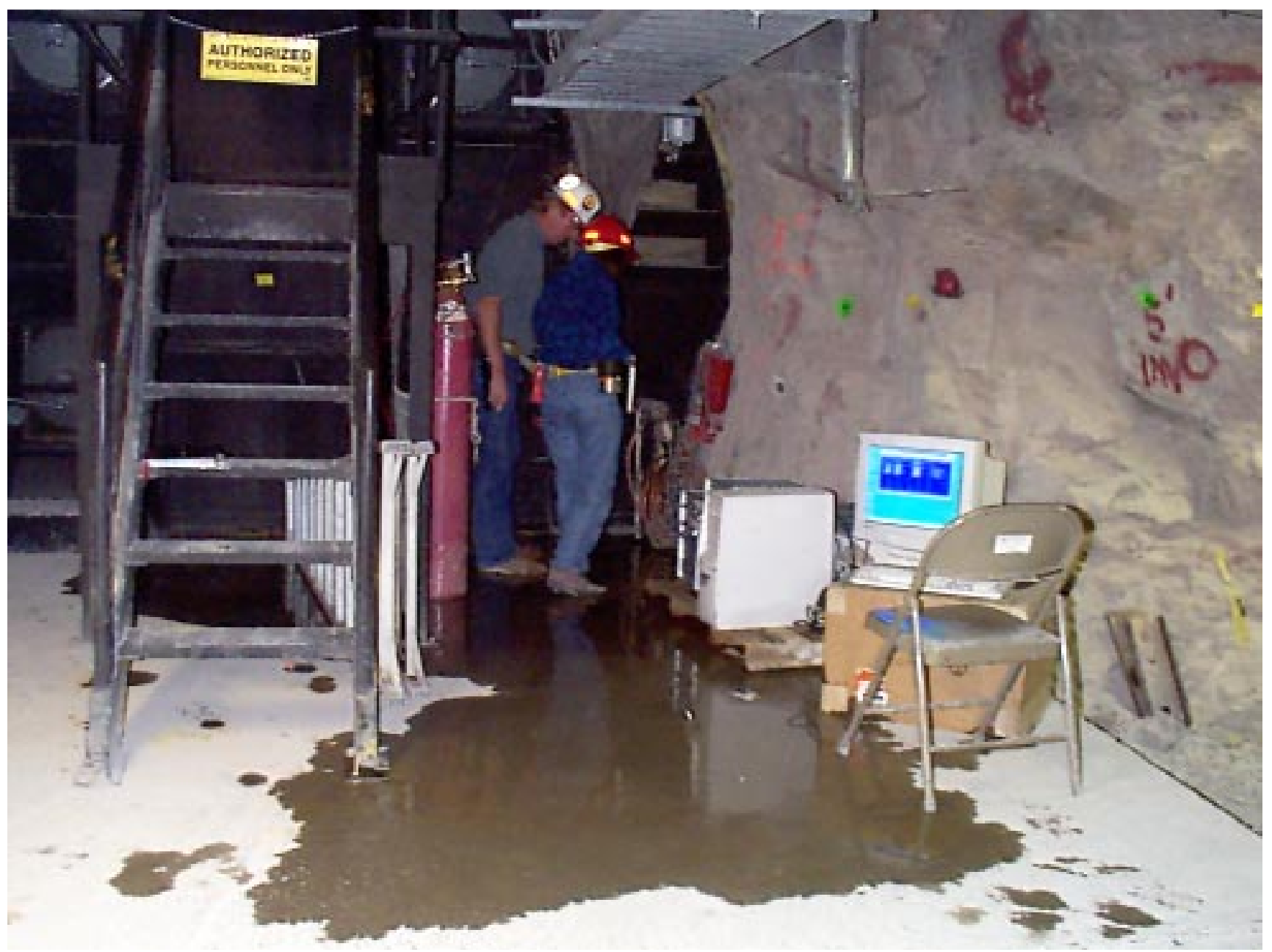

Figure 21. Condensation observed on bulkhead; puddle on floor in front of bulkhead. 


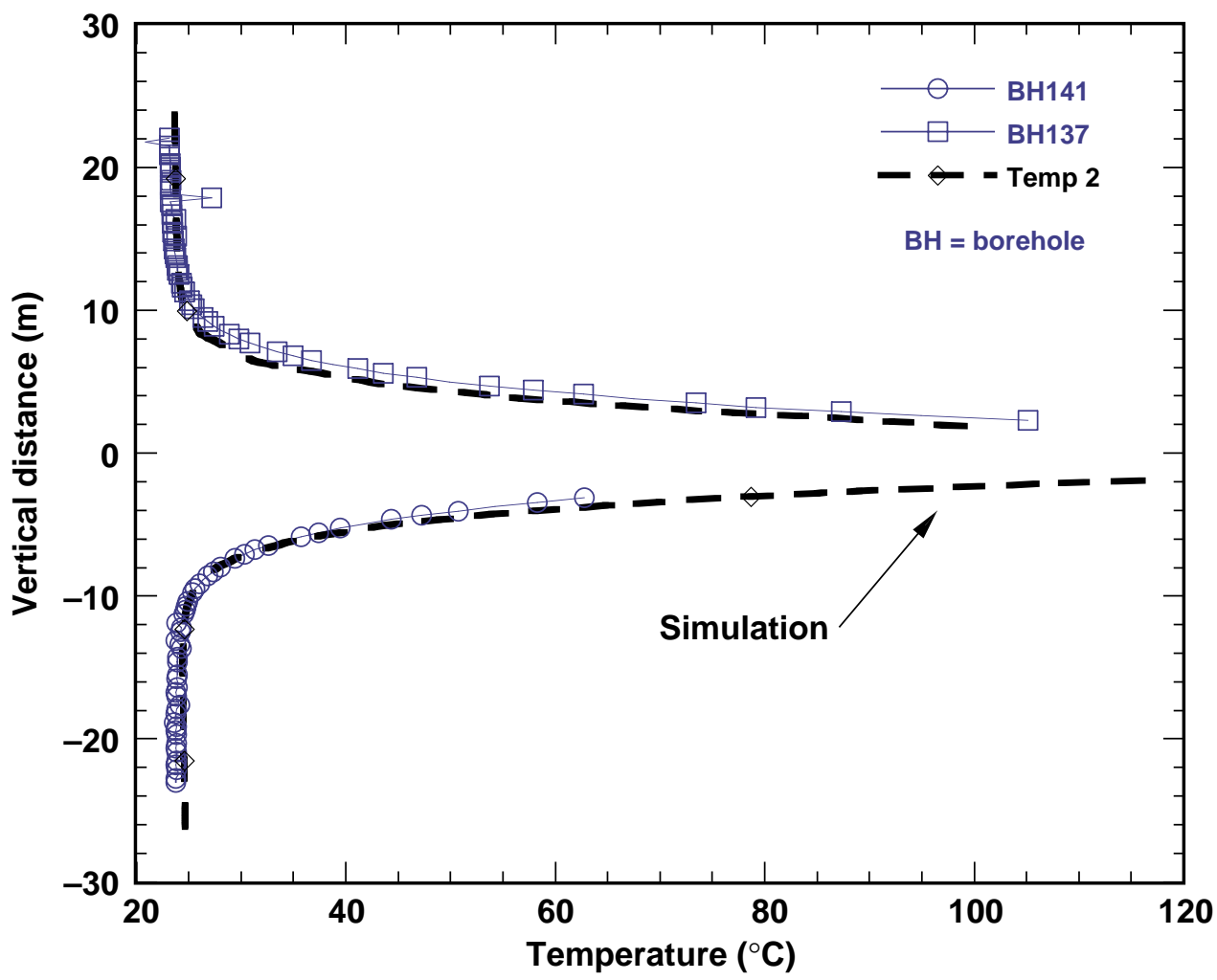

Figure 22. Comparison of measured and simulated temperatures along a vertical profile $12 \mathrm{~m}$ from the bulkhead. 
Appendix A 



\section{Appendix A}

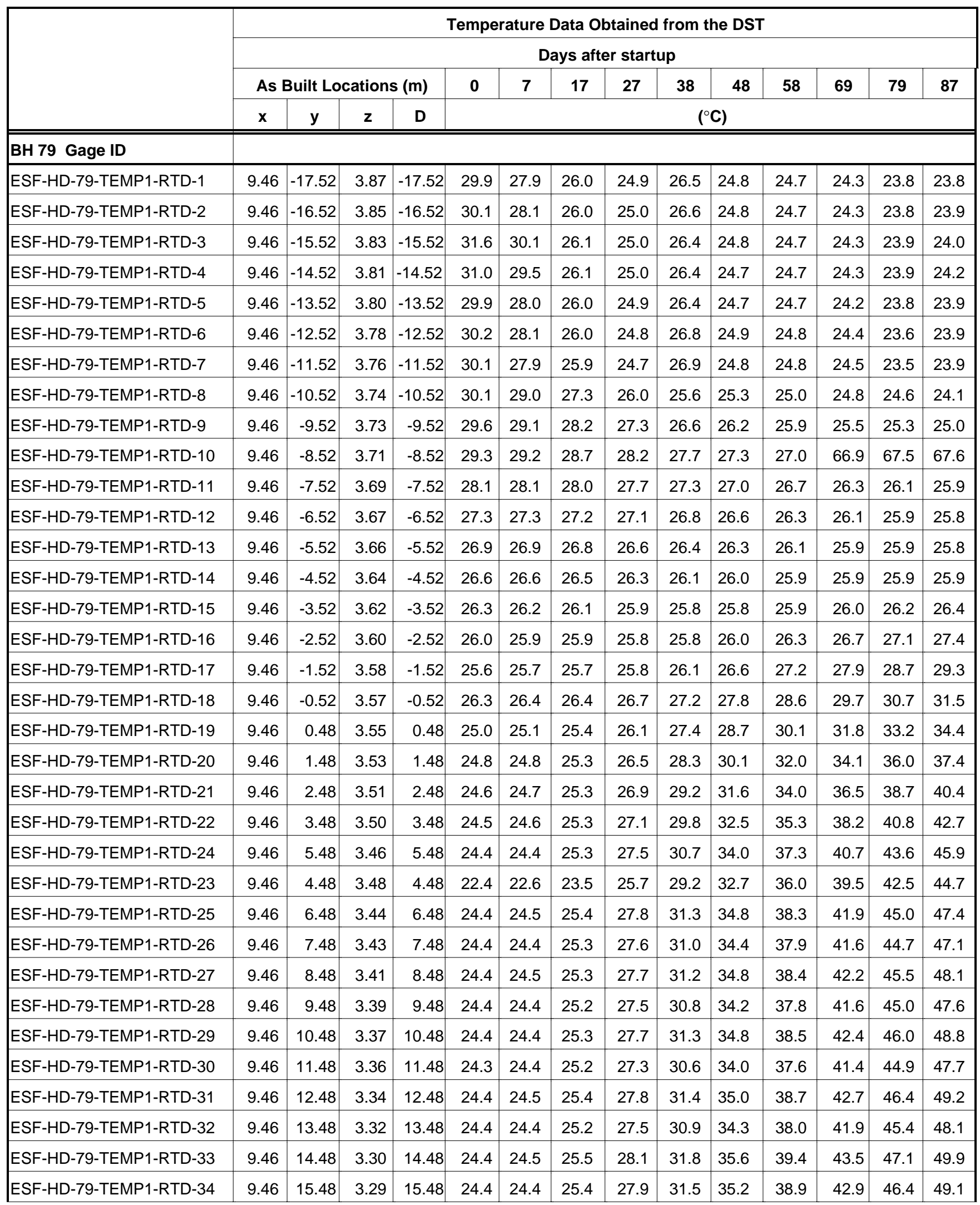




\begin{tabular}{|c|c|c|c|c|c|c|c|c|c|c|c|c|c|c|}
\hline \multirow{5}{*}{ ESF-HD-79-TEMP1-RTD-35 } & \multicolumn{14}{|c|}{ Temperature Data Obtained from the DST } \\
\hline & \multicolumn{14}{|c|}{ Days after startup } \\
\hline & \multicolumn{4}{|c|}{ As Built Locations (m) } & 0 & 7 & 17 & 27 & 38 & 48 & 58 & 69 & 79 & 87 \\
\hline & $x$ & y & $\mathbf{z}$ & D & \multicolumn{10}{|c|}{$\left({ }^{\circ} \mathrm{C}\right)$} \\
\hline & 9.46 & 16.48 & 3.27 & 16.48 & 24.5 & 24.6 & 25.8 & 28.7 & 32.7 & 36.6 & 40.5 & 44.6 & 48.2 & 50.9 \\
\hline ESF-HD-79-TEMP1-RTD-36 & & 17.48 & & 17.48 & 24.4 & 24.5 & & & 32.2 & 35.9 & 39.7 & 43.7 & 47.2 & 9.9 \\
\hline ESF-HD-79-TEMP1-RTD-37 & & 8.48 & & 18.48 & 24.4 & & & & 33.4 & & & 45.4 & 49.0 & 1.7 \\
\hline ESF-HD-79-TEMP1-RTD-38 & & 9.48 & & 19.48 & 24.4 & & & & 33.0 & & 40.6 & 44.6 & 48.1 & \\
\hline ESF-HD-79-TEMP1-RTD-39 & & & & 20.48 & 24.4 & & & & 34.1 & & & 46.4 & 50.1 & \\
\hline ESF-HD-79-TEMP1-R & & & & 21.48 & 24.4 & & 26.0 & & 33.4 & & & 45.5 & 49.1 & \\
\hline ESF-HD-7S & & . & & 22.48 & 24.5 & 24.6 & 26.3 & & 34.4 & & & 47.1 & 50.9 & \\
\hline ESF-HD-79 & & & & 23.48 & 24.4 & 24.5 & & & 33.9 & 38.1 & 然 & 46.5 & 50.2 & \\
\hline ESF-HD-7 & & 管 & & 24.48 & 24.5 & & & & & & & 48.2 & 52.1 & \\
\hline ESF-HD-79-TEMP1- & & & & 25.48 & 24.4 & & & & & & & 47.6 & & \\
\hline ESF-HD-79-TEMP1-I & & 年 & 3.09 & 26.48 & 24.5 & 24.6 & & & & & & 49.8 & & \\
\hline ESF-HD-79-TEMP1-I & & Whe & & 27.48 & 24.5 & & & & & & & 49.5 & & \\
\hline ESF-HD-7 & & 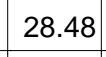 & & 28.48 & & & & & & & & & & \\
\hline & & . & & 29.48 & & & & & & & & & & \\
\hline & & & & & & & & & & & & & & \\
\hline & & & & & & & & & & & & 10 & & \\
\hline & & & & & & & & & & & & & & \\
\hline & & & & & & & & & & & & & & \\
\hline ESF-HD-79-TEMP1-RTD-53 & & & & 34.48 & 24.5 & & & & & & & & & \\
\hline ESF-HD-79-TEMP1-RTD-54 & & & & & 23.2 & & & & & & & & & \\
\hline ESF-HD-79-TEMP1-RTD-55 & & & 2.92 & 36.48 & 24.6 & & & & & & & 61 & & \\
\hline ESF-HD-79-TEMP1-RTD-56 & & & 2.90 & 37.48 & 24.5 & & & & & & & & & \\
\hline ESF-HD-79-TEMP1-RTD-57 & & & 2.88 & 38.48 & & & & & & & & & & \\
\hline & & & & & & & & & & & & & & \\
\hline & & & & & & & & & & & & & & \\
\hline ESF-HD-79-TEMP1-RTD-60 & & 41.48 & 2.83 & 41.48 & 25.7 & 26.4 & 31.4 & 37.4 & 43.9 & 49.3 & & 9.4 & 64.2 & 8.5 \\
\hline \multicolumn{15}{|l|}{ BH 80 Gage ID } \\
\hline 1 & -9.48 & -11.37 & 3.23 & -11.37 & 29.5 & 25.7 & & & 24.9 & 23.7 & & 22.8 & 22.6 & 3.7 \\
\hline ESF-HD-80-TEMP2-RTD-2 & -9.49 & -10.37 & 3.23 & -10.37 & 29.4 & 28.3 & 26.6 & 25.3 & 26.4 & 24.8 & 24.5 & 24.2 & 24.1 & 23.9 \\
\hline ESF-HD-80-TEMP2-RTD-3 & -9.50 & -9.37 & 3.23 & -9.37 & 28.7 & 28.5 & 26.8 & 27.5 & 26.4 & 25.9 & 25.6 & 25.3 & 25.1 & 25.5 \\
\hline ESF-HD-80-TEMP2-RTD-4 & -9.50 & -8.37 & 3.22 & -8.37 & 28.2 & 28.9 & 27.4 & 27.2 & 26.6 & 26.5 & 26.0 & 25.9 & 25.6 & 4.9 \\
\hline ESF-HD-80-TEMP2-RTD-5 & -9.51 & -7.38 & 3.22 & -7.38 & 27.5 & 27.5 & 27.9 & 27.1 & 26.9 & 26.5 & 26.0 & 26.1 & 25.9 & 6.0 \\
\hline ESF-HD-80-TEMP2-RTD-6 & -9.52 & -6.38 & 3.22 & -6.38 & 26.7 & 26.8 & 28.0 & 26.8 & 26.9 & 26.6 & 26.2 & 26.1 & 26.1 & 25.8 \\
\hline ESF-HD-80-TEMP2-RTD-7 & -9.53 & -5.38 & 3.22 & -5.38 & 25.9 & 26.0 & 26.1 & 26.2 & 26.2 & 26.2 & 26.2 & 26.2 & 26.2 & 26.5 \\
\hline ESF-HD-80-TEMP2-RTD-8 & & -4.38 & 3.22 & -4.38 & 25.4 & & 25.7 & 25.8 & 25.9 & 25.9 & 26.3 & 26.2 & 26.3 & 26.2 \\
\hline ESF-HD-80-TEMP2-RTD-9 & -9.54 & -3.38 & 3.22 & -3.38 & 25.0 & 25.1 & 25.2 & 25.3 & 25.4 & 25.9 & 26.3 & 26.4 & 26.8 & 28.2 \\
\hline ESF-HD-80-TEMP2-RTD-10 & -9.55 & -2.38 & 3.22 & -2.38 & 24.7 & 24.8 & 24.9 & 25.3 & 25.5 & 25.8 & 26.4 & 27.0 & 27.7 & 27.1 \\
\hline
\end{tabular}


First Quarter Report of Temperature Measurements During the Heating Phase of the Drift-Scale Test

\begin{tabular}{|c|c|c|c|c|c|c|c|c|c|c|c|c|c|c|}
\hline \multirow{5}{*}{\begin{tabular}{|l} 
\\
ESF-HD-80-TEMP2-RTD-11 \\
\end{tabular}} & \multicolumn{14}{|c|}{ Temperature Data Obtained from the DST } \\
\hline & \multicolumn{14}{|c|}{ Days after startup } \\
\hline & \multicolumn{4}{|c|}{ As Built Locations (m) } & 0 & 7 & 17 & 27 & 38 & 48 & 58 & 69 & 79 & 87 \\
\hline & $\mathbf{x}$ & $\mathbf{y}$ & $\mathbf{z}$ & D & \multicolumn{10}{|c|}{$\left({ }^{\circ} \mathbf{C}\right)$} \\
\hline & -9.55 & -1.39 & 3.22 & -1.39 & 24.6 & 24.6 & 24.7 & 25.1 & 25.7 & 26.4 & 27.3 & 28.3 & 29.2 & 32.3 \\
\hline ESF-HD-80-TEMP2-RTD-12 & -9.56 & -0.39 & 3.22 & -0.39 & 24.4 & 24.5 & 24.8 & 25.1 & 26.3 & 27.4 & 28.6 & 30.0 & 31.3 & 30.0 \\
\hline ESF-HD-80-TEMP2-RTD-13 & -9.57 & 0.61 & 3.21 & 0.61 & 24.4 & 24.5 & 24.8 & 25.9 & 27.5 & 29.2 & 30.9 & 32.8 & 34.5 & 38.9 \\
\hline ESF-HD-80-TEMP2-RTD-14 & -9.57 & 1.61 & 3.21 & 1.61 & 24.4 & 24.5 & 25.0 & 26.4 & 28.6 & 30.7 & 32.9 & 35.2 & 37.3 & 35.8 \\
\hline ESF-HD-80-TEMP2-RTD-15 & -9.58 & 2.61 & 3.21 & 2.61 & 24.4 & 24.5 & 25.2 & 27.1 & 29.8 & 32.4 & 35.0 & 37.8 & 40.2 & 44.1 \\
\hline ESF-HD-80-TEMP2-RTD-16 & -9.59 & 3.60 & 3.21 & 3.60 & 24.4 & 24.5 & 25.2 & 27.3 & 30.3 & 33.2 & 36.2 & 39.3 & 42.0 & 42.1 \\
\hline ESF-HD-80-TEMP2-RTD-17 & -9.60 & 4.60 & 3.21 & 4.60 & 24.4 & 24.5 & 25.2 & 27.5 & 30.8 & 34.0 & 37.2 & 40.6 & 43.6 & 46.5 \\
\hline ESF-HD-80-TEMP2-RTD-18 & -9.60 & 5.60 & 3.21 & 5.60 & 24.4 & 24.5 & 25.3 & 27.5 & 30.8 & 34.0 & 37.4 & 41.0 & 44.1 & 45.9 \\
\hline ESF-HD-80-TEMP2-RTD-19 & -9.61 & 6.60 & 3.21 & 6.60 & 24.4 & 24.5 & 25.3 & 27.5 & 30.8 & 34.2 & 37.7 & 41.5 & 44.8 & 47.5 \\
\hline ESF-HD-80-TEMP2-RTD-20 & -9.62 & 7.60 & 3.21 & 7.60 & 24.4 & 24.4 & 25.3 & 27.6 & 30.9 & 34.3 & 37.8 & 41.5 & 44.9 & 47.4 \\
\hline ESF-HD-80-TEMP2-RTD-21 & -9.62 & 8.59 & 3.21 & 8.59 & 24.4 & 24.4 & 25.3 & 27.7 & 31.2 & 34.5 & 38.0 & 41.9 & 45.3 & 48.2 \\
\hline ESF-HD-80-TEMP2-RTD-22 & -9.63 & 9.59 & 3.21 & 9.59 & 24.4 & 24.4 & 25.4 & 27.6 & 31.1 & 34.6 & 38.1 & 42.0 & 45.5 & 48.1 \\
\hline ESF-HD-80-TEMP2-RTD-23 & -9.64 & 10.59 & 3.20 & 10.59 & 24.4 & 24.4 & 25.4 & 27.8 & 31.3 & 34.6 & 38.2 & 42.1 & 45.5 & 48.5 \\
\hline ESF-HD-80-TEMP2-RTD-24 & -9.64 & 11.59 & 3.20 & 11.59 & 24.4 & 24.4 & 25.3 & 27.6 & 31.0 & 34.7 & 38.3 & 42.4 & 45.6 & 47.9 \\
\hline ESF-HD-80-TEMP2-RTD-25 & -9.65 & 12.59 & 3.20 & 12.59 & 24.3 & 24.4 & 25.3 & 27.6 & 31.1 & 34.9 & 38.5 & 42.1 & 45.9 & 48.2 \\
\hline ESF-HD-80-TEMP2-RTD-26 & -9.66 & 13.58 & 3.20 & 13.58 & 24.4 & 24.4 & 25.2 & 27.5 & 30.9 & 34.3 & 37.9 & 41.7 & 45.1 & 47.7 \\
\hline ESF-HD-80-TEMP2-RTD-27 & -9.67 & 14.58 & 3.20 & 14.58 & 24.4 & 24.4 & 25.3 & 27.5 & 31.0 & 34.4 & 38.0 & 41.9 & 45.4 & 48.1 \\
\hline ESF-HD-80-TEMP2-RTD-28 & -9.67 & 15.58 & 3.20 & 15.58 & 24.4 & 24.4 & 25.3 & 27.6 & 31.0 & 34.5 & 38.1 & 42.0 & 45.5 & 48.2 \\
\hline ESF-HD-80-TEMP2-RTD-29 & -9.68 & 16.58 & 3.20 & 16.58 & 24.4 & 24.4 & 25.3 & 27.6 & 31.0 & 34.4 & 38.0 & 41.8 & 45.3 & 48.1 \\
\hline ESF-HD-80-TEMP2-RTD-30 & -9.69 & 17.58 & 3.20 & 17.58 & 24.4 & 24.4 & 25.3 & 27.6 & 31.0 & 34.4 & 37.9 & 41.8 & 45.3 & 48.0 \\
\hline ESF-HD-80-TEMP2-RTD-31 & -9.69 & 18.58 & 3.20 & 18.58 & 24.4 & 24.5 & 25.2 & 27.3 & 30.6 & 34.0 & 37.5 & 41.4 & 44.8 & 47.7 \\
\hline ESF-HD-80-TEMP2-RTD-32 & -9.70 & 19.57 & 3.19 & 19.57 & 24.4 & 24.5 & 25.3 & 27.5 & 30.8 & 34.2 & 37.7 & 41.6 & 45.1 & 47.9 \\
\hline ESF-HD-80-TEMP2-RTD-33 & -9.71 & 20.57 & 3.19 & 20.57 & 24.4 & 24.5 & 25.3 & 27.6 & 31.0 & 34.4 & 38.0 & 41.8 & 45.3 & 48.1 \\
\hline ESF-HD-80-TEMP2-RTD-34 & -9.71 & 21.57 & 3.19 & 21.57 & 24.4 & 24.5 & 25.2 & 27.4 & 30.7 & 34.1 & 37.6 & 41.4 & 44.9 & 47.7 \\
\hline ESF-HD-80-TEMP2-RTD-35 & -9.72 & 22.57 & 3.19 & 22.57 & 24.5 & 24.5 & 25.4 & 27.6 & 31.0 & 34.4 & 38.0 & 41.9 & 45.3 & 48.0 \\
\hline ESF-HD-80-TEMP2-RTD-36 & -9.73 & 23.57 & 3.19 & 23.57 & 24.4 & 24.5 & 25.4 & 27.6 & 31.0 & 34.4 & 38.0 & 41.8 & 45.3 & 48.0 \\
\hline ESF-HD-80-TEMP2-RTD-37 & -9.74 & 24.56 & 3.19 & 24.56 & 24.5 & 24.5 & 25.2 & 27.2 & 30.4 & 33.7 & 37.2 & 41.0 & 44.3 & 47.0 \\
\hline ESF-HD-80-TEMP2-RTD-38 & -9.74 & 25.56 & 3.19 & 25.56 & 24.4 & 24.5 & 25.2 & 27.3 & 30.5 & 33.9 & 37.4 & 41.2 & 44.6 & 47.3 \\
\hline ESF-HD-80-TEMP2-RTD-39 & -9.75 & 26.56 & 3.19 & 26.56 & 24.4 & 24.5 & 25.2 & 27.4 & 30.7 & 34.0 & 37.5 & 41.3 & 44.5 & 47.1 \\
\hline ESF-HD-80-TEMP2-RTD-40 & -9.76 & 27.56 & 3.19 & 27.56 & 24.4 & 24.5 & 25.2 & 27.3 & 30.7 & 34.0 & 37.5 & 41.4 & 44.7 & 47.3 \\
\hline ESF-HD-80-TEMP2-RTD-41 & -9.76 & 28.56 & 3.18 & 28.56 & 24.4 & 24.4 & 25.1 & 27.2 & 30.4 & 33.7 & 37.1 & 40.8 & 44.1 & 46.6 \\
\hline ESF-HD-80-TEMP2-RTD-42 & -9.77 & 29.55 & 3.18 & 29.55 & 24.3 & 24.4 & 25.2 & 27.3 & 30.6 & 34.0 & 37.4 & 41.1 & 44.4 & 46.8 \\
\hline ESF-HD-80-TEMP2-RTD-43 & -9.78 & 30.55 & 3.18 & 30.55 & 24.4 & 24.5 & 25.2 & 27.4 & 30.7 & 34.0 & 37.5 & 41.3 & 44.5 & 47.1 \\
\hline ESF-HD-80-TEMP2-RTD-44 & -9.78 & 31.55 & 3.18 & 31.55 & 24.4 & 24.4 & 25.1 & 27.2 & 30.4 & 33.7 & 37.1 & 40.8 & 44.1 & 46.6 \\
\hline ESF-HD-80-TEMP2-RTD-45 & -9.79 & 32.55 & 3.18 & 32.55 & 24.4 & 24.4 & 25.2 & 27.3 & 30.5 & 33.7 & 37.1 & 40.7 & 43.9 & 46.4 \\
\hline ESF-HD-80-TEMP2-RTD-46 & -9.80 & 33.55 & 3.18 & 33.55 & 24.4 & 24.4 & 25.2 & 27.3 & 30.5 & 33.7 & 37.1 & 40.7 & 43.9 & 46.5 \\
\hline ESF-HD-80-TEMP2-RTD-47 & -9.80 & 34.55 & 3.18 & 34.55 & 24.3 & 24.4 & 25.0 & 26.9 & 30.0 & 33.2 & 36.4 & 40.0 & 43.2 & 45.6 \\
\hline
\end{tabular}




\begin{tabular}{|c|c|c|c|c|c|c|c|c|c|c|c|c|c|c|}
\hline & \multicolumn{14}{|c|}{ Temperature Data Obtained from the DST } \\
\hline & \multicolumn{14}{|c|}{ Days after startup } \\
\hline & \multicolumn{4}{|c|}{ As Built Locations (m) } & 0 & 7 & 17 & 27 & 38 & 48 & 58 & 69 & 79 & 87 \\
\hline & $\mathbf{x}$ & y & $\mathbf{z}$ & D & \multicolumn{10}{|c|}{$\left({ }^{\circ} \mathrm{C}\right)$} \\
\hline ESF-HD-80-TEMP2-RTD-48 & -9.81 & 35.54 & 3.18 & 35.54 & 24.3 & 24.4 & 25.1 & 27.1 & 30.2 & 33.4 & 36.8 & 40.4 & 43.6 & 46.0 \\
\hline ESF-HD-80-TEMP2-RTD-49 & -9.82 & 6.54 & 3.18 & 36.54 & 24.3 & 24.4 & 25.1 & 7.1 & 0.2 & 33.5 & 36.8 & 0.3 & 43.5 & 5.9 \\
\hline ESF-HD-80-TEMP2-RTD-50 & -9.83 & 7.54 & 3.17 & 37.54 & 24.3 & 24.3 & 25.2 & 7.5 & 30.8 & 34.1 & 37.5 & 1.1 & 44.3 & 46.8 \\
\hline ESF-HD-80-TEMP2-RTD-51 & -9.83 & 8.54 & 3.17 & 38.54 & 24.3 & 24.4 & 25.2 & 27.5 & 30.8 & 34.1 & 37.4 & 41.0 & 44.1 & 46.5 \\
\hline ESF-HD-80-TEMP2-RTD-52 & -9.84 & 9.54 & 3.17 & 39.54 & 24.3 & 24.4 & 25.3 & 27.8 & 31.3 & 34.7 & 38.0 & 41.6 & 44.7 & 47.1 \\
\hline ESF-HD-80-TEMP2-RTD-53 & -9.85 & .53 & 3.17 & 40.53 & 24.3 & 24.3 & 25.3 & 27.8 & 31.4 & 34.7 & 38.0 & 41.5 & 44.5 & 46.9 \\
\hline ESF-HD-80-TEMP2-RTD-54 & -9.85 & 1.53 & 3.17 & 41.53 & 24.1 & 24.2 & 25.1 & 27.6 & 31.0 & 34.2 & 37.4 & 40.7 & 43.6 & 45.8 \\
\hline ESF-HD-80-TEMP2-RTD-55 & -9.86 & 2.53 & 3.17 & 42.53 & 24.2 & 24.2 & 25.1 & 27.3 & 30.4 & 33.3 & 36.2 & 39.3 & 42.0 & 44.1 \\
\hline ESF-HD-80-TEMP2-RTD-56 & -9.87 & 3.53 & 3.17 & 43.53 & 24.1 & 24.2 & 24.9 & 26.9 & 29.7 & 32.3 & 34.9 & 37.7 & 40.1 & 42.0 \\
\hline ESF-HD-80-TEMP2-RTD-57 & -9.87 & 4.53 & 3.17 & 4.53 & 24.1 & 24.2 & 24.8 & 26.4 & 28.8 & 31.0 & 33.3 & 35.7 & 37.7 & 39.3 \\
\hline ESF-HD-80-TEMP2-RTD-58 & -9.88 & 5.52 & 3.17 & 45.52 & 24.0 & 24.1 & 24.5 & 25.7 & 27.5 & 29.3 & 31.2 & 33.1 & 34.9 & 36.3 \\
\hline ESF-HD-80-TEMP2-RTD-59 & -9.89 & 6.52 & 3.16 & 46.52 & 24.0 & 24.0 & 24.2 & 25.0 & 26.2 & 27.5 & 28.8 & 30.4 & 31.8 & 32.9 \\
\hline ESF-HD-80-TEMP2-RTD-60 & -9.90 & 7.52 & 3.16 & 7.52 & 23.9 & 23.9 & 1.0 & .4 & 5.2 & 6.1 & 27 & 8.2 & 29.2 & 0.1 \\
\hline \multicolumn{15}{|l|}{ BH 133 Gage ID } \\
\hline ESF-HD-133-TEMP3-RTD-1 & 0.75 & 2.74 & 2.50 & 2.50 & 30.3 & 52.7 & 62.7 & 69.2 & 75.5 & 80.0 & 84.2 & 87.9 & 91.6 & 93.5 \\
\hline ESF-HD-133-TEMP3- & 0.75 & 2.74 & 2.80 & 2.80 & 30.1 & 44.6 & 54.5 & 61.0 & 66.9 & 71.4 & 17.5 & 79.2 & 82.5 & 34.8 \\
\hline ESF-HD-133-TEMP3- & 0.75 & 2.74 & 3.10 & 3.10 & 29.9 & 39.4 & 48.8 & 55.0 & 60.7 & 65.0 & 68.9 & 72.7 & 75.8 & 78.1 \\
\hline ESF-HD- & 0.76 & 2.74 & 3 & 3.40 & 29.8 & 35.5 & 43.8 & 49.6 & 4.9 & 59.0 & $0<.1$ & 6.3 & 69.3 & 1.6 \\
\hline ESF-HD- & 0.76 & 2 & 3 & 3.70 & 29.5 & 32.9 & 40.0 & 45.4 & 0.3 & 4.2 & & 1.2 & 64.1 & 6.3 \\
\hline ESF-HD-1 & 0.76 & .74 & 4.00 & 4.00 & 29.3 & 30.9 & 36.4 & 1.1 & 5.6 & 9.2 & 2.4 & 5.8 & 58.5 & 0.6 \\
\hline ESF-HD & & & & 4.30 & 29.0 & 29.8 & 34.2 & 8.4 & 2.4 & 45.8 & & 2.0 & 54.6 & 6.6 \\
\hline ESF-HD-133-TEMP3 & & & & & & & & & 9.1 & & & 7.8 & 50.3 & \\
\hline ESF-HD-133-TEMP3-RTD-9 & & & & 4.91 & 28.3 & 28.4 & & & 36.8 & 9.6 & & 4.9 & 47.2 & \\
\hline ESF-HD-133-TEMP3-RTD-10 & & & 5.21 & 5.21 & 28.0 & & & & & 36.8 & & 41.5 & 43.6 & \\
\hline ESF-HD-133-TEMP3-RTD-11 & & & & 5.51 & 27.6 & & & & 32.8 & & & 39.2 & 41.2 & \\
\hline ESF-HD-133-TEMP3-RTD-12 & & & & 5.81 & 27.3 & 27.3 & & 29.2 & 31.1 & 32.9 & & 36.8 & 38.6 & \\
\hline ESF-HD-133-TEMP3-RTD-13 & & & & 6.11 & 27.0 & 27.0 & & & 30.0 & 31.6 & & 35.1 & 36.7 & \\
\hline ESF-HD-133-TEMP3-RTD-14 & & & & 6.41 & 26.6 & 26.7 & 26.9 & & 28.5 & 29.9 & & 33.2 & 34.3 & \\
\hline ESF-HD-133-TEMP3-RTD-15 & 0.78 & 2.76 & 6.71 & 6.71 & 26.4 & 26.4 & 26.6 & 27.2 & 28.2 & 29.3 & 30.6 & 32.0 & 33.3 & \\
\hline ESF-HD-133-TEMP3-RTD-16 & 0.78 & 2.76 & 7.01 & 7.01 & 26.0 & 26.1 & 26.2 & 26.6 & 27.4 & 28.3 & 29.4 & 30.6 & 31.7 & \\
\hline ESF-HD-133-TEMP3-RTD-17 & & 2.76 & 7.31 & 7.31 & 25.9 & 25.9 & 26.0 & 26.3 & 26.9 & 27.7 & 28.6 & 29.7 & 30.7 & 31.6 \\
\hline ESF-HD-133-TEMP3-RTD-18 & 0.78 & 2.76 & 7.61 & 7.61 & 25.5 & 25.6 & 25.7 & 25.9 & 26.4 & 27.0 & 27.7 & 28.6 & 29.5 & 30.2 \\
\hline ESF-HD-133-TEMP3-RTD-19 & 0.78 & 2.76 & 7.92 & 7.92 & 25.3 & 25.4 & 25.5 & 25.7 & 26.1 & 26.6 & 27.2 & 28.0 & 28.8 & 29.5 \\
\hline ESF-HD-133-TEMP3-RTD-20 & 0.79 & 2.76 & 8.22 & 8.22 & 25.1 & 25.2 & 25.2 & 25.4 & 25.7 & 26.1 & 26.6 & 27.2 & 27.9 & 28.5 \\
\hline & 0.79 & 2.77 & 8.52 & 8.52 & 24.9 & 25.0 & 25.1 & 25.2 & 25.4 & 25.8 & 26.2 & 26.8 & 27.4 & 27.9 \\
\hline ESF-HD-133-TEMP3-RTD-22 & 0.79 & 2.77 & 8.82 & 8.82 & 24.8 & 24.8 & 24.9 & 25.0 & 25.2 & 25.5 & 25.8 & 26.3 & 26.8 & 27.2 \\
\hline ECE HO 132 TEMP 2 RTD 23 & 0.79 & 2.77 & 9.12 & 9.12 & 24.6 & 24.7 & 24.8 & 24.9 & 25.0 & 25.2 & 25.5 & 25.9 & 26.4 & 26.7 \\
\hline
\end{tabular}


First Quarter Report of Temperature Measurements During the Heating Phase of the Drift-Scale Test

\begin{tabular}{|c|c|c|c|c|c|c|c|c|c|c|c|c|c|c|}
\hline \multirow{5}{*}{\begin{tabular}{|l} 
ESF-HD-133-TEMP3-RTD-24 \\
\end{tabular}} & \multicolumn{14}{|c|}{ Temperature Data Obtained from the DST } \\
\hline & \multicolumn{14}{|c|}{ Days after startup } \\
\hline & \multicolumn{4}{|c|}{ As Built Locations (m) } & 0 & 7 & 17 & 27 & 38 & 48 & 58 & 69 & 79 & 87 \\
\hline & $\mathbf{x}$ & $\mathbf{y}$ & $\mathbf{z}$ & D & \multicolumn{10}{|c|}{$\left({ }^{\circ} \mathbf{C}\right)$} \\
\hline & 0.79 & 2.77 & 9.42 & 9.42 & 24.5 & 24.5 & 24.6 & 24.7 & 24.8 & 25.0 & 25.2 & 25.5 & 25.9 & 26.2 \\
\hline ESF-HD-133-TEMP3-RTD-25 & 0.80 & 2.77 & 9.72 & 9.72 & 24.4 & 24.4 & 24.5 & 24.6 & 24.7 & 24.8 & 25.0 & 25.3 & 25.6 & 25.9 \\
\hline ESF-HD-133-TEMP3-RTD-26 & 0.80 & 2.77 & 10.02 & 10.02 & 24.2 & 24.3 & 24.4 & 24.4 & 24.5 & 24.6 & 24.8 & 25.0 & 25.3 & 25.5 \\
\hline ESF-HD-133-TEMP3-RTD-27 & 0.80 & 2.77 & 10.32 & 10.32 & 22.3 & 24.2 & 24.3 & 24.3 & 24.4 & 24.5 & 24.6 & 24.8 & 25.0 & 25.3 \\
\hline ESF-HD-133-TEMP3-RTD-28 & 0.80 & 2.78 & 10.62 & 10.62 & 24.0 & 24.1 & 24.1 & 24.2 & 24.3 & 24.3 & 24.5 & 24.6 & 24.8 & 25.0 \\
\hline ESF-HD-133-TEMP3-RTD-29 & 0.80 & 2.78 & 10.93 & 10.93 & 24.0 & 24.0 & 24.1 & 24.1 & 24.2 & 24.3 & 24.4 & 24.5 & 24.6 & 24.8 \\
\hline ESF-HD-133-TEMP3-RTD-30 & 0.81 & 2.78 & 11.23 & 11.23 & 23.9 & 23.9 & 24.0 & 24.0 & 24.1 & 24.2 & 24.2 & 24.3 & 24.5 & 24.6 \\
\hline ESF-HD-133-TEMP3-RTD-31 & 0.81 & 2.78 & 11.53 & 11.53 & 23.8 & 23.8 & 23.9 & 23.9 & 24.0 & 24.1 & 24.1 & 24.2 & 24.3 & 24.5 \\
\hline ESF-HD-133-TEMP3-RTD-32 & 0.81 & 2.78 & 11.83 & 11.83 & 23.7 & 23.8 & 23.8 & 23.9 & 23.9 & 24.0 & 24.0 & 24.1 & 24.2 & 24.3 \\
\hline ESF-HD-133-TEMP3-RTD-33 & 0.81 & 2.78 & 12.13 & 12.13 & 23.7 & 23.7 & 23.8 & 23.8 & 23.9 & 23.9 & 24.0 & 24.0 & 24.1 & 24.2 \\
\hline ESF-HD-133-TEMP3-RTD-34 & 0.81 & 2.78 & 12.43 & 12.43 & 23.6 & 23.7 & 23.7 & 23.7 & 23.8 & 23.8 & 23.9 & 23.9 & 24.0 & 24.1 \\
\hline ESF-HD-133-TEMP3-RTD-35 & 0.82 & 2.79 & 12.73 & 12.73 & 23.6 & 23.6 & 23.7 & 23.7 & 23.8 & 23.8 & 23.8 & 23.9 & 24.0 & 24.0 \\
\hline ESF-HD-133-TEMP3-RTD-36 & 0.82 & 2.79 & 13.03 & 13.03 & 23.6 & 23.6 & 23.6 & 23.6 & 23.7 & 23.7 & 23.7 & 23.8 & 23.8 & 23.9 \\
\hline ESF-HD-133-TEMP3-RTD-37 & 0.82 & 2.79 & 13.33 & 13.33 & 23.5 & 23.5 & 23.6 & 23.6 & 23.6 & 23.7 & 23.7 & 23.7 & 23.8 & 23.8 \\
\hline ESF-HD-133-TEMP3-RTD-38 & 0.82 & 2.79 & 13.64 & 13.64 & 23.5 & 23.5 & 23.6 & 23.6 & 23.6 & 23.7 & 23.7 & 23.7 & 23.8 & 23.8 \\
\hline ESF-HD-133-TEMP3-RTD-39 & & 2.79 & & 13.94 & 23.5 & 23.5 & 23.5 & 23.5 & 23.5 & 23.6 & 23.6 & 23.6 & 23.7 & 23.7 \\
\hline ESF-HD-133-TEMP3-RTD-40 & & 2.79 & 14.24 & 14.24 & 23.4 & 23.4 & 23.4 & 23.5 & 23.5 & 23.5 & 23.5 & 23.6 & 23.6 & 23.6 \\
\hline ESF-HD-133-TEMP3-RTD-41 & 0.83 & 2.80 & 14.54 & 14.54 & 23.4 & 23.4 & 23.5 & 23.5 & 23.5 & 23.5 & 23.5 & 23.6 & 23.6 & 23.6 \\
\hline ESF-HD-133-TEMP3-RTD-42 & 0.83 & 2.80 & 14.84 & 14.84 & 23.4 & 23.4 & 23.4 & 23.4 & 23.4 & 23.5 & 23.5 & 23.5 & 23.5 & 23.6 \\
\hline ESF-HD-133-TEMP3-RTD-43 & 0.83 & 2.80 & 15.14 & 15.14 & 23.4 & 23.4 & 23.4 & 23.4 & 23.4 & 23.5 & 23.5 & 23.5 & 23.5 & 23.5 \\
\hline ESF-HD-133-TEMP3-RTD-44 & 0.83 & 2.80 & 15.44 & 15.44 & 23.4 & 23.4 & 23.4 & 23.4 & 23.4 & 23.4 & 23.4 & 23.4 & 23.5 & 23.5 \\
\hline ESF-HD-133-TEMP3-RTD-45 & 0.83 & 2.80 & 15.74 & 15.74 & 23.3 & 23.3 & 23.4 & 23.4 & 23.4 & 23.4 & 23.4 & 23.4 & 23.4 & 23.4 \\
\hline ESF-HD-133-TEMP3-RTD-46 & 0.84 & 2.80 & 16.04 & 16.04 & 23.3 & 23.4 & 23.4 & 23.4 & 23.4 & 23.4 & 23.4 & 23.4 & 23.5 & 23.5 \\
\hline ESF-HD-133-TEMP3-RTD-47 & 0.84 & 2.80 & 16.34 & 16.34 & 23.3 & 23.3 & 23.3 & 23.4 & 23.4 & 23.4 & 23.4 & 23.4 & 23.4 & 23.4 \\
\hline ESF-HD-133-TEMP3-RTD-48 & 0.84 & 2.81 & 16.65 & 16.65 & 23.3 & 23.3 & 23.3 & 23.3 & 23.3 & 23.4 & 23.4 & 23.4 & 23.4 & 23.4 \\
\hline ESF-HD-133-TEMP3-RTD-49 & 0.84 & 2.81 & 16.95 & 16.95 & 23.3 & 23.3 & 23.3 & 23.3 & 23.3 & 23.3 & 23.3 & 23.4 & 23.4 & 23.4 \\
\hline ESF-HD-133-TEMP3-RTD-50 & 0.84 & 2.81 & 17.25 & 17.25 & 23.3 & 23.3 & 23.3 & 23.3 & 23.3 & 23.3 & 23.3 & 23.3 & 23.3 & 23.3 \\
\hline ESF-HD-133-TEMP3-RTD-51 & 0.85 & 2.81 & 17.55 & 17.55 & 23.3 & 23.3 & 23.3 & 23.3 & 23.3 & 23.3 & 23.3 & 23.3 & 23.3 & 23.3 \\
\hline ESF-HD-133-TEMP3-RTD-52 & 0.85 & 2.81 & 17.85 & 17.85 & 23.3 & 23.2 & 23.3 & 23.3 & 23.3 & 23.3 & 23.3 & 23.3 & 23.3 & 23.3 \\
\hline ESF-HD-133-TEMP3-RTD-53 & 0.85 & 2.81 & 18.15 & 18.15 & 23.3 & 23.3 & 23.3 & 23.3 & 23.3 & 23.3 & 23.3 & 23.3 & 23.3 & 23.3 \\
\hline ESF-HD-133-TEMP3-RTD-54 & 0.85 & 2.81 & 18.45 & 18.45 & 23.2 & 23.2 & 23.2 & 23.2 & 23.3 & 23.3 & 23.3 & 23.3 & 23.3 & 23.3 \\
\hline ESF-HD-133-TEMP3-RTD-55 & 0.85 & 2.82 & 18.75 & 18.75 & 23.2 & 23.2 & 23.2 & 23.2 & 23.2 & 23.3 & 23.3 & 23.3 & 23.3 & 23.3 \\
\hline ESF-HD-133-TEMP3-RTD-56 & 0.86 & 2.82 & 19.05 & 19.05 & 21.6 & 21.7 & 21.8 & 21.9 & 21.9 & 22.0 & 22.0 & 22.0 & 22.0 & 22.1 \\
\hline ESF-HD-133-TEMP3-RTD-57 & 0.86 & 2.82 & 19.35 & 19.35 & 23.2 & 23.2 & 23.2 & 23.2 & 23.2 & 23.2 & 23.2 & 23.2 & 23.2 & 23.2 \\
\hline ESF-HD-133-TEMP3-RTD-58 & 0.86 & 2.82 & 19.66 & 19.66 & 23.2 & 23.2 & 23.2 & 23.2 & 23.2 & 23.2 & 23.2 & 23.2 & 23.2 & 23.2 \\
\hline ESF-HD-133-TEMP3-RTD-59 & 0.86 & 2.82 & 19.96 & 19.96 & 23.2 & 23.2 & 23.2 & 23.2 & 23.2 & 23.2 & 23.2 & 23.2 & 23.2 & 23.2 \\
\hline ESF-HD-133-TEMP3-RTD-60 & 0.86 & 2.82 & 20.26 & 20.26 & 23.2 & 23.2 & 23.2 & 23.2 & 23.2 & 23.2 & 23.2 & 23.2 & 23.2 & 23.2 \\
\hline
\end{tabular}




\begin{tabular}{|c|c|c|c|c|c|c|c|c|c|c|c|c|c|c|}
\hline & \multicolumn{14}{|c|}{ Temperature Data Obtained from the DST } \\
\hline & \multicolumn{14}{|c|}{ Days after startup } \\
\hline & \multicolumn{4}{|c|}{ As Built Locations (m) } & 0 & 7 & 17 & 27 & 38 & 48 & 58 & 69 & 79 & 87 \\
\hline & $\mathbf{x}$ & $\mathbf{y}$ & $\mathbf{z}$ & D & \multicolumn{10}{|c|}{$\left({ }^{\circ} \mathbf{C}\right)$} \\
\hline ESF-HD-133-TEMP3-RTD-61 & 0.87 & 2.82 & 20.56 & 20.56 & 23.1 & 23.1 & 23.1 & 23.1 & 23.1 & 23.1 & 23.1 & 23.1 & 23.1 & 23.1 \\
\hline ESF-HD-133-TEMP3-RTD-62 & 0.87 & 2.83 & 20.86 & 20.86 & 23.2 & 23.2 & 23.2 & 23.2 & 23.2 & 23.2 & 23.2 & 23.2 & 23.2 & 23.2 \\
\hline ESF-HD-133-TEMP3-RTD-63 & 0.87 & 2.83 & 21.16 & 21.16 & 23.2 & 23.2 & 23.2 & 23.2 & 23.2 & 23.2 & 23.2 & 23.2 & 23.2 & 23.2 \\
\hline ESF-HD-133-TEMP3-RTD-64 & 0.87 & 2.83 & 21.46 & 21.46 & 23.2 & 23.2 & 23.7 & 23.2 & 23.2 & 23.2 & 23.2 & 23.2 & 23.2 & 23.2 \\
\hline ESF-HD-133-TEMP3-RTD-65 & 0.87 & 2.83 & 21.76 & 21.76 & 23.2 & 23.2 & 23.2 & 23.2 & 23.2 & 23.2 & 23.2 & 23.2 & 23.2 & 23.2 \\
\hline ESF-HD-133-TEMP3-RTD-66 & 0.88 & 2.83 & 22.06 & 22.06 & 23.2 & 23.2 & 23.2 & 23.2 & 23.2 & 23.2 & 23.2 & 23.2 & 23.2 & 23.2 \\
\hline ESF-HD-133-TEMP3-RTD-67 & 0.88 & 2.83 & 22.36 & 22.36 & 23.2 & 23.2 & 23.2 & 23.2 & 23.2 & 23.2 & 23.2 & 23.2 & 23.2 & 23.2 \\
\hline \multicolumn{15}{|l|}{ BH 134 Gage ID } \\
\hline ESF-HD-134-TEMP4-RTD-1 & 0.74 & 2.73 & -0.92 & 0.92 & 30.0 & 40.0 & 48.9 & 55.0 & 60.4 & 64.5 & 68.2 & 71.8 & 74.7 & 76.8 \\
\hline ESF-HD-134-TEMP4-RTD-2 & 0.74 & 2.73 & -1.23 & 1.23 & 29.9 & 36.3 & 44.5 & 50.2 & 55.5 & 59.5 & 63.0 & 66.5 & 69.5 & 71.5 \\
\hline ESF-HD-134-TEMP4-RTD-3 & 0.74 & 2.73 & -1.55 & 1.55 & 29.8 & 33.3 & 40.4 & 45.8 & 50.6 & 54.3 & 57.7 & 61.1 & 63.9 & 65.9 \\
\hline ESF-HD-134-TEMP4-RTD-4 & 0.73 & 2.73 & -1.87 & 1.87 & 29.6 & 31.7 & 37.7 & 42.6 & 47.1 & 50.7 & 54.0 & 57.3 & 59.9 & 61.9 \\
\hline ESF-HD-134-TEMP4-RTD-5 & 0.73 & 2.74 & -2.18 & 2.18 & 29.2 & 30.0 & 34.4 & 38.6 & 42.6 & 45.9 & 49.0 & 52.0 & 54.6 & 56.5 \\
\hline ESF-HD-134-TEMP4-RTD-6 & 0.73 & 2.74 & -2.50 & 2.50 & 28.9 & 29.3 & 32.6 & 36.2 & 39.8 & 42.9 & 45.7 & 48.6 & 51.0 & 52.8 \\
\hline ESF-HD-134-TEMP4-RTD-7 & 0.73 & 2.74 & -2.81 & 2.81 & 28.5 & 28.6 & 30.8 & 33.8 & 36.9 & 39.6 & 42.2 & 44.8 & 47.1 & 48.8 \\
\hline ESF-HD-134-TEMP4-RTD-8 & 0.73 & 2.74 & -3.13 & 3.13 & 28.2 & 28.2 & 29.8 & 32.2 & 35.0 & 37.5 & 39.8 & 42.3 & 44.5 & 46.1 \\
\hline ESF-HD-134-TEMP4-RTD-9 & 0.73 & 2.74 & -3.44 & 3.44 & 27.8 & 27.7 & 28.7 & 30.6 & 32.9 & 35.0 & 37.1 & 39.4 & 41.3 & 42.9 \\
\hline ESF-HD-134-TEMP4-RTD-10 & 0.73 & 2.74 & -3.76 & 3.76 & 27.5 & 27.5 & 28.1 & 29.6 & 31.5 & 33.4 & 35.3 & 37.4 & 39.2 & 40.7 \\
\hline ESF-HD-134-TEMP4-RTD-11 & 0.72 & 2.74 & -4.08 & 4.08 & 27.1 & 27.1 & 27.5 & 28.5 & 30.1 & 31.7 & 33.3 & 35.2 & 36.8 & 38.1 \\
\hline ESF-HD-134-TEMP4-RTD-12 & 0.72 & 2.74 & -4.39 & 4.39 & 26.8 & 26.8 & 27.1 & 27.9 & 29.2 & 30.6 & 32.0 & 33.7 & 35.2 & 36.4 \\
\hline ESF-HD-134-TEMP4-RTD-13 & 0.72 & 2.74 & -4.71 & 4.71 & 26.5 & 26.5 & 26.7 & 27.3 & 28.3 & 29.4 & 30.7 & 32.1 & 33.5 & 34.6 \\
\hline ESF-HD-134-TEMP4-RTD-14 & 0.72 & 2.74 & -5.02 & 5.02 & 26.3 & 26.3 & 26.4 & 26.9 & 27.7 & 28.7 & 29.8 & 31.1 & 32.3 & 33.3 \\
\hline ESF-HD-134-TEMP4-RTD-15 & 0.72 & 2.74 & -5.34 & 5.34 & 26.0 & 26.0 & 26.1 & 26.4 & 27.0 & 27.8 & 28.7 & 29.9 & 30.9 & 31.8 \\
\hline ESF-HD-134-TEMP4-RTD-16 & 0.72 & 2.74 & -5.65 & 5.65 & 25.8 & 25.8 & 25.9 & 26.1 & 26.6 & 27.3 & 28.1 & 29.1 & 30.0 & 30.8 \\
\hline ESF-HD-134-TEMP4-RTD-17 & 0.72 & 2.74 & -5.97 & 5.97 & 25.5 & 25.6 & 25.7 & 25.8 & 26.2 & 26.7 & 27.4 & 28.2 & 29.1 & 29.8 \\
\hline ESF-HD-134-TEMP4-RTD-18 & 0.71 & 2.75 & -6.29 & 6.29 & 25.4 & 25.4 & 25.5 & 25.7 & 26.0 & 26.4 & 27.0 & 27.7 & 28.4 & 29.1 \\
\hline ESF-HD-134-TEMP4-RTD-19 & 0.71 & 2.75 & -6.60 & 6.60 & 25.2 & 25.2 & 25.3 & 25.4 & 25.7 & 26.0 & 26.5 & 27.1 & 27.7 & 28.3 \\
\hline ESF-HD-134-TEMP4-RTD-20 & 0.71 & 2.75 & -6.92 & 6.92 & 25.0 & 25.1 & 25.1 & 25.2 & 25.4 & 25.7 & 26.1 & 26.6 & 27.1 & 27.6 \\
\hline ESF-HD-134-TEMP4-RTD-21 & 0.71 & 2.75 & -7.23 & 7.23 & 24.9 & 24.9 & 25.0 & 25.1 & 25.2 & 25.5 & 25.8 & 26.2 & 26.7 & 27.1 \\
\hline ESF-HD-134-TEMP4-RTD-22 & 0.71 & 2.75 & -7.55 & 7.55 & 24.7 & 24.8 & 24.9 & 25.0 & 25.1 & 25.3 & 25.5 & 25.9 & 26.3 & 26.7 \\
\hline ESF-HD-134-TEMP4-RTD-23 & 0.71 & 2.75 & -7.86 & 7.86 & 24.6 & 24.6 & 24.7 & 24.8 & 24.9 & 25.0 & 25.3 & 25.6 & 25.9 & 26.2 \\
\hline ESF-HD-134-TEMP4-RTD-24 & 0.70 & 2.75 & -8.18 & 8.18 & 24.5 & 24.5 & 24.6 & 24.7 & 24.8 & 24.9 & 25.1 & 25.3 & 25.6 & 25.9 \\
\hline ESF-HD-134-TEMP4-RTD-25 & 0.70 & 2.75 & -8.50 & 8.50 & 24.4 & 24.4 & 24.5 & 24.6 & 24.6 & 24.7 & 24.9 & 25.1 & 25.3 & 25.6 \\
\hline ESF-HD-134-TEMP4-RTD-26 & 0.70 & 2.75 & -8.81 & 8.81 & 24.3 & 24.3 & 24.4 & 24.5 & 24.6 & 24.6 & 24.8 & 24.9 & 25.1 & 25.3 \\
\hline ESF-HD-134-TEMP4-RTD-27 & 0.70 & 2.75 & -9.13 & 9.13 & 24.2 & 24.2 & 24.3 & 24.4 & 24.4 & 24.5 & 24.6 & 24.7 & 24.9 & 25.1 \\
\hline ESF-HD-134-TEMP4-RTD-28 & 0.70 & 2.75 & -9.44 & 9.44 & 24.1 & 24.2 & 24.2 & 24.3 & 24.4 & 24.4 & 24.5 & 24.6 & 24.8 & 24.9 \\
\hline ESF-HD-134-TEMP4-RTD-29 & 0.70 & 2.75 & -9.76 & 9.76 & 24.1 & 24.1 & 24.2 & 24.2 & 24.3 & 24.3 & 24.4 & 24.5 & 24.6 & 24.7 \\
\hline
\end{tabular}


First Quarter Report of Temperature Measurements During the Heating Phase of the Drift-Scale Test

\begin{tabular}{|c|c|c|c|c|c|c|c|c|c|c|c|c|c|c|}
\hline \multirow{5}{*}{\begin{tabular}{|l} 
ESF-HD-134-TEMP4-RTD-30 \\
\end{tabular}} & \multicolumn{14}{|c|}{ Temperature Data Obtained from the DST } \\
\hline & \multicolumn{14}{|c|}{ Days after startup } \\
\hline & \multicolumn{4}{|c|}{ As Built Locations (m) } & 0 & 7 & 17 & 27 & 38 & 48 & 58 & 69 & 79 & 87 \\
\hline & $\mathbf{x}$ & $\mathbf{y}$ & $\mathbf{z}$ & $\mathbf{D}$ & \multicolumn{10}{|c|}{$\left({ }^{\circ} \mathrm{C}\right)$} \\
\hline & 0.70 & 2.75 & -10.07 & 10.07 & 24.0 & 24.1 & 24.1 & 24.2 & 24.2 & 24.3 & 24.3 & 24.4 & 24.5 & 24.6 \\
\hline ESF-HD-134-TEMP4-RTD-31 & 0.69 & 2.76 & -10.39 & 10.39 & 24.0 & 24.0 & 24.0 & 24.1 & 24.1 & 24.2 & 24.2 & 24.3 & 24.4 & 24.5 \\
\hline ESF-HD-134-TEMP4-RTD-32 & 0.69 & 2.76 & -10.71 & 10.71 & 23.9 & 24.0 & 24.0 & 24.0 & 24.1 & 24.1 & 24.2 & 24.2 & 24.3 & 24.4 \\
\hline ESF-HD-134-TEMP4-RTD-33 & 0.69 & 2.76 & -11.02 & 11.02 & 23.9 & 23.9 & 24.0 & 24.0 & 24.0 & 24.1 & 24.1 & 24.2 & 24.2 & 24.3 \\
\hline ESF-HD-134-TEMP4-RTD-34 & 0.69 & 2.76 & -11.34 & 11.34 & 23.9 & 23.9 & 24.0 & 24.0 & 24.0 & 24.1 & 24.1 & 24.2 & 24.2 & 24.3 \\
\hline ESF-HD-134-TEMP4-RTD-35 & 0.69 & 2.76 & -11.65 & 11.65 & 23.9 & 23.9 & 23.9 & 23.9 & 23.9 & 24.0 & 24.0 & 24.0 & 24.1 & 24.1 \\
\hline ESF-HD-134-TEMP4-RTD-36 & 0.69 & 2.76 & -11.97 & 11.97 & 23.8 & 23.8 & 23.9 & 23.9 & 23.9 & 24.0 & 24.0 & 24.0 & 24.1 & 24.1 \\
\hline ESF-HD-134-TEMP4-RTD-37 & 0.69 & 2.76 & -12.28 & 12.28 & 23.8 & 23.8 & 23.8 & 23.9 & 23.9 & 23.9 & 23.9 & 24.0 & 24.0 & 24.0 \\
\hline ESF-HD-134-TEMP4-RTD-38 & 0.68 & 2.76 & -12.60 & 12.60 & 23.6 & 23.6 & 23.6 & 23.6 & 23.7 & 23.7 & 23.8 & 23.8 & 23.9 & 23.9 \\
\hline ESF-HD-134-TEMP4-RTD-39 & 0.68 & 2.76 & -12.92 & 12.92 & 23.8 & 23.8 & 23.8 & 23.9 & 23.9 & 23.9 & 23.9 & 23.9 & 24.0 & 24.0 \\
\hline ESF-HD-134-TEMP4-RTD-40 & 0.68 & 2.76 & -13.23 & 13.23 & 23.8 & 23.8 & 23.8 & 23.8 & 23.9 & 23.9 & 23.9 & 23.9 & 23.9 & 24.0 \\
\hline ESF-HD-134-TEMP4-RTD-41 & 0.68 & 2.76 & -13.55 & 13.55 & 23.8 & 23.8 & 23.8 & 23.8 & 23.8 & 23.8 & 23.8 & 23.8 & 23.9 & 23.9 \\
\hline ESF-HD-134-TEMP4-RTD-42 & 0.68 & 2.76 & -13.86 & 13.86 & 23.8 & 23.8 & 23.8 & 23.8 & 23.9 & 23.9 & 23.9 & 23.9 & 23.9 & 23.9 \\
\hline ESF-HD-134-TEMP4-RTD-43 & 0.68 & 2.77 & -14.18 & 14.18 & 24.1 & 24.1 & 24.1 & 24.2 & 24.2 & 24.2 & 24.2 & 24.3 & 24.3 & 24.3 \\
\hline ESF-HD-134-TEMP4-RTD-44 & 0.67 & 2.77 & -14.49 & 14.49 & 23.8 & 23.8 & 23.8 & 23.8 & 23.8 & 23.8 & 23.8 & 23.8 & 23.8 & 23.9 \\
\hline ESF-HD-134-TEMP4-RTD-45 & 0.67 & 2.77 & -14.81 & 14.81 & 23.7 & 23.7 & 23.7 & 23.7 & 23.7 & 23.8 & 23.8 & 23.8 & 23.8 & 23.8 \\
\hline ESF-HD-134-TEMP4-RTD-46 & 0.67 & 2.77 & -15.13 & 15.13 & 23.8 & 23.8 & 23.8 & 23.8 & 23.8 & 23.8 & 23.8 & 23.8 & 23.8 & 23.8 \\
\hline & & 2.77 & -15.44 & & 23.8 & & & & & & & & 23.8 & 23.8 \\
\hline & & 2.77 & -15.76 & 15.76 & 23.8 & & & & 23.8 & & & 23.8 & 23.8 & 23.8 \\
\hline ESF-HD-134-TEMP4-RTD-49 & 0.67 & 2.77 & -16.07 & 16.07 & 23.8 & 23.8 & 23.8 & 23.8 & 23.8 & 23.8 & 23.8 & 23.8 & 23.8 & 23.8 \\
\hline ESF-HD-134-TEMP4-RTD-50 & 0.67 & 2.77 & -16.39 & 16.39 & 23.8 & 23.8 & 23.8 & 23.8 & 23.8 & 23.8 & 23.8 & 23.8 & 23.8 & 23.8 \\
\hline ESF-HD-134-TEMP4-RTD-51 & 0.66 & 2.77 & -16.70 & 16.70 & 23.8 & 23.8 & 23.8 & 23.8 & 23.8 & 23.8 & 23.8 & 23.8 & 23.8 & 23.8 \\
\hline ESF-HD-134-TEMP4-RTD-52 & 0.66 & 2.77 & -17.02 & 17.02 & 23.9 & 23.9 & 23.9 & 23.9 & 23.9 & 23.9 & 24.0 & 24.0 & 24.0 & 24.0 \\
\hline ESF-HD-134-TEMP4-RTD-53 & 0.66 & 2.77 & -17.34 & 17.34 & 23.8 & 23.8 & 23.8 & 23.8 & 23.8 & 23.8 & 23.8 & 23.8 & 23.8 & 23.8 \\
\hline ESF-HD-134-TEMP4-RTD-54 & 0.66 & 2.77 & -17.65 & 17.65 & 23.8 & 23.8 & 23.8 & 23.8 & 23.8 & 23.8 & 23.8 & 23.8 & 23.8 & 23.8 \\
\hline ESF-HD-134-TEMP4-RTD-55 & 0.66 & 2.77 & -17.97 & 17.97 & 23.8 & 23.8 & 23.8 & 23.8 & 23.8 & 23.8 & 23.8 & 23.8 & 23.8 & 23.8 \\
\hline ESF-HD-134-TEMP4-RTD-56 & 0.66 & 2.78 & -18.28 & 18.28 & 23.8 & 23.8 & 23.8 & 23.8 & 23.8 & 23.8 & 23.8 & 23.8 & 23.8 & 23.8 \\
\hline ESF-HD-134-TEMP4-RTD-57 & 0.66 & 2.78 & -18.60 & 18.60 & 23.8 & 23.8 & 23.8 & 23.8 & 23.8 & 23.8 & 23.8 & 23.8 & 23.8 & 23.8 \\
\hline ESF-HD-134-TEMP4-RTD-58 & 0.65 & 2.78 & -18.91 & 18.91 & 23.7 & 23.8 & 23.8 & 23.8 & 23.8 & 23.8 & 23.8 & 23.8 & 23.8 & 23.8 \\
\hline ESF-HD-134-TEMP4-RTD-59 & 0.65 & 2.78 & -19.23 & 19.23 & 23.8 & 23.8 & 23.8 & 23.8 & 23.8 & 23.8 & 23.8 & 23.8 & 23.8 & 23.8 \\
\hline ESF-HD-134-TEMP4-RTD-60 & 0.65 & 2.78 & -19.54 & 19.54 & 23.8 & 23.8 & 23.8 & 23.8 & 23.8 & 23.8 & 23.8 & 23.8 & 23.8 & 23.8 \\
\hline ESF-HD-134-TEMP4-RTD-61 & 0.65 & 2.78 & -19.86 & 19.86 & 23.8 & 23.8 & 23.8 & 23.8 & 23.8 & 23.8 & 23.8 & 23.8 & 23.8 & 23.8 \\
\hline ESF-HD-134-TEMP4-RTD-62 & 0.65 & 2.78 & -20.18 & 20.18 & 23.8 & 23.8 & 23.8 & 23.8 & 23.8 & 23.8 & 23.8 & 23.8 & 23.8 & 23.8 \\
\hline ESF-HD-134-TEMP4-RTD-63 & 0.65 & 2.78 & -20.49 & 20.49 & 23.8 & 23.8 & 23.8 & 23.8 & 23.8 & 23.8 & 23.8 & 23.8 & 23.8 & 23.8 \\
\hline ESF-HD-134-TEMP4-RTD-64 & 0.65 & 2.78 & -20.81 & 20.81 & 23.8 & 23.8 & 23.8 & 23.8 & 23.8 & 23.8 & 23.8 & 23.8 & 23.8 & 23.8 \\
\hline ESF-HD-134-TEMP4-RTD-65 & 0.64 & 2.78 & -21.12 & 21.12 & 23.8 & 23.8 & 23.8 & 23.8 & 23.8 & 23.8 & 23.8 & 23.8 & 23.8 & 23.8 \\
\hline
\end{tabular}




\begin{tabular}{|c|c|c|c|c|c|c|c|c|c|c|c|c|c|c|}
\hline & \multicolumn{14}{|c|}{ Temperature Data Obtained from the DST } \\
\hline & \multicolumn{14}{|c|}{ Days after startup } \\
\hline & \multicolumn{4}{|c|}{ As Built Locations (m) } & 0 & 7 & 17 & 27 & 38 & 48 & 58 & 69 & 79 & 87 \\
\hline & $\mathbf{x}$ & $\mathbf{y}$ & $\mathbf{z}$ & D & \multicolumn{10}{|c|}{$\left({ }^{\circ} \mathbf{C}\right)$} \\
\hline ESF-HD-134-TEMP4-RTD-66 & 0.64 & 2.78 & -21.44 & 21.44 & 24.6 & 24.6 & 24.6 & 24.6 & 24.6 & 24.6 & 24.6 & 24.6 & 24.6 & 24.6 \\
\hline ESF-HD-134-TEMP4-RTD-67 & 0.64 & 2.78 & -21.76 & 21.76 & 23.8 & 23.8 & 23.8 & 23.8 & 23.8 & 23.8 & 23.8 & 23.8 & 23.8 & 23.8 \\
\hline \multicolumn{15}{|l|}{ BH 137 Gage ID } \\
\hline ESF-HD-137-TEMP5-RTD-1 & 0.77 & 11.92 & 2.31 & 2.31 & 28.7 & 63.1 & 72.9 & 79.8 & 86.4 & 91.1 & 95.5 & 99.1 & 103.3 & 105.2 \\
\hline ESF-HD-137-TEMP5-RTD-2 & 0.78 & 11.92 & 2.61 & 2.61 & 29.9 & 52.3 & 62.7 & 69.7 & 76.1 & 81.0 & 85.3 & 89.2 & 92.8 & 94.9 \\
\hline ESF-HD-137-TEMP5-RTD-3 & 0.78 & 11.92 & 2.91 & 2.91 & 29.9 & 45.3 & 55.4 & 62.2 & 68.4 & 73.2 & 77.5 & 81.5 & 84.9 & 87.3 \\
\hline ESF-HD-137-TEMP5-RTD-4 & 0.78 & 11.92 & 3.21 & 3.21 & 29.8 & 39.0 & 48.3 & 54.8 & 60.6 & 65.2 & 69.4 & 73.4 & 76.7 & 79.2 \\
\hline ESF-HD-137-TEMP5-RTD-5 & 0.78 & 11.91 & 3.51 & 3.51 & 29.6 & 35.4 & 43.7 & 49.8 & 55.3 & 59.8 & 63.7 & 67.7 & 71.0 & 73.5 \\
\hline ESF-HD-137-TEMP5-RTD-6 & 0.78 & 11.91 & 3.81 & 3.81 & 29.4 & 32.5 & 39.4 & 44.9 & 50.1 & 54.2 & 58.0 & 61.9 & 65.1 & 67.5 \\
\hline ESF-HD-137-TEMP5-RTD-7 & 0.78 & 11.91 & 4.11 & 4.11 & 29.2 & 30.8 & 36.3 & 41.3 & 46.0 & 49.9 & 53.6 & 57.3 & 60.4 & 62.7 \\
\hline ESF-HD-137-TEMP5-RTD-8 & 0.79 & 11.91 & 4.41 & 4.41 & 28.8 & 29.5 & 33.6 & 37.9 & 42.1 & 45.7 & 49.1 & 52.6 & 55.6 & 57.9 \\
\hline ESF-HD-137-TEMP5-RTD-9 & 0.79 & 11.91 & 4.71 & 4.71 & 28.5 & 28.7 & 31.7 & 35.3 & 39.0 & 42.3 & 45.4 & 48.7 & 51.6 & 53.8 \\
\hline ESF-HD-137-TEMP5-RTD-10 & 0.79 & 11.91 & 5.00 & 5.00 & 28.2 & 28.2 & 30.2 & 33.1 & 36.3 & 39.2 & 42.1 & 45.1 & 47.8 & 49.9 \\
\hline ESF-HD-137-TEMP5-RTD-11 & 0.79 & 11.91 & 5.30 & 5.30 & 27.9 & 27.8 & 29.2 & 31.5 & 34.4 & 37.0 & 39.6 & 42.3 & 44.9 & 46.8 \\
\hline ESF-HD-137-TEMP5-RTD-12 & 0.79 & 11.91 & 5.60 & 5.60 & 27.5 & 27.4 & 28.3 & 30.1 & 32.4 & 34.7 & 37.0 & 39.5 & 41.8 & 43.6 \\
\hline ESF-HD-137-TEMP5-RTD-13 & 0.79 & 11.91 & 5.90 & 5.90 & 27.1 & 27.1 & 27.7 & 29.1 & 31.0 & 33.0 & 35.1 & 37.4 & 39.5 & 41.1 \\
\hline ESF-HD-137-TEMP5-RTD-14 & 0.79 & 11.90 & 6.20 & 6.20 & 26.8 & 26.8 & 27.1 & 28.2 & 29.7 & 31.4 & 33.2 & 35.3 & 37.2 & 38.8 \\
\hline ESF-HD-137-TEMP5-RTD-15 & 0.80 & 11.90 & 6.50 & 6.50 & 26.5 & 26.5 & 26.7 & 27.5 & 28.8 & 30.2 & 31.8 & 33.6 & 35.3 & 36.7 \\
\hline ESF-HD-137-TEMP5-RTD-16 & 0.80 & 11.90 & 6.80 & 6.80 & 26.1 & 26.1 & 26.4 & 27.0 & 28.0 & 29.2 & 30.5 & 32.2 & 33.7 & 35.0 \\
\hline ESF-HD-137-TEMP5-RTD-17 & 0.80 & 11.90 & 7.10 & 7.10 & 26.0 & 26.0 & 26.1 & 26.5 & 27.3 & 28.3 & 29.5 & 30.9 & 32.3 & 33.4 \\
\hline ESF-HD-137-TEMP5-RTD-18 & 0.80 & 11.90 & 7.40 & 7.40 & 25.7 & 25.8 & 25.9 & 26.1 & 26.8 & 27.6 & 28.6 & 29.8 & 31.0 & 32.0 \\
\hline ESF-HD-137-TEMP5-RTD-19 & 0.80 & 11.90 & 7.70 & 7.70 & 25.5 & 25.6 & 25.6 & 25.8 & 26.3 & 27.0 & 27.8 & 28.9 & 29.9 & 30.8 \\
\hline ESF-HD-137-TEMP5-RTD-20 & 0.80 & 11.90 & 8.00 & 8.00 & 25.3 & 25.4 & 25.4 & 25.6 & 26.0 & 26.5 & 27.2 & 28.1 & 29.0 & 29.8 \\
\hline ESF-HD-137-TEMP5-RTD-21 & 0.81 & 11.90 & 8.30 & 8.30 & 25.1 & 25.1 & 25.2 & 25.3 & 25.6 & 26.0 & 26.6 & 27.3 & 28.1 & 28.8 \\
\hline ESF-HD-137-TEMP5-RTD-22 & 0.81 & 11.89 & 8.60 & 8.60 & 24.9 & 25.0 & 25.0 & 25.2 & 25.4 & 25.7 & 26.2 & 26.8 & 27.5 & 28.1 \\
\hline ESF-HD-137-TEMP5-RTD-23 & 0.81 & 11.89 & 8.90 & 8.90 & 24.7 & 24.8 & 24.9 & 25.0 & 25.1 & 25.4 & 25.8 & 26.3 & 26.9 & 27.4 \\
\hline ESF-HD-137-TEMP5-RTD-24 & 0.81 & 11.89 & 9.20 & 9.20 & 24.6 & 24.6 & 24.7 & 24.8 & 25.0 & 25.2 & 25.5 & 25.9 & 26.4 & 26.9 \\
\hline ESF-HD-137-TEMP5-RTD-25 & 0.81 & 11.89 & 9.50 & 9.50 & 24.5 & 24.5 & 24.6 & 24.7 & 24.8 & 24.9 & 25.2 & 25.6 & 26.0 & 26.4 \\
\hline ESF-HD-137-TEMP5-RTD-26 & 0.81 & 11.89 & 9.79 & 9.79 & 24.3 & 24.3 & 24.4 & 24.5 & 24.6 & 24.7 & 24.9 & 25.2 & 25.6 & 25.9 \\
\hline ESF-HD-137-TEMP5-RTD-27 & 0.82 & 11.89 & 10.09 & 10.09 & 24.2 & 24.2 & 24.3 & 24.4 & 24.5 & 24.6 & 24.7 & 25.0 & 25.3 & 25.6 \\
\hline ESF-HD-137-TEMP5-RTD-28 & 0.82 & 11.89 & 10.39 & 10.39 & 24.1 & 24.2 & 24.2 & 24.3 & 24.4 & 24.5 & 24.6 & 24.8 & 25.0 & 25.3 \\
\hline ESF-HD-137-TEMP5-RTD-29 & 0.82 & 11.89 & 10.69 & 10.69 & 24.0 & 24.1 & 24.2 & 24.2 & 24.3 & 24.4 & 24.5 & 24.6 & 24.9 & 25.1 \\
\hline ESF-HD-137-TEMP5-RTD-30 & 0.82 & 11.89 & 10.99 & 10.99 & 24.0 & 24.0 & 24.1 & 24.1 & 24.2 & 24.3 & 24.4 & 24.5 & 24.7 & 24.8 \\
\hline ESF-HD-137-TEMP5-RTD-31 & 0.82 & 11.88 & 11.29 & 11.29 & 23.9 & 23.9 & 24.0 & 24.0 & 24.1 & 24.2 & 24.2 & 24.4 & 24.5 & 24.6 \\
\hline ESF-HD-137-TEMP5-RTD-32 & 0.82 & 11.88 & 11.59 & 11.59 & 23.8 & 23.8 & 23.8 & 23.9 & 23.9 & 24.0 & 24.1 & 24.2 & 24.3 & 24.4 \\
\hline ESF-HD-137-TEMP5-RTD-33 & 0.82 & 11.88 & 11.89 & 11.89 & 23.8 & 23.8 & 23.9 & 23.9 & 23.9 & 24.0 & 24.0 & 24.1 & 24.2 & 24.3 \\
\hline ESF-HD-137-TEMP5-RTD-34 & 0.83 & 11.88 & 12.19 & 12.19 & 23.5 & 23.4 & 23.7 & 23.7 & 23.8 & 23.8 & 23.9 & 23.9 & 24.0 & 24.1 \\
\hline
\end{tabular}


First Quarter Report of Temperature Measurements During the Heating Phase of the Drift-Scale Test

\begin{tabular}{|c|c|c|c|c|c|c|c|c|c|c|c|c|c|c|}
\hline & \multicolumn{14}{|c|}{ Temperature Data Obtained from the DST } \\
\hline & \multicolumn{14}{|c|}{ Days after startup } \\
\hline & \multicolumn{4}{|c|}{ As Built Locations (m) } & 0 & 7 & 17 & 27 & 38 & 48 & 58 & 69 & 79 & 87 \\
\hline & $\mathbf{x}$ & $\mathbf{y}$ & $\mathbf{z}$ & D & \multicolumn{10}{|c|}{$\left({ }^{\circ} \mathbf{C}\right)$} \\
\hline ESF-HD-137-TEMP5-RTD-35 & 0.83 & 11.88 & 12.49 & 12.49 & 23.6 & 23.7 & 23.7 & 23.7 & 23.8 & 23.8 & 23.8 & 23.9 & 24.0 & 24.0 \\
\hline ESF-HD-137-TEMP5-RTD-36 & 0.83 & 11.88 & 12.79 & 12.79 & 23.6 & 23.6 & 23.6 & 23.7 & 23.7 & 23.7 & 23.8 & 23.8 & 23.9 & 24.0 \\
\hline ESF-HD-137-TEMP5-RTD-37 & 0.83 & 11.88 & 13.09 & 13.09 & 23.6 & 23.6 & 23.7 & 23.7 & 23.7 & 23.7 & 23.8 & 23.8 & 23.9 & 23.9 \\
\hline ESF-HD-137-TEMP5-RTD-38 & 0.83 & 11.88 & 13.39 & 13.39 & 23.5 & 23.6 & 23.6 & 23.6 & 23.6 & 23.7 & 23.7 & 23.7 & 23.8 & 23.8 \\
\hline ESF-HD-137-TEMP5-RTD-39 & 0.83 & 11.87 & 13.69 & 13.69 & 23.3 & 23.4 & 23.5 & 23.6 & 23.6 & 23.6 & 23.7 & 23.7 & 23.7 & 23.8 \\
\hline ESF-HD-137-TEMP5-RTD-40 & 0.84 & 11.87 & 13.99 & 13.99 & 23.5 & 23.5 & 23.5 & 23.5 & 23.6 & 23.6 & 23.6 & 23.6 & 23.7 & 23.7 \\
\hline ESF-HD-137-TEMP5-RTD-41 & 0.84 & 11.87 & 14.28 & 14.28 & 23.5 & 23.5 & 23.5 & 23.5 & 23.5 & 23.6 & 23.6 & 23.6 & 23.6 & 23.7 \\
\hline ESF-HD-137-TEMP5-RTD-42 & 0.84 & 11.87 & 14.58 & 14.58 & 23.4 & 23.4 & 23.5 & 23.5 & 23.5 & 23.5 & 23.5 & 23.5 & 23.6 & 23.6 \\
\hline ESF-HD-137-TEMP5-RTD-43 & 0.84 & 11.87 & 14.88 & 14.88 & 23.4 & 23.4 & 23.5 & 23.5 & 23.5 & 23.5 & 23.5 & 23.5 & 23.6 & 23.6 \\
\hline ESF-HD-137-TEMP5-RTD-44 & 0.84 & 11.87 & 15.18 & 15.18 & 23.4 & 23.6 & 23.6 & 23.7 & 23.7 & 23.8 & 23.8 & 23.8 & 23.9 & 23.9 \\
\hline ESF-HD-137-TEMP5-RTD-45 & 0.84 & 11.87 & 15.48 & 15.48 & 23.4 & 23.4 & 23.4 & 23.4 & 23.4 & 23.4 & 23.5 & 23.5 & 23.5 & 23.5 \\
\hline ESF-HD-137-TEMP5-RTD-46 & 0.84 & 11.87 & 15.78 & 15.78 & 23.2 & 23.3 & 23.5 & 23.6 & 23.6 & 23.7 & 23.7 & 23.7 & 23.8 & 23.8 \\
\hline ESF-HD-137-TEMP5-RTD-47 & 0.85 & 11.87 & 16.08 & 16.08 & 23.4 & 23.4 & 23.4 & 23.4 & 23.4 & 23.4 & 23.4 & 23.4 & 23.4 & 23.5 \\
\hline ESF-HD-137-TEMP5-RTD-48 & 0.85 & 11.86 & 16.38 & 16.38 & 23.4 & 23.5 & 23.5 & 23.6 & 23.6 & 23.7 & 23.7 & 23.7 & 23.7 & 23.8 \\
\hline ESF-HD-137-TEMP5-RTD-49 & 0.85 & 11.86 & 16.68 & 16.68 & 23.3 & 23.0 & 23.3 & 23.4 & 23.3 & 23.1 & 23.1 & 23.3 & 23.0 & 23.4 \\
\hline ESF-HD-137-TEMP5-RTD-50 & 0.85 & 11.86 & 16.98 & 16.98 & 23.1 & 23.0 & 23.3 & 23.3 & 23.3 & 23.3 & 23.4 & 23.4 & 23.4 & 23.4 \\
\hline ESF-HD-137-TEMP5-RTD-51 & 0.85 & 11.86 & 17.28 & 17.28 & 23.3 & 23.3 & 23.3 & 23.3 & 23.3 & 23.4 & 23.4 & 23.4 & 23.4 & 23.4 \\
\hline ESF-HD-137-TEMP5-RTD-52 & 0.85 & 11.86 & 17.58 & 17.58 & 23.2 & 23.2 & 23.3 & 23.3 & 23.3 & 23.3 & 23.3 & 23.3 & 23.3 & 23.3 \\
\hline ESF-HD-137-TEMP5-RTD-53 & 0.86 & 11.86 & 17.88 & 17.88 & 27.1 & 27.1 & 27.2 & 27.2 & 27.2 & 27.2 & 27.2 & 27.2 & 27.2 & 27.2 \\
\hline ESF-HD-137-TEMP5-RTD-54 & 0.86 & 11.86 & 18.18 & 18.18 & 23.3 & 23.3 & 23.3 & 23.3 & 23.3 & 23.3 & 23.3 & 23.3 & 23.3 & 23.3 \\
\hline ESF-HD-137-TEMP5-RTD-55 & 0.86 & 11.86 & 18.48 & 18.48 & 23.2 & 23.1 & 23.2 & 23.3 & 23.3 & 23.3 & 23.3 & 23.3 & 23.3 & 23.3 \\
\hline ESF-HD-137-TEMP5-RTD-56 & 0.86 & 11.85 & 18.78 & 18.78 & 23.2 & 22.8 & 23.2 & 23.2 & 23.2 & 23.1 & 23.1 & 23.3 & 23.3 & 23.3 \\
\hline ESF-HD-137-TEMP5-RTD-57 & 0.86 & 11.85 & 19.07 & 19.07 & 23.3 & 23.3 & 23.3 & 23.3 & 23.3 & 23.3 & 23.3 & 23.3 & 23.3 & 23.3 \\
\hline ESF-HD-137-TEMP5-RTD-58 & 0.86 & 11.85 & 19.37 & 19.37 & 22.9 & 22.9 & 23.1 & 23.2 & 23.1 & 23.0 & 23.1 & 23.2 & 23.2 & 23.2 \\
\hline ESF-HD-137-TEMP5-RTD-59 & 0.87 & 11.85 & 19.67 & 19.67 & 23.2 & 23.1 & 23.2 & 23.2 & 23.2 & 23.2 & 23.2 & 23.2 & 23.2 & 23.2 \\
\hline ESF-HD-137-TEMP5-RTD-60 & 0.87 & 11.85 & 19.97 & 19.97 & 23.2 & 23.2 & 23.2 & 23.2 & 23.2 & 23.2 & 23.2 & 23.2 & 23.2 & 23.3 \\
\hline ESF-HD-137-TEMP5-RTD-61 & 0.87 & 11.85 & 20.27 & 20.27 & 23.2 & 23.2 & 23.2 & 23.2 & 23.2 & 23.2 & 23.2 & 23.2 & 23.2 & 23.2 \\
\hline ESF-HD-137-TEMP5-RTD-62 & 0.87 & 11.85 & 20.57 & 20.57 & 23.2 & 23.2 & 23.2 & 23.2 & 23.2 & 23.2 & 23.2 & 23.2 & 23.2 & 23.2 \\
\hline ESF-HD-137-TEMP5-RTD-63 & 0.87 & 11.85 & 20.87 & 20.87 & 23.2 & 23.2 & 23.2 & 23.2 & 23.2 & 23.2 & 23.2 & 23.2 & 23.2 & 23.2 \\
\hline ESF-HD-137-TEMP5-RTD-64 & 0.87 & 11.85 & 21.17 & 21.17 & 23.1 & 23.1 & 23.1 & 23.1 & 23.1 & 23.1 & 23.1 & 23.2 & 23.2 & 23.2 \\
\hline ESF-HD-137-TEMP5-RTD-65 & 0.87 & 11.84 & 21.47 & 21.47 & 23.2 & 23.2 & 23.2 & 23.2 & 23.2 & 23.2 & 23.2 & 23.2 & 23.2 & 23.2 \\
\hline ESF-HD-137-TEMP5-RTD-66 & 0.88 & 11.84 & 21.77 & 21.77 & 20.5 & 20.6 & 20.7 & 20.7 & 20.8 & 20.8 & 20.8 & 20.8 & 20.9 & 20.9 \\
\hline ESF-HD-137-TEMP5-RTD-67 & 0.88 & 11.84 & 22.07 & 22.07 & 22.9 & 23.0 & 23.1 & 23.2 & 23.2 & 23.2 & 23.2 & 23.2 & 23.1 & 23.1 \\
\hline \multicolumn{15}{|l|}{ BH 138 Gage ID } \\
\hline ESF-HD-138-TEMP6-RTD-1 & -1.72 & 11.89 & 1.70 & 2.42 & 30.2 & 62.0 & 71.9 & 78.8 & 85.1 & 90.1 & 93.9 & 98.0 & 102.3 & 104.0 \\
\hline ESF-HD-138-TEMP6-RTD-2 & -1.93 & 11.88 & 1.91 & 2.72 & 30.2 & 60.1 & 69.9 & 76.8 & 83.3 & 88.2 & 92.7 & 96.4 & 100.7 & 102.7 \\
\hline ESF-HD-138-TEMP6-RTD-3 & -2.15 & 11.88 & 2.12 & 3.02 & 29.9 & 48.0 & 58.1 & 65.3 & 71.8 & 76.9 & 81.4 & 85.6 & 89.5 & 91.9 \\
\hline
\end{tabular}




\begin{tabular}{|c|c|c|c|c|c|c|c|c|c|c|c|c|c|c|}
\hline \multirow{5}{*}{ ESF-HD-138-TEMP6-RTD-4 } & \multicolumn{14}{|c|}{ Temperature Data Obtained from the DST } \\
\hline & \multicolumn{14}{|c|}{ Days after startup } \\
\hline & \multicolumn{4}{|c|}{ As Built Locations (m) } & 0 & 7 & 17 & 27 & 38 & 48 & 58 & 69 & 79 & 87 \\
\hline & $\mathbf{x}$ & $\mathbf{y}$ & $\mathbf{z}$ & D & \multicolumn{10}{|c|}{$\left({ }^{\circ} \mathrm{C}\right)$} \\
\hline & -2.36 & & 2.33 & & 29.7 & 40.7 & & & & 68.8 & 73.4 & 7.8 & 81.6 & 4.3 \\
\hline ESF-HD-138-TEMP6-RTD-5 & -2.57 & 86 & & 3.6 & 29.6 & .1 & & & & & .9 & .4 & 75.2 & \\
\hline ESF-HD-138-TEMP6-RT & .78 & 86 & & 3.92 & 29.4 & 0 & & & & 57.1 & 61.4 & 5.9 & 69.7 & \\
\hline ESF-HD-138-TEMP6-RT & .99 & .85 & 97 & 4.21 & 29.1 & .0 & 6.8 & .3 & .8 & 52.4 & 56.6 & 1.0 & 64.7 & 7.5 \\
\hline ESF-HD-138-TEMP6-RTD-8 & .21 & 85 & & 4.52 & 28.8 & .7 & 2 & .0 & .1 & 3.5 & .5 & 6.8 & 60.4 & 3.2 \\
\hline ESF-HD- & 3.42 & & & 4.82 & 28.5 & 28.8 & & & .8 & & 7 & 2.8 & 56.3 & \\
\hline ESF-HD- & 3.63 & & & 5.12 & 28.1 & & & & 3.1 & & & .4 & 52.8 & \\
\hline ESF-HD-138-TEMP6-RTD-11 & 3.84 & & & 5.42 & 27.8 & & & & 35.7 & 39.1 & .5 & 46.1 & 49.4 & \\
\hline ESF-HD-138-TEMP6-RTD-12 & 4.06 & & & 5.72 & 27.4 & & & & 3.8 & & & 43.4 & 46.5 & \\
\hline SF-HD-1 & & & & 6.02 & 27 & & & & & & & 0.9 & 43.8 & \\
\hline-14 & & & & 6.32 & 26.8 & & & & & & & 3.9 & 41.6 & \\
\hline-15 & & & & 6.62 & 26.5 & & & & & & & 36.9 & 39.5 & \\
\hline-16 & .91 & & & 6.92 & 26.2 & & & & 8.8 & & & 5.3 & 37.7 & \\
\hline$D-17$ & 12 & & 5.09 & 7.22 & 26.0 & 26.0 & & 0.1 & 8.0 & 29.6 & & 3.8 & 36.0 & \\
\hline ESF-H & 33 & & 5.30 & 7.52 & 25.7 & & & 20.0 & 27.3 & 28.6 & & 2.4 & 34.5 & \\
\hline ESF-H & .54 & & & 7.82 & 25.5 & & & & & & & 1.3 & 33.2 & \\
\hline ESF-H & .76 & & & .12 & 23.7 & & & & & & & 8.2 & 9.9 & \\
\hline & & & & & & & & & & & & & & \\
\hline & & & & & & & & & & & & & & \\
\hline & & & & & & & & & & & & & 9.1 & \\
\hline-24 & & & & & & & & & & & & & 8.3 & \\
\hline-25 & & & & 9.62 & & & & & & & & 0.1 & & \\
\hline ESF-HD-138-TEMP6-RTD-26 & & & & & & & & & & & & & & \\
\hline ESF-HD-138-TEMP6-RTD-27 & & & & 10.22 & & & & & & & & & & \\
\hline & & & & & & & & & & & & & & \\
\hline & & & & & & & & & & & & & & \\
\hline & & & & & & & & & & & & & & \\
\hline & & & & 11.42 & & & & & & & & & & \\
\hline & & & & 11.72 & 23.9 & & & & & & & & & \\
\hline & & 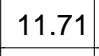 & & 12.03 & 23.8 & & & & & & & & & \\
\hline ESF-HD-138-TEMP6-RTD-34 & 3.73 & & & 12.33 & 23.6 & & & & & & & 24.3 & 24.5 & \\
\hline ESF-HD-138-TEMP6-RTD-35 & 3.94 & & & 12.62 & 23.5 & & & & 23.8 & & & 24.1 & 24.4 & \\
\hline ESF-HD-138-TEMP6-RTD-36 & .15 & & & 12.92 & 23.6 & & & & & & & 24.0 & 24.2 & \\
\hline & -9.36 & & 9.34 & 13.22 & 23.7 & & & & 24.0 & & & & 24.5 & \\
\hline & -9.58 & 11.00 & & & 23.4 & & & & & & & 23.9 & 24.0 & \\
\hline & -9.79 & 11.00 & & 13.82 & 23.5 & & & & 23.0 & & 22.9 & 23.8 & 23.9 & \\
\hline SF-HD-138-TEMP6-RTD-40 & -10.00 & 11.67 & & 14.12 & 23.4 & 23.0 & 23.5 & 23.5 & 23.2 & 22.8 & 23.0 & 23.5 & 23.6 & \\
\hline
\end{tabular}


First Quarter Report of Temperature Measurements During the Heating Phase of the Drift-Scale Test

\begin{tabular}{|c|c|c|c|c|c|c|c|c|c|c|c|c|c|c|}
\hline \multirow{5}{*}{\begin{tabular}{|l} 
ESF-HD-138-TEMP6-RTD-41 \\
\end{tabular}} & \multicolumn{14}{|c|}{ Temperature Data Obtained from the DST } \\
\hline & \multicolumn{14}{|c|}{ Days after startup } \\
\hline & \multicolumn{4}{|c|}{ As Built Locations (m) } & 0 & 7 & 17 & 27 & 38 & 48 & 58 & 69 & 79 & 87 \\
\hline & \multirow{2}{*}{\begin{tabular}{|c|}
$\mathbf{X}$ \\
-10.21 \\
\end{tabular}} & \multirow{2}{*}{\begin{tabular}{|c|}
$\mathbf{y}$ \\
11.67 \\
\end{tabular}} & \multirow{2}{*}{\begin{tabular}{|c|}
$\mathbf{z}$ \\
10.18 \\
\end{tabular}} & \multirow{2}{*}{$\begin{array}{c}\text { D } \\
14.42\end{array}$} & \multicolumn{10}{|c|}{$\left({ }^{\circ} \mathrm{C}\right)$} \\
\hline & & & & & 23.5 & 23.3 & 23.3 & 23.3 & 23.3 & 23.3 & 23.3 & 23.4 & 23.4 & 23.5 \\
\hline ESF-HD-138-TEMP6-RTD-42 & -10.43 & 11.66 & 10.40 & 14.73 & 23.5 & 23.3 & 23.3 & 23.3 & 23.3 & 23.3 & 23.3 & 23.3 & 23.4 & 23.4 \\
\hline ESF-HD-138-TEMP6-RTD-43 & -10.64 & 11.65 & 10.61 & 15.03 & 23.4 & 23.3 & 23.3 & 23.3 & 23.2 & 23.2 & 23.3 & 23.3 & 23.3 & 23.4 \\
\hline ESF-HD-138-TEMP6-RTD-44 & -10.85 & 11.65 & 10.82 & 15.32 & 23.4 & 23.4 & 23.5 & 23.5 & 23.5 & 23.5 & 23.5 & 23.6 & 23.6 & 23.7 \\
\hline ESF-HD-138-TEMP6-RTD-45 & -11.06 & 11.64 & 11.03 & 15.62 & 23.4 & 23.3 & 23.2 & 23.2 & 23.2 & 23.2 & 23.2 & 23.2 & 23.2 & 23.3 \\
\hline ESF-HD-138-TEMP6-RTD-46 & -11.28 & 11.64 & 11.25 & 15.93 & 23.2 & 23.3 & 23.4 & 23.4 & 23.5 & 23.5 & 23.5 & 23.5 & 23.5 & 23.6 \\
\hline ESF-HD-138-TEMP6-RTD-47 & -11.49 & 11.63 & 11.46 & 16.23 & 23.6 & 23.5 & 23.6 & 23.6 & 23.6 & 23.7 & 23.7 & 23.9 & 24.0 & 23.6 \\
\hline ESF-HD-138-TEMP6-RTD-48 & -11.70 & 11.63 & 11.67 & 16.53 & 23.3 & 23.4 & 23.4 & 23.4 & 23.4 & 23.4 & 23.4 & 23.5 & 23.5 & 23.5 \\
\hline ESF-HD-138-TEMP6-RTD-49 & -11.91 & 11.62 & 11.88 & 16.82 & 23.3 & 23.3 & 23.4 & 23.4 & 23.4 & 23.4 & 23.5 & 23.5 & 23.5 & 23.5 \\
\hline ESF-HD-138-TEMP6-RTD-50 & -12.13 & 11.62 & 12.09 & 17.13 & 23.3 & 23.3 & 23.4 & 23.4 & 23.4 & 23.4 & 23.4 & 23.4 & 23.5 & 23.5 \\
\hline ESF-HD-138-TEMP6-RTD-51 & -12.34 & 11.61 & 12.31 & 17.43 & 23.4 & 23.4 & 23.4 & 23.4 & 23.4 & 23.4 & 23.4 & 23.4 & 23.4 & 23.4 \\
\hline ESF-HD-138-TEMP6-RTD-52 & -12.55 & 11.60 & 12.52 & 17.73 & 23.3 & 23.2 & 23.3 & 23.4 & 23.4 & 23.4 & 23.4 & 23.4 & 23.4 & 23.4 \\
\hline ESF-HD-138-TEMP6-RTD-53 & -12.76 & 11.60 & 12.73 & 18.02 & 23.3 & 23.1 & 23.1 & 23.1 & 23.0 & 23.0 & 23.0 & 23.0 & 23.0 & 23.0 \\
\hline ESF-HD-138-TEMP6-RTD-54 & -12.97 & 11.59 & 12.94 & 18.32 & 23.1 & 23.3 & 23.3 & 23.4 & 23.4 & 23.4 & 23.4 & 23.4 & 23.4 & 23.4 \\
\hline ESF-HD-138-TEMP6-RTD-55 & -13.19 & 11.59 & 13.15 & 18.63 & 23.3 & 23.4 & 23.4 & 23.4 & 23.4 & 23.4 & 23.4 & 23.4 & 23.4 & 23.4 \\
\hline ESF-HD-138-TEMP6-RTD-56 & -13.40 & 11.58 & 13.37 & 18.93 & 23.3 & 23.4 & 23.4 & 23.4 & 23.4 & 23.4 & 23.4 & 23.4 & 23.4 & 23.4 \\
\hline ESF-HD-138-TEMP6-RTD-57 & -13.61 & 11.58 & 13.58 & 19.23 & 23.1 & 23.4 & 23.5 & 23.6 & 23.6 & 23.6 & 23.7 & 23.7 & 23.7 & 23.7 \\
\hline ESF-HD-138-TEMP6-RTD-58 & -13.82 & 11.57 & 13.79 & 19.52 & 23.3 & 23.3 & 23.3 & 23.3 & 23.3 & 23.3 & 23.3 & 23.3 & 23.3 & 23.3 \\
\hline ESF-HD-138-TEMP6-RTD-59 & -14.04 & 11.57 & 14.00 & 19.83 & 23.3 & 23.3 & 23.3 & 23.3 & 23.3 & 23.3 & 23.3 & 23.3 & 23.3 & 23.3 \\
\hline ESF-HD-138-TEMP6-RTD-60 & -14.25 & 11.56 & 14.22 & 20.13 & 23.3 & 23.3 & 23.3 & 23.3 & 23.3 & 23.3 & 23.3 & 23.3 & 23.3 & 23.3 \\
\hline ESF-HD-138-TEMP6-RTD-61 & -14.46 & 11.56 & 14.43 & 20.43 & 23.3 & 23.3 & 23.3 & 23.3 & 23.3 & 23.3 & 23.3 & 23.3 & 23.3 & 23.3 \\
\hline ESF-HD-138-TEMP6-RTD-62 & -14.67 & 11.55 & 14.64 & 20.73 & 23.3 & 23.3 & 23.3 & 23.3 & 23.3 & 23.3 & 23.3 & 23.3 & 23.3 & 23.3 \\
\hline ESF-HD-138-TEMP6-RTD-63 & -14.89 & 11.54 & 14.85 & 21.03 & 23.3 & 23.3 & 23.3 & 23.3 & 23.3 & 23.4 & 23.4 & 23.4 & 23.4 & 23.4 \\
\hline ESF-HD-138-TEMP6-RTD-64 & -15.10 & 11.54 & 15.06 & 21.33 & 23.2 & 23.2 & 23.2 & 23.2 & 23.2 & 23.2 & 23.2 & 23.2 & 23.2 & 23.2 \\
\hline ESF-HD-138-TEMP6-RTD-65 & -15.31 & 11.53 & 15.28 & 21.63 & 23.3 & 23.3 & 23.3 & 23.3 & 23.3 & 23.3 & 23.3 & 23.3 & 23.3 & 23.3 \\
\hline ESF-HD-138-TEMP6-RTD-66 & -15.52 & 11.53 & 15.49 & 21.93 & 23.0 & 23.1 & 23.2 & 23.2 & 23.2 & 23.2 & 23.2 & 23.2 & 23.2 & 23.2 \\
\hline ESF-HD-138-TEMP6-RTD-67 & -15.73 & 11.52 & 15.70 & 22.22 & 23.2 & 23.2 & 23.3 & 23.3 & 23.3 & 23.3 & 23.3 & 23.3 & 23.3 & 23.3 \\
\hline \multicolumn{15}{|l|}{ BH 139 Gage ID } \\
\hline ESF-HD-139-TEMP7-RTD-1 & -2.31 & 11.89 & -0.02 & 2.31 & 30.1 & 60.6 & 70.6 & 77.6 & 84.1 & 89.0 & 93.5 & 97.3 & 101.4 & 103.5 \\
\hline ESF-HD-139-TEMP7-RTD-2 & -2.61 & 11.89 & -0.02 & 2.61 & 23.9 & 46.3 & 61.2 & 70.7 & 75.1 & 82.9 & 87.8 & 92.5 & 96.1 & 97.7 \\
\hline ESF-HD-139-TEMP7-RTD-3 & -2.91 & 11.89 & -0.01 & 2.91 & 29.8 & 46.4 & 58.7 & 65.8 & 73.4 & 79.0 & 83.9 & 88.5 & 92.4 & 94.8 \\
\hline ESF-HD-139-TEMP7-RTD-4 & -3.21 & 11.89 & -0.01 & 3.21 & 29.7 & 41.3 & 54.2 & 61.3 & 69.5 & 75.3 & 80.5 & 85.3 & 89.5 & 92.2 \\
\hline ESF-HD-139-TEMP7-RTD-5 & -3.51 & 11.89 & -0.01 & 3.51 & 29.5 & 39.1 & 52.1 & 59.3 & 67.9 & 73.8 & 79.1 & 84.0 & 88.5 & 91.4 \\
\hline ESF-HD-139-TEMP7-RTD-6 & -3.81 & 11.89 & -0.01 & 3.81 & 29.2 & 37.8 & 50.8 & 58.1 & 67.1 & 73.2 & 78.7 & 83.9 & 88.6 & 91.7 \\
\hline ESF-HD-139-TEMP7-RTD-7 & -4.11 & 11.90 & 0.00 & 4.11 & 28.9 & 37.4 & 50.4 & 58.1 & 67.3 & 73.6 & 79.3 & 84.6 & 89.4 & 92.8 \\
\hline ESF-HD-139-TEMP7-RTD-8 & -4.41 & 11.90 & 0.00 & 4.41 & 28.6 & 38.1 & 50.9 & 59.5 & 68.8 & 75.3 & 81.2 & 86.7 & 91.5 & 95.2 \\
\hline ESF-HD-139-TEMP7-RTD-9 & -4.71 & 11.90 & 0.00 & 4.71 & 28.3 & 38.7 & 51.6 & 60.9 & 70.1 & 76.9 & 82.8 & 88.4 & 93.2 & 96.2 \\
\hline
\end{tabular}




\begin{tabular}{|c|c|c|c|c|c|c|c|c|c|c|c|c|c|c|}
\hline & \multicolumn{14}{|c|}{ Temperature Data Obtained from the DST } \\
\hline & \multicolumn{14}{|c|}{ Days after startup } \\
\hline & \multicolumn{4}{|c|}{ As Built Locations (m) } & 0 & 7 & 17 & 27 & 38 & 48 & 58 & 69 & 79 & 87 \\
\hline & $\mathbf{x}$ & $\mathbf{y}$ & $\mathbf{z}$ & D & \multicolumn{10}{|c|}{$\left({ }^{\circ} \mathbf{C}\right)$} \\
\hline ESF-HD-139-TEMP7-RTD-10 & -5.01 & 11.90 & 0.00 & 5.01 & 27.9 & 39.7 & 52.9 & 63.0 & 72.3 & 79.4 & 85.3 & 90.8 & 95.5 & 96.8 \\
\hline ESF-HD-139-TEMP7-RTD-11 & -5.31 & 11.90 & 0.01 & 5.31 & 27.7 & 40.2 & 53.6 & 64.2 & 73.5 & 80.7 & 86.7 & 91.9 & 95.7 & 97.0 \\
\hline ESF-HD-139-TEMP7-RTD-12 & -5.61 & 11.90 & 0.01 & 5.61 & 27.3 & 40.7 & 54.7 & 65.7 & 75.1 & 82.5 & 88.4 & 93.2 & 96.1 & 97.1 \\
\hline ESF-HD-139-TEMP7-RTD-13 & -5.91 & 11.90 & 0.01 & 5.91 & 27.0 & 40.6 & 55.0 & 66.1 & 75.6 & 83.1 & 88.8 & 93.6 & 96.2 & 97.1 \\
\hline ESF-HD-139-TEMP7-RTD-14 & -6.21 & 11.90 & 0.01 & 6.21 & 26.3 & 40.6 & 55.5 & 66.8 & 76.4 & 83.8 & 89.4 & 94.0 & 96.4 & 97.2 \\
\hline ESF-HD-139-TEMP7-RTD-15 & -6.50 & 11.90 & 0.02 & 6.50 & 26.5 & 40.4 & 55.6 & 66.9 & 76.5 & 83.8 & 89.4 & 94.1 & 96.6 & 97.2 \\
\hline ESF-HD-139-TEMP7-RTD-16 & -6.80 & 11.90 & 0.02 & 6.80 & 26.2 & 40.5 & 55.9 & 67.2 & 76.9 & 84.1 & 89.9 & 95.0 & 96.7 & 97.1 \\
\hline ESF-HD-139-TEMP7-RTD-17 & -7.10 & 11.90 & 0.02 & 7.10 & 26.0 & 40.0 & 55.5 & 66.7 & 76.4 & 83.6 & 89.7 & 95.5 & 96.7 & 97.1 \\
\hline ESF-HD-139-TEMP7-RTD-18 & -7.40 & 11.91 & 0.02 & 7.40 & 25.7 & 39.8 & 55.3 & 66.6 & 76.4 & 83.7 & 90.2 & 95.8 & 96.6 & 97.2 \\
\hline ESF-HD-139-TEMP7-RTD-19 & -7.70 & 11.91 & 0.03 & 7.70 & 25.5 & 39.3 & 54.6 & 65.9 & 75.7 & 82.8 & 89.3 & 94.5 & 96.4 & 96.9 \\
\hline ESF-HD-139-TEMP7-RTD-20 & -8.00 & 11.91 & 0.03 & 8.00 & 25.3 & 38.8 & 53.8 & 65.1 & 74.7 & 81.7 & 87.7 & 93.2 & 96.0 & 96.8 \\
\hline ESF-HD-139-TEMP7-RTD-21 & -8.30 & 11.91 & 0.03 & 8.30 & 25.2 & 37.9 & 52.6 & 64.0 & 73.5 & 80.3 & 86.0 & 91.7 & 95.5 & 96.7 \\
\hline ESF-HD-139-TEMP7-RTD-22 & -8.60 & 11.91 & 0.03 & 8.60 & 25.0 & 37.1 & 51.6 & 63.1 & 72.4 & 79.1 & 84.4 & 90.1 & 94.7 & 96.3 \\
\hline ESF-HD-139-TEMP7-RTD-23 & -8.90 & 11.91 & 0.04 & 8.90 & 24.8 & 36.6 & 51.0 & 62.7 & 71.9 & 78.6 & 83.6 & 89.1 & 93.6 & 95.6 \\
\hline ESF-HD-139-TEMP7-RTD-24 & -9.20 & 11.91 & 0.04 & 9.20 & 24.7 & 36.9 & 51.4 & 63.4 & 72.6 & 79.3 & 83.8 & 89.1 & 93.5 & 94.1 \\
\hline ESF-HD-139-TEMP7-RTD-25 & -9.50 & 11.91 & 0.04 & 9.50 & 24.6 & 37.0 & 51.8 & 64.0 & 73.5 & 80.2 & 84.4 & 89.6 & 93.9 & 94.2 \\
\hline ESF-HD-139-TEMP7-RTD-26 & -9.80 & 11.91 & 0.04 & 9.80 & 24.1 & 37.8 & 53.5 & 66.1 & 75.7 & 82.5 & 86.1 & 91.4 & 95.1 & 94.7 \\
\hline ESF-HD-139-TEMP7-RTD-27 & -10.10 & 11.91 & 0.05 & 10.10 & 24.3 & 38.2 & 54.6 & 67.4 & 77.1 & 84.0 & 87.4 & 92.7 & 95.4 & 95.5 \\
\hline ESF-HD-139-TEMP7-RTD-28 & -10.40 & 11.92 & 0.05 & 10.40 & 24.3 & 39.3 & 56.4 & 69.1 & 79.0 & 85.7 & 89.8 & 94.5 & 96.4 & 96.5 \\
\hline ESF-HD-139-TEMP7-RTD-29 & -10.70 & 11.92 & 0.05 & 10.70 & 24.2 & 39.5 & 57.0 & 69.7 & 79.8 & 86.2 & 90.8 & 94.9 & 96.5 & 96.7 \\
\hline ESF-HD-139-TEMP7-RTD-30 & -11.00 & 11.92 & 0.05 & 11.00 & 24.1 & 39.9 & 57.9 & 70.7 & 80.7 & 86.9 & 91.6 & 95.6 & 97.0 & 96.6 \\
\hline ESF-HD-139-TEMP7-RTD-31 & -11.30 & 11.92 & 0.06 & 11.30 & 23.4 & 39.1 & 57.3 & 69.7 & 80.0 & 86.0 & 91.0 & 95.3 & 97.3 & 96.2 \\
\hline ESF-HD-139-TEMP7-RTD-32 & -11.60 & 11.92 & 0.06 & 11.60 & 24.1 & 40.3 & 58.1 & 70.5 & 79.9 & 86.0 & 90.7 & 94.7 & 97.5 & 96.1 \\
\hline ESF-HD-139-TEMP7-RTD-33 & -11.90 & 11.92 & 0.06 & 11.90 & 23.9 & 40.0 & 57.5 & 69.6 & 78.7 & 84.8 & 89.5 & 93.6 & 96.8 & 95.4 \\
\hline ESF-HD-139-TEMP7-RTD-34 & -12.20 & 11.92 & 0.06 & 12.20 & 24.0 & 39.7 & 56.5 & 68.2 & 76.9 & 83.1 & 88.0 & 92.5 & 96.5 & 93.8 \\
\hline ESF-HD-139-TEMP7-RTD-35 & -12.50 & 11.92 & 0.07 & 12.50 & 23.9 & 39.2 & 55.3 & 66.5 & 74.8 & 80.9 & 85.8 & 90.7 & 95.3 & 92.2 \\
\hline ESF-HD-139-TEMP7-RTD-36 & -12.80 & 11.92 & 0.07 & 12.80 & 23.9 & 38.3 & 53.2 & 63.6 & 71.4 & 77.2 & 81.9 & 87.3 & 92.7 & 88.1 \\
\hline ESF-HD-139-TEMP7-RTD-37 & -13.10 & 11.92 & 0.07 & 13.10 & 21.2 & 36.4 & 50.7 & 60.6 & 67.8 & 72.7 & 77.4 & 83.1 & 88.9 & 84.5 \\
\hline ESF-HD-139-TEMP7-RTD-38 & -13.40 & 11.92 & 0.07 & 13.40 & 23.3 & 33.5 & 46.8 & 56.0 & 62.2 & 67.2 & 71.3 & 76.6 & 81.7 & 78.4 \\
\hline ESF-HD-139-TEMP7-RTD-39 & -13.70 & 11.93 & 0.08 & 13.70 & 23.8 & 32.8 & 43.2 & 51.5 & 57.5 & 62.3 & 66.3 & 70.8 & 76.4 & 73.7 \\
\hline ESF-HD-139-TEMP7-RTD-40 & -14.00 & 11.93 & 0.08 & 14.00 & 23.7 & 30.0 & 38.7 & 46.1 & 51.6 & 56.0 & 59.7 & 64.0 & 69.2 & 67.5 \\
\hline ESF-HD-139-TEMP7-RTD-41 & -14.30 & 11.93 & 0.08 & 14.30 & 23.7 & 28.1 & 35.4 & 42.0 & 47.0 & 51.1 & 54.7 & 58.7 & 63.4 & 62.8 \\
\hline ESF-HD-139-TEMP7-RTD-42 & -14.60 & 11.93 & 0.08 & 14.60 & 23.7 & 26.2 & 31.7 & 37.0 & 41.6 & 45.2 & 48.5 & 52.1 & 56.1 & 56.6 \\
\hline ESF-HD-139-TEMP7-RTD-43 & -14.90 & 11.93 & 0.09 & 14.90 & 23.8 & 25.3 & 29.7 & 34.2 & 38.4 & 41.7 & 44.8 & 48.0 & 51.5 & 52.7 \\
\hline ESF-HD-139-TEMP7-RTD-44 & -15.20 & 11.93 & 0.09 & 15.20 & 23.7 & 24.5 & 27.5 & 31.0 & 34.6 & 37.5 & 40.3 & 43.2 & 46.1 & 47.7 \\
\hline ESF-HD-139-TEMP7-RTD-45 & -15.50 & 11.93 & 0.09 & 15.50 & 23.7 & 24.1 & 26.4 & 29.3 & 32.5 & 35.1 & 37.7 & 40.3 & 42.9 & 44.7 \\
\hline ESF-HD-139-TEMP7-RTD-46 & -15.79 & 11.93 & 0.09 & 15.79 & 23.7 & 23.9 & 25.4 & 27.5 & 30.1 & 32.4 & 34.6 & 36.9 & 39.2 & 40.9 \\
\hline
\end{tabular}


First Quarter Report of Temperature Measurements During the Heating Phase of the Drift-Scale Test

\begin{tabular}{|c|c|c|c|c|c|c|c|c|c|c|c|c|c|c|}
\hline \multirow{5}{*}{\begin{tabular}{|l} 
\\
ESF-HD-139-TEMP7-RTD-47 \\
\end{tabular}} & \multicolumn{14}{|c|}{ Temperature Data Obtained from the DST } \\
\hline & \multicolumn{14}{|c|}{ Days after startup } \\
\hline & \multicolumn{4}{|c|}{ As Built Locations (m) } & 0 & 7 & 17 & 27 & 38 & 48 & 58 & 69 & 79 & 87 \\
\hline & \multirow{2}{*}{$\begin{array}{c}\mathbf{x} \\
-16.09 \\
\end{array}$} & \multirow{2}{*}{\begin{tabular}{|c|}
$\mathbf{y}$ \\
11.93 \\
\end{tabular}} & \multirow{2}{*}{$\begin{array}{c}z \\
0.10 \\
\end{array}$} & \multirow{2}{*}{$\begin{array}{c}\mathbf{D} \\
16.09\end{array}$} & \multicolumn{10}{|c|}{$\left({ }^{\circ} \mathbf{C}\right)$} \\
\hline & & & & & 23.7 & 23.8 & 24.9 & 26.6 & 28.9 & 30.9 & 32.9 & 35.1 & 37.1 & 38.7 \\
\hline ESF-HD-139-TEMP7-RTD-48 & -16.39 & 11.93 & 0.10 & 16.39 & 23.4 & 23.6 & 24.3 & 25.5 & 27.3 & 29.0 & 30.7 & 32.5 & 34.3 & 35.7 \\
\hline ESF-HD-139-TEMP7-RTD-49 & -16.69 & 11.93 & 0.10 & 16.69 & 23.5 & 23.6 & 24.1 & 25.1 & 26.6 & 28.1 & 29.6 & 31.3 & 32.9 & 34.2 \\
\hline ESF-HD-139-TEMP7-RTD-50 & -16.99 & 11.94 & 0.10 & 16.99 & 23.7 & 23.6 & 23.9 & 24.6 & 25.7 & 26.9 & 28.2 & 29.6 & 31.0 & 32.1 \\
\hline ESF-HD-139-TEMP7-RTD-51 & -17.29 & 11.94 & 0.11 & 17.29 & 23.7 & 23.7 & 23.8 & 24.4 & 25.3 & 26.3 & 27.4 & 28.8 & 30.0 & 31.0 \\
\hline ESF-HD-139-TEMP7-RTD-52 & -17.59 & 11.94 & 0.11 & 17.59 & 23.6 & 23.6 & 23.7 & 24.1 & 24.8 & 25.6 & 26.5 & 27.6 & 28.6 & 29.5 \\
\hline ESF-HD-139-TEMP7-RTD-53 & -17.89 & 11.94 & 0.11 & 17.89 & 23.7 & 23.7 & 23.8 & 24.0 & 24.5 & 25.2 & 26.0 & 27.0 & 28.0 & 28.8 \\
\hline ESF-HD-139-TEMP7-RTD-54 & -18.19 & 11.94 & 0.11 & 18.19 & 22.9 & 23.5 & 23.7 & 23.9 & 24.2 & 24.7 & 25.4 & 26.2 & 26.9 & 27.7 \\
\hline ESF-HD-139-TEMP7-RTD-55 & -18.49 & 11.94 & 0.12 & 18.49 & 23.7 & 23.7 & 23.7 & 23.8 & 24.2 & 24.6 & 25.1 & 25.8 & 26.6 & 27.2 \\
\hline ESF-HD-139-TEMP7-RTD-56 & -18.79 & 11.94 & 0.12 & 18.79 & 22.8 & 23.5 & 23.6 & 23.7 & 24.0 & 24.3 & 24.7 & 25.3 & 25.9 & 26.4 \\
\hline ESF-HD-139-TEMP7-RTD-57 & -19.09 & 11.94 & 0.12 & 19.09 & 23.6 & 23.6 & 23.6 & 23.7 & 23.9 & 24.2 & 24.5 & 25.0 & 25.5 & 26.0 \\
\hline ESF-HD-139-TEMP7-RTD-58 & -19.39 & 11.94 & 0.12 & 19.39 & 22.5 & 22.7 & 23.6 & 23.7 & 23.8 & 24.0 & 24.3 & 24.7 & 25.1 & 25.5 \\
\hline ESF-HD-139-TEMP7-RTD-59 & -19.69 & 11.94 & 0.13 & 19.69 & 23.5 & 23.6 & 23.7 & 23.7 & 23.8 & 24.0 & 24.2 & 24.6 & 24.9 & 25.3 \\
\hline ESF-HD-139-TEMP7-RTD-60 & -19.99 & 11.94 & 0.13 & 19.99 & 23.5 & 23.5 & 23.6 & 23.7 & 23.7 & 23.8 & 24.1 & 24.4 & 24.7 & 25.0 \\
\hline ESF-HD-139-TEMP7-RTD-61 & -20.29 & 11.95 & 0.13 & 20.29 & 23.7 & 23.8 & 23.8 & 23.8 & 23.9 & 24.0 & 24.1 & 24.4 & 24.6 & 24.9 \\
\hline ESF-HD-139-TEMP7-RTD-62 & -20.59 & 11.95 & 0.13 & 20.59 & 23.7 & 23.7 & 23.7 & 23.8 & 23.8 & 23.9 & 24.0 & 24.2 & 24.4 & 24.6 \\
\hline ESF-HD-139-TEMP7-RTD-63 & -20.89 & 11.95 & 0.13 & 20.89 & 23.8 & 23.8 & 23.8 & 23.9 & 23.9 & 24.0 & 24.1 & 24.2 & 24.4 & 24.5 \\
\hline ESF-HD-139-TEMP7-RTD-64 & -21.19 & 11.95 & 0.14 & 21.19 & 23.8 & 23.8 & 23.9 & 23.9 & 23.9 & 24.0 & 24.1 & 24.2 & 24.3 & 24.4 \\
\hline ESF-HD-139-TEMP7-RTD-65 & -21.49 & 11.95 & 0.14 & 21.49 & 23.4 & 23.8 & 23.9 & 24.0 & 24.0 & 24.1 & 24.1 & 24.2 & 24.3 & 24.4 \\
\hline ESF-HD-139-TEMP7-RTD-66 & -21.79 & 11.95 & 0.14 & 21.79 & 23.5 & 23.8 & 23.9 & 24.0 & 24.0 & 24.0 & 24.1 & 24.2 & 24.2 & 24.3 \\
\hline ESF-HD-139-TEMP7-RTD-67 & -22.09 & 11.95 & 0.14 & 22.09 & 23.9 & 23.9 & 24.0 & 24.0 & 24.1 & 24.1 & 24.1 & 24.2 & 24.2 & 24.3 \\
\hline \multicolumn{15}{|l|}{ BH 140 Gage ID } \\
\hline ESF-HD-140-TEMP8-RTD-1 & -2.26 & 11.90 & -1.90 & 2.95 & 29.8 & 38.4 & 47.4 & 54.3 & 60.5 & 65.6 & 70.2 & 74.6 & 78.3 & 80.9 \\
\hline ESF-HD-140-TEMP8-RTD-2 & -2.49 & 11.90 & -2.09 & 3.25 & 29.6 & 35.0 & 43.0 & 49.6 & 55.7 & 60.8 & 65.3 & 69.9 & 73.6 & 76.4 \\
\hline ESF-HD-140-TEMP8-RTD-3 & -2.72 & 11.90 & -2.28 & 3.55 & 29.3 & 32.3 & 39.1 & 45.3 & 51.2 & 56.2 & 60.7 & 65.3 & 69.1 & 71.9 \\
\hline ESF-HD-140-TEMP8-RTD-4 & -2.95 & 11.90 & -2.48 & 3.85 & 28.8 & 30.6 & 36.3 & 42.0 & 47.6 & 52.4 & 56.9 & 61.4 & 65.1 & 68.0 \\
\hline ESF-HD-140-TEMP8-RTD-5 & -3.18 & 11.90 & -2.67 & 4.15 & 28.7 & 29.5 & 34.0 & 39.1 & 44.5 & 49.1 & 53.5 & 57.9 & 61.7 & 64.6 \\
\hline ESF-HD-140-TEMP8-RTD-6 & -3.41 & 11.90 & -2.87 & 4.46 & 28.4 & 28.7 & 32.2 & 36.7 & 41.7 & 46.2 & 50.4 & 54.8 & 58.5 & 61.4 \\
\hline ESF-HD-140-TEMP8-RTD-7 & -3.64 & 11.89 & -3.06 & 4.76 & 28.0 & 28.1 & 30.7 & 34.7 & 39.4 & 43.6 & 47.7 & 51.9 & 55.6 & 58.4 \\
\hline ESF-HD-140-TEMP8-RTD-8 & -3.87 & 11.89 & -3.26 & 5.06 & 27.7 & 27.7 & 29.6 & 33.1 & 37.3 & 41.3 & 45.2 & 49.3 & 52.9 & 55.7 \\
\hline ESF-HD-140-TEMP8-RTD-9 & -4.10 & 11.89 & -3.45 & 5.36 & 27.4 & 27.4 & 28.8 & 31.7 & 35.5 & 39.3 & 43.0 & 47.0 & 50.4 & 53.1 \\
\hline ESF-HD-140-TEMP8-RTD-10 & -4.33 & 11.89 & -3.65 & 5.66 & 27.0 & 27.0 & 28.1 & 30.5 & 34.0 & 37.5 & 41.0 & 44.8 & 48.2 & 50.8 \\
\hline ESF-HD-140-TEMP8-RTD-11 & -4.56 & 11.89 & -3.84 & 5.96 & 26.7 & 26.8 & 27.5 & 29.5 & 32.6 & 35.8 & 39.1 & 42.7 & 46.0 & 48.5 \\
\hline ESF-HD-140-TEMP8-RTD-12 & -4.79 & 11.89 & -4.04 & 6.27 & 26.4 & 26.4 & 26.9 & 28.6 & 31.3 & 34.2 & 37.3 & 40.8 & 43.9 & 46.3 \\
\hline ESF-HD-140-TEMP8-RTD-13 & -5.02 & 11.88 & -4.23 & 6.56 & 26.2 & 26.3 & 26.6 & 28.0 & 30.4 & 33.0 & 35.9 & 39.1 & 42.1 & 44.4 \\
\hline ESF-HD-140-TEMP8-RTD-14 & -5.25 & 11.88 & -4.42 & 6.86 & 26.0 & 26.1 & 26.4 & 27.5 & 29.6 & 31.9 & 34.6 & 37.6 & 40.4 & 42.7 \\
\hline ESF-HD-140-TEMP8-RTD-15 & -5.48 & 11.88 & -4.62 & 7.17 & 25.8 & 25.8 & 26.1 & 27.0 & 28.8 & 30.9 & 33.3 & 36.2 & 38.8 & 41.0 \\
\hline
\end{tabular}




\begin{tabular}{|c|c|c|c|c|c|c|c|c|c|c|c|c|c|c|}
\hline & \multicolumn{14}{|c|}{ Temperature Data Obtained from the DST } \\
\hline & \multicolumn{14}{|c|}{ Days after startup } \\
\hline & \multicolumn{4}{|c|}{ As Built Locations (m) } & 0 & 7 & 17 & 27 & 38 & 48 & 58 & 69 & 79 & 87 \\
\hline & $\mathbf{x}$ & $\mathbf{y}$ & $\mathbf{z}$ & D & \multicolumn{10}{|c|}{$\left({ }^{\circ} \mathbf{C}\right)$} \\
\hline ESF-HD-140-TEMP8-RTD-16 & -5.71 & 11.88 & -4.81 & 7.47 & 25.5 & 25.6 & 25.8 & 26.5 & 28.1 & 30.0 & 32.2 & 34.9 & 37.4 & 39.4 \\
\hline ESF-HD-140-TEMP8-RTD-17 & -5.94 & 11.88 & -5.01 & 7.77 & 25.3 & 25.4 & 25.6 & 26.2 & 27.5 & 29.2 & 31.2 & 33.6 & 36.0 & 37.9 \\
\hline ESF-HD-140-TEMP8-RTD-18 & -6.17 & 11.88 & -5.20 & 8.07 & 25.1 & 25.1 & 25.3 & 25.8 & 26.9 & 28.5 & 30.3 & 32.6 & 34.8 & 36.6 \\
\hline ESF-HD-140-TEMP8-RTD-19 & -6.41 & 11.87 & -5.40 & 8.38 & 25.0 & 25.1 & 25.2 & 25.6 & 26.5 & 27.8 & 29.5 & 31.6 & 33.6 & 35.3 \\
\hline ESF-HD-140-TEMP8-RTD-20 & -6.64 & 11.87 & -5.59 & 8.68 & 24.8 & 24.8 & 24.9 & 25.3 & 26.1 & 27.3 & 28.8 & 30.7 & 32.6 & 34.2 \\
\hline ESF-HD-140-TEMP8-RTD-21 & -6.87 & 11.87 & -5.79 & 8.98 & 24.7 & 24.8 & 24.9 & 25.1 & 25.8 & 26.8 & 28.1 & 29.8 & 31.6 & 33.1 \\
\hline ESF-HD-140-TEMP8-RTD-22 & -7.10 & 11.87 & -5.98 & 9.28 & 24.6 & 24.7 & 24.7 & 24.9 & 25.5 & 26.4 & 27.6 & 29.1 & 30.8 & 32.2 \\
\hline ESF-HD-140-TEMP8-RTD-23 & -7.33 & 11.87 & -6.18 & 9.59 & 24.4 & 24.4 & 24.5 & 24.7 & 25.1 & 25.9 & 26.9 & 28.3 & 29.8 & 31.1 \\
\hline ESF-HD-140-TEMP8-RTD-24 & -7.56 & 11.86 & -6.37 & 9.89 & 24.4 & 24.5 & 24.5 & 24.7 & 25.1 & 25.7 & 26.6 & 27.9 & 29.3 & 30.5 \\
\hline ESF-HD-140-TEMP8-RTD-25 & -7.79 & 11.86 & -6.56 & 10.18 & 24.3 & 24.0 & 24.3 & 24.5 & 24.8 & 25.4 & 26.2 & 27.3 & 28.6 & 29.6 \\
\hline ESF-HD-140-TEMP8-RTD-26 & -8.02 & 11.86 & -6.76 & 10.49 & 24.3 & 24.3 & 24.4 & 24.5 & 24.7 & 25.2 & 25.9 & 26.9 & 28.1 & 29.1 \\
\hline ESF-HD-140-TEMP8-RTD-27 & -8.25 & 11.86 & -6.95 & 10.79 & 24.2 & 24.2 & 24.3 & 24.4 & 24.6 & 25.0 & 25.6 & 26.5 & 27.5 & 28.5 \\
\hline ESF-HD-140-TEMP8-RTD-28 & -8.48 & 11.86 & -7.15 & 11.09 & 22.9 & 22.9 & 23.1 & 23.2 & 23.3 & 23.7 & 24.2 & 25.0 & 25.9 & 26.6 \\
\hline ESF-HD-140-TEMP8-RTD-29 & -8.71 & 11.86 & -7.34 & 11.39 & 24.1 & 24.1 & 24.2 & 24.2 & 24.4 & 24.6 & 25.1 & 25.8 & 26.6 & 27.3 \\
\hline ESF-HD-140-TEMP8-RTD-30 & -8.94 & 11.85 & -7.54 & 11.70 & 24.1 & 24.1 & 24.1 & 24.2 & 24.3 & 24.5 & 24.9 & 25.6 & 26.3 & 27.0 \\
\hline ESF-HD-140-TEMP8-RTD-31 & -9.17 & 11.85 & -7.73 & 11.99 & 24.0 & 24.0 & 24.1 & 24.1 & 24.2 & 24.4 & 24.8 & 25.3 & 26.0 & 26.6 \\
\hline ESF-HD-140-TEMP8-RTD-32 & -9.40 & 11.85 & -7.93 & 12.30 & 24.0 & 24.0 & 24.0 & 24.1 & 24.2 & 24.3 & 24.6 & 25.1 & 25.7 & 26.2 \\
\hline ESF-HD-140-TEMP8-RTD-33 & -9.63 & 11.85 & -8.12 & 12.60 & 22.8 & 22.2 & 23.3 & 23.7 & 23.8 & 24.1 & 24.4 & 24.9 & 25.4 & 25.9 \\
\hline ESF-HD-140-TEMP8-RTD-34 & -9.86 & 11.85 & -8.32 & 12.90 & 23.9 & 23.9 & 23.8 & 23.9 & 23.9 & 24.1 & 24.3 & 24.6 & 25.0 & 25.4 \\
\hline ESF-HD-140-TEMP8-RTD-35 & -10.09 & 11.85 & -8.51 & 13.20 & 23.9 & 23.9 & 23.9 & 23.9 & 24.0 & 24.1 & 24.2 & 24.5 & 24.9 & 25.3 \\
\hline ESF-HD-140-TEMP8-RTD-36 & -10.32 & 11.84 & -8.70 & 13.50 & 24.3 & 24.3 & 24.4 & 24.4 & 24.4 & 24.5 & 24.6 & 24.9 & 25.2 & 25.5 \\
\hline ESF-HD-140-TEMP8-RTD-37 & -10.55 & 11.84 & -8.90 & 13.80 & 23.4 & 23.5 & 23.8 & 23.9 & 23.9 & 24.0 & 24.1 & 24.3 & 24.6 & 24.9 \\
\hline ESF-HD-140-TEMP8-RTD-38 & -10.78 & 11.84 & -9.09 & 14.10 & 23.8 & 23.7 & 23.6 & 23.6 & 23.6 & 23.7 & 23.7 & 23.9 & 24.1 & 24.4 \\
\hline ESF-HD-140-TEMP8-RTD-39 & -11.01 & 11.84 & -9.29 & 14.41 & 24.0 & 24.1 & 24.2 & 24.2 & 24.2 & 24.2 & 24.2 & 24.4 & 23.9 & 24.5 \\
\hline ESF-HD-140-TEMP8-RTD-40 & -11.24 & 11.84 & -9.48 & 14.70 & 23.8 & 23.8 & 23.8 & 23.8 & 23.9 & 23.9 & 24.0 & 24.1 & 24.3 & 24.4 \\
\hline ESF-HD-140-TEMP8-RTD-41 & -11.47 & 11.84 & -9.68 & 15.01 & 23.7 & 23.6 & 23.6 & 23.5 & 23.5 & 23.5 & 23.6 & 23.7 & 23.8 & 23.9 \\
\hline ESF-HD-140-TEMP8-RTD-42 & -11.70 & 11.83 & -9.87 & 15.31 & 23.8 & 23.8 & 23.8 & 23.8 & 23.8 & 23.8 & 23.9 & 24.0 & 24.1 & 24.2 \\
\hline ESF-HD-140-TEMP8-RTD-43 & -11.93 & 11.83 & -10.07 & 15.61 & 23.7 & 23.7 & 23.8 & 23.8 & 23.8 & 23.8 & 23.8 & 23.9 & 24.0 & 24.1 \\
\hline ESF-HD-140-TEMP8-RTD-44 & -12.16 & 11.83 & -10.26 & 15.91 & 23.7 & 23.7 & 23.7 & 23.7 & 23.7 & 23.7 & 23.8 & 23.8 & 23.9 & 24.0 \\
\hline ESF-HD-140-TEMP8-RTD-45 & -12.39 & 11.83 & -10.46 & 16.22 & 23.7 & 23.7 & 23.7 & 23.7 & 23.7 & 23.8 & 23.8 & 23.8 & 23.9 & 24.0 \\
\hline ESF-HD-140-TEMP8-RTD-46 & -12.62 & 11.83 & -10.65 & 16.51 & 23.9 & 23.9 & 23.9 & 23.9 & 23.8 & 23.9 & 23.9 & 23.9 & 24.0 & 24.0 \\
\hline ESF-HD-140-TEMP8-RTD-47 & -12.85 & 11.83 & -10.84 & 16.81 & 23.7 & 23.6 & 23.5 & 23.5 & 23.5 & 23.5 & 23.5 & 23.5 & 23.5 & 23.6 \\
\hline ESF-HD-140-TEMP8-RTD-48 & -13.08 & 11.82 & -11.04 & 17.12 & 23.6 & 23.5 & 23.5 & 23.4 & 23.2 & 23.1 & 22.9 & 22.7 & 22.6 & 22.5 \\
\hline ESF-HD-140-TEMP8-RTD-49 & -13.31 & 11.82 & -11.23 & 17.42 & 23.7 & 23.7 & 23.7 & 23.7 & 23.7 & 23.7 & 23.7 & 23.7 & 23.8 & 23.8 \\
\hline ESF-HD-140-TEMP8-RTD-50 & -13.54 & 11.82 & -11.43 & 17.72 & 23.7 & 23.5 & 23.5 & 23.4 & 23.4 & 23.4 & 23.4 & 23.4 & 23.4 & 23.4 \\
\hline ESF-HD-140-TEMP8-RTD-51 & -13.77 & 11.82 & -11.62 & 18.02 & 23.7 & 23.5 & 23.5 & 23.5 & 23.5 & 23.4 & 23.4 & 23.4 & 23.4 & 23.4 \\
\hline ESF-HD-140-TEMP8-RTD-52 & -14.00 & 11.82 & -11.82 & 18.32 & 23.7 & 23.7 & 23.7 & 23.7 & 23.7 & 23.8 & 23.8 & 23.8 & 23.8 & 23.8 \\
\hline
\end{tabular}


First Quarter Report of Temperature Measurements During the Heating Phase of the Drift-Scale Test

\begin{tabular}{|c|c|c|c|c|c|c|c|c|c|c|c|c|c|c|}
\hline \multirow{5}{*}{\begin{tabular}{|l} 
ESF-HD-140-TEMP8-RTD-53 \\
\end{tabular}} & \multicolumn{14}{|c|}{ Temperature Data Obtained from the DST } \\
\hline & \multicolumn{14}{|c|}{ Days after startup } \\
\hline & \multicolumn{4}{|c|}{ As Built Locations (m) } & 0 & 7 & 17 & 27 & 38 & 48 & 58 & 69 & 79 & 87 \\
\hline & $\mathbf{x}$ & $\mathbf{y}$ & $\mathbf{z}$ & D & \multicolumn{10}{|c|}{$\left({ }^{\circ} \mathrm{C}\right)$} \\
\hline & -14.23 & 11.81 & -12.01 & 18.62 & 23.7 & 23.7 & 23.7 & 23.7 & 23.7 & 23.7 & 23.7 & 23.8 & 23.8 & 23.8 \\
\hline ESF-HD-140-TEMP8-RTD-54 & -14.46 & 11.81 & -12.21 & 18.93 & 23.7 & 23.5 & 23.1 & 23.3 & 23.2 & 23.4 & 23.6 & 23.7 & 23.7 & 23.8 \\
\hline ESF-HD-140-TEMP8-RTD-55 & -14.69 & 11.81 & -12.40 & 19.22 & 23.6 & 23.5 & 23.5 & 23.5 & 23.4 & 23.4 & 23.4 & 23.4 & 23.4 & 23.4 \\
\hline ESF-HD-140-TEMP8-RTD-56 & -14.92 & 11.81 & -12.60 & 19.53 & 23.7 & 23.7 & 23.7 & 23.7 & 23.7 & 23.7 & 23.7 & 23.7 & 23.7 & 23.7 \\
\hline ESF-HD-140-TEMP8-RTD-57 & -15.15 & 11.81 & -12.79 & 19.83 & 23.7 & 23.7 & 23.7 & 23.7 & 23.7 & 23.7 & 23.7 & 23.7 & 23.7 & 23.8 \\
\hline ESF-HD-140-TEMP8-RTD-58 & -15.38 & 11.81 & -12.98 & 20.13 & 23.7 & 23.6 & 23.5 & 23.5 & 23.5 & 23.4 & 23.4 & 23.4 & 23.4 & 23.4 \\
\hline ESF-HD-140-TEMP8-RTD-59 & -15.61 & 11.80 & -13.18 & 20.43 & 23.7 & 23.7 & 23.7 & 23.7 & 23.7 & 23.7 & 23.7 & 23.7 & 23.7 & 23.7 \\
\hline ESF-HD-140-TEMP8-RTD-60 & -15.84 & 11.80 & -13.37 & 20.73 & 23.7 & 23.8 & 23.9 & 23.9 & 23.9 & 23.9 & 24.0 & 24.0 & 24.0 & 24.0 \\
\hline ESF-HD-140-TEMP8-RTD-61 & -16.07 & 11.80 & -13.57 & 21.03 & 23.7 & 23.7 & 23.7 & 23.7 & 23.7 & 23.7 & 23.7 & 23.7 & 23.7 & 23.7 \\
\hline ESF-HD-140-TEMP8-RTD-62 & -16.30 & 11.80 & -13.76 & 21.33 & 23.7 & 23.7 & 23.8 & 23.8 & 23.8 & 23.8 & 23.8 & 23.8 & 23.9 & 24.0 \\
\hline ESF-HD-140-TEMP8-RTD-63 & -16.53 & 11.80 & -13.96 & 21.64 & 23.7 & 23.6 & 23.5 & 23.5 & 23.4 & 23.4 & 23.4 & 23.4 & 23.4 & 23.4 \\
\hline ESF-HD-140-TEMP8-RTD-64 & -16.76 & 11.80 & -14.15 & 21.93 & 23.7 & 23.7 & 23.7 & 23.7 & 23.7 & 23.7 & 23.7 & 23.7 & 23.7 & 23.7 \\
\hline ESF-HD-140-TEMP8-RTD-65 & -16.99 & 11.79 & -14.35 & 22.24 & 23.7 & 23.8 & 23.9 & 23.9 & 23.7 & 23.9 & 24.0 & 24.1 & 24.0 & 24.1 \\
\hline ESF-HD-140-TEMP8-RTD-66 & -17.22 & 11.79 & -14.54 & 22.54 & 23.7 & 23.7 & 23.7 & 23.7 & 23.7 & 23.7 & 23.7 & 23.7 & 23.7 & 23.7 \\
\hline \multicolumn{15}{|l|}{ ESF-HD-140-TEMP8-RTD-67 } \\
\hline \multicolumn{15}{|l|}{ BH 141 Gage ID } \\
\hline ESF-HD-141-TEMP9-RTD-1 & 0.75 & 11.90 & -3.14 & 3.14 & 29.5 & 30.6 & 35.7 & 40.6 & 45.4 & 49.5 & 53.2 & 57.1 & 60.3 & 62.8 \\
\hline ESF-HD-141-TEMP9-RTD-2 & 0.75 & 11.91 & -3.45 & 3.45 & 29.1 & 29.6 & 33.4 & 37.5 & 41.9 & 45.6 & 49.1 & 52.8 & 55.9 & 58.3 \\
\hline ESF-HD-141-TEMP9-RTD-3 & 0.74 & 11.91 & -3.75 & 3.75 & 28.8 & 28.9 & 31.6 & 35.1 & 38.9 & 42.3 & 45.6 & 49.0 & 52.0 & 54.3 \\
\hline ESF-HD-141-TEMP9-RTD-4 & 0.74 & 11.91 & -4.05 & 4.05 & 28.5 & 28.5 & 30.3 & 33.1 & 36.5 & 39.5 & 42.5 & 45.7 & 48.6 & 50.7 \\
\hline ESF-HD-141-TEMP9-RTD-5 & 0.74 & 11.91 & -4.35 & 4.35 & 27.9 & 27.8 & 29.1 & 31.4 & 34.2 & 36.9 & 39.6 & 42.6 & 45.2 & 47.2 \\
\hline ESF-HD-141-TEMP9-RTD-6 & 0.74 & 11.91 & -4.65 & 4.65 & 27.7 & 27.7 & 28.4 & 30.1 & 32.5 & 34.8 & 37.3 & 40.0 & 42.4 & 44.3 \\
\hline ESF-HD-141-TEMP9-RTD-7 & 0.73 & 11.92 & -4.95 & 4.95 & 27.4 & 27.4 & 27.8 & 29.1 & 31.1 & 33.1 & 35.3 & 37.7 & 40.0 & 41.8 \\
\hline ESF-HD-141-TEMP9-RTD-8 & 0.73 & 11.92 & -5.25 & 5.25 & 27.1 & 27.0 & 27.3 & 28.3 & 29.9 & 31.6 & 33.5 & 35.7 & 37.8 & 39.4 \\
\hline ESF-HD-141-TEMP9-RTD-9 & 0.73 & 11.92 & -5.56 & 5.56 & 26.8 & 26.8 & 26.9 & 27.6 & 28.9 & 30.4 & 32.1 & 34.0 & 35.9 & 37.4 \\
\hline ESF-HD-141-TEMP9-RTD-10 & 0.73 & 11.92 & -5.86 & 5.86 & 26.5 & 26.6 & 26.7 & 27.2 & 28.2 & 29.5 & 30.9 & 32.6 & 34.3 & 35.7 \\
\hline ESF-HD-141-TEMP9-RTD-11 & 0.72 & 11.92 & -6.16 & 6.16 & 25.8 & 26.0 & 26.3 & 26.7 & 27.5 & 28.5 & 29.7 & 31.2 & 32.7 & 33.9 \\
\hline ESF-HD-141-TEMP9-RTD-12 & 0.72 & 11.93 & -6.46 & 6.46 & 26.0 & 26.1 & 26.1 & 26.4 & 27.0 & 27.9 & 28.9 & 30.2 & 31.5 & 32.6 \\
\hline ESF-HD-141-TEMP9-RTD-13 & 0.72 & 11.93 & -6.76 & 6.76 & 25.4 & 25.5 & 25.6 & 26.0 & 25.5 & 26.2 & 27.1 & 29.1 & 29.8 & 31.4 \\
\hline ESF-HD-141-TEMP9-RTD-14 & 0.71 & 11.93 & -7.06 & 7.06 & 25.6 & 25.6 & 25.7 & 25.9 & 26.2 & 26.8 & 27.5 & 28.4 & 29.5 & 30.3 \\
\hline ESF-HD-141-TEMP9-RTD-15 & 0.71 & 11.93 & -7.36 & 7.36 & 25.4 & 25.5 & 25.6 & 25.7 & 26.0 & 26.4 & 27.0 & 27.8 & 28.7 & 29.5 \\
\hline ESF-HD-141-TEMP9-RTD-16 & 0.71 & 11.93 & -7.67 & 7.67 & 25.2 & 25.3 & 25.3 & 25.4 & 25.7 & 26.0 & 26.5 & 27.2 & 27.9 & 28.6 \\
\hline ESF-HD-141-TEMP9-RTD-17 & 0.71 & 11.94 & -7.97 & 7.97 & 25.1 & 25.2 & 25.2 & 25.3 & 25.5 & 25.8 & 26.2 & 26.8 & 27.4 & 28.0 \\
\hline ESF-HD-141-TEMP9-RTD-18 & 0.70 & 11.94 & -8.27 & 8.27 & 24.9 & 25.0 & 25.1 & 25.1 & 25.3 & 25.5 & 25.8 & 26.3 & 26.8 & 27.4 \\
\hline ESF-HD-141-TEMP9-RTD-19 & 0.70 & 11.94 & -8.57 & 8.57 & 24.6 & 24.8 & 24.9 & 25.0 & 25.1 & 25.2 & 25.5 & 25.9 & 26.3 & 26.8 \\
\hline ESF-HD-141-TEMP9-RTD-20 & 0.70 & 11.94 & -8.87 & 8.87 & 24.6 & 24.7 & 24.8 & 24.9 & 25.0 & 25.1 & 25.3 & 25.6 & 26.0 & 26.4 \\
\hline ESF-HD-141-TEMP9-RTD-21 & 0.70 & 11.94 & -9.17 & 9.17 & 24.6 & 24.7 & 24.7 & 24.8 & 24.9 & 25.0 & 25.2 & 25.4 & 25.7 & 26.1 \\
\hline
\end{tabular}




\begin{tabular}{|c|c|c|c|c|c|c|c|c|c|c|c|c|c|c|}
\hline & \multicolumn{14}{|c|}{ Temperature Data Obtained from the DST } \\
\hline & \multicolumn{14}{|c|}{ Days after startup } \\
\hline & \multicolumn{4}{|c|}{ As Built Locations (m) } & 0 & 7 & 17 & 27 & 38 & 48 & 58 & 69 & 79 & 87 \\
\hline & $\mathbf{x}$ & $\mathbf{y}$ & $\mathbf{z}$ & D & \multicolumn{10}{|c|}{$\left({ }^{\circ} \mathbf{C}\right)$} \\
\hline ESF-HD-141-TEMP9-RTD-22 & 0.69 & 11.94 & -9.47 & 9.47 & 23.9 & 24.5 & 24.5 & 24.6 & 24.7 & 24.8 & 24.9 & 25.1 & 25.4 & 25.6 \\
\hline ESF-HD-141-TEMP9-RTD-23 & 0.69 & 11.95 & -9.78 & 9.78 & 24.4 & 24.4 & 24.5 & 24.6 & 24.6 & 24.7 & 24.8 & 25.0 & 25.2 & 25.4 \\
\hline ESF-HD-141-TEMP9-RTD-24 & 0.69 & 11.95 & -10.08 & 10.08 & 24.1 & 23.4 & 23.9 & 24.2 & 24.0 & 24.1 & 24.2 & 24.6 & 24.6 & 25.0 \\
\hline ESF-HD-141-TEMP9-RTD-25 & 0.68 & 11.95 & -10.38 & 10.38 & 24.1 & 24.2 & 24.3 & 24.3 & 24.4 & 24.5 & 24.5 & 24.6 & 24.8 & 24.9 \\
\hline ESF-HD-141-TEMP9-RTD-26 & 0.68 & 11.95 & -10.68 & 10.68 & 24.2 & 24.2 & 24.2 & 24.3 & 24.3 & 24.4 & 24.4 & 24.5 & 24.6 & 24.8 \\
\hline ESF-HD-141-TEMP9-RTD-27 & 0.68 & 11.95 & -10.98 & 10.98 & 24.1 & 24.2 & 24.2 & 24.3 & 24.3 & 24.4 & 24.4 & 24.5 & 24.6 & 24.7 \\
\hline ESF-HD-141-TEMP9-RTD-28 & 0.68 & 11.96 & -11.28 & 11.28 & 24.1 & 24.1 & 24.1 & 24.2 & 24.2 & 24.3 & 24.3 & 24.4 & 24.5 & 24.5 \\
\hline ESF-HD-141-TEMP9-RTD-29 & 0.67 & 11.96 & -11.58 & 11.58 & 24.0 & 24.0 & 24.1 & 24.2 & 24.2 & 24.2 & 24.3 & 24.4 & 24.4 & 24.5 \\
\hline ESF-HD-141-TEMP9-RTD-30 & 0.67 & 11.96 & -11.89 & 11.89 & 23.8 & 23.6 & 23.6 & 23.4 & 23.5 & 23.5 & 23.6 & 23.6 & 23.8 & 23.9 \\
\hline ESF-HD-141-TEMP9-RTD-31 & 0.67 & 11.96 & -12.19 & 12.19 & 24.0 & 24.0 & 24.0 & 24.0 & 24.1 & 24.1 & 24.1 & 24.2 & 24.2 & 24.3 \\
\hline ESF-HD-141-TEMP9-RTD-32 & 0.67 & 11.96 & -12.49 & 12.49 & 24.0 & 24.0 & 24.0 & 24.0 & 24.1 & 24.1 & 24.1 & 24.1 & 24.2 & 24.2 \\
\hline ESF-HD-141-TEMP9-RTD-33 & 0.66 & 11.97 & -12.79 & 12.79 & 23.6 & 23.6 & 23.6 & 23.6 & 23.7 & 23.8 & 23.8 & 23.8 & 23.9 & 23.9 \\
\hline ESF-HD-141-TEMP9-RTD-34 & 0.66 & 11.97 & -13.09 & 13.09 & 23.9 & 23.8 & 23.7 & 23.7 & 23.7 & 23.7 & 23.7 & 23.7 & 23.7 & 23.8 \\
\hline ESF-HD-141-TEMP9-RTD-35 & 0.66 & 11.97 & -13.39 & 13.39 & 23.9 & 23.9 & 24.0 & 24.0 & 24.0 & 24.1 & 24.1 & 24.1 & 24.1 & 24.2 \\
\hline ESF-HD-141-TEMP9-RTD-36 & 0.65 & 11.97 & -13.69 & 13.69 & 23.9 & 23.9 & 24.2 & 24.3 & 24.5 & 35.2 & 24.5 & 24.5 & 24.6 & 24.3 \\
\hline ESF-HD-141-TEMP9-RTD-37 & 0.65 & 11.97 & -14.00 & 14.00 & 23.8 & 23.9 & 23.9 & 23.9 & 23.9 & 24.0 & 24.0 & 24.0 & 24.0 & 24.0 \\
\hline ESF-HD-141-TEMP9-RTD-38 & 0.65 & 11.98 & -14.30 & 14.30 & 23.8 & 23.8 & 23.8 & 23.8 & 23.8 & 23.8 & 23.9 & 23.9 & 23.9 & 23.9 \\
\hline ESF-HD-141-TEMP9-RTD-39 & 0.65 & 11.98 & -14.60 & 14.60 & 23.8 & 23.8 & 23.8 & 23.8 & 23.8 & 23.9 & 23.9 & 23.9 & 23.9 & 23.9 \\
\hline ESF-HD-141-TEMP9-RTD-40 & 0.64 & 11.98 & -14.90 & 14.90 & 24.0 & 24.0 & 24.2 & 24.1 & 24.1 & 24.2 & 24.2 & 24.2 & 24.2 & 23.9 \\
\hline ESF-HD-141-TEMP9-RTD-41 & 0.64 & 11.98 & -15.20 & 15.20 & 24.0 & 24.3 & 24.7 & 24.1 & 24.1 & 24.2 & 24.2 & 24.2 & 24.2 & 23.9 \\
\hline ESF-HD-141-TEMP9-RTD-42 & 0.64 & 11.98 & -15.50 & 15.50 & 23.8 & 23.8 & 23.9 & 23.9 & 23.9 & 23.9 & 23.9 & 23.9 & 23.9 & 23.9 \\
\hline ESF-HD-141-TEMP9-RTD-43 & 0.64 & 11.99 & -15.80 & 15.80 & 23.7 & 23.5 & 23.7 & 23.8 & 23.8 & 23.8 & 23.8 & 23.8 & 23.8 & 23.8 \\
\hline ESF-HD-141-TEMP9-RTD-44 & 0.63 & 11.99 & -16.11 & 16.11 & 23.8 & 23.9 & 23.9 & 23.9 & 23.9 & 23.9 & 23.9 & 23.9 & 23.9 & 24.0 \\
\hline ESF-HD-141-TEMP9-RTD-45 & 0.63 & 11.99 & -16.41 & 16.41 & 23.8 & 23.8 & 23.8 & 23.8 & 23.8 & 23.9 & 23.9 & 23.9 & 23.9 & 23.9 \\
\hline ESF-HD-141-TEMP9-RTD-46 & 0.63 & 11.99 & -16.71 & 16.71 & 23.8 & 23.8 & 23.8 & 23.8 & 23.8 & 23.8 & 23.8 & 23.8 & 23.8 & 23.8 \\
\hline ESF-HD-141-TEMP9-RTD-47 & 0.62 & 11.99 & -17.01 & 17.01 & 23.8 & 23.8 & 23.8 & 23.8 & 23.8 & 23.8 & 23.8 & 23.8 & 23.8 & 23.9 \\
\hline ESF-HD-141-TEMP9-RTD-48 & 0.62 & 12.00 & -17.31 & 17.31 & 23.8 & 23.8 & 23.8 & 23.8 & 23.8 & 23.8 & 23.8 & 23.8 & 23.8 & 23.8 \\
\hline ESF-HD-141-TEMP9-RTD-49 & 0.62 & 12.00 & -17.61 & 17.61 & 23.8 & 23.9 & 24.0 & 24.0 & 24.0 & 24.1 & 24.1 & 24.1 & 24.1 & 24.2 \\
\hline ESF-HD-141-TEMP9-RTD-50 & 0.62 & 12.00 & -17.91 & 17.91 & 23.8 & 23.8 & 23.8 & 23.8 & 23.9 & 23.9 & 23.9 & 23.9 & 23.9 & 23.9 \\
\hline ESF-HD-141-TEMP9-RTD-51 & 0.61 & 12.00 & -18.22 & 18.22 & 23.7 & 23.7 & 23.7 & 23.7 & 23.7 & 23.7 & 23.7 & 23.7 & 23.8 & 23.8 \\
\hline ESF-HD-141-TEMP9-RTD-52 & 0.61 & 12.00 & -18.52 & 18.52 & 23.6 & 23.1 & 23.4 & 23.6 & 23.6 & 23.7 & 23.7 & 23.8 & 23.7 & 23.8 \\
\hline ESF-HD-141-TEMP9-RTD-53 & 0.61 & 12.01 & -18.82 & 18.82 & 23.7 & 23.7 & 23.7 & 23.7 & 23.7 & 23.6 & 23.6 & 23.6 & 23.7 & 23.7 \\
\hline ESF-HD-141-TEMP9-RTD-54 & 0.60 & 12.01 & -19.12 & 19.12 & 23.8 & 23.8 & 23.8 & 23.8 & 23.8 & 23.8 & 23.8 & 23.8 & 23.8 & 23.8 \\
\hline ESF-HD-141-TEMP9-RTD-55 & 0.60 & 12.01 & -19.42 & 19.42 & 23.8 & 23.8 & 23.8 & 23.8 & 23.8 & 23.8 & 23.8 & 23.8 & 23.8 & 23.8 \\
\hline ESF-HD-141-TEMP9-RTD-56 & 0.60 & 12.01 & -19.72 & 19.72 & 23.8 & 23.8 & 23.8 & 23.8 & 23.8 & 23.8 & 23.8 & 23.8 & 23.8 & 23.8 \\
\hline ESF-HD-141-TEMP9-RTD-57 & 0.60 & 12.01 & -20.02 & 20.02 & 23.8 & 23.6 & 23.7 & 23.7 & 23.7 & 23.7 & 23.7 & 23.7 & 23.7 & 23.7 \\
\hline ESF-HD-141-TEMP9-RTD-58 & 0.59 & 12.02 & -20.33 & 20.33 & 23.8 & 23.8 & 23.8 & 23.8 & 23.8 & 23.8 & 23.8 & 23.8 & 23.8 & 23.8 \\
\hline
\end{tabular}


First Quarter Report of Temperature Measurements During the Heating Phase of the Drift-Scale Test

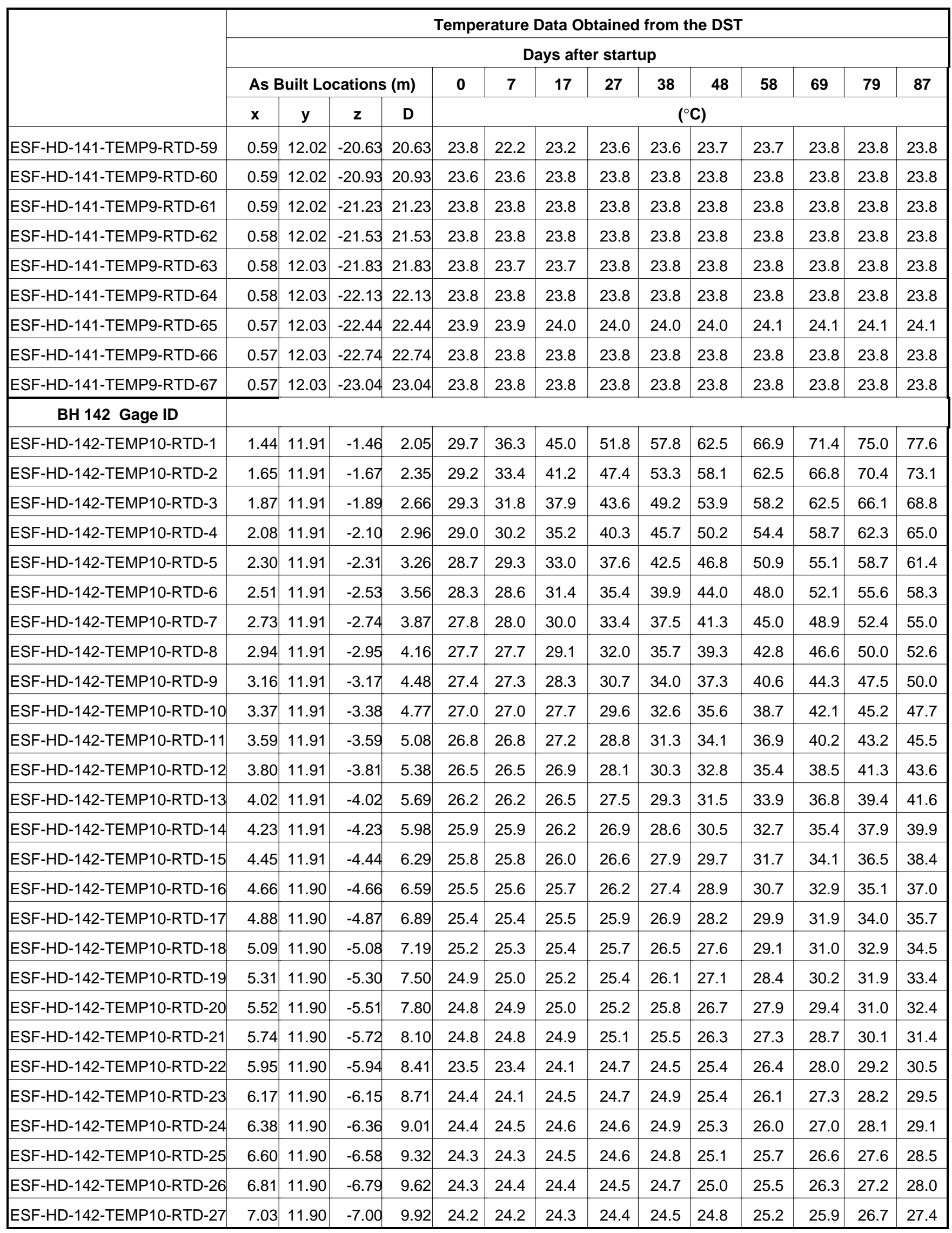




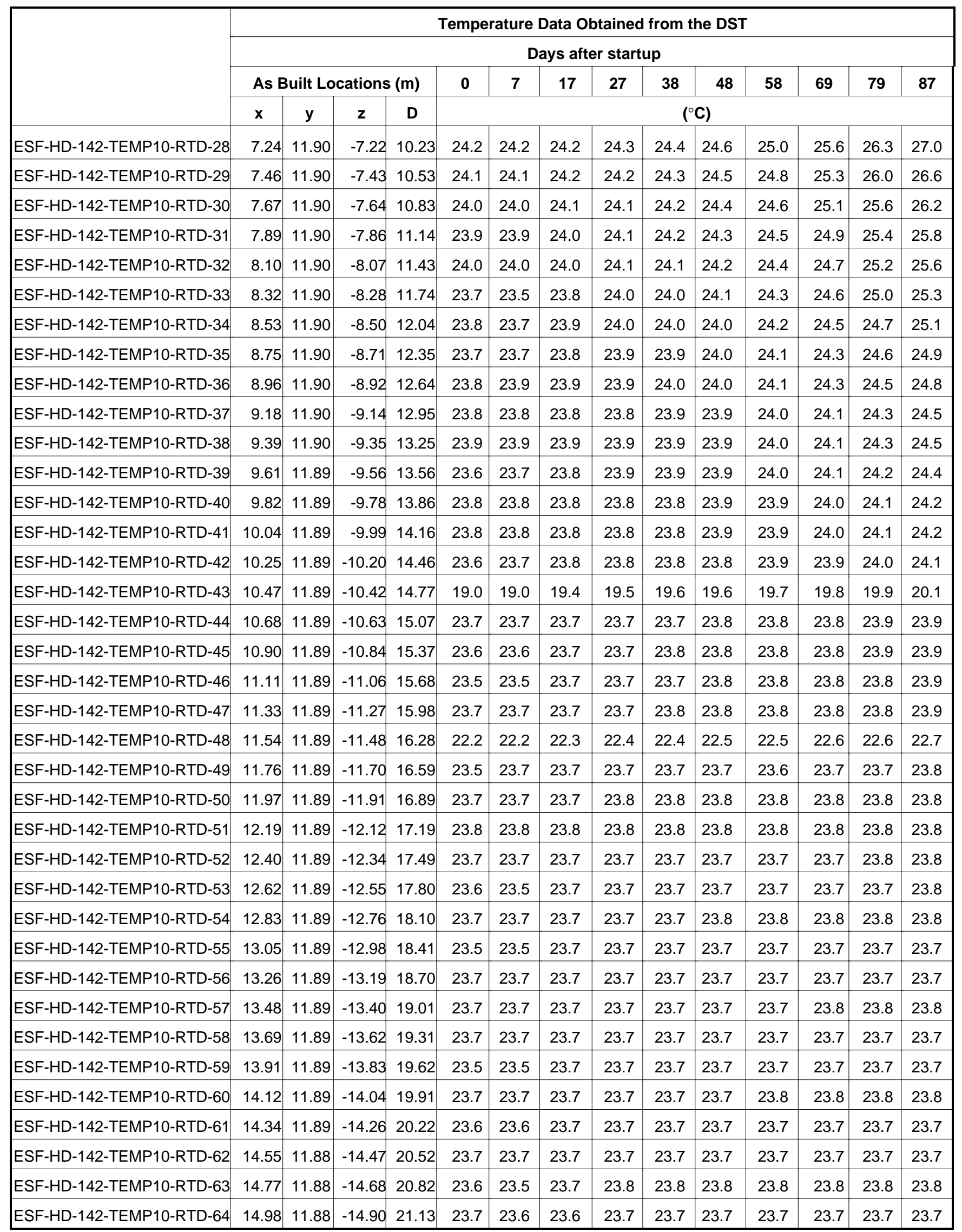


First Quarter Report of Temperature Measurements During the Heating Phase of the Drift-Scale Test

\begin{tabular}{|c|c|c|c|c|c|c|c|c|c|c|c|c|c|c|}
\hline \multirow{5}{*}{\begin{tabular}{|l|} 
\\
ESF-HD-142-TEMP10-RTD-65 \\
\end{tabular}} & \multicolumn{14}{|c|}{ Temperature Data Obtained from the DST } \\
\hline & \multicolumn{14}{|c|}{ Days after startup } \\
\hline & \multicolumn{4}{|c|}{ As Built Locations (m) } & 0 & 7 & 17 & 27 & 38 & 48 & 58 & 69 & 79 & 87 \\
\hline & $\mathbf{x}$ & $\mathbf{y}$ & $\mathbf{z}$ & D & \multicolumn{10}{|c|}{$\left({ }^{\circ} \mathbf{C}\right)$} \\
\hline & 15.20 & 11.88 & -15.11 & 21.43 & 23.6 & 23.4 & 23.7 & 23.7 & 23.7 & 23.7 & 23.8 & 23.8 & 23.8 & 23.8 \\
\hline ESF-HD-142-TEMP10-RTD-66 & 15.41 & 11.88 & -15.32 & 21.73 & 23.7 & 23.6 & 23.7 & 23.7 & 23.7 & 23.7 & 23.7 & 23.7 & 23.8 & 23.8 \\
\hline ESF-HD-142-TEMP10-RTD-67 & 15.63 & 11.88 & -15.54 & 22.04 & 23.3 & 21.4 & 23.1 & 23.6 & 23.6 & 23.7 & 23.7 & 23.7 & 23.7 & 23.7 \\
\hline \multicolumn{15}{|l|}{ BH 143 Gage ID } \\
\hline ESF-HD-143-TEMP11-RTD-1 & 2.39 & 11.89 & -0.01 & 2.39 & 29.9 & 59.4 & 69.4 & 76.3 & 82.9 & 87.8 & 92.1 & 95.9 & 100.3 & 102.2 \\
\hline ESF-HD-143-TEMP11-RTD-2 & 2.68 & 11.89 & -0.01 & 2.68 & 29.8 & 56.0 & 66.5 & 73.7 & 80.6 & 85.5 & 90.0 & 94.0 & 98.2 & 100.3 \\
\hline ESF-HD-143-TEMP11-RTD-3 & 2.98 & 11.89 & -0.01 & 2.98 & 29.6 & 44.2 & 55.9 & 63.6 & 71.4 & 76.8 & 81.5 & 85.9 & 89.8 & 92.6 \\
\hline ESF-HD-143-TEMP11-RTD-4 & 3.28 & 11.89 & -0.01 & 3.28 & 29.5 & 39.9 & 52.0 & 59.9 & 68.1 & 73.6 & 78.6 & 83.2 & 87.2 & 90.3 \\
\hline ESF-HD-143-TEMP11-RTD-5 & 3.58 & 11.89 & -0.01 & 3.58 & 29.2 & 36.7 & 48.8 & 56.7 & 65.4 & 71.0 & 76.1 & 80.9 & 85.2 & 88.4 \\
\hline ESF-HD-143-TEMP11-RTD-6 & 3.88 & 11.89 & -0.01 & 3.88 & 29.1 & 35.7 & 47.5 & 55.5 & 64.5 & 70.2 & 75.6 & 80.3 & 85.1 & 88.0 \\
\hline ESF-HD-143-TEMP11-RTD-7 & 4.18 & 11.89 & -0.01 & 4.18 & 28.7 & 35.4 & 47.1 & 55.2 & 64.3 & 70.4 & 76.0 & 80.8 & 86.4 & 88.6 \\
\hline ESF-HD-143-TEMP11-RTD-8 & 4.48 & 11.89 & -0.01 & 4.48 & 28.5 & 35.9 & 47.6 & 55.9 & 65.1 & 71.5 & 77.3 & 82.0 & 88.7 & 89.7 \\
\hline ESF-HD-143-TEMP11-RTD-9 & 4.78 & 11.89 & -0.01 & 4.78 & 25.4 & 34.5 & 47.7 & 57.3 & 66.5 & 73.4 & 79.5 & 84.4 & 90.1 & 92.1 \\
\hline ESF-HD-143-TEMP11-RTD-10 & 5.08 & 11.89 & 0.00 & 5.08 & 27.8 & 38.1 & 50.4 & 59.4 & 68.6 & 75.4 & 81.4 & 86.5 & 91.7 & 93.8 \\
\hline ESF-HD-143-TEMP11-RTD-11 & 5.38 & 11.89 & 0.00 & 5.38 & 27.5 & 39.2 & 52.1 & 61.5 & 70.6 & 77.4 & 83.4 & 88.7 & 93.8 & 95.2 \\
\hline ESF-HD-143-TEMP11-RTD-12 & 5.68 & 11.89 & 0.00 & 5.68 & 27.2 & 40.1 & 53.5 & 63.1 & 72.3 & 79.0 & 84.8 & 90.2 & 95.0 & 95.9 \\
\hline ESF-HD-143-TEMP11-RTD-13 & 5.98 & 11.89 & 0.00 & 5.98 & 26.8 & 40.8 & 54.8 & 64.7 & 73.8 & 80.3 & 86.0 & 91.0 & 94.9 & 96.1 \\
\hline ESF-HD-143-TEMP11-RTD-14 & 6.27 & 11.89 & 0.00 & 6.27 & 26.6 & 41.0 & 55.5 & 65.6 & 74.7 & 81.2 & 86.8 & 91.2 & 94.9 & 96.1 \\
\hline ESF-HD-143-TEMP11-RTD-15 & 6.57 & 11.90 & 0.00 & 6.57 & 26.3 & 40.4 & 55.3 & 65.7 & 75.0 & 81.6 & 87.2 & 90.8 & 95.0 & 95.7 \\
\hline ESF-HD-143-TEMP11-RTD-16 & 6.87 & 11.90 & 0.00 & 6.87 & 26.1 & 39.7 & 54.7 & 65.2 & 74.6 & 81.4 & 87.1 & 90.3 & 94.6 & 95.0 \\
\hline ESF-HD-143-TEMP11-RTD-17 & 7.17 & 11.90 & 0.00 & 7.17 & 25.8 & 38.8 & 53.9 & 64.6 & 74.0 & 80.8 & 86.4 & 89.9 & 94.5 & 94.8 \\
\hline ESF-HD-143-TEMP11-RTD-18 & 7.47 & 11.90 & 0.00 & 7.47 & 25.6 & 38.4 & 53.5 & 64.4 & 73.7 & 80.5 & 86.2 & 89.4 & 94.8 & 94.3 \\
\hline ESF-HD-143-TEMP11-RTD-19 & 7.77 & 11.90 & 0.00 & 7.77 & 24.4 & 37.3 & 52.6 & 63.6 & 72.8 & 79.7 & 85.5 & 90.2 & 94.9 & 95.2 \\
\hline ESF-HD-143-TEMP11-RTD-20 & 8.07 & 11.90 & 0.00 & 8.07 & 25.3 & 37.2 & 52.0 & 63.0 & 72.2 & 79.2 & 85.1 & 90.3 & 95.0 & 95.6 \\
\hline ESF-HD-143-TEMP11-RTD-21 & 8.37 & 11.90 & 0.00 & 8.37 & 25.1 & 36.2 & 50.9 & 61.8 & 71.0 & 78.0 & 84.0 & 89.5 & 94.6 & 96.1 \\
\hline ESF-HD-143-TEMP11-RTD-22 & 8.67 & 11.90 & 0.00 & 8.67 & 25.0 & 35.4 & 50.0 & 60.9 & 70.1 & 77.2 & 83.2 & 88.8 & 94.0 & 96.1 \\
\hline ESF-HD-143-TEMP11-RTD-23 & 8.97 & 11.90 & 0.00 & 8.97 & 24.8 & 34.9 & 49.7 & 60.5 & 69.7 & 76.9 & 83.0 & 88.7 & 93.7 & 95.8 \\
\hline ESF-HD-143-TEMP11-RTD-24 & 9.27 & 11.90 & 0.00 & 9.27 & 24.6 & 34.6 & 49.5 & 60.4 & 69.6 & 76.8 & 83.1 & 88.6 & 102.9 & 93.5 \\
\hline ESF-HD-143-TEMP11-RTD-25 & 9.57 & 11.90 & 0.00 & 9.57 & 21.9 & 32.2 & 47.4 & 58.8 & 68.5 & 75.5 & 81.5 & 86.4 & 91.1 & 92.2 \\
\hline ESF-HD-143-TEMP11-RTD-26 & 9.86 & 11.90 & 0.00 & 9.86 & 24.5 & 36.0 & 51.4 & 62.9 & 72.3 & 79.6 & 86.0 & 90.5 & 95.3 & 95.2 \\
\hline ESF-HD-143-TEMP11-RTD-27 & 10.16 & 11.90 & 0.00 & 10.16 & 24.4 & 36.9 & 52.8 & 64.6 & 74.0 & 81.4 & 87.8 & 92.4 & 95.8 & 96.3 \\
\hline ESF-HD-143-TEMP11-RTD-28 & 10.46 & 11.90 & 0.00 & 10.46 & 24.2 & 37.3 & 53.5 & 65.5 & 74.9 & 82.2 & 88.4 & 93.0 & 95.8 & 96.4 \\
\hline ESF-HD-143-TEMP11-RTD-29 & 10.76 & 11.90 & 0.00 & 10.76 & 24.2 & 37.5 & 54.1 & 66.4 & 75.8 & 83.2 & 89.4 & 93.5 & 96.1 & 96.6 \\
\hline ESF-HD-143-TEMP11-RTD-30 & 11.06 & 11.90 & 0.00 & 11.06 & 24.2 & 37.8 & 54.6 & 66.8 & 76.1 & 83.4 & 89.6 & 94.1 & 96.2 & 96.8 \\
\hline ESF-HD-143-TEMP11-RTD-31 & 11.36 & 11.90 & 0.01 & 11.36 & 24.1 & 34.2 & 54.1 & 66.6 & 75.3 & 82.9 & 89.0 & 93.3 & 96.1 & 96.8 \\
\hline ESF-HD-143-TEMP11-RTD-32 & 11.66 & 11.90 & 0.01 & 11.66 & 24.1 & 37.8 & 54.6 & 66.6 & 75.5 & 82.6 & 88.4 & 92.4 & 96.1 & 96.5 \\
\hline ESF-HD-143-TEMP11-RTD-33 & 11.96 & 11.90 & 0.01 & 11.96 & 23.9 & 37.5 & 54.0 & 65.6 & 74.1 & 81.0 & 86.6 & 90.7 & 94.5 & 96.0 \\
\hline
\end{tabular}




\begin{tabular}{|c|c|c|c|c|c|c|c|c|c|c|c|c|c|c|}
\hline & \multicolumn{14}{|c|}{ Temperature Data Obtained from the DST } \\
\hline & \multicolumn{14}{|c|}{ Days after startup } \\
\hline & \multicolumn{4}{|c|}{ As Built Locations (m) } & 0 & 7 & 17 & 27 & 38 & 48 & 58 & 69 & 79 & 87 \\
\hline & $\mathbf{x}$ & $\mathbf{y}$ & $\mathbf{z}$ & D & \multicolumn{10}{|c|}{$\left({ }^{\circ} \mathbf{C}\right)$} \\
\hline ESF-HD-143-TEMP11-RTD-34 & 12.26 & 11.90 & 0.01 & 12.26 & 23.9 & 37.3 & 53.3 & 64.7 & 72.7 & 79.5 & 84.9 & 89.6 & 93.2 & 95.5 \\
\hline ESF-HD-143-TEMP11-RTD-35 & 12.56 & 11.90 & 0.01 & 12.56 & 23.8 & 36.4 & 51.6 & 62.6 & 70.1 & 76.5 & 81.8 & 86.5 & 90.1 & 93.4 \\
\hline ESF-HD-143-TEMP11-RTD-36 & 12.86 & 11.90 & 0.01 & 12.86 & 23.9 & 36.3 & 50.5 & 61.1 & 68.2 & 74.4 & 79.4 & 84.2 & 87.7 & 91.0 \\
\hline ESF-HD-143-TEMP11-RTD-37 & 13.16 & 11.90 & 0.01 & 13.16 & 23.8 & 34.5 & 47.5 & 57.2 & 63.9 & 69.8 & 74.6 & 79.1 & 82.5 & 85.5 \\
\hline ESF-HD-143-TEMP11-RTD-38 & 13.45 & 11.91 & 0.01 & 13.45 & 23.8 & 33.6 & 45.5 & 54.4 & 60.8 & 66.4 & 71.0 & 75.3 & 78.6 & 81.3 \\
\hline ESF-HD-143-TEMP11-RTD-39 & 13.75 & 11.91 & 0.01 & 13.75 & 23.7 & 31.2 & 41.4 & 49.1 & 55.1 & 60.3 & 64.6 & 68.5 & 71.8 & 74.3 \\
\hline ESF-HD-143-TEMP11-RTD-40 & 14.05 & 11.91 & 0.01 & 14.05 & 23.6 & 29.2 & 36.3 & 45.3 & 50.2 & 55.1 & 58.2 & 61.9 & 67.8 & 68.3 \\
\hline ESF-HD-143-TEMP11-RTD-41 & 14.35 & 11.91 & 0.01 & 14.35 & 23.6 & 27.3 & 34.5 & 40.2 & 45.4 & 49.6 & 53.5 & 56.9 & 59.9 & 62.2 \\
\hline ESF-HD-143-TEMP11-RTD-42 & 14.65 & 11.91 & 0.01 & 14.65 & 23.7 & 26.3 & 32.2 & 37.2 & 42.0 & 45.9 & 49.5 & 52.7 & 55.7 & 56.5 \\
\hline ESF-HD-143-TEMP11-RTD-43 & 14.95 & 11.91 & 0.01 & 14.95 & 23.7 & 25.0 & 29.3 & 33.4 & 37.5 & 40.9 & 44.1 & 47.2 & 49.9 & 51.9 \\
\hline ESF-HD-143-TEMP11-RTD-44 & 15.25 & 11.91 & 0.01 & 15.25 & 23.7 & 24.5 & 27.8 & 31.3 & 35.0 & 38.1 & 41.1 & 43.9 & 46.5 & 48.4 \\
\hline ESF-HD-143-TEMP11-RTD-45 & 15.55 & 11.91 & 0.01 & 15.55 & 23.5 & 23.7 & 26.0 & 28.8 & 31.8 & 34.5 & 37.0 & 39.7 & 42.0 & 43.7 \\
\hline ESF-HD-143-TEMP11-RTD-46 & 15.85 & 11.91 & 0.01 & 15.85 & 23.6 & 23.8 & 25.5 & 27.8 & 30.5 & 32.8 & 35.2 & 37.6 & 39.8 & 41.4 \\
\hline ESF-HD-143-TEMP11-RTD-47 & 16.15 & 11.91 & 0.01 & 16.15 & 23.6 & 23.7 & 24.7 & 26.4 & 28.6 & 30.6 & 32.6 & 34.8 & 36.7 & 38.1 \\
\hline ESF-HD-143-TEMP11-RTD-48 & 16.45 & 11.91 & 0.01 & 16.45 & 23.2 & 23.5 & 24.3 & 25.7 & 27.6 & 29.3 & 31.1 & 33.1 & 34.8 & 36.2 \\
\hline ESF-HD-143-TEMP11-RTD-49 & 16.75 & 11.91 & 0.01 & 16.75 & 23.6 & 23.6 & 24.0 & 25.0 & 26.4 & 27.8 & 29.4 & 31.1 & 32.6 & 33.8 \\
\hline ESF-HD-143-TEMP11-RTD-50 & 17.04 & 11.91 & 0.01 & 17.04 & 23.6 & 23.6 & 23.9 & 24.6 & 25.8 & 27.0 & 28.4 & 29.9 & 31.3 & 32.4 \\
\hline ESF-HD-143-TEMP11-RTD-51 & 17.34 & 11.91 & 0.01 & 17.34 & 23.6 & 23.6 & 23.7 & 24.2 & 25.1 & 26.1 & 27.2 & 28.5 & 29.7 & 30.7 \\
\hline ESF-HD-143-TEMP11-RTD-52 & 17.64 & 11.91 & 0.01 & 17.64 & 23.6 & 23.6 & 23.7 & 24.0 & 24.8 & 25.6 & 26.6 & 27.7 & 28.8 & 29.7 \\
\hline ESF-HD-143-TEMP11-RTD-53 & 17.94 & 11.91 & 0.02 & 17.94 & 23.6 & 23.6 & 23.6 & 23.9 & 24.4 & 25.0 & 25.8 & 26.8 & 27.7 & 28.5 \\
\hline ESF-HD-143-TEMP11-RTD-54 & 18.24 & 11.91 & 0.02 & 18.24 & 23.6 & 23.6 & 23.6 & 23.8 & 24.2 & 24.7 & 25.4 & 26.3 & 27.1 & 27.8 \\
\hline ESF-HD-143-TEMP11-RTD-55 & 18.54 & 11.91 & 0.02 & 18.54 & 23.0 & 23.0 & 23.0 & 23.1 & 23.4 & 23.8 & 24.4 & 25.1 & 25.8 & 26.3 \\
\hline ESF-HD-143-TEMP11-RTD-56 & 18.84 & 11.91 & 0.02 & 18.84 & 23.5 & 23.5 & 23.6 & 23.6 & 23.9 & 24.2 & 24.6 & 25.2 & 25.8 & 26.3 \\
\hline ESF-HD-143-TEMP11-RTD-57 & 19.14 & 11.91 & 0.02 & 19.14 & 23.4 & 23.4 & 23.5 & 23.6 & 23.7 & 24.0 & 24.3 & 24.8 & 25.3 & 25.7 \\
\hline ESF-HD-143-TEMP11-RTD-58 & 19.44 & 11.91 & 0.02 & 19.44 & 23.6 & 23.5 & 23.6 & 23.6 & 23.7 & 23.9 & 24.2 & 24.6 & 25.0 & 25.4 \\
\hline ESF-HD-143-TEMP11-RTD-59 & 19.74 & 11.91 & 0.02 & 19.74 & 23.5 & 23.6 & 23.6 & 23.6 & 23.7 & 23.8 & 24.0 & 24.3 & 24.7 & 25.0 \\
\hline ESF-HD-143-TEMP11-RTD-60 & 20.04 & 11.91 & 0.02 & 20.04 & 23.5 & 23.5 & 23.6 & 23.6 & 23.6 & 23.7 & 23.9 & 24.2 & 24.5 & 24.8 \\
\hline ESF-HD-143-TEMP11-RTD-61 & 20.34 & 11.91 & 0.02 & 20.34 & 23.6 & 23.6 & 23.6 & 23.6 & 23.6 & 23.7 & 23.8 & 24.0 & 24.3 & 24.5 \\
\hline ESF-HD-143-TEMP11-RTD-62 & 20.63 & 11.92 & 0.02 & 20.63 & 23.5 & 23.5 & 23.5 & 23.5 & 23.6 & 23.6 & 23.7 & 23.9 & 24.1 & 24.3 \\
\hline ESF-HD-143-TEMP11-RTD-63 & 20.93 & 11.92 & 0.02 & 20.93 & 23.4 & 23.4 & 23.5 & 23.5 & 23.6 & 23.6 & 23.7 & 23.8 & 24.0 & 24.2 \\
\hline ESF-HD-143-TEMP11-RTD-64 & 21.23 & 11.92 & 0.02 & 21.23 & 23.3 & 23.3 & 23.5 & 23.5 & 23.5 & 23.4 & 23.6 & 23.7 & 23.8 & 24.0 \\
\hline ESF-HD-143-TEMP11-RTD-65 & 21.53 & 11.92 & 0.02 & 21.53 & 23.4 & 23.5 & 23.5 & 23.6 & 23.6 & 23.6 & 23.6 & 23.7 & 23.8 & 23.9 \\
\hline ESF-HD-143-TEMP11-RTD-66 & 21.83 & 11.92 & 0.02 & 21.83 & 23.5 & 23.5 & 23.5 & 23.5 & 23.5 & 23.6 & 23.6 & 23.7 & 23.8 & 23.9 \\
\hline ESF-HD-143-TEMP11-RTD-67 & 22.13 & 11.92 & 0.02 & 22.13 & 23.6 & 23.5 & 23.7 & 23.9 & 23.6 & 23.9 & 23.5 & 23.9 & 23.9 & 23.9 \\
\hline \multicolumn{15}{|l|}{ BH 144 Gage ID } \\
\hline ESF-HD-144-TEMP12-RTD-1 & 2.07 & 11.92 & 2.04 & 2.91 & 29.6 & 50.6 & 60.6 & 67.5 & 73.9 & 78.7 & 83.1 & 87.1 & 91.0 & 93.1 \\
\hline ESF-HD-144-TEMP12-RTD-2 & 2.28 & 11.92 & 2.25 & 3.20 & 29.7 & 42.5 & 51.9 & 58.6 & 64.8 & 69.8 & 74.2 & 78.3 & 81.9 & 84.4 \\
\hline
\end{tabular}


First Quarter Report of Temperature Measurements During the Heating Phase of the Drift-Scale Test

\begin{tabular}{|c|c|c|c|c|c|c|c|c|c|c|c|c|c|c|}
\hline \multirow{5}{*}{\begin{tabular}{|l|} 
ESF-HD-144-TEMP12-RTD-3 \\
\end{tabular}} & \multicolumn{14}{|c|}{ Temperature Data Obtained from the DST } \\
\hline & \multicolumn{14}{|c|}{ Days after startup } \\
\hline & \multicolumn{4}{|c|}{ As Built Locations (m) } & 0 & 7 & 17 & 27 & 38 & 48 & 58 & 69 & 79 & 87 \\
\hline & $\mathbf{x}$ & $\mathbf{y}$ & $\mathbf{z}$ & D & \multicolumn{10}{|c|}{$\left({ }^{\circ} \mathbf{C}\right)$} \\
\hline & 2.49 & 11.92 & 2.46 & 3.50 & 29.6 & 36.8 & 45.3 & 51.7 & 57.7 & 62.6 & 67.0 & 71.3 & 74.9 & 77.6 \\
\hline ESF-HD-144-TEMP12-RTD-4 & 2.70 & 11.92 & 2.67 & 3.80 & 29.4 & 33.4 & 40.6 & 46.6 & 52.3 & 57.0 & 61.4 & 65.8 & 69.4 & 72.2 \\
\hline ESF-HD-144-TEMP12-RTD-5 & 2.91 & 11.92 & 2.89 & 4.10 & 29.1 & 31.2 & 37.0 & 42.4 & 47.8 & 52.3 & 56.5 & 60.8 & 64.5 & 67.3 \\
\hline ESF-HD-144-TEMP12-RTD-6 & 3.13 & 11.93 & 3.10 & 4.41 & 28.8 & 29.7 & 34.3 & 39.1 & 44.0 & 48.3 & 52.4 & 56.6 & 60.2 & 63.0 \\
\hline ESF-HD-144-TEMP12-RTD-7 & 3.34 & 11.93 & 3.31 & 4.70 & 28.5 & 28.9 & 32.2 & 36.3 & 40.8 & 44.9 & 48.7 & 52.8 & 56.3 & 59.0 \\
\hline ESF-HD-144-TEMP12-RTD-8 & 3.55 & 11.93 & 3.52 & 5.00 & 28.2 & 28.3 & 30.7 & 34.1 & 38.2 & 41.9 & 45.5 & 49.5 & 52.9 & 55.5 \\
\hline ESF-HD-144-TEMP12-RTD-9 & 3.76 & 11.93 & 3.73 & 5.30 & 27.8 & 27.8 & 29.5 & 32.3 & 35.9 & 39.3 & 42.7 & 46.4 & 49.7 & 52.3 \\
\hline ESF-HD-144-TEMP12-RTD-10 & 3.97 & 11.94 & 3.94 & 5.59 & 27.5 & 27.5 & 28.6 & 30.8 & 33.9 & 37.0 & 40.2 & 43.7 & 46.8 & 49.3 \\
\hline ESF-HD-144-TEMP12-RTD-11 & 4.19 & 11.94 & 4.16 & 5.90 & 26.5 & 26.6 & 27.7 & 29.6 & 32.2 & 35.0 & 37.9 & 41.2 & 44.1 & 46.5 \\
\hline ESF-HD-144-TEMP12-RTD-12 & 4.40 & 11.94 & 4.37 & 6.20 & 26.9 & 26.9 & 27.3 & 28.7 & 30.9 & 33.3 & 36.0 & 39.0 & 41.8 & 44.1 \\
\hline ESF-HD-144-TEMP12-RTD-13 & 4.61 & 11.94 & 4.58 & 6.50 & 26.5 & 26.5 & 26.8 & 27.9 & 29.7 & 31.9 & 34.3 & 37.1 & 39.7 & 41.8 \\
\hline ESF-HD-144-TEMP12-RTD-14 & 4.82 & 11.94 & 4.79 & 6.80 & 26.2 & 25.7 & 26.3 & 27.2 & 28.5 & 30.3 & 32.5 & 35.4 & 37.2 & 39.7 \\
\hline ESF-HD-144-TEMP12-RTD-15 & 5.03 & 11.95 & 5.00 & 7.09 & 19.5 & 21.1 & 25.0 & 26.7 & 28.0 & 29.6 & 31.6 & 33.9 & 36.2 & 38.1 \\
\hline ESF-HD-144-TEMP12-RTD-16 & 5.25 & 11.95 & 5.21 & 7.40 & 25.8 & 25.8 & 25.9 & 26.4 & 27.4 & 28.8 & 30.5 & 32.6 & 34.7 & 36.4 \\
\hline ESF-HD-144-TEMP12-RTD-17 & 5.46 & 11.95 & 5.43 & 7.70 & 25.6 & 25.6 & 25.7 & 26.1 & 26.9 & 28.1 & 29.6 & 31.5 & 33.4 & 35.0 \\
\hline ESF-HD-144-TEMP12-RTD-18 & 5.67 & 11.95 & 5.64 & 8.00 & 25.4 & 25.4 & 25.5 & 25.7 & 26.4 & 27.4 & 28.7 & 30.4 & 32.1 & 33.6 \\
\hline ESF-HD-144-TEMP12-RTD-19 & 5.88 & 11.95 & 5.85 & 8.29 & 25.2 & 25.2 & 25.3 & 25.5 & 26.0 & 26.8 & 28.0 & 29.5 & 31.1 & 32.4 \\
\hline ESF-HD-144-TEMP12-RTD-20 & 6.09 & 11.96 & 6.06 & 8.59 & 24.8 & 25.0 & 25.1 & 25.2 & 25.7 & 26.4 & 27.3 & 28.7 & 30.1 & 31.3 \\
\hline ESF-HD-144-TEMP12-RTD-21 & 6.31 & 11.96 & 6.27 & 8.90 & 24.7 & 24.8 & 25.0 & 25.1 & 25.4 & 26.0 & 26.8 & 28.0 & 29.3 & 30.4 \\
\hline ESF-HD-144-TEMP12-RTD-22 & 6.52 & 11.96 & 6.49 & 9.20 & 24.5 & 24.6 & 24.8 & 24.9 & 25.1 & 25.6 & 26.3 & 27.4 & 28.5 & 29.5 \\
\hline ESF-HD-144-TEMP12-RTD-23 & 6.73 & 11.96 & 6.70 & 9.50 & 24.5 & 24.6 & 24.7 & 24.8 & 25.0 & 25.4 & 26.0 & 26.9 & 27.9 & 28.8 \\
\hline ESF-HD-144-TEMP12-RTD-24 & 6.94 & 11.97 & 6.91 & 9.79 & 24.4 & 24.5 & 24.6 & 24.6 & 24.8 & 25.1 & 25.6 & 26.4 & 27.3 & 28.1 \\
\hline ESF-HD-144-TEMP12-RTD-25 & 7.15 & 11.97 & 7.12 & 10.09 & 24.3 & 24.3 & 24.4 & 24.5 & 24.6 & 24.9 & 25.3 & 26.0 & 26.7 & 27.5 \\
\hline ESF-HD-144-TEMP12-RTD-26 & 7.37 & 11.97 & 7.33 & 10.40 & 24.2 & 24.2 & 24.3 & 24.4 & 24.5 & 24.7 & 25.1 & 25.6 & 26.3 & 27.0 \\
\hline ESF-HD-144-TEMP12-RTD-27 & 7.58 & 11.97 & 7.54 & 10.69 & 24.1 & 24.2 & 24.2 & 24.3 & 24.4 & 24.6 & 24.9 & 25.3 & 25.9 & 26.5 \\
\hline ESF-HD-144-TEMP12-RTD-28 & 7.79 & 11.97 & 7.76 & 11.00 & 24.0 & 24.1 & 24.2 & 24.2 & 24.3 & 24.4 & 24.7 & 25.1 & 25.6 & 26.1 \\
\hline ESF-HD-144-TEMP12-RTD-29 & 8.00 & 11.98 & 7.97 & 11.29 & 24.0 & 24.0 & 24.1 & 24.1 & 24.2 & 24.3 & 24.5 & 24.9 & 25.3 & 25.7 \\
\hline ESF-HD-144-TEMP12-RTD-30 & 8.21 & 11.98 & 8.18 & 11.59 & 23.6 & 23.8 & 23.9 & 24.0 & 24.0 & 24.1 & 24.3 & 24.6 & 25.0 & 25.4 \\
\hline ESF-HD-144-TEMP12-RTD-31 & 8.43 & 11.98 & 8.39 & 11.89 & 20.6 & 23.4 & 23.9 & 24.0 & 23.8 & 23.2 & 23.7 & 24.5 & 24.6 & 25.2 \\
\hline ESF-HD-144-TEMP12-RTD-32 & 8.64 & 11.98 & 8.60 & 12.19 & 23.5 & 23.5 & 23.5 & 23.6 & 23.7 & 23.7 & 23.9 & 24.1 & 24.4 & 24.7 \\
\hline ESF-HD-144-TEMP12-RTD-33 & 8.85 & 11.99 & 8.81 & 12.49 & 23.7 & 27.7 & 25.5 & 26.2 & 25.6 & 24.0 & 24.3 & 24.1 & 24.3 & 25.4 \\
\hline ESF-HD-144-TEMP12-RTD-34 & 9.06 & 11.99 & 9.03 & 12.79 & 23.6 & 23.7 & 23.7 & 23.8 & 23.8 & 23.9 & 24.0 & 24.1 & 24.3 & 24.5 \\
\hline ESF-HD-144-TEMP12-RTD-35 & 9.27 & 11.99 & 9.24 & 13.09 & 23.7 & 23.7 & 23.8 & 23.8 & 23.8 & 23.9 & 23.9 & 24.1 & 24.2 & 24.4 \\
\hline ESF-HD-144-TEMP12-RTD-36 & 9.48 & 11.99 & 9.45 & 13.39 & 23.5 & 23.5 & 23.6 & 23.7 & 23.7 & 23.8 & 23.8 & 23.9 & 24.1 & 24.2 \\
\hline ESF-HD-144-TEMP12-RTD-37 & 9.70 & 11.99 & 9.66 & 13.69 & 23.3 & 23.3 & 23.4 & 23.4 & 23.5 & 23.5 & 23.6 & 23.7 & 23.9 & 24.0 \\
\hline ESF-HD-144-TEMP12-RTD-38 & 9.91 & 12.00 & 9.87 & 13.99 & 23.4 & 23.4 & 23.6 & 23.6 & 23.7 & 23.7 & 23.7 & 23.8 & 23.9 & 24.0 \\
\hline ESF-HD-144-TEMP12-RTD-39 & 10.12 & 12.00 & 10.08 & 14.28 & 23.3 & 23.1 & 23.5 & 23.5 & 23.6 & 23.6 & 23.6 & 23.7 & 23.8 & 23.9 \\
\hline
\end{tabular}




\begin{tabular}{|c|c|c|c|c|c|c|c|c|c|c|c|c|c|c|}
\hline & \multicolumn{14}{|c|}{ Temperature Data Obtained from the DST } \\
\hline & \multicolumn{14}{|c|}{ Days after startup } \\
\hline & \multicolumn{4}{|c|}{ As Built Locations (m) } & 0 & 7 & 17 & 27 & 38 & 48 & 58 & 69 & 79 & 87 \\
\hline & $\mathbf{x}$ & $\mathbf{y}$ & $\mathbf{z}$ & D & \multicolumn{10}{|c|}{$\left({ }^{\circ} \mathbf{C}\right)$} \\
\hline ESF-HD-144-TEMP12-RTD-40 & 10.33 & 12.00 & 10.30 & 14.59 & 23.4 & 23.5 & 23.5 & 23.5 & 23.5 & 23.6 & 23.6 & 23.7 & 23.7 & 23.8 \\
\hline ESF-HD-144-TEMP12-RTD-41 & 10.54 & 12.00 & 10.51 & 14.89 & 23.3 & 23.2 & 23.4 & 23.5 & 23.5 & 23.6 & 23.6 & 23.7 & 23.7 & 23.8 \\
\hline ESF-HD-144-TEMP12-RTD-42 & 10.76 & 12.00 & 10.72 & 15.19 & 23.5 & 23.5 & 23.5 & 23.5 & 23.5 & 23.5 & 23.5 & 23.6 & 23.6 & 23.7 \\
\hline ESF-HD-144-TEMP12-RTD-43 & 10.97 & 12.01 & 10.93 & 15.49 & 23.3 & 23.3 & 23.4 & 23.5 & 23.5 & 23.5 & 23.5 & 23.5 & 23.6 & 23.6 \\
\hline ESF-HD-144-TEMP12-RTD-44 & 11.18 & 12.01 & 11.14 & 15.78 & 23.2 & 23.2 & 23.4 & 23.4 & 23.4 & 23.5 & 23.5 & 23.5 & 23.5 & 23.6 \\
\hline ESF-HD-144-TEMP12-RTD-45 & 11.39 & 12.01 & 11.36 & 16.09 & 23.4 & 23.4 & 23.4 & 23.4 & 23.5 & 23.5 & 23.5 & 23.5 & 23.5 & 23.6 \\
\hline ESF-HD-144-TEMP12-RTD-46 & 11.60 & 12.01 & 11.57 & 16.38 & 23.4 & 23.6 & 23.6 & 23.7 & 23.7 & 23.7 & 23.8 & 23.8 & 23.9 & 23.9 \\
\hline ESF-HD-144-TEMP12-RTD-47 & 11.82 & 12.02 & 11.78 & 16.69 & 23.4 & 23.4 & 23.4 & 23.4 & 23.4 & 23.4 & 23.4 & 23.4 & 23.5 & 23.5 \\
\hline ESF-HD-144-TEMP12-RTD-48 & 12.03 & 12.02 & 11.99 & 16.99 & 23.4 & 23.4 & 23.4 & 23.4 & 23.4 & 23.4 & 23.4 & 23.4 & 23.4 & 23.4 \\
\hline ESF-HD-144-TEMP12-RTD-49 & 12.24 & 12.02 & 12.20 & 17.28 & 23.4 & 23.4 & 23.4 & 23.4 & 23.4 & 23.4 & 23.4 & 23.4 & 23.5 & 23.5 \\
\hline ESF-HD-144-TEMP12-RTD-50 & 12.45 & 12.02 & 12.41 & 17.58 & 23.1 & 23.2 & 23.4 & 23.4 & 23.4 & 23.4 & 23.4 & 23.4 & 23.4 & 23.4 \\
\hline ESF-HD-144-TEMP12-RTD-51 & 12.66 & 12.02 & 12.63 & 17.88 & 23.4 & 23.4 & 23.4 & 23.4 & 23.4 & 23.4 & 23.4 & 23.4 & 23.4 & 23.5 \\
\hline ESF-HD-144-TEMP12-RTD-52 & 12.88 & 12.03 & 12.84 & 18.19 & 23.3 & 23.2 & 23.4 & 23.6 & 23.6 & 23.6 & 23.7 & 23.7 & 23.7 & 23.7 \\
\hline ESF-HD-144-TEMP12-RTD-53 & 13.09 & 12.03 & 13.05 & 18.48 & 23.1 & 23.1 & 23.3 & 23.4 & 23.4 & 23.4 & 23.4 & 23.4 & 23.4 & 23.4 \\
\hline ESF-HD-144-TEMP12-RTD-54 & 13.30 & 12.03 & 13.26 & 18.78 & 23.4 & 23.4 & 23.4 & 23.4 & 23.4 & 23.4 & 23.4 & 23.4 & 23.4 & 23.4 \\
\hline ESF-HD-144-TEMP12-RTD-55 & 13.51 & 12.03 & 13.47 & 19.08 & 23.3 & 23.4 & 23.4 & 23.4 & 23.4 & 23.4 & 23.4 & 23.4 & 23.4 & 23.4 \\
\hline ESF-HD-144-TEMP12-RTD-56 & 13.72 & 12.04 & 13.68 & 19.38 & 23.3 & 23.3 & 23.3 & 23.3 & 23.3 & 23.3 & 23.3 & 23.3 & 23.3 & 23.3 \\
\hline ESF-HD-144-TEMP12-RTD-57 & 13.94 & 12.04 & 13.90 & 19.69 & 22.5 & 23.3 & 23.3 & 23.3 & 23.3 & 23.3 & 23.4 & 23.4 & 23.4 & 23.4 \\
\hline ESF-HD-144-TEMP12-RTD-58 & 14.15 & 12.04 & 14.11 & 19.98 & 23.2 & 23.3 & 23.3 & 23.3 & 23.3 & 23.3 & 23.3 & 23.3 & 23.3 & 23.3 \\
\hline ESF-HD-144-TEMP12-RTD-59 & 14.36 & 12.04 & 14.32 & 20.28 & 23.3 & 23.3 & 23.3 & 23.3 & 23.3 & 23.3 & 23.3 & 23.3 & 23.3 & 23.4 \\
\hline ESF-HD-144-TEMP12-RTD-60 & 14.57 & 12.04 & 14.53 & 20.58 & 23.3 & 23.3 & 23.3 & 23.3 & 23.3 & 23.3 & 23.4 & 23.4 & 23.4 & 23.4 \\
\hline ESF-HD-144-TEMP12-RTD-61 & 14.78 & 12.05 & 14.74 & 20.87 & 22.4 & 23.2 & 23.3 & 23.3 & 23.3 & 23.3 & 23.3 & 23.3 & 23.3 & 23.3 \\
\hline ESF-HD-144-TEMP12-RTD-62 & 15.00 & 12.05 & 14.96 & 21.19 & 23.2 & 23.2 & 23.2 & 23.3 & 23.3 & 23.3 & 23.3 & 23.3 & 23.2 & 23.3 \\
\hline ESF-HD-144-TEMP12-RTD-63 & 15.21 & 12.05 & 15.17 & 21.48 & 23.3 & 23.3 & 23.3 & 23.3 & 23.3 & 23.3 & 23.3 & 23.3 & 23.3 & 23.3 \\
\hline ESF-HD-144-TEMP12-RTD-64 & 15.42 & 12.05 & 15.38 & 21.78 & 23.3 & 23.3 & 23.3 & 23.3 & 23.4 & 23.4 & 23.4 & 23.4 & 23.4 & 23.4 \\
\hline ESF-HD-144-TEMP12-RTD-65 & 15.63 & 12.05 & 15.59 & 22.08 & 23.1 & 23.2 & 23.3 & 23.3 & 23.3 & 23.3 & 23.3 & 23.3 & 23.3 & 23.3 \\
\hline ESF-HD-144-TEMP12-RTD-66 & 15.84 & 12.06 & 15.80 & 22.37 & 23.3 & 23.3 & 23.3 & 23.3 & 23.3 & 23.3 & 23.3 & 23.3 & 23.3 & 23.3 \\
\hline ESF-HD-144-TEMP12-RTD-67 & 16.06 & 12.06 & 16.01 & 22.68 & 23.3 & 23.3 & 23.3 & 23.3 & 23.3 & 23.3 & 23.3 & 23.3 & 23.3 & 23.3 \\
\hline \multicolumn{15}{|l|}{ BH 158 Gage ID } \\
\hline ESF-HD-158-TEMP13-RTD-1 & 0.76 & 22.86 & 2.31 & 2.31 & 30.1 & 61.6 & 71.6 & 78.5 & 85.0 & 89.7 & 94.1 & 98.1 & 102.3 & 104.2 \\
\hline ESF-HD-158-TEMP13-RTD-2 & 0.76 & 22.86 & 2.61 & 2.61 & 30.0 & 54.8 & 65.1 & 72.1 & 78.4 & 83.3 & 87.8 & 91.9 & 95.7 & 97.9 \\
\hline ESF-HD-158-TEMP13-RTD-3 & 0.75 & 22.87 & 2.91 & 2.91 & 30.0 & 46.0 & 56.2 & 63.1 & 69.2 & 74.0 & 78.4 & 82.5 & 86.0 & 88.5 \\
\hline ESF-HD-158-TEMP13-RTD-4 & 0.75 & 22.88 & 3.21 & 3.21 & 29.9 & 40.2 & 49.6 & 56.1 & 62.1 & 66.8 & 71.1 & 75.3 & 78.7 & 81.2 \\
\hline ESF-HD-158-TEMP13-RTD-5 & 0.74 & 22.89 & 3.51 & 3.51 & 29.7 & 36.2 & 44.7 & 50.9 & 56.5 & 61.0 & 65.0 & 69.1 & 72.4 & 74.8 \\
\hline ESF-HD-158-TEMP13-RTD-6 & 0.74 & 22.90 & 3.81 & 3.81 & 29.1 & 31.2 & 37.0 & 42.0 & 46.8 & 50.8 & 54.5 & 58.2 & 61.4 & 63.8 \\
\hline ESF-HD-158-TEMP13-RTD-7 & 0.73 & 22.91 & 4.11 & 4.11 & 28.9 & 30.3 & 35.9 & 40.3 & 45.6 & 48.6 & 52.5 & 55.6 & 60.0 & 61.6 \\
\hline ESF-HD-158-TEMP13-RTD-8 & 0.73 & 22.92 & 4.41 & 4.41 & 28.9 & 29.9 & 34.4 & 38.8 & 43.1 & 46.8 & 50.2 & 53.8 & 56.9 & 59.2 \\
\hline
\end{tabular}


First Quarter Report of Temperature Measurements During the Heating Phase of the Drift-Scale Test

\begin{tabular}{|c|c|c|c|c|c|c|c|c|c|c|c|c|c|c|}
\hline \multirow{5}{*}{\begin{tabular}{|l} 
ESF-HD-158-TEMP13-RTD-9 \\
\end{tabular}} & \multicolumn{14}{|c|}{ Temperature Data Obtained from the DST } \\
\hline & \multicolumn{14}{|c|}{ Days after startup } \\
\hline & \multicolumn{4}{|c|}{ As Built Locations (m) } & 0 & 7 & 17 & 27 & 38 & 48 & 58 & 69 & 79 & 87 \\
\hline & $\mathbf{x}$ & $\mathbf{y}$ & $\mathbf{z}$ & D & \multicolumn{10}{|c|}{$\left({ }^{\circ} \mathbf{C}\right)$} \\
\hline & 0.72 & 22.93 & 4.71 & 4.71 & 28.6 & 29.0 & 32.2 & 35.9 & 39.7 & 43.0 & 46.2 & 49.6 & 52.5 & 54.8 \\
\hline ESF-HD-158-TEMP13-RTD-10 & 0.72 & 22.93 & 5.01 & 5.01 & 28.3 & 28.4 & 30.6 & 33.7 & 37.0 & 40.0 & 42.9 & 46.0 & 48.8 & 50.9 \\
\hline ESF-HD-158-TEMP13-RTD-11 & 0.71 & 22.94 & 5.31 & 5.31 & 27.9 & 27.9 & 29.4 & 31.8 & 34.7 & 37.3 & 40.0 & 42.8 & 45.3 & 47.3 \\
\hline ESF-HD-158-TEMP13-RTD-12 & 0.70 & 22.95 & 5.61 & 5.61 & 27.6 & 27.6 & 28.6 & 30.5 & 32.9 & 35.2 & 37.6 & 40.2 & 42.5 & 44.4 \\
\hline ESF-HD-158-TEMP13-RTD-13 & 0.70 & 22.96 & 5.91 & 5.91 & 27.2 & 27.1 & 27.9 & 29.3 & 31.4 & 33.4 & 35.4 & 37.8 & 39.9 & 41.6 \\
\hline ESF-HD-158-TEMP13-RTD-14 & 0.69 & 22.97 & 6.21 & 6.21 & 27.0 & 27.0 & 27.3 & 28.4 & 30.1 & 31.8 & 33.7 & 35.8 & 37.7 & 39.3 \\
\hline ESF-HD-158-TEMP13-RTD-15 & 0.69 & 22.98 & 6.50 & 6.50 & 26.7 & 26.7 & 26.9 & 27.8 & 29.1 & 30.6 & 32.2 & 34.0 & 35.8 & 37.2 \\
\hline ESF-HD-158-TEMP13-RTD-16 & 0.68 & 22.99 & 6.80 & 6.80 & 26.4 & 26.4 & 26.6 & 27.2 & 28.3 & 29.5 & 30.9 & 32.5 & 34.1 & 35.4 \\
\hline ESF-HD-158-TEMP13-RTD-17 & 0.68 & 22.99 & 7.10 & 7.10 & 26.1 & 26.2 & 26.3 & 26.7 & 27.6 & 28.6 & 29.8 & 31.3 & 32.7 & 33.8 \\
\hline ESF-HD-158-TEMP13-RTD-18 & 0.67 & 23.00 & 7.40 & 7.40 & 25.7 & 25.7 & 26.0 & 26.3 & 27.0 & 27.9 & 28.9 & 30.1 & 31.4 & 32.4 \\
\hline ESF-HD-158-TEMP13-RTD-19 & 0.67 & 23.01 & 7.70 & 7.70 & 25.7 & 25.7 & 25.8 & 26.0 & 26.5 & 27.2 & 28.1 & 29.1 & 30.2 & 31.2 \\
\hline ESF-HD-158-TEMP13-RTD-20 & 0.66 & 23.02 & 8.00 & 8.00 & 25.4 & 25.5 & 25.5 & 25.7 & 26.1 & 26.7 & 27.4 & 28.3 & 29.3 & 30.1 \\
\hline ESF-HD-158-TEMP13-RTD-21 & 0.66 & 23.03 & 8.30 & 8.30 & 25.1 & 25.2 & 25.3 & 25.5 & 25.7 & 25.9 & 26.6 & 27.6 & 28.3 & 29.2 \\
\hline ESF-HD-158-TEMP13-RTD-22 & 0.65 & 23.04 & 8.60 & 8.60 & 24.9 & 25.0 & 25.1 & 25.3 & 25.5 & 25.9 & 26.3 & 27.0 & 27.7 & 28.4 \\
\hline ESF-HD-158-TEMP13-RTD-23 & 0.65 & 23.05 & 8.90 & 8.90 & 24.9 & 24.9 & 25.0 & 25.1 & 25.3 & 25.6 & 26.0 & 26.5 & 27.2 & 27.7 \\
\hline ESF-HD-158-TEMP13-RTD-24 & 0.64 & 23.06 & 9.20 & 9.20 & 27.2 & 27.4 & 27.6 & 27.7 & 27.9 & 28.2 & 28.6 & 29.1 & 29.6 & 30.1 \\
\hline ESF-HD-158-TEMP13-RTD-25 & 0.64 & 23.06 & 9.50 & 9.50 & 24.6 & 24.6 & 24.7 & 24.8 & 24.9 & 25.1 & 25.3 & 25.7 & 26.1 & 26.6 \\
\hline ESF-HD-158-TEMP13-RTD-26 & 0.63 & 23.07 & 9.80 & 9.80 & 24.4 & 24.4 & 24.5 & 24.6 & 24.7 & 24.9 & 25.1 & 25.4 & 25.8 & 26.1 \\
\hline ESF-HD-158-TEMP13-RTD-27 & 0.63 & 23.08 & 10.10 & 10.10 & 24.3 & 24.4 & 24.4 & 24.5 & 24.6 & 24.7 & 24.9 & 25.2 & 25.5 & 25.8 \\
\hline ESF-HD-158-TEMP13-RTD-28 & 0.62 & 23.09 & 10.40 & 10.40 & 24.1 & 24.1 & 24.3 & 24.4 & 24.4 & 24.5 & 24.7 & 24.9 & 25.1 & 25.4 \\
\hline ESF-HD-158-TEMP13-RTD-29 & 0.62 & 23.10 & 10.70 & 10.70 & 24.1 & 24.2 & 24.2 & 24.3 & 24.4 & 24.4 & 24.6 & 24.8 & 25.0 & 25.2 \\
\hline ESF-HD-158-TEMP13-RTD-30 & 0.61 & 23.11 & 11.00 & 11.00 & 23.9 & 24.0 & 24.1 & 24.2 & 24.2 & 24.3 & 24.4 & 24.6 & 24.7 & 24.9 \\
\hline ESF-HD-158-TEMP13-RTD-31 & 0.61 & 23.12 & 11.30 & 11.30 & 24.0 & 24.0 & 24.0 & 24.1 & 24.1 & 24.2 & 24.3 & 24.4 & 24.6 & 24.7 \\
\hline ESF-HD-158-TEMP13-RTD-32 & 0.60 & 23.12 & 11.60 & 11.60 & 23.9 & 23.9 & 24.0 & 24.0 & 24.1 & 24.1 & 24.2 & 24.3 & 24.5 & 24.6 \\
\hline ESF-HD-158-TEMP13-RTD-33 & 0.60 & 23.13 & 11.90 & 11.90 & 23.8 & 23.9 & 23.9 & 24.0 & 24.0 & 24.1 & 24.1 & 24.2 & 24.3 & 24.4 \\
\hline ESF-HD-158-TEMP13-RTD-34 & 0.59 & 23.14 & 12.20 & 12.20 & 23.8 & 23.8 & 23.9 & 23.9 & 23.9 & 24.0 & 24.0 & 24.1 & 24.2 & 24.3 \\
\hline ESF-HD-158-TEMP13-RTD-35 & 0.59 & 23.15 & 12.50 & 12.50 & 23.6 & 23.7 & 23.8 & 23.8 & 23.8 & 23.9 & 23.9 & 24.0 & 24.1 & 24.1 \\
\hline ESF-HD-158-TEMP13-RTD-36 & 0.58 & 23.16 & 12.79 & 12.79 & 23.7 & 23.7 & 23.7 & 23.8 & 23.8 & 23.8 & 23.9 & 23.9 & 24.0 & 24.1 \\
\hline ESF-HD-158-TEMP13-RTD-37 & 0.58 & 23.17 & 13.09 & 13.09 & 23.7 & 23.7 & 23.7 & 23.7 & 23.8 & 23.8 & 23.8 & 23.9 & 23.9 & 24.0 \\
\hline ESF-HD-158-TEMP13-RTD-38 & 0.57 & 23.18 & 13.39 & 13.39 & 23.6 & 23.7 & 23.7 & 23.7 & 23.7 & 23.8 & 23.8 & 23.9 & 23.9 & 23.9 \\
\hline ESF-HD-158-TEMP13-RTD-39 & 0.57 & 23.19 & 13.69 & 13.69 & 23.4 & 23.3 & 23.6 & 23.6 & 23.6 & 23.7 & 23.7 & 23.7 & 23.8 & 23.8 \\
\hline ESF-HD-158-TEMP13-RTD-40 & 0.56 & 23.19 & 13.99 & 13.99 & 23.5 & 23.4 & 23.5 & 23.6 & 23.6 & 23.6 & 23.7 & 23.7 & 23.7 & 23.8 \\
\hline ESF-HD-158-TEMP13-RTD-41 & 0.56 & 23.20 & 14.29 & 14.29 & 23.6 & 23.6 & 23.6 & 23.6 & 23.6 & 23.7 & 23.7 & 23.7 & 23.7 & 23.8 \\
\hline ESF-HD-158-TEMP13-RTD-42 & 0.55 & 23.21 & 14.59 & 14.59 & 23.5 & 23.5 & 23.5 & 23.5 & 23.6 & 23.6 & 23.6 & 23.6 & 23.7 & 23.7 \\
\hline ESF-HD-158-TEMP13-RTD-43 & 0.54 & 23.22 & 14.89 & 14.89 & 23.3 & 23.3 & 23.5 & 23.5 & 23.5 & 23.5 & 23.6 & 23.6 & 23.6 & 23.6 \\
\hline ESF-HD-158-TEMP13-RTD-44 & 0.54 & 23.23 & 15.19 & 15.19 & 23.4 & 23.4 & 23.5 & 23.5 & 23.5 & 23.5 & 23.5 & 23.5 & 23.5 & 23.6 \\
\hline ESF-HD-158-TEMP13-RTD-45 & 0.53 & 23.24 & 15.49 & 15.49 & 23.4 & 23.4 & 23.5 & 23.5 & 23.5 & 23.5 & 23.5 & 23.6 & 23.6 & 23.6 \\
\hline
\end{tabular}




\begin{tabular}{|c|c|c|c|c|c|c|c|c|c|c|c|c|c|c|}
\hline & \multicolumn{14}{|c|}{ Temperature Data Obtained from the DST } \\
\hline & \multicolumn{14}{|c|}{ Days after startup } \\
\hline & \multicolumn{4}{|c|}{ As Built Locations (m) } & 0 & 7 & 17 & 27 & 38 & 48 & 58 & 69 & 79 & 87 \\
\hline & $\mathbf{x}$ & $\mathbf{y}$ & $\mathbf{z}$ & D & \multicolumn{10}{|c|}{$\left({ }^{\circ} \mathbf{C}\right)$} \\
\hline ESF-HD-158-TEMP13-RTD-46 & 0.53 & 23.25 & 15.79 & 15.79 & 23.4 & 23.4 & 23.4 & 23.4 & 23.5 & 23.5 & 23.5 & 23.5 & 23.5 & 23.5 \\
\hline ESF-HD-158-TEMP13-RTD-47 & 0.52 & 23.25 & 16.09 & 16.09 & 23.4 & 23.4 & 23.4 & 23.4 & 23.4 & 23.5 & 23.5 & 23.5 & 23.5 & 23.5 \\
\hline ESF-HD-158-TEMP13-RTD-48 & 0.52 & 23.26 & 16.39 & 16.39 & 23.2 & 23.3 & 23.4 & 23.4 & 23.4 & 23.4 & 23.4 & 23.5 & 23.5 & 23.5 \\
\hline ESF-HD-158-TEMP13-RTD-49 & 0.51 & 23.27 & 16.69 & 16.69 & 23.1 & 23.1 & 23.3 & 23.3 & 23.2 & 23.2 & 23.2 & 23.3 & 22.8 & 23.2 \\
\hline ESF-HD-158-TEMP13-RTD-50 & 0.51 & 23.28 & 16.99 & 16.99 & 23.2 & 23.3 & 23.4 & 23.4 & 23.4 & 23.4 & 23.4 & 23.4 & 23.4 & 23.4 \\
\hline ESF-HD-158-TEMP13-RTD-51 & 0.50 & 23.29 & 17.29 & 17.29 & 23.2 & 22.3 & 22.8 & 23.1 & 21.6 & 21.5 & 21.5 & 23.4 & 22.5 & 23.4 \\
\hline ESF-HD-158-TEMP13-RTD-52 & 0.50 & 23.30 & 17.59 & 17.59 & 23.3 & 23.3 & 23.3 & 23.3 & 23.3 & 23.4 & 23.4 & 23.4 & 23.4 & 23.4 \\
\hline ESF-HD-158-TEMP13-RTD-53 & 0.49 & 23.31 & 17.89 & 17.89 & 23.2 & 23.3 & 23.3 & 23.3 & 23.3 & 23.3 & 23.3 & 23.4 & 23.4 & 23.4 \\
\hline ESF-HD-158-TEMP13-RTD-54 & 0.49 & 23.32 & 18.19 & 18.19 & 23.2 & 23.3 & 23.3 & 23.3 & 23.3 & 23.3 & 23.3 & 23.3 & 23.3 & 23.3 \\
\hline ESF-HD-158-TEMP13-RTD-55 & 0.48 & 23.32 & 18.49 & 18.49 & 23.3 & 23.3 & 23.3 & 23.3 & 23.3 & 23.3 & 23.3 & 23.3 & 23.3 & 23.3 \\
\hline ESF-HD-158-TEMP13-RTD-56 & 0.48 & 23.33 & 18.79 & 18.79 & 23.2 & 23.2 & 23.3 & 23.3 & 23.3 & 23.3 & 23.3 & 23.3 & 23.3 & 23.3 \\
\hline ESF-HD-158-TEMP13-RTD-57 & 0.47 & 23.34 & 19.08 & 19.08 & 22.9 & 23.1 & 23.2 & 23.2 & 23.2 & 23.2 & 23.2 & 23.3 & 23.3 & 23.3 \\
\hline ESF-HD-158-TEMP13-RTD-58 & 0.47 & 23.35 & 19.38 & 19.38 & 23.2 & 23.3 & 23.3 & 23.3 & 23.3 & 23.3 & 23.3 & 23.3 & 23.3 & 23.3 \\
\hline ESF-HD-158-TEMP13-RTD-59 & 0.46 & 23.36 & 19.68 & 19.68 & 23.1 & 23.2 & 23.2 & 23.2 & 23.2 & 23.2 & 23.2 & 23.3 & 23.2 & 23.3 \\
\hline ESF-HD-158-TEMP13-RTD-60 & 0.46 & 23.37 & 19.98 & 19.98 & 23.2 & 23.2 & 23.3 & 23.3 & 23.3 & 23.3 & 23.3 & 23.3 & 23.3 & 23.3 \\
\hline ESF-HD-158-TEMP13-RTD-61 & 0.45 & 23.38 & 20.28 & 20.28 & 23.1 & 23.2 & 23.3 & 23.3 & 23.3 & 23.3 & 23.3 & 23.3 & 23.3 & 23.3 \\
\hline ESF-HD-158-TEMP13-RTD-62 & 0.45 & 23.38 & 20.58 & 20.58 & 23.1 & 23.2 & 23.2 & 23.2 & 23.2 & 23.2 & 23.2 & 23.2 & 23.2 & 23.2 \\
\hline ESF-HD-158-TEMP13-RTD-63 & 0.44 & 23.39 & 20.88 & 20.88 & 23.2 & 23.2 & 23.2 & 23.2 & 23.2 & 23.2 & 23.2 & 23.2 & 23.2 & 23.2 \\
\hline ESF-HD-158-TEMP13-RTD-64 & 0.44 & 23.40 & 21.18 & 21.18 & 23.1 & 23.0 & 23.2 & 23.2 & 22.9 & 23.1 & 23.2 & 23.2 & 23.2 & 23.2 \\
\hline ESF-HD-158-TEMP13-RTD-65 & 0.43 & 23.41 & 21.48 & 21.48 & 23.2 & 23.2 & 23.2 & 23.2 & 23.2 & 23.2 & 23.2 & 23.3 & 23.3 & 23.3 \\
\hline ESF-HD-158-TEMP13-RTD-66 & 0.43 & 23.42 & 21.78 & 21.78 & 23.2 & 23.2 & 23.2 & 23.2 & 23.2 & 23.2 & 23.2 & 23.2 & 23.2 & 23.2 \\
\hline ESF-HD-158-TEMP13-RTD-67 & 0.42 & 23.43 & 22.08 & 22.08 & 23.1 & 23.2 & 23.2 & 23.2 & 23.2 & 23.2 & 23.2 & 23.2 & 23.2 & 23.2 \\
\hline \multicolumn{15}{|l|}{ BH 159 Gage ID } \\
\hline ESF-HD-159-TEMP14-RTD-1 & -1.71 & 22.88 & 1.69 & 2.40 & 30.0 & 60.7 & 70.7 & 77.7 & 84.2 & 89.1 & 93.6 & 97.6 & 101.6 & 103.8 \\
\hline ESF-HD-159-TEMP14-RTD-2 & -1.93 & 22.88 & 1.91 & 2.72 & 30.0 & 59.1 & 69.1 & 76.0 & 82.4 & 87.4 & 91.9 & 96.0 & 100.0 & 102.3 \\
\hline ESF-HD-159-TEMP14-RTD-3 & -2.14 & 22.88 & 2.13 & 3.02 & 29.9 & 47.0 & 57.3 & 64.4 & 70.9 & 76.0 & 80.6 & 84.9 & 88.6 & 91.1 \\
\hline ESF-HD-159-TEMP14-RTD-4 & -2.36 & 22.88 & 2.34 & 3.32 & 30.0 & 40.5 & 50.0 & 56.8 & 63.1 & 68.1 & 72.6 & 77.0 & 80.7 & 83.4 \\
\hline ESF-HD-159-TEMP14-RTD-5 & -2.57 & 22.88 & 2.56 & 3.63 & 29.7 & 36.2 & 44.7 & 51.1 & 57.1 & 62.0 & 66.4 & 70.8 & 74.5 & 77.2 \\
\hline ESF-HD-159-TEMP14-RTD-6 & -2.79 & 22.88 & 2.78 & 3.94 & 29.4 & 33.1 & 40.3 & 46.2 & 51.9 & 56.7 & 60.9 & 65.3 & 68.9 & 71.7 \\
\hline ESF-HD-159-TEMP14-RTD-7 & -3.00 & 22.88 & 2.99 & 4.24 & 29.2 & 31.2 & 37.0 & 42.3 & 47.7 & 52.3 & 56.4 & 60.7 & 64.3 & 67.0 \\
\hline ESF-HD-159-TEMP14-RTD-8 & -3.21 & 22.88 & 3.21 & 4.54 & 28.8 & 29.8 & 34.3 & 39.0 & 43.9 & 48.2 & 52.2 & 56.4 & 59.9 & 62.6 \\
\hline ESF-HD-159-TEMP14-RTD-9 & -3.43 & 22.88 & 3.43 & 4.85 & 28.5 & 28.8 & 32.2 & 36.3 & 40.8 & 44.8 & 48.7 & 52.8 & 56.2 & 58.9 \\
\hline ESF-HD-159-TEMP14-RTD-10 & -3.64 & 22.88 & 3.65 & 5.15 & 28.2 & 28.2 & 30.6 & 34.0 & 38.0 & 41.7 & 45.3 & 49.2 & 52.5 & 55.1 \\
\hline ESF-HD-159-TEMP14-RTD-11 & -3.86 & 22.88 & 3.86 & 5.46 & 27.8 & 27.8 & 29.5 & 32.2 & 35.8 & 39.2 & 42.6 & 46.3 & 49.5 & 52.0 \\
\hline ESF-HD-159-TEMP14-RTD-12 & -4.07 & 22.89 & 4.08 & 5.76 & 27.4 & 27.4 & 28.5 & 30.7 & 33.7 & 36.8 & 39.9 & 43.4 & 46.4 & 48.9 \\
\hline ESF-HD-159-TEMP14-RTD-13 & -4.29 & 22.89 & 4.30 & 6.07 & 27.1 & 27.1 & 27.9 & 29.6 & 32.3 & 35.0 & 38.0 & 41.2 & 44.1 & 46.5 \\
\hline ESF-HD-159-TEMP14-RTD-14 & -4.50 & 22.89 & 4.51 & 6.37 & 26.9 & 26.9 & 27.3 & 28.7 & 30.9 & 33.4 & 36.0 & 39.0 & 41.7 & 43.9 \\
\hline
\end{tabular}


First Quarter Report of Temperature Measurements During the Heating Phase of the Drift-Scale Test

\begin{tabular}{|c|c|c|c|c|c|c|c|c|c|c|c|c|c|c|}
\hline \multirow{5}{*}{\begin{tabular}{|l|} 
\\
ESF-HD-159-TEMP14-RTD-15 \\
\end{tabular}} & \multicolumn{14}{|c|}{ Temperature Data Obtained from the DST } \\
\hline & \multicolumn{14}{|c|}{ Days after startup } \\
\hline & \multicolumn{4}{|c|}{ As Built Locations (m) } & 0 & 7 & 17 & 27 & 38 & 48 & 58 & 69 & 79 & 87 \\
\hline & \multirow{2}{*}{\begin{tabular}{c|}
$\mathbf{x}$ \\
-4.72
\end{tabular}} & \multirow{2}{*}{$\begin{array}{c}\mathbf{y} \\
22.89\end{array}$} & \multirow{2}{*}{\begin{tabular}{c|}
$\mathbf{z}$ \\
4.73
\end{tabular}} & \multirow{2}{*}{\begin{tabular}{l|}
$\mathbf{D}$ \\
6.68 \\
\end{tabular}} & \multicolumn{10}{|c|}{$\left({ }^{\circ} \mathrm{C}\right)$} \\
\hline & & & & & 26.6 & 26.6 & 26.9 & 28.0 & 29.9 & 32.0 & 34.4 & 37.2 & 39.7 & 41.8 \\
\hline ESF-HD-159-TEMP14-RTD-16 & -4.93 & 22.89 & 4.95 & 6.99 & 26.2 & 26.4 & 26.7 & 27.4 & 29.0 & 30.8 & 32.9 & 35.4 & 37.8 & 39.8 \\
\hline ESF-HD-159-TEMP14-RTD-17 & -5.15 & 22.89 & 5.17 & 7.30 & 26.0 & 26.1 & 26.2 & 26.8 & 28.1 & 29.7 & 31.6 & 33.9 & 36.1 & 37.9 \\
\hline ESF-HD-159-TEMP14-RTD-18 & -5.36 & 22.89 & 5.38 & 7.59 & 25.8 & 25.9 & 26.0 & 26.4 & 27.4 & 28.8 & 30.5 & 32.5 & 34.6 & 36.3 \\
\hline ESF-HD-159-TEMP14-RTD-19 & -5.57 & 22.89 & 5.60 & 7.90 & 25.5 & 25.6 & 25.7 & 26.1 & 26.9 & 28.0 & 29.5 & 31.4 & 33.3 & 34.8 \\
\hline ESF-HD-159-TEMP14-RTD-20 & -5.79 & 22.89 & 5.82 & 8.21 & 25.3 & 25.4 & 25.5 & 25.8 & 26.4 & 27.4 & 28.7 & 30.4 & 32.0 & 33.5 \\
\hline ESF-HD-159-TEMP14-RTD-21 & -6.00 & 22.89 & 6.03 & 8.51 & 25.1 & 25.2 & 25.3 & 25.5 & 26.1 & 26.9 & 28.0 & 29.5 & 31.0 & 32.4 \\
\hline ESF-HD-159-TEMP14-RTD-22 & -6.22 & 22.89 & 6.25 & 8.82 & 25.0 & 25.1 & 25.2 & 25.3 & 25.8 & 26.5 & 27.4 & 28.7 & 30.1 & 31.3 \\
\hline ESF-HD-159-TEMP14-RTD-23 & -6.43 & 22.90 & 6.47 & 9.12 & 24.9 & 25.0 & 25.0 & 25.2 & 25.5 & 26.0 & 26.8 & 28.1 & 29.3 & 30.4 \\
\hline ESF-HD-159-TEMP14-RTD-24 & -6.65 & 22.90 & 6.68 & 9.43 & 24.7 & 24.8 & 24.9 & 25.0 & 25.2 & 25.7 & 26.4 & 27.4 & 28.5 & 29.5 \\
\hline ESF-HD-159-TEMP14-RTD-25 & -6.86 & 22.90 & 6.90 & 9.73 & 24.6 & 24.7 & 24.7 & 24.8 & 25.0 & 25.4 & 26.0 & 26.9 & 27.9 & 28.8 \\
\hline ESF-HD-159-TEMP14-RTD-26 & -7.08 & 22.90 & 7.12 & 10.04 & 24.5 & 24.5 & 24.6 & 24.7 & 24.8 & 25.2 & & 26.4 & 27.3 & 28.1 \\
\hline ESF-HD-159-TEMP14-RTD-27 & -7.29 & 22.90 & 7.34 & 10.35 & 24.3 & 24.2 & 24.3 & 24.4 & 24.5 & 24.8 & 25.2 & 25.9 & 26.6 & 27.3 \\
\hline ESF-HD-159-TEMP14-RTD-28 & -7.51 & 22.90 & 7.55 & 10.65 & 24.1 & 24.3 & 24.4 & 24.4 & 24.6 & 24.8 & 25.1 & 25.7 & 26.4 & 27.0 \\
\hline ESF-HD-159-TEMP14-RTD-29 & -7.72 & 22.90 & 7.77 & 10.95 & 24.1 & 24.1 & 24.3 & 24.3 & 24.4 & 24.6 & 24.9 & 25.4 & 25.9 & 26.5 \\
\hline ESF-HD-159-TEMP14-RTD-30 & -7.93 & 22.90 & 7.99 & 11.26 & 23.9 & 24.1 & 24.2 & 24.2 & 24.3 & 24.5 & 24.7 & 25.1 & 25.6 & 26.1 \\
\hline ESF-HD-159-TEMP14-RTD-31 & -8.15 & 22.90 & 8.20 & 11.56 & 24.0 & 24.0 & 24.1 & 24.2 & 24.2 & 24.3 & 24.6 & 24.9 & 25.4 & 25.8 \\
\hline ESF-HD-159-TEMP14-RTD-32 & -8.36 & 22.90 & 8.42 & 11.87 & 24.0 & 24.0 & 24.1 & 24.1 & 24.2 & 24.3 & 24.5 & 24.8 & 25.1 & 25.5 \\
\hline ESF-HD-159-TEMP14-RTD-33 & -8.58 & 22.90 & 8.64 & 12.18 & 23.9 & 23.9 & 24.0 & 24.0 & 24.1 & 24.2 & 24.3 & 24.6 & 24.9 & 25.3 \\
\hline ESF-HD-159-TEMP14-RTD-34 & -8.79 & 22.90 & 8.86 & 12.48 & 23.7 & 23.7 & 23.9 & 23.9 & 24.0 & 24.0 & 24.2 & 24.4 & 24.7 & 24.9 \\
\hline ESF-HD-159-TEMP14-RTD-35 & -9.01 & 22.91 & 9.07 & 12.79 & 23.8 & 23.8 & 23.9 & 23.9 & 23.9 & 24.0 & 24.1 & 24.3 & 24.5 & 24.8 \\
\hline ESF-HD-159-TEMP14-RTD-36 & -9.22 & 22.91 & 9.29 & 13.09 & 23.7 & 23.6 & 23.8 & 23.9 & 23.9 & 23.9 & 24.0 & 24.2 & 24.4 & 24.6 \\
\hline ESF-HD-159-TEMP14-RTD-37 & -9.44 & 22.91 & 9.51 & 13.40 & 23.7 & 23.7 & 23.8 & 23.8 & 23.8 & 23.9 & 23.9 & 24.1 & 24.2 & 24.4 \\
\hline ESF-HD-159-TEMP14-RTD-38 & -9.65 & 22.91 & 9.72 & 13.70 & 23.7 & 23.7 & 23.7 & 23.8 & 23.8 & 23.8 & 23.9 & 24.0 & 24.1 & 24.3 \\
\hline ESF-HD-159-TEMP14-RTD-39 & -9.86 & 22.91 & 9.94 & 14.00 & 23.7 & 23.6 & 23.7 & 23.7 & 23.8 & 23.8 & 23.8 & 23.9 & 24.1 & 24.2 \\
\hline ESF-HD-159-TEMP14-RTD-40 & -10.08 & 22.91 & 10.16 & 14.31 & 23.5 & 23.5 & 23.6 & 23.7 & 23.7 & 23.7 & 23.8 & 23.8 & 23.9 & 24.1 \\
\hline ESF-HD-159-TEMP14-RTD-41 & -10.29 & 22.91 & 10.38 & 14.62 & 23.6 & 23.0 & 23.5 & 23.6 & 23.5 & 23.3 & 23.6 & 23.8 & 23.7 & 23.9 \\
\hline ESF-HD-159-TEMP14-RTD-42 & -10.51 & 22.91 & 10.59 & 14.92 & 23.4 & 23.3 & 23.5 & 23.6 & 23.6 & 23.6 & 23.6 & 23.7 & 23.8 & 23.8 \\
\hline ESF-HD-159-TEMP14-RTD-43 & -10.72 & 22.91 & 10.81 & 15.22 & 23.5 & 23.3 & 23.5 & 23.6 & 23.6 & 23.6 & 23.6 & 23.7 & 23.7 & 23.8 \\
\hline ESF-HD-159-TEMP14-RTD-44 & -10.94 & 22.91 & 11.03 & 15.54 & 23.4 & 23.4 & 23.5 & 23.5 & 23.5 & 23.6 & 23.6 & 23.6 & 23.6 & 23.7 \\
\hline ESF-HD-159-TEMP14-RTD-45 & -11.15 & 22.91 & 11.24 & 15.83 & 23.4 & 23.4 & 23.5 & 23.5 & 23.5 & 23.6 & 23.6 & 23.6 & 23.7 & 23.7 \\
\hline ESF-HD-159-TEMP14-RTD-46 & -11.37 & 22.91 & 11.46 & 16.14 & 23.5 & 23.5 & 23.5 & 23.5 & 23.5 & 23.5 & 23.6 & 23.6 & 23.6 & 23.7 \\
\hline ESF-HD-159-TEMP14-RTD-47 & -11.58 & 22.92 & 11.68 & 16.45 & 23.5 & 23.5 & 23.5 & 23.5 & 23.5 & 23.5 & 23.5 & 23.6 & 23.6 & 23.6 \\
\hline ESF-HD-159-TEMP14-RTD-48 & -11.80 & 22.92 & 11.90 & 16.76 & 23.3 & 23.3 & 23.5 & 23.5 & 23.5 & 23.5 & 23.5 & 23.5 & 23.6 & 23.6 \\
\hline ESF-HD-159-TEMP14-RTD-49 & -12.01 & 22.92 & 12.11 & 17.06 & 23.4 & 23.4 & 23.5 & 23.5 & 23.5 & 23.5 & 23.5 & 23.5 & 23.5 & 23.6 \\
\hline ESF-HD-159-TEMP14-RTD-50 & -12.22 & 22.92 & 12.33 & 17.36 & 23.3 & 23.3 & 23.4 & 23.5 & 23.5 & 23.5 & 23.5 & 23.5 & 23.5 & 23.5 \\
\hline ESF-HD-159-TEMP14-RTD-51 & -12.44 & 22.92 & 12.55 & 17.67 & 23.4 & 23.4 & 23.4 & 23.4 & 23.4 & 23.4 & 23.5 & 23.5 & 23.5 & 23.5 \\
\hline
\end{tabular}




\begin{tabular}{|c|c|c|c|c|c|c|c|c|c|c|c|c|c|c|}
\hline & \multicolumn{14}{|c|}{ Temperature Data Obtained from the DST } \\
\hline & \multicolumn{14}{|c|}{ Days after startup } \\
\hline & \multicolumn{4}{|c|}{ As Built Locations (m) } & 0 & 7 & 17 & 27 & 38 & 48 & 58 & 69 & 79 & 87 \\
\hline & $\mathbf{x}$ & $\mathbf{y}$ & $\mathbf{z}$ & D & \multicolumn{10}{|c|}{$\left({ }^{\circ} \mathbf{C}\right)$} \\
\hline ESF-HD-159-TEMP14-RTD-52 & -12.65 & 22.92 & 12.76 & 17.97 & 23.4 & 23.4 & 23.4 & 23.4 & 23.4 & 23.4 & 23.4 & 23.4 & 23.4 & 23.5 \\
\hline ESF-HD-159-TEMP14-RTD-53 & -12.87 & 22.92 & 12.98 & 18.28 & 23.2 & 23.4 & 23.5 & 23.5 & 23.5 & 23.5 & 23.5 & 23.5 & 23.5 & 23.6 \\
\hline ESF-HD-159-TEMP14-RTD-54 & -13.08 & 22.92 & 13.20 & 18.58 & 23.4 & 23.3 & 23.4 & 23.4 & 23.4 & 23.4 & 23.4 & 23.4 & 23.5 & 23.5 \\
\hline ESF-HD-159-TEMP14-RTD-55 & -13.30 & 22.92 & 13.41 & 18.89 & 23.4 & 23.4 & 23.4 & 23.4 & 23.4 & 23.4 & 23.4 & 23.4 & 23.4 & 23.4 \\
\hline ESF-HD-159-TEMP14-RTD-56 & -13.51 & 22.92 & 13.63 & 19.19 & 23.4 & 23.4 & 23.4 & 23.4 & 23.4 & 23.4 & 23.4 & 23.4 & 23.4 & 23.4 \\
\hline ESF-HD-159-TEMP14-RTD-57 & -13.73 & 22.92 & 13.85 & 19.50 & 23.4 & 23.4 & 23.4 & 23.4 & 23.4 & 23.4 & 23.4 & 23.4 & 23.4 & 23.4 \\
\hline ESF-HD-159-TEMP14-RTD-58 & -13.94 & 22.92 & 14.07 & 19.81 & 23.4 & 23.4 & 23.4 & 23.4 & 23.4 & 23.4 & 23.4 & 23.4 & 23.4 & 23.4 \\
\hline ESF-HD-159-TEMP14-RTD-59 & -14.15 & 22.93 & 14.28 & 20.10 & 23.4 & 23.4 & 23.4 & 23.4 & 23.4 & 23.4 & 23.4 & 23.4 & 23.4 & 23.4 \\
\hline ESF-HD-159-TEMP14-RTD-60 & -14.37 & 22.93 & 14.50 & 20.41 & 23.4 & 23.4 & 23.4 & 23.4 & 23.4 & 23.4 & 23.4 & 23.4 & 23.4 & 23.4 \\
\hline ESF-HD-159-TEMP14-RTD-61 & -14.58 & 22.93 & 14.72 & 20.72 & 23.3 & 23.3 & 23.3 & 23.3 & 23.3 & 23.3 & 23.3 & 23.3 & 23.3 & 23.3 \\
\hline ESF-HD-159-TEMP14-RTD-62 & -14.80 & 22.93 & 14.93 & 21.02 & 23.1 & 23.2 & 23.3 & 23.3 & 23.3 & 23.3 & 23.3 & 23.3 & 23.3 & 23.3 \\
\hline ESF-HD-159-TEMP14-RTD-63 & -15.01 & 22.93 & 15.15 & 21.33 & 23.4 & 23.4 & 23.3 & 23.4 & 23.4 & 23.4 & 23.4 & 23.4 & 23.4 & 23.4 \\
\hline ESF-HD-159-TEMP14-RTD-64 & -15.23 & 22.93 & 15.37 & 21.64 & 23.3 & 23.3 & 23.3 & 23.3 & 23.3 & 23.3 & 23.3 & 23.3 & 23.3 & 23.4 \\
\hline ESF-HD-159-TEMP14-RTD-65 & -15.44 & 22.93 & 15.59 & 21.94 & 23.3 & 23.3 & 23.3 & 23.3 & 23.3 & 23.3 & 23.3 & 23.3 & 23.3 & 23.3 \\
\hline ESF-HD-159-TEMP14-RTD-66 & -15.66 & 22.93 & 15.80 & 22.25 & 23.2 & 23.3 & 23.3 & 23.3 & 23.4 & 23.4 & 23.4 & 23.4 & 23.4 & 23.4 \\
\hline ESF-HD-159-TEMP14-RTD-67 & -15.87 & 22.93 & 16.02 & 22.55 & 23.3 & 23.3 & 23.3 & 23.3 & 23.3 & 23.3 & 23.3 & 23.3 & 23.3 & 23.4 \\
\hline \multicolumn{15}{|l|}{ BH 160 Gage ID } \\
\hline ESF-HD-160-TEMP15-RTD-1 & -2.22 & 22.87 & -0.01 & 2.22 & 30.1 & 59.0 & & 76.2 & & 87.8 & 92.4 & 96.6 & 100.6 & 102.8 \\
\hline ESF-HD-160-TEMP15-RTD-2 & -2.52 & 22.87 & 0.00 & 2.52 & 29.9 & 54.5 & 69.0 & 73.0 & 82.7 & 85.1 & 89.8 & 94.1 & 97.9 & 100.5 \\
\hline ESF-HD-160-TEMP15-RTD-3 & -2.82 & 22.87 & 0.00 & 2.82 & 30.0 & 46.6 & 52.1 & 66.5 & 70.9 & 79.6 & 84.4 & 89.3 & 91.9 & 96.2 \\
\hline ESF-HD-160-TEMP15-RTD-4 & -3.13 & 22.87 & 0.00 & 3.13 & 29.7 & 41.3 & 53.8 & 62.0 & 72.8 & 75.9 & 81.1 & 85.9 & 90.0 & 93.1 \\
\hline ESF-HD-160-TEMP15-RTD-5 & -3.43 & 22.87 & 0.00 & 3.43 & 29.6 & 37.7 & 55.1 & 59.0 & 74.4 & 73.4 & 78.7 & 83.7 & 87.8 & 91.2 \\
\hline ESF-HD-160-TEMP15-RTD-6 & -3.73 & 22.88 & 0.00 & 3.73 & 29.4 & 36.5 & 56.5 & 57.5 & 76.0 & 72.4 & 77.9 & 82.9 & 87.2 & 90.6 \\
\hline ESF-HD-160-TEMP15-RTD-7 & -4.03 & 22.88 & 0.00 & 4.03 & 29.3 & 36.1 & 56.9 & 57.2 & 76.5 & 72.5 & 78.1 & 83.3 & 87.7 & 91.1 \\
\hline ESF-HD-160-TEMP15-RTD-8 & -4.33 & 22.88 & 0.01 & 4.33 & 28.9 & 36.6 & 57.5 & 58.0 & 77.2 & 73.5 & 79.2 & 84.5 & 88.9 & 92.2 \\
\hline ESF-HD-160-TEMP15-RTD-9 & -4.63 & 22.88 & 0.01 & 4.63 & 28.7 & 38.0 & 57.7 & 59.8 & 77.5 & 75.5 & 81.2 & 86.7 & 91.0 & 94.3 \\
\hline ESF-HD-160-TEMP15-RTD-10 & -4.93 & 22.88 & 0.01 & 4.93 & 28.2 & 39.2 & 58.0 & 61.7 & 77.9 & 77.5 & 83.3 & 88.8 & 93.0 & 96.3 \\
\hline ESF-HD-160-TEMP15-RTD-11 & -5.23 & 22.88 & 0.01 & 5.23 & 28.1 & 40.6 & 57.8 & 63.7 & 77.7 & 79.6 & 85.4 & 90.8 & 94.6 & 96.6 \\
\hline ESF-HD-160-TEMP15-RTD-12 & -5.53 & 22.88 & 0.01 & 5.53 & 27.8 & 41.4 & 57.9 & 65.3 & 77.7 & 81.3 & 86.9 & 92.2 & 95.5 & 96.8 \\
\hline ESF-HD-160-TEMP15-RTD-13 & -5.83 & 22.89 & 0.01 & 5.83 & 27.4 & 42.2 & 65.5 & 66.9 & 79.8 & 83.0 & 88.5 & 93.6 & 96.1 & 96.9 \\
\hline ESF-HD-160-TEMP15-RTD-14 & -6.14 & 22.89 & 0.02 & 6.14 & 26.9 & 42.1 & 57.2 & 67.4 & 76.9 & 83.5 & 88.9 & 94.0 & 96.0 & 96.9 \\
\hline ESF-HD-160-TEMP15-RTD-15 & -6.44 & 22.89 & 0.02 & 6.44 & 26.8 & 42.5 & 56.6 & 68.2 & 76.0 & 84.2 & 89.6 & 94.8 & 96.3 & 97.0 \\
\hline ESF-HD-160-TEMP15-RTD-16 & -6.74 & 22.89 & 0.02 & 6.74 & 26.5 & 42.1 & 54.5 & 68.5 & 74.0 & 84.4 & 89.9 & 95.2 & 96.2 & 96.8 \\
\hline ESF-HD-160-TEMP15-RTD-17 & -7.04 & 22.89 & 0.02 & 7.04 & 26.3 & 42.3 & 55.1 & 68.9 & 74.1 & 84.8 & 90.3 & 95.2 & 96.3 & 96.9 \\
\hline ESF-HD-160-TEMP15-RTD-18 & -7.34 & 22.89 & 0.02 & 7.34 & 25.9 & 41.8 & 54.7 & 68.6 & 73.9 & 84.5 & 90.0 & 94.9 & 96.1 & 96.8 \\
\hline ESF-HD-160-TEMP15-RTD-19 & -7.64 & 22.89 & 0.02 & 7.64 & 25.7 & 41.9 & 55.1 & 68.7 & 74.8 & 84.5 & 90.0 & 95.2 & 96.2 & 96.8 \\
\hline ESF-HD-160-TEMP15-RTD-20 & -7.94 & 22.89 & 0.03 & 7.94 & 25.4 & 41.2 & 55.7 & 67.9 & 76.5 & 83.7 & 89.2 & 94.7 & 96.2 & 96.7 \\
\hline
\end{tabular}


First Quarter Report of Temperature Measurements During the Heating Phase of the Drift-Scale Test

\begin{tabular}{|c|c|c|c|c|c|c|c|c|c|c|c|c|c|c|}
\hline \multirow{5}{*}{\begin{tabular}{|l|} 
\\
ESF-HD-160-TEMP15-RTD-21 \\
\end{tabular}} & \multicolumn{14}{|c|}{ Temperature Data Obtained from the DST } \\
\hline & \multicolumn{14}{|c|}{ Days after startup } \\
\hline & \multicolumn{4}{|c|}{ As Built Locations (m) } & 0 & 7 & 17 & 27 & 38 & 48 & 58 & 69 & 79 & 87 \\
\hline & $\mathbf{x}$ & $\mathbf{y}$ & $\mathbf{z}$ & D & \multicolumn{10}{|c|}{$\left({ }^{\circ} \mathbf{C}\right)$} \\
\hline & -8.24 & 22.90 & 0.03 & 8.24 & 25.4 & 40.6 & 59.4 & 66.9 & 83.1 & 82.9 & 88.6 & 93.8 & 96.3 & 96.8 \\
\hline ESF-HD-160-TEMP15-RTD-22 & -8.54 & 22.90 & 0.03 & 8.54 & 24.5 & 38.8 & 57.9 & 64.6 & 80.8 & 81.6 & 87.6 & 92.9 & 95.6 & 96.6 \\
\hline ESF-HD-160-TEMP15-RTD-23 & -8.85 & 22.90 & 0.03 & 8.85 & 25.1 & 39.2 & 56.8 & 64.9 & 78.9 & 81.2 & 87.3 & 92.7 & 95.4 & 96.5 \\
\hline ESF-HD-160-TEMP15-RTD-24 & -9.15 & 22.90 & 0.03 & 9.15 & 25.0 & 38.9 & 58.5 & 64.5 & 74.1 & 81.2 & 87.5 & 92.6 & 95.3 & 96.3 \\
\hline ESF-HD-160-TEMP15-RTD-25 & -9.45 & 22.90 & 0.03 & 9.45 & 24.8 & 39.1 & 60.3 & 65.1 & 83.9 & 82.4 & 88.5 & 93.2 & 95.5 & 96.3 \\
\hline ESF-HD-160-TEMP15-RTD-26 & -9.75 & 22.90 & 0.04 & 9.75 & 24.7 & 39.6 & 61.1 & 66.4 & 84.2 & 84.0 & 89.6 & 94.0 & 95.7 & 96.3 \\
\hline ESF-HD-160-TEMP15-RTD-27 & -10.05 & 22.90 & 0.04 & 10.05 & 24.1 & 42.3 & 61.5 & 72.7 & 83.8 & 89.2 & 93.7 & 96.5 & 96.3 & 96.8 \\
\hline ESF-HD-160-TEMP15-RTD-28 & -10.35 & 22.90 & 0.04 & 10.35 & 24.5 & 41.3 & 62.4 & 70.2 & 83.8 & 87.3 & 92.2 & 95.7 & 96.3 & 96.8 \\
\hline ESF-HD-160-TEMP15-RTD-29 & -10.65 & 22.91 & 0.04 & 10.65 & 24.5 & 40.5 & 62.1 & 68.4 & 82.4 & 85.9 & 91.1 & 94.9 & 96.1 & 96.6 \\
\hline ESF-HD-160-TEMP15-RTD-30 & -10.95 & 22.91 & 0.04 & 10.95 & 24.4 & 42.8 & 61.6 & 73.6 & 80.8 & 90.0 & 94.3 & 96.3 & 96.5 & 97.0 \\
\hline ESF-HD-160-TEMP15-RTD-31 & -11.25 & 22.91 & 0.04 & 11.25 & 24.2 & 43.2 & 60.8 & 74.4 & 78.8 & 90.5 & 94.6 & 96.4 & 96.5 & 97.5 \\
\hline ESF-HD-160-TEMP15-RTD-32 & -11.56 & 22.91 & 0.05 & 11.56 & 24.2 & 43.6 & 59.2 & 74.5 & 76.1 & 90.5 & 94.6 & 96.4 & 96.4 & 97.1 \\
\hline ESF-HD-160-TEMP15-RTD-33 & -11.86 & 22.91 & 0.05 & 11.86 & 24.2 & 44.4 & 56.4 & 75.1 & 72.3 & 90.4 & 94.4 & 96.4 & 96.4 & 97.1 \\
\hline ESF-HD-160-TEMP15-RTD-34 & -12.16 & 22.91 & 0.05 & 12.16 & 24.1 & 44.5 & 53.0 & 74.4 & 68.0 & 88.8 & 93.1 & 95.8 & 96.4 & 96.8 \\
\hline ESF-HD-160-TEMP15-RTD-35 & -12.46 & 22.91 & 0.05 & 12.46 & 24.0 & 44.7 & 53.8 & 73.7 & 70.2 & 86.9 & 91.3 & 94.6 & 96.2 & 96.7 \\
\hline ESF-HD-160-TEMP15-RTD-36 & -12.76 & 22.91 & 0.05 & 12.76 & 24.0 & 44.9 & 48.5 & 72.7 & 62.1 & 84.7 & 89.1 & 93.1 & 94.9 & 96.2 \\
\hline ESF-HD-160-TEMP15-RTD-37 & -13.06 & 22.92 & 0.05 & 13.06 & 24.0 & 44.3 & 42.9 & 70.8 & 55.2 & 81.9 & 86.0 & 91.2 & 92.4 & 94.5 \\
\hline ESF-HD-160-TEMP15-RTD-38 & -13.36 & 22.92 & 0.06 & 13.36 & 23.9 & 42.3 & 37.5 & 67.4 & 48.7 & 78.0 & 81.8 & 87.8 & 89.1 & 92.3 \\
\hline ESF-HD-160-TEMP15-RTD-39 & -13.66 & 22.92 & 0.06 & 13.66 & 23.7 & 39.9 & 33.4 & 63.3 & 43.0 & 73.5 & 77.1 & 83.4 & 85.1 & 89.2 \\
\hline ESF-HD-160-TEMP15-RTD-40 & -13.96 & 22.92 & 0.06 & 13.96 & 23.8 & 37.0 & 30.2 & 57.7 & 38.4 & 67.4 & 70.9 & 76.1 & 78.6 & 82.6 \\
\hline ESF-HD-160-TEMP15-RTD-41 & -14.27 & 22.92 & 0.06 & 14.27 & 23.7 & 33.0 & 28.1 & 50.8 & 35.0 & 60.2 & 63.6 & 67.9 & 70.9 & 74.5 \\
\hline ESF-HD-160-TEMP15-RTD-42 & -14.57 & 22.92 & 0.06 & 14.57 & 23.8 & 29.4 & 26.6 & 44.2 & 32.3 & 53.2 & 56.6 & 60.0 & 63.0 & 66.0 \\
\hline ESF-HD-160-TEMP15-RTD-43 & -14.87 & 22.92 & 0.06 & 14.87 & 23.7 & 27.0 & 25.6 & 38.8 & 30.3 & 47.0 & 50.3 & 53.3 & 56.1 & 58.6 \\
\hline ESF-HD-160-TEMP15-RTD-44 & -15.17 & 22.92 & 0.07 & 15.17 & 23.6 & 25.4 & 24.9 & 34.6 & 28.7 & 41.8 & 44.8 & 47.6 & 50.3 & 52.5 \\
\hline ESF-HD-160-TEMP15-RTD-45 & -15.47 & 22.93 & 0.07 & 15.47 & 23.8 & 24.6 & 24.4 & 31.6 & 27.5 & 38.1 & 40.9 & 43.5 & 46.0 & 47.8 \\
\hline ESF-HD-160-TEMP15-RTD-46 & -15.77 & 22.93 & 0.07 & 15.77 & 23.6 & 24.1 & 50.7 & 29.3 & 67.7 & 35.0 & 37.5 & 39.9 & 42.2 & 43.9 \\
\hline ESF-HD-160-TEMP15-RTD-47 & -16.07 & 22.93 & 0.07 & 16.07 & 23.7 & 23.9 & 24.1 & 27.8 & 26.5 & 32.6 & 34.9 & 37.1 & 39.2 & 40.7 \\
\hline ESF-HD-160-TEMP15-RTD-48 & -16.37 & 22.93 & 0.07 & 16.37 & 23.7 & 23.8 & 23.9 & 26.6 & 25.7 & 30.7 & 32.7 & 34.7 & 36.6 & 38.0 \\
\hline ESF-HD-160-TEMP15-RTD-49 & -16.67 & 22.93 & 0.07 & 16.67 & 23.7 & 23.6 & 23.8 & 25.7 & 25.2 & 29.1 & 30.9 & 32.7 & 34.4 & 35.7 \\
\hline ESF-HD-160-TEMP15-RTD-50 & -16.98 & 22.93 & 0.08 & 16.98 & 23.7 & 23.7 & 23.7 & 25.1 & 24.8 & 28.0 & 29.5 & 31.1 & 32.6 & 33.8 \\
\hline ESF-HD-160-TEMP15-RTD-51 & -17.28 & 22.93 & 0.08 & 17.28 & 23.6 & 23.6 & 23.7 & 24.6 & 24.5 & 26.9 & 28.2 & 29.7 & 31.0 & 32.1 \\
\hline ESF-HD-160-TEMP15-RTD-52 & -17.58 & 22.93 & 0.08 & 17.58 & 23.6 & 23.6 & 23.7 & 24.3 & 24.3 & 26.2 & 27.3 & 28.6 & 29.8 & 30.8 \\
\hline ESF-HD-160-TEMP15-RTD-53 & -17.88 & 22.94 & 0.08 & 17.88 & 23.4 & 23.5 & 23.7 & 24.1 & 24.1 & 25.6 & 26.5 & 27.7 & 28.7 & 29.6 \\
\hline ESF-HD-160-TEMP15-RTD-54 & -18.18 & 22.94 & 0.08 & 18.18 & 23.6 & 23.6 & 23.7 & 24.0 & 24.0 & 25.2 & 26.0 & 27.0 & 27.9 & 28.7 \\
\hline ESF-HD-160-TEMP15-RTD-55 & -18.48 & 22.94 & 0.08 & 18.48 & 23.7 & 23.7 & 23.7 & 23.9 & 23.9 & 24.9 & 25.5 & 26.3 & 27.1 & 27.8 \\
\hline ESF-HD-160-TEMP15-RTD-56 & -18.78 & 22.94 & 0.09 & 18.78 & 23.5 & 23.7 & 23.7 & 23.8 & 23.9 & 24.6 & 25.1 & 25.8 & 26.5 & 27.1 \\
\hline ESF-HD-160-TEMP15-RTD-57 & -19.08 & 22.94 & 0.09 & 19.08 & 23.5 & 23.6 & 49.1 & 23.8 & 66.5 & 24.3 & 24.8 & 25.4 & 26.0 & 26.5 \\
\hline
\end{tabular}




\begin{tabular}{|c|c|c|c|c|c|c|c|c|c|c|c|c|c|c|}
\hline & \multicolumn{14}{|c|}{ Temperature Data Obtained from the DST } \\
\hline & \multicolumn{14}{|c|}{ Days after startup } \\
\hline & \multicolumn{4}{|c|}{ As Built Locations (m) } & 0 & 7 & 17 & 27 & 38 & 48 & 58 & 69 & 79 & 87 \\
\hline & $\mathbf{x}$ & $\mathbf{y}$ & $\mathbf{z}$ & D & \multicolumn{10}{|c|}{$\left({ }^{\circ} \mathbf{C}\right)$} \\
\hline ESF-HD-160-TEMP15-RTD-58 & -19.38 & 22.94 & 0.09 & 19.38 & 23.6 & 23.6 & 23.7 & 23.8 & 23.8 & 24.2 & 24.6 & 25.1 & 25.6 & 26.0 \\
\hline ESF-HD-160-TEMP15-RTD-59 & -19.68 & 22.94 & 0.09 & 19.68 & 23.5 & 23.6 & 23.7 & 23.7 & 23.8 & 24.1 & 24.4 & 24.8 & 25.2 & 25.6 \\
\hline ESF-HD-160-TEMP15-RTD-60 & -19.99 & 22.94 & 0.09 & 19.99 & 23.6 & 23.6 & 23.7 & 23.7 & 23.8 & 24.0 & 24.3 & 24.6 & 25.0 & 25.3 \\
\hline ESF-HD-160-TEMP15-RTD-61 & -20.29 & 22.95 & 0.09 & 20.29 & 23.7 & 23.6 & 23.7 & 23.7 & 23.8 & 24.0 & 24.2 & 24.5 & 24.8 & 25.1 \\
\hline ESF-HD-160-TEMP15-RTD-62 & -20.59 & 22.95 & 0.10 & 20.59 & 23.7 & 23.6 & 23.7 & 23.8 & 23.8 & 23.9 & 24.1 & 24.3 & 24.6 & 24.9 \\
\hline ESF-HD-160-TEMP15-RTD-63 & -20.89 & 22.95 & 0.10 & 20.89 & 23.5 & 23.7 & 23.7 & 23.8 & 23.8 & 23.9 & 24.0 & 24.2 & 24.5 & 24.7 \\
\hline ESF-HD-160-TEMP15-RTD-64 & -21.19 & 22.95 & 0.10 & 21.19 & 23.6 & 23.7 & 23.8 & 23.8 & 23.9 & 23.9 & 24.0 & 24.2 & 24.3 & 24.5 \\
\hline ESF-HD-160-TEMP15-RTD-65 & -21.49 & 22.95 & 0.10 & 21.49 & 23.7 & 23.5 & 23.8 & 23.8 & 23.9 & 23.9 & 24.0 & 24.1 & 24.3 & 24.4 \\
\hline ESF-HD-160-TEMP15-RTD-66 & -21.79 & 22.95 & 0.10 & 21.79 & 23.6 & 23.7 & 48.6 & 23.8 & 66.4 & 23.9 & 24.0 & 24.1 & 24.2 & 24.3 \\
\hline ESF-HD-160-TEMP15-RTD-67 & -22.09 & 22.95 & 0.10 & 22.09 & 23.5 & 23.3 & 49.1 & 23.8 & 67.2 & 23.9 & 23.9 & 24.0 & 24.0 & 24.2 \\
\hline \multicolumn{15}{|l|}{ BH 161 Gage ID } \\
\hline ESF-HD-161-TEMP16-RTD-1 & -1.27 & 22.87 & -1.28 & 1.80 & 30.2 & 46.1 & 56.2 & 63.8 & 70.3 & 75.6 & 80.1 & 84.7 & 88.7 & 91.3 \\
\hline ESF-HD-161-TEMP16-RTD-2 & -1.49 & 22.88 & -1.49 & 2.11 & 30.2 & 42.7 & 52.2 & 59.8 & 66.3 & 71.6 & 76.3 & 80.8 & 84.8 & 87.4 \\
\hline ESF-HD-161-TEMP16-RTD-3 & -1.71 & 22.89 & -1.71 & 2.42 & 29.9 & 38.1 & 46.7 & 54.1 & 60.4 & 65.6 & 70.2 & 74.8 & 78.6 & 81.3 \\
\hline ESF-HD-161-TEMP16-RTD-4 & -1.93 & 22.89 & -1.92 & 2.72 & 29.9 & 35.0 & 42.7 & 49.5 & 55.7 & 60.8 & 65.4 & 70.0 & 73.8 & 76.6 \\
\hline ESF-HD-161-TEMP16-RTD-5 & -2.15 & 22.90 & -2.14 & 3.03 & 29.7 & 32.6 & 39.1 & 45.2 & 51.0 & 56.0 & 60.5 & 65.1 & 68.9 & 71.7 \\
\hline ESF-HD-161-TEMP16-RTD-6 & -2.37 & 22.90 & -2.36 & 3.34 & 29.5 & 31.0 & 36.3 & 41.7 & 47.3 & 52.0 & 56.4 & 60.9 & 64.7 & 67.6 \\
\hline ESF-HD-161-TEMP16-RTD-7 & -2.58 & 22.91 & -2.57 & 3.64 & 29.0 & 29.7 & 33.9 & 38.6 & 43.7 & 48.2 & 52.4 & 56.8 & 60.6 & 63.4 \\
\hline ESF-HD-161-TEMP16-RTD-8 & -2.80 & 22.91 & -2.79 & 3.95 & 28.7 & 29.0 & 32.1 & 36.3 & 41.0 & 45.3 & 49.4 & 53.6 & 57.3 & 60.2 \\
\hline ESF-HD-161-TEMP16-RTD-9 & -3.02 & 22.92 & -3.00 & 4.26 & 28.5 & 28.5 & 30.7 & 34.2 & 38.5 & 42.5 & 46.3 & 50.5 & 54.0 & 56.8 \\
\hline ESF-HD-161-TEMP16-RTD-10 & -3.24 & 22.92 & -3.22 & 4.57 & 28.0 & 27.9 & 29.7 & 32.7 & 36.6 & 40.3 & 43.9 & 47.9 & 51.4 & 54.1 \\
\hline ESF-HD-161-TEMP16-RTD-11 & -3.46 & 22.93 & -3.44 & 4.88 & 27.7 & 27.7 & 28.8 & 31.3 & 34.7 & 38.1 & 41.5 & 45.3 & 48.6 & 51.3 \\
\hline ESF-HD-161-TEMP16-RTD-12 & -3.68 & 22.93 & -3.65 & 5.18 & 27.3 & 27.3 & 28.1 & 30.1 & 33.1 & 36.3 & 39.5 & 43.1 & 46.3 & 48.9 \\
\hline ESF-HD-161-TEMP16-RTD-13 & -3.90 & 22.94 & -3.87 & 5.49 & 27.2 & 27.1 & 27.6 & 29.2 & 31.8 & 34.6 & 37.6 & 41.0 & 44.0 & 46.5 \\
\hline ESF-HD-161-TEMP16-RTD-14 & -4.11 & 22.95 & -4.08 & 5.79 & 26.7 & 26.7 & 27.1 & 28.4 & 30.6 & 33.1 & 35.9 & 39.0 & 41.9 & 44.3 \\
\hline ESF-HD-161-TEMP16-RTD-15 & -4.33 & 22.95 & -4.30 & 6.10 & 26.6 & 26.6 & 26.8 & 27.8 & 29.7 & 31.9 & 34.4 & 37.4 & 40.1 & 42.4 \\
\hline ESF-HD-161-TEMP16-RTD-16 & -4.55 & 22.96 & -4.52 & 6.41 & 26.2 & 26.3 & 26.5 & 27.3 & 28.9 & 30.9 & 33.2 & 35.9 & 38.5 & 40.6 \\
\hline ESF-HD-161-TEMP16-RTD-17 & -4.77 & 22.96 & -4.73 & 6.72 & 26.0 & 26.0 & 26.2 & 26.8 & 28.2 & 29.9 & 31.9 & 34.4 & 36.8 & 38.8 \\
\hline ESF-HD-161-TEMP16-RTD-18 & -4.99 & 22.97 & -4.95 & 7.03 & 25.8 & 25.9 & 26.0 & 26.5 & 27.6 & 29.1 & 31.0 & 33.3 & 35.6 & 37.5 \\
\hline ESF-HD-161-TEMP16-RTD-19 & -5.21 & 22.97 & -5.17 & 7.34 & 25.6 & 25.6 & 25.7 & 26.1 & 27.0 & 28.3 & 30.0 & 32.1 & 34.2 & 36.0 \\
\hline ESF-HD-161-TEMP16-RTD-20 & -5.43 & 22.98 & -5.38 & 7.64 & 25.4 & 25.5 & 25.6 & 25.8 & 26.6 & 27.7 & 29.2 & 31.1 & 33.1 & 34.7 \\
\hline ESF-HD-161-TEMP16-RTD-21 & -5.64 & 22.98 & -5.60 & 7.95 & 24.9 & 25.0 & 25.3 & 25.6 & 26.2 & 27.1 & 28.4 & 30.2 & 31.9 & 33.5 \\
\hline ESF-HD-161-TEMP16-RTD-22 & -5.86 & 22.99 & -5.81 & 8.25 & 25.0 & 25.0 & 25.2 & 25.4 & 25.9 & 26.7 & 27.8 & 29.4 & 31.0 & 32.4 \\
\hline ESF-HD-161-TEMP16-RTD-23 & -6.08 & 22.99 & -6.03 & 8.56 & 24.8 & 24.7 & 25.0 & 25.2 & 25.6 & 26.3 & 27.3 & 28.7 & 30.1 & 31.5 \\
\hline ESF-HD-161-TEMP16-RTD-24 & -6.30 & 23.00 & -6.25 & 8.87 & 24.8 & 24.8 & 24.9 & 25.1 & 25.4 & 26.0 & 26.9 & 28.1 & 29.5 & 30.7 \\
\hline ESF-HD-161-TEMP16-RTD-25 & -6.52 & 23.00 & -6.46 & 9.18 & 24.7 & 24.7 & 24.8 & 24.9 & 25.2 & 25.7 & 26.5 & 27.5 & 28.8 & 29.8 \\
\hline ESF-HD-161-TEMP16-RTD-26 & -6.74 & 23.01 & -6.68 & 9.49 & 24.6 & 24.6 & 24.7 & 24.7 & 25.0 & 25.4 & 26.0 & 27.0 & 28.1 & 29.1 \\
\hline
\end{tabular}


First Quarter Report of Temperature Measurements During the Heating Phase of the Drift-Scale Test

\begin{tabular}{|c|c|c|c|c|c|c|c|c|c|c|c|c|c|c|}
\hline \multirow{5}{*}{\begin{tabular}{|l|} 
\\
ESF-HD-161-TEMP16-RTD-27 \\
\end{tabular}} & \multicolumn{14}{|c|}{ Temperature Data Obtained from the DST } \\
\hline & \multicolumn{14}{|c|}{ Days after startup } \\
\hline & \multicolumn{4}{|c|}{ As Built Locations (m) } & 0 & 7 & 17 & 27 & 38 & 48 & 58 & 69 & 79 & 87 \\
\hline & $\mathbf{x}$ & $\mathbf{y}$ & $\mathbf{z}$ & D & \multicolumn{10}{|c|}{$\left({ }^{\circ} \mathbf{C}\right)$} \\
\hline & -6.96 & 23.02 & -6.89 & 9.79 & 24.5 & 24.5 & 24.6 & 24.6 & 24.8 & 25.0 & 25.5 & 26.5 & 27.4 & 28.4 \\
\hline ESF-HD-161-TEMP16-RTD-28 & -7.17 & 23.02 & -7.11 & 10.10 & 24.4 & 24.3 & 24.5 & 24.6 & 24.7 & 25.0 & 25.5 & 26.2 & 27.1 & 27.9 \\
\hline ESF-HD-161-TEMP16-RTD-29 & -7.39 & 23.03 & -7.33 & 10.41 & 24.2 & 24.3 & 24.4 & 24.5 & 24.6 & 24.8 & 25.2 & 25.8 & 26.5 & 27.3 \\
\hline ESF-HD-161-TEMP16-RTD-30 & -7.61 & 23.03 & -7.54 & 10.71 & 24.2 & 24.2 & 24.3 & 24.4 & 24.5 & 24.7 & 25.0 & 25.5 & 26.2 & 26.9 \\
\hline ESF-HD-161-TEMP16-RTD-31 & -7.83 & 23.04 & -7.76 & 11.02 & 24.1 & 24.1 & 24.2 & 24.3 & 24.4 & 24.5 & 24.8 & 25.3 & 25.8 & 26.4 \\
\hline ESF-HD-161-TEMP16-RTD-32 & -8.05 & 23.04 & -7.97 & 11.33 & 24.1 & 24.0 & 24.2 & 24.3 & 24.3 & 24.5 & 24.7 & 25.1 & 25.6 & 26.1 \\
\hline ESF-HD-161-TEMP16-RTD-33 & -8.27 & 23.05 & -8.19 & 11.64 & 24.1 & 24.1 & 24.2 & 24.2 & 24.3 & 24.4 & 24.6 & 24.9 & 25.3 & 25.8 \\
\hline ESF-HD-161-TEMP16-RTD-34 & -8.49 & 23.05 & -8.41 & 11.95 & 24.1 & 24.1 & 24.1 & 24.2 & 24.2 & 24.3 & 24.4 & 24.7 & 25.1 & 25.5 \\
\hline ESF-HD-161-TEMP16-RTD-35 & -8.70 & 23.06 & -8.62 & 12.25 & 24.0 & 24.0 & 24.1 & 24.1 & 24.1 & 24.2 & 24.3 & 24.5 & 24.9 & 25.2 \\
\hline ESF-HD-161-TEMP16-RTD-36 & -8.92 & 23.06 & -8.84 & 12.56 & 23.9 & 23.9 & 24.0 & 24.0 & 24.1 & 24.1 & 24.2 & 24.5 & 24.7 & 25.0 \\
\hline ESF-HD-161-TEMP16-RTD-37 & -9.14 & 23.07 & -9.05 & 12.86 & 23.9 & 24.0 & 24.0 & 24.0 & 24.1 & 24.1 & 24.2 & 24.4 & 24.6 & 24.8 \\
\hline ESF-HD-161-TEMP16-RTD-38 & -9.36 & 23.08 & -9.27 & 13.17 & 23.8 & 23.8 & 23.9 & 23.9 & 23.9 & 24.0 & 24.0 & 24.2 & 24.4 & 24.6 \\
\hline ESF-HD-161-TEMP16-RTD-39 & -9.58 & 23.08 & -9.49 & 13.49 & 23.8 & 23.9 & 24.0 & 24.0 & 24.0 & 24.0 & 24.1 & 24.2 & 24.4 & 24.6 \\
\hline ESF-HD-161-TEMP16-RTD-40 & -9.80 & 23.09 & -9.70 & 13.79 & 23.8 & 23.7 & 23.9 & 23.9 & 23.9 & 24.0 & 24.0 & 24.1 & 24.2 & 24.4 \\
\hline ESF-HD-161-TEMP16-RTD-41 & -10.02 & 23.09 & -9.92 & 14.10 & 23.8 & 23.8 & 23.8 & 23.9 & 23.9 & 23.9 & 23.9 & 24.0 & 24.1 & 24.3 \\
\hline ESF-HD-161-TEMP16-RTD-42 & -10.23 & 23.10 & -10.14 & 14.40 & 23.7 & 23.8 & 23.9 & 23.9 & 23.9 & 23.9 & 24.0 & 24.0 & 24.1 & 24.2 \\
\hline ESF-HD-161-TEMP16-RTD-43 & -10.45 & 23.10 & -10.35 & 14.71 & 23.7 & 23.8 & 23.9 & 23.9 & 23.9 & 23.9 & 23.9 & 24.0 & 24.1 & 24.2 \\
\hline ESF-HD-161-TEMP16-RTD-44 & -10.67 & 23.11 & -10.57 & 15.02 & 23.8 & 23.8 & 23.8 & 23.8 & 23.8 & 23.9 & 23.9 & 23.9 & 24.0 & 24.1 \\
\hline ESF-HD-161-TEMP16-RTD-45 & -10.89 & 23.11 & -10.78 & 15.32 & 23.7 & 23.7 & 23.8 & 23.8 & 23.8 & 23.8 & 23.8 & 23.9 & 23.9 & 24.0 \\
\hline ESF-HD-161-TEMP16-RTD-46 & -11.11 & 23.12 & -11.00 & 15.63 & 23.7 & 23.7 & 23.7 & 23.8 & 23.8 & 23.8 & 23.8 & 23.8 & 23.9 & 23.9 \\
\hline ESF-HD-161-TEMP16-RTD-47 & -11.33 & 23.12 & -11.22 & 15.95 & 23.8 & 23.8 & 23.8 & 23.8 & 23.8 & 23.8 & 23.8 & 23.8 & 23.9 & 23.9 \\
\hline ESF-HD-161-TEMP16-RTD-48 & -11.55 & 23.13 & -11.43 & 16.25 & 23.6 & 23.7 & 23.8 & 23.8 & 23.8 & 23.8 & 23.8 & 23.9 & 23.9 & 23.9 \\
\hline ESF-HD-161-TEMP16-RTD-49 & -11.76 & 23.13 & -11.65 & 16.55 & 23.7 & 23.7 & 23.8 & 23.8 & 23.8 & 23.8 & 23.8 & 23.8 & 23.8 & 23.9 \\
\hline ESF-HD-161-TEMP16-RTD-50 & -11.98 & 23.14 & -11.86 & 16.86 & 22.1 & 23.0 & 23.6 & 23.7 & 23.7 & 23.7 & 23.7 & 23.8 & 23.8 & 23.8 \\
\hline ESF-HD-161-TEMP16-RTD-51 & -12.20 & 23.15 & -12.08 & 17.17 & 23.8 & 23.7 & 23.8 & 23.8 & 23.8 & 23.8 & 23.8 & 23.8 & 23.8 & 23.8 \\
\hline ESF-HD-161-TEMP16-RTD-52 & -12.42 & 23.15 & -12.30 & 17.48 & 23.7 & 23.7 & 23.8 & 23.8 & 23.8 & 23.8 & 23.8 & 23.8 & 23.8 & 23.9 \\
\hline ESF-HD-161-TEMP16-RTD-53 & -12.64 & 23.16 & -12.51 & 17.78 & 23.6 & 23.6 & 23.7 & 23.7 & 23.7 & 23.7 & 23.7 & 23.8 & 23.8 & 23.8 \\
\hline ESF-HD-161-TEMP16-RTD-54 & -12.86 & 23.16 & -12.73 & 18.10 & 23.7 & 23.6 & 23.7 & 23.7 & 23.7 & 23.7 & 23.7 & 23.7 & 23.7 & 23.8 \\
\hline ESF-HD-161-TEMP16-RTD-55 & -13.08 & 23.17 & -12.94 & 18.40 & 23.7 & 23.5 & 23.7 & 23.7 & 23.7 & 23.7 & 23.7 & 23.8 & 23.8 & 23.8 \\
\hline ESF-HD-161-TEMP16-RTD-56 & -13.29 & 23.17 & -13.16 & 18.70 & 23.6 & 23.5 & 23.6 & 23.6 & 23.6 & 23.6 & 23.6 & 23.7 & 23.7 & 23.7 \\
\hline ESF-HD-161-TEMP16-RTD-57 & -13.51 & 23.18 & -13.38 & 19.01 & 23.7 & 23.6 & 23.7 & 23.7 & 23.7 & 23.7 & 23.7 & 23.7 & 23.7 & 23.8 \\
\hline ESF-HD-161-TEMP16-RTD-58 & -13.73 & 23.18 & -13.59 & 19.32 & 23.7 & 23.7 & 23.7 & 23.7 & 23.6 & 23.7 & 23.7 & 23.7 & 23.7 & 23.7 \\
\hline ESF-HD-161-TEMP16-RTD-59 & -13.95 & 23.19 & -13.81 & 19.63 & 23.7 & 23.7 & 23.7 & 23.7 & 23.7 & 23.7 & 23.7 & 23.7 & 23.7 & 23.7 \\
\hline ESF-HD-161-TEMP16-RTD-60 & -14.17 & 23.19 & -14.02 & 19.93 & 23.7 & 23.5 & 23.7 & 23.7 & 23.7 & 23.7 & 23.7 & 23.7 & 23.7 & 23.7 \\
\hline ESF-HD-161-TEMP16-RTD-61 & -14.39 & 23.20 & -14.24 & 20.25 & 23.7 & 23.6 & 23.7 & 23.7 & 23.7 & 23.7 & 23.7 & 23.7 & 23.7 & 23.7 \\
\hline ESF-HD-161-TEMP16-RTD-62 & -14.61 & 23.21 & -14.46 & 20.56 & 23.6 & 23.5 & 23.6 & 23.6 & 23.6 & 23.6 & 23.6 & 23.7 & 23.6 & 23.6 \\
\hline ESF-HD-161-TEMP16-RTD-63 & -14.82 & 23.21 & -14.67 & 20.85 & 23.7 & 23.6 & 23.7 & 23.7 & 23.7 & 23.7 & 23.7 & 23.7 & 23.7 & 23.7 \\
\hline
\end{tabular}




\begin{tabular}{|c|c|c|c|c|c|c|c|c|c|c|c|c|c|c|}
\hline & \multicolumn{14}{|c|}{ Temperature Data Obtained from the DST } \\
\hline & \multicolumn{14}{|c|}{ Days after startup } \\
\hline & \multicolumn{4}{|c|}{ As Built Locations (m) } & 0 & 7 & 17 & 27 & 38 & 48 & 58 & 69 & 79 & 87 \\
\hline & $\mathbf{x}$ & $\mathbf{y}$ & $\mathbf{z}$ & D & \multicolumn{10}{|c|}{$\left({ }^{\circ} \mathbf{C}\right)$} \\
\hline ESF-HD-161-TEMP16-RTD-64 - & -15.04 & 23.22 & -14.89 & 21.16 & 23.7 & 23.6 & 23.7 & 23.7 & 23.7 & 23.7 & 23.7 & 23.7 & 23.7 & 23.5 \\
\hline ESF-HD-161-TEMP16-RTD-65 - & -15.26 & 23.22 & -15.11 & 21.48 & 23.7 & 23.7 & 23.7 & 23.7 & 23.7 & 23.7 & 23.7 & 23.7 & 23.7 & 23.7 \\
\hline ESF-HD-161-TEMP16-RTD-66 - & -15.48 & 23.23 & -15.32 & 21.78 & 23.7 & 23.7 & 23.7 & 23.7 & 23.7 & 23.7 & 23.7 & 23.7 & 23.7 & 23.7 \\
\hline ESF-HD-161-TEMP16-RTD-67 - & -15.70 & 23.23 & -15.54 & 22.09 & 23.7 & 23.6 & 23.7 & 23.7 & 23.7 & 23.7 & 23.7 & 23.7 & 23.7 & 23.7 \\
\hline \multicolumn{15}{|l|}{ BH 162 Gage ID } \\
\hline ESF-HD-162-TEMP17-RTD-1 & 0.77 & 22.85 & -1.37 & 1.37 & 30.0 & 49.7 & 60.2 & 67.6 & & 79.4 & 84.1 & 88.5 & 92.3 & 94.9 \\
\hline ESF-HD-162-TEMP17-RTD-2 & 0.77 & 22.85 & -1.67 & 1.67 & 30.3 & 43.9 & 54.1 & 61.3 & 74.2 & 72.8 & 77.4 & 82.0 & 85.7 & 88.3 \\
\hline ESF-HD-162-TEMP17-RTD-3 & 0.77 & 22.85 & -1.97 & 1.97 & 30.4 & 39.3 & 48.8 & 55.7 & 35.3 & 67.0 & 71.5 & 76.0 & 79.7 & 82.3 \\
\hline ESF-HD-162-TEMP17-RTD-4 & 0.77 & 22.85 & -2.27 & 2.27 & 30.4 & 35.7 & 44.2 & 50.7 & 33.4 & 61.5 & 65.9 & 70.3 & 73.9 & 76.6 \\
\hline ESF-HD-162-TEMP17-RTD-5 & 0.77 & 22.86 & -2.57 & 2.57 & 30.1 & 33.2 & 40.4 & 46.3 & 31.9 & 56.5 & 60.7 & 65.0 & 68.5 & 71.2 \\
\hline ESF-HD-162-TEMP17-RTD-6 & 0.77 & 22.86 & -2.87 & 2.87 & 29.7 & 31.3 & 37.1 & 42.4 & 30.5 & 51.8 & 55.8 & 59.9 & 63.4 & 66.0 \\
\hline ESF-HD-162-TEMP17-RTD-7 & 0.77 & 22.86 & -3.16 & 3.16 & 29.6 & 30.2 & 34.5 & 39.0 & 29.6 & 47.6 & 51.4 & 55.3 & 58.6 & 61.2 \\
\hline ESF-HD-162-TEMP17-RTD-8 & 0.77 & 22.86 & -3.46 & 3.46 & 29.0 & 29.2 & 32.5 & 36.3 & 28.6 & 44.0 & 47.5 & 51.2 & 54.4 & 56.8 \\
\hline ESF-HD-162-TEMP17-RTD-9 & 0.78 & 22.87 & -3.76 & 3.76 & 28.9 & 28.9 & 31.0 & 34.1 & 28.0 & 40.9 & 44.1 & 47.6 & 50.6 & 52.9 \\
\hline ESF-HD-162-TEMP17-RTD-10 & 0.78 & 22.87 & -4.06 & 4.06 & 28.3 & 27.9 & 29.8 & 32.4 & 27.4 & 38.2 & 41.1 & 44.5 & 47.1 & 49.5 \\
\hline ESF-HD-162-TEMP17-RTD-11 & 0.78 & 22.87 & -4.36 & 4.36 & 28.2 & 28.1 & 28.9 & 30.8 & 26.9 & 35.9 & 38.5 & 41.4 & 44.0 & 46.0 \\
\hline ESF-HD-162-TEMP17-RTD-12 & 0.78 & 22.87 & -4.66 & 4.66 & 27.6 & 27.5 & 28.2 & 29.7 & 26.5 & 34.1 & 36.4 & 39.0 & 41.4 & 43.3 \\
\hline ESF-HD-162-TEMP17-RTD-13 & 0.78 & 22.87 & -4.96 & 4.96 & 27.4 & 27.4 & 27.7 & 28.8 & 67.7 & 32.4 & 34.5 & 36.8 & 39.0 & 40.8 \\
\hline ESF-HD-162-TEMP17-RTD-14 & 0.78 & 22.88 & -5.26 & 5.26 & 27.1 & 26.6 & 27.3 & 28.2 & 26.3 & 31.1 & 32.7 & 35.0 & 37.0 & 38.7 \\
\hline ESF-HD-162-TEMP17-RTD-15 & 0.78 & 22.88 & -5.56 & 5.56 & 26.8 & 26.9 & 27.0 & 27.5 & 25.8 & 29.9 & 31.4 & 33.3 & 35.0 & 36.5 \\
\hline ESF-HD-162-TEMP17-RTD-16 & 0.78 & 22.88 & -5.86 & 5.86 & 26.6 & 26.6 & 26.7 & 27.1 & 25.6 & 29.1 & 30.4 & 32.0 & 33.6 & 35.0 \\
\hline ESF-HD-162-TEMP17-RTD-17 & 0.78 & 22.88 & -6.16 & 6.16 & 26.3 & 26.3 & 26.4 & 26.7 & 25.5 & 28.2 & 29.3 & 30.7 & 32.1 & 33.3 \\
\hline ESF-HD-162-TEMP17-RTD-18 & 0.78 & 22.88 & -6.45 & 6.45 & 25.8 & 26.0 & 26.2 & 26.4 & 25.3 & 27.6 & 28.5 & 29.8 & 31.0 & 32.1 \\
\hline ESF-HD-162-TEMP17-RTD-19 & 0.78 & 22.89 & -6.75 & 6.75 & 25.8 & 25.9 & 26.0 & 26.1 & 25.1 & 27.1 & 27.9 & 28.9 & 30.0 & 30.9 \\
\hline ESF-HD-162-TEMP17-RTD-20 & 0.78 & 22.89 & -7.05 & 7.05 & 25.5 & 25.7 & 25.9 & 26.0 & 24.9 & 26.8 & 27.4 & 28.3 & 29.2 & 30.1 \\
\hline ESF-HD-162-TEMP17-RTD-21 & 0.79 & 22.89 & -7.35 & 7.35 & 25.4 & 25.5 & 25.5 & 25.6 & 24.8 & 26.2 & 26.7 & 27.4 & 28.2 & 29.0 \\
\hline ESF-HD-162-TEMP17-RTD-22 & 0.79 & 22.89 & -7.65 & 7.65 & 25.2 & 25.2 & 25.4 & 25.5 & 24.7 & 25.9 & 26.3 & 26.9 & 27.6 & 28.2 \\
\hline ESF-HD-162-TEMP17-RTD-23 & 0.79 & 22.90 & -7.95 & 7.95 & 25.0 & 25.1 & 25.2 & 25.3 & 24.6 & 25.7 & 26.0 & 26.5 & 27.1 & 27.6 \\
\hline ESF-HD-162-TEMP17-RTD-24 & 0.79 & 22.90 & -8.25 & 8.25 & 24.8 & 24.9 & 25.1 & 25.2 & 62.0 & 25.5 & 25.7 & 26.1 & 26.6 & 27.1 \\
\hline ESF-HD-162-TEMP17-RTD-25 & 0.79 & 22.90 & -8.55 & 8.55 & 24.8 & 24.8 & 24.9 & 25.0 & 24.4 & 25.2 & 25.4 & 25.7 & 26.1 & 26.5 \\
\hline ESF-HD-162-TEMP17-RTD-26 & 0.79 & 22.90 & -8.85 & 8.85 & 24.7 & 24.7 & 24.8 & 24.8 & 24.4 & 25.0 & 25.2 & 25.4 & 25.8 & 26.1 \\
\hline ESF-HD-162-TEMP17-RTD-27 & 0.79 & 22.90 & -9.15 & 9.15 & 24.5 & 24.5 & 24.7 & 24.8 & 24.4 & 24.9 & 25.0 & 25.3 & 25.5 & 25.8 \\
\hline ESF-HD-162-TEMP17-RTD-28 & 0.79 & 22.91 & -9.45 & 9.45 & 24.3 & 24.4 & 24.6 & 24.6 & 24.3 & 24.8 & 24.9 & 25.1 & 25.3 & 25.5 \\
\hline ESF-HD-162-TEMP17-RTD-29 & 0.79 & 22.91 & -9.74 & 9.74 & 24.3 & 24.4 & 24.5 & 24.6 & 24.2 & 24.7 & 24.8 & 24.9 & 25.1 & 25.3 \\
\hline ESF-HD-162-TEMP17-RTD-30 & 0.79 & 22.91 & -10.04 & 10.04 & 24.1 & 24.0 & 24.3 & 24.4 & 24.2 & 24.5 & 24.5 & 24.7 & 24.8 & 25.0 \\
\hline ESF-HD-162-TEMP17-RTD-31 & 0.79 & 22.91 & -10.34 & 10.34 & 24.2 & 24.1 & 24.3 & 24.3 & 24.1 & 24.4 & 24.5 & 24.6 & 24.7 & 24.8 \\
\hline ESF-HD-162-TEMP17-RTD-32 & 0.79 & 22.92 & -10.64 & 10.64 & 24.1 & 24.1 & 24.3 & 24.3 & 24.1 & 24.4 & 24.5 & 24.5 & 24.6 & 24.7 \\
\hline
\end{tabular}


First Quarter Report of Temperature Measurements During the Heating Phase of the Drift-Scale Test

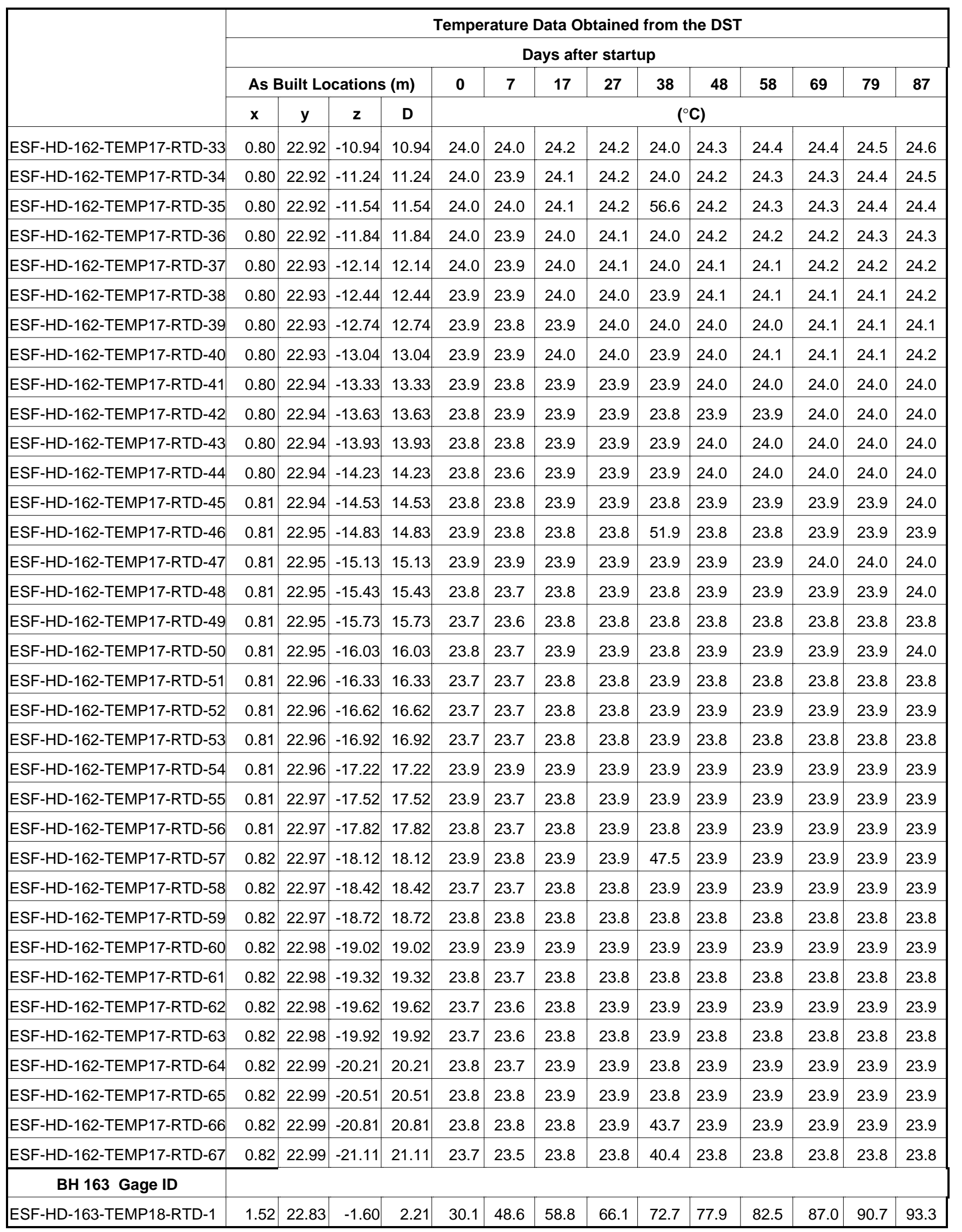




\begin{tabular}{|c|c|c|c|c|c|c|c|c|c|c|c|c|c|c|}
\hline & \multicolumn{14}{|c|}{ Temperature Data Obtained from the DST } \\
\hline & \multicolumn{14}{|c|}{ Days after startup } \\
\hline & \multicolumn{4}{|c|}{ As Built Locations (m) } & 0 & 7 & 17 & 27 & 38 & 48 & 58 & 69 & 79 & 87 \\
\hline & $\mathbf{x}$ & $\mathbf{y}$ & $\mathbf{z}$ & D & \multicolumn{10}{|c|}{$\left({ }^{\circ} \mathbf{C}\right)$} \\
\hline ESF-HD-163-TEMP18-RTD-2 & 1.73 & 22.82 & -1.81 & 2.50 & 30.0 & 41.5 & 51.2 & 58.3 & 64.8 & 69.9 & 74.5 & 79.1 & 82.9 & 85.5 \\
\hline ESF-HD-163-TEMP18-RTD-3 & 1.94 & 22.82 & -2.02 & 2.80 & 30.0 & 37.6 & 46.4 & 53.2 & 59.5 & 64.5 & 69.1 & 73.7 & 77.4 & 80.1 \\
\hline ESF-HD-163-TEMP18-RTD-4 & 2.15 & 22.81 & -2.24 & 3.10 & 29.8 & 34.7 & 42.4 & 48.8 & 54.9 & 59.9 & 64.4 & 69.0 & 72.8 & 75.6 \\
\hline ESF-HD-163-TEMP18-RTD-5 & 2.36 & 22.81 & -2.45 & 3.40 & 29.7 & 32.2 & 38.6 & 44.5 & 50.3 & 55.2 & 59.7 & 64.2 & 68.0 & 70.8 \\
\hline ESF-HD-163-TEMP18-RTD-6 & 2.57 & 22.80 & -2.66 & 3.70 & 29.4 & 30.8 & 35.9 & 41.2 & 46.7 & 51.4 & 55.7 & 60.2 & 63.9 & 66.8 \\
\hline ESF-HD-163-TEMP18-RTD-7 & 2.79 & 22.80 & -2.87 & 4.00 & 29.0 & 29.6 & 33.5 & 38.2 & 43.2 & 47.6 & 51.8 & 56.1 & 59.7 & 62.6 \\
\hline ESF-HD-163-TEMP18-RTD-8 & 3.00 & 22.79 & -3.09 & 4.31 & 28.5 & 28.8 & 31.8 & 35.9 & 40.6 & 44.7 & 48.8 & 53.0 & 56.6 & 59.4 \\
\hline ESF-HD-163-TEMP18-RTD-9 & 3.21 & 22.79 & -3.30 & 4.60 & 28.3 & 28.4 & 30.5 & 33.9 & 38.1 & 42.0 & 45.8 & 49.8 & 53.4 & 56.1 \\
\hline ESF-HD-163-TEMP18-RTD-10 & 3.42 & 22.79 & -3.51 & 4.90 & 27.9 & 27.8 & 29.4 & 32.3 & 36.1 & 39.7 & 43.3 & 47.2 & 50.7 & 53.3 \\
\hline ESF-HD-163-TEMP18-RTD-11 & 3.63 & 22.78 & -3.72 & 5.20 & 27.5 & 27.5 & 28.6 & 31.0 & 34.4 & 37.7 & 41.1 & 44.8 & 48.1 & 50.7 \\
\hline ESF-HD-163-TEMP18-RTD-12 & 3.85 & 22.78 & -3.94 & 5.51 & 27.2 & 27.1 & 28.0 & 29.9 & 32.9 & 35.9 & 39.1 & 42.6 & 45.8 & 48.3 \\
\hline ESF-HD-163-TEMP18-RTD-13 & 4.06 & 22.77 & -4.15 & 5.81 & 27.0 & 27.0 & 27.5 & 29.1 & 31.6 & 34.4 & 37.3 & 40.6 & 43.6 & 46.0 \\
\hline ESF-HD-163-TEMP18-RTD-14 & 4.27 & 22.77 & -4.36 & 6.10 & 26.8 & 26.8 & 27.1 & 28.3 & 30.6 & 33.0 & 35.7 & 38.8 & 41.7 & 44.0 \\
\hline ESF-HD-163-TEMP18-RTD-15 & 4.48 & 22.76 & -4.57 & 6.40 & 26.3 & 25.5 & 26.7 & 27.7 & 29.6 & 31.9 & 34.4 & 37.3 & 40.0 & 42.2 \\
\hline ESF-HD-163-TEMP18-RTD-16 & 4.69 & 22.76 & -4.79 & 6.70 & 26.2 & 26.3 & 26.4 & 27.2 & 28.8 & 30.8 & 33.0 & 35.7 & 38.3 & 40.4 \\
\hline ESF-HD-163-TEMP18-RTD-17 & 4.90 & 22.75 & -5.00 & 7.00 & 25.7 & 25.7 & 26.1 & 26.7 & 28.0 & 29.6 & 31.6 & 34.2 & 36.5 & 38.6 \\
\hline ESF-HD-163-TEMP18-RTD-18 & 5.12 & 22.75 & -5.21 & 7.30 & 25.7 & 25.8 & 25.9 & 26.4 & 27.5 & 29.0 & 30.8 & 33.1 & 35.3 & 37.2 \\
\hline ESF-HD-163-TEMP18-RTD-19 & 5.33 & 22.74 & -5.43 & 7.61 & 25.4 & 25.2 & 25.6 & 26.0 & 27.0 & 28.3 & 29.9 & 32.0 & 34.0 & 35.8 \\
\hline ESF-HD-163-TEMP18-RTD-20 & 5.54 & 22.74 & -5.64 & 7.91 & 25.3 & 25.4 & 25.5 & 25.8 & 26.6 & 27.7 & 29.1 & 31.0 & 32.9 & 34.6 \\
\hline ESF-HD-163-TEMP18-RTD-21 & 5.75 & 22.73 & -5.85 & 8.20 & 25.0 & 24.9 & 25.3 & 25.5 & 26.2 & 27.1 & 28.4 & 30.1 & 31.8 & 33.3 \\
\hline ESF-HD-163-TEMP18-RTD-22 & 5.96 & 22.73 & -6.06 & 8.50 & 25.0 & 25.1 & 25.2 & 25.4 & 25.9 & 26.8 & 27.9 & 29.4 & 31.0 & 32.4 \\
\hline ESF-HD-163-TEMP18-RTD-23 & 6.18 & 22.72 & -6.28 & 8.81 & 24.8 & 24.9 & 25.0 & 25.2 & 25.6 & 26.3 & 27.3 & 28.7 & 30.2 & 31.4 \\
\hline ESF-HD-163-TEMP18-RTD-24 & 6.39 & 22.72 & -6.49 & 9.11 & 24.7 & 24.8 & 24.9 & 25.0 & 25.4 & 26.0 & 26.8 & 28.0 & 29.3 & 30.5 \\
\hline ESF-HD-163-TEMP18-RTD-25 & 6.60 & 22.71 & -6.70 & 9.40 & 24.4 & 24.6 & 24.7 & 24.9 & 25.1 & 25.7 & 26.4 & 27.4 & 28.6 & 29.6 \\
\hline ESF-HD-163-TEMP18-RTD-26 & 6.81 & 22.71 & -6.91 & 9.70 & 24.5 & 24.6 & 24.7 & 24.8 & 25.0 & 25.4 & 26.1 & 27.0 & 28.1 & 29.0 \\
\hline ESF-HD-163-TEMP18-RTD-27 & 7.02 & 22.70 & -7.13 & 10.01 & 24.3 & 24.5 & 24.6 & 24.7 & 24.9 & 25.3 & 25.9 & 26.7 & 27.6 & 28.5 \\
\hline ESF-HD-163-TEMP18-RTD-28 & 7.23 & 22.70 & -7.34 & 10.30 & 24.2 & 24.2 & 24.3 & 24.4 & 24.5 & 24.8 & 25.2 & 25.8 & 26.5 & 27.2 \\
\hline ESF-HD-163-TEMP18-RTD-29 & 7.45 & 22.70 & -7.55 & 10.61 & 24.2 & 24.2 & 24.4 & 24.5 & 24.7 & 25.0 & 25.4 & 26.1 & 27.0 & 27.7 \\
\hline ESF-HD-163-TEMP18-RTD-30 & 7.66 & 22.69 & -7.76 & 10.90 & 24.2 & 24.2 & 24.3 & 24.4 & 24.4 & 24.7 & 25.0 & 25.5 & 26.2 & 26.8 \\
\hline ESF-HD-163-TEMP18-RTD-31 & 7.87 & 22.69 & -7.98 & 11.21 & 23.8 & 23.9 & 24.1 & 24.2 & 24.3 & 24.5 & 24.8 & 25.2 & 25.8 & 26.3 \\
\hline ESF-HD-163-TEMP18-RTD-32 & 8.08 & 22.68 & -8.19 & 11.51 & 24.1 & 24.1 & 24.1 & 24.2 & 24.3 & 24.4 & 24.7 & 25.0 & 25.5 & 26.0 \\
\hline ESF-HD-163-TEMP18-RTD-33 & 8.29 & 22.68 & -8.40 & 11.80 & 23.9 & 23.9 & 24.1 & 24.2 & 24.2 & 24.3 & 24.5 & 24.9 & 25.3 & 25.7 \\
\hline ESF-HD-163-TEMP18-RTD-34 & 8.51 & 22.67 & -8.62 & 12.11 & 23.9 & 23.9 & 24.0 & 24.1 & 24.2 & 24.3 & 24.4 & 24.7 & 25.1 & 25.5 \\
\hline ESF-HD-163-TEMP18-RTD-35 & 8.72 & 22.67 & -8.83 & 12.41 & 23.9 & 23.9 & 24.0 & 24.0 & 24.1 & 24.2 & 24.3 & 24.5 & 24.9 & 25.2 \\
\hline ESF-HD-163-TEMP18-RTD-36 & 8.93 & 22.66 & -9.04 & 12.71 & 23.9 & 23.9 & 24.0 & 24.0 & 24.1 & 24.1 & 24.3 & 24.5 & 24.7 & 25.0 \\
\hline ESF-HD-163-TEMP18-RTD-37 & 9.14 & 22.66 & -9.25 & 13.00 & 23.9 & 23.8 & 23.9 & 24.0 & 24.0 & 24.1 & 24.2 & 24.4 & 24.6 & 24.8 \\
\hline ESF-HD-163-TEMP18-RTD-38 & 9.35 & 22.65 & -9.47 & 13.31 & 23.8 & 23.9 & 24.0 & 24.0 & 24.0 & 24.1 & 24.2 & 24.3 & 24.5 & 24.7 \\
\hline
\end{tabular}


First Quarter Report of Temperature Measurements During the Heating Phase of the Drift-Scale Test

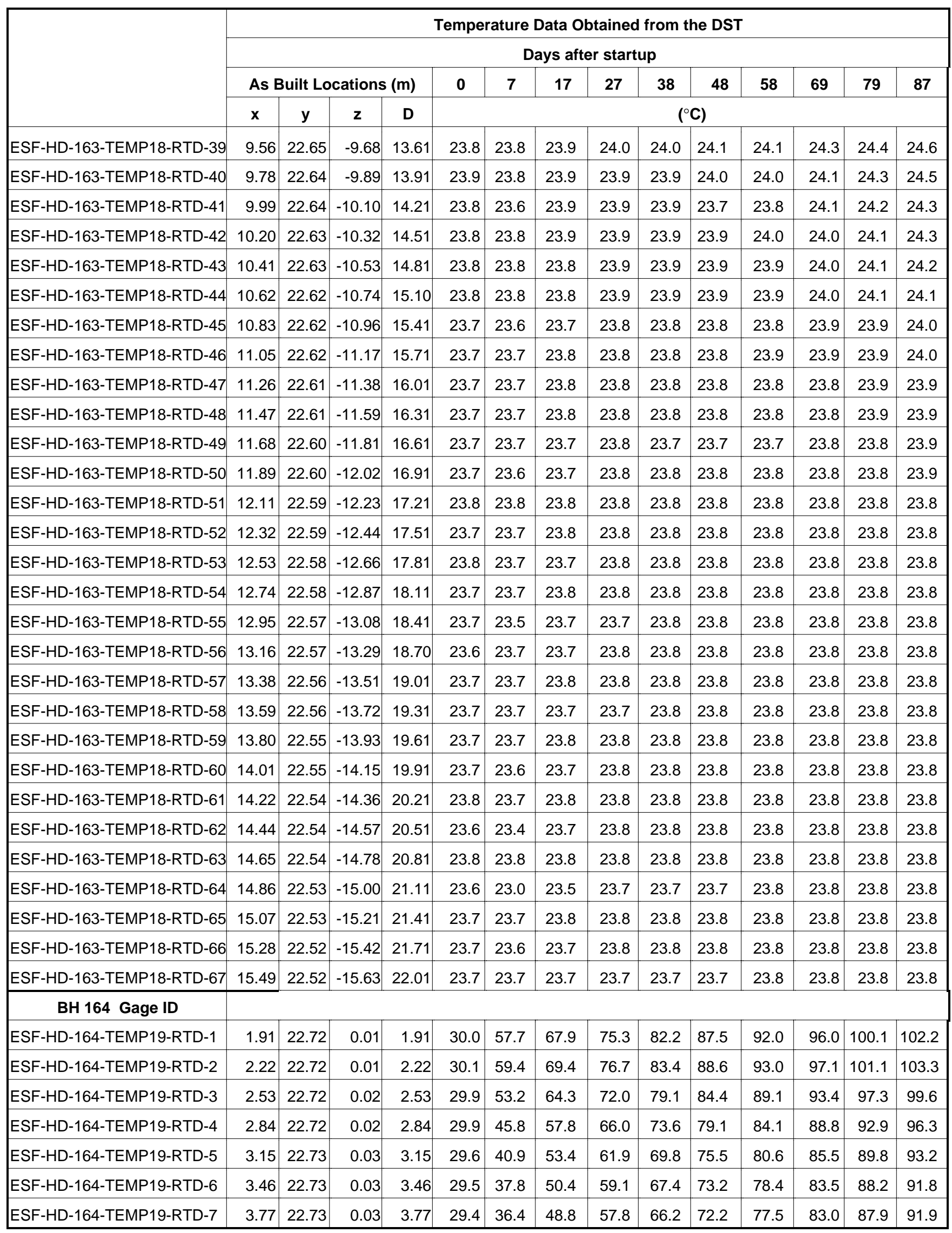




\begin{tabular}{|c|c|c|c|c|c|c|c|c|c|c|c|c|c|c|}
\hline & \multicolumn{14}{|c|}{ Temperature Data Obtained from the DST } \\
\hline & \multicolumn{14}{|c|}{ Days after startup } \\
\hline & \multicolumn{4}{|c|}{ As Built Locations (m) } & 0 & 7 & 17 & 27 & 38 & 48 & 58 & 69 & 79 & 87 \\
\hline & $\mathbf{x}$ & $\mathbf{y}$ & $\mathbf{z}$ & D & \multicolumn{10}{|c|}{$\left({ }^{\circ} \mathbf{C}\right)$} \\
\hline ESF-HD-164-TEMP19-RTD-8 & 4.07 & 22.73 & 0.04 & 4.07 & 29.0 & 35.7 & 48.1 & 57.4 & 65.9 & 72.2 & 77.8 & 83.6 & 89.0 & 93.2 \\
\hline ESF-HD-164-TEMP19-RTD-9 & 4.38 & 22.73 & 0.04 & 4.38 & 28.8 & 36.3 & 48.4 & 58.0 & 66.7 & 73.2 & 79.1 & 85.1 & 91.1 & 95.2 \\
\hline ESF-HD-164-TEMP19-RTD-10 & 4.69 & 22.74 & 0.05 & 4.69 & 28.4 & 37.1 & 49.4 & 59.3 & 68.0 & 74.7 & 80.9 & 86.8 & 92.6 & 95.8 \\
\hline ESF-HD-164-TEMP19-RTD-11 & 5.00 & 22.74 & 0.05 & 5.00 & 28.2 & 38.6 & 51.0 & 61.1 & 69.9 & 76.8 & 83.1 & 88.7 & 93.3 & 96.2 \\
\hline ESF-HD-164-TEMP19-RTD-12 & 5.31 & 22.74 & 0.06 & 5.31 & 27.8 & 39.3 & 52.1 & 62.3 & 71.2 & 78.3 & 84.5 & 89.9 & 94.0 & 96.7 \\
\hline ESF-HD-164-TEMP19-RTD-13 & 5.62 & 22.74 & 0.06 & 5.62 & 27.5 & 40.3 & 53.6 & 63.8 & 72.8 & 80.0 & 86.1 & 91.4 & 95.2 & 97.4 \\
\hline ESF-HD-164-TEMP19-RTD-14 & 5.93 & 22.75 & 0.06 & 5.93 & 27.3 & 40.5 & 54.2 & 64.5 & 73.6 & 80.8 & 86.9 & 92.2 & 95.9 & 97.5 \\
\hline ESF-HD-164-TEMP19-RTD-15 & 6.23 & 22.75 & 0.07 & 6.23 & 26.9 & 40.5 & 54.7 & 65.1 & 74.4 & 81.5 & 87.5 & 93.0 & 96.2 & 97.5 \\
\hline ESF-HD-164-TEMP19-RTD-16 & 6.54 & 22.75 & 0.07 & 6.54 & 26.5 & 40.5 & 55.0 & 65.5 & 74.8 & 82.0 & 88.1 & 93.9 & 96.5 & 97.5 \\
\hline ESF-HD-164-TEMP19-RTD-17 & 6.85 & 22.75 & 0.08 & 6.85 & 26.2 & 40.3 & 55.1 & 65.8 & 75.2 & 82.4 & 88.4 & 94.4 & 96.5 & 97.5 \\
\hline ESF-HD-164-TEMP19-RTD-18 & 7.16 & 22.76 & 0.08 & 7.16 & 25.9 & 39.8 & 54.8 & 65.7 & 75.1 & 82.2 & 88.3 & 94.1 & 96.5 & 97.8 \\
\hline ESF-HD-164-TEMP19-RTD-19 & 7.47 & 22.76 & 0.09 & 7.47 & 25.9 & 39.6 & 54.6 & 65.6 & 74.9 & 82.0 & 87.9 & 93.3 & 96.3 & 98.0 \\
\hline ESF-HD-164-TEMP19-RTD-20 & 7.78 & 22.76 & 0.09 & 7.78 & 25.6 & 38.9 & 54.0 & 65.2 & 74.3 & 81.4 & 87.2 & 92.6 & 96.0 & 98.1 \\
\hline ESF-HD-164-TEMP19-RTD-21 & 8.09 & 22.76 & 0.09 & 8.09 & 25.4 & 38.5 & 53.6 & 64.8 & 73.9 & 80.7 & 86.5 & 91.9 & 95.8 & 98.1 \\
\hline ESF-HD-164-TEMP19-RTD-22 & 8.40 & 22.76 & 0.10 & 8.40 & 25.0 & 37.6 & 53.0 & 64.1 & 73.2 & 80.0 & 85.7 & 91.2 & 95.1 & 97.7 \\
\hline ESF-HD-164-TEMP19-RTD-23 & 8.70 & 22.77 & 0.10 & 8.70 & 25.1 & 37.3 & 52.7 & 63.8 & 72.7 & 79.5 & 85.1 & 90.7 & 94.8 & 97.3 \\
\hline ESF-HD-164-TEMP19-RTD-24 & 9.01 & 22.77 & 0.11 & 9.01 & 24.8 & 36.8 & 52.5 & 63.6 & 72.4 & 79.0 & 84.4 & 90.2 & 94.2 & 96.7 \\
\hline ESF-HD-164-TEMP19-RTD-25 & 9.32 & 22.77 & 0.11 & 9.32 & 24.7 & 36.7 & 52.8 & 63.9 & 72.6 & 79.2 & 84.5 & 90.4 & 94.3 & 96.6 \\
\hline ESF-HD-164-TEMP19-RTD-26 & 9.63 & 22.77 & 0.12 & 9.63 & 24.6 & 37.3 & 53.3 & 64.5 & 73.1 & 79.7 & 85.0 & 91.0 & 94.4 & 95.9 \\
\hline ESF-HD-164-TEMP19-RTD-27 & 9.94 & 22.78 & 0.12 & 9.94 & 24.4 & 38.0 & 54.4 & 65.7 & 74.4 & 81.1 & 86.5 & 92.7 & 95.0 & 96.1 \\
\hline ESF-HD-164-TEMP19-RTD-28 & 10.25 & 22.78 & 0.13 & 10.25 & 24.3 & 38.6 & 55.0 & 66.3 & 75.4 & 82.4 & 88.0 & 93.7 & 96.1 & 97.1 \\
\hline ESF-HD-164-TEMP19-RTD-29 & 10.56 & 22.78 & 0.13 & 10.56 & 24.2 & 39.1 & 55.6 & 66.9 & 76.4 & 83.5 & 88.9 & 93.9 & 96.5 & 97.9 \\
\hline ESF-HD-164-TEMP19-RTD-30 & 10.87 & 22.78 & 0.13 & 10.87 & 24.2 & 39.0 & 55.5 & 66.8 & 76.5 & 83.6 & 88.9 & 93.7 & 96.4 & 97.8 \\
\hline ESF-HD-164-TEMP19-RTD-31 & 11.17 & 22.78 & 0.14 & 11.17 & 24.2 & 39.2 & 55.8 & 67.1 & 76.9 & 83.9 & 89.3 & 94.0 & 96.4 & 97.8 \\
\hline ESF-HD-164-TEMP19-RTD-32 & 11.48 & 22.79 & 0.14 & 11.48 & 24.0 & 39.2 & 55.6 & 67.0 & 76.6 & 83.6 & 89.1 & 93.8 & 96.0 & 97.7 \\
\hline ESF-HD-164-TEMP19-RTD-33 & 11.79 & 22.79 & 0.15 & 11.79 & 24.1 & 39.5 & 55.7 & 66.9 & 76.3 & 83.2 & 88.6 & 93.4 & 95.7 & 97.4 \\
\hline ESF-HD-164-TEMP19-RTD-34 & 12.10 & 22.79 & 0.15 & 12.10 & 24.0 & 39.3 & 55.4 & 66.4 & 75.5 & 82.2 & 87.5 & 92.3 & 95.2 & 97.0 \\
\hline ESF-HD-164-TEMP19-RTD-35 & 12.41 & 22.79 & 0.16 & 12.41 & 24.0 & 39.1 & 55.0 & 65.6 & 74.4 & 80.8 & 86.1 & 90.9 & 94.2 & 96.1 \\
\hline ESF-HD-164-TEMP19-RTD-36 & 12.72 & 22.80 & 0.16 & 12.72 & 24.0 & 38.9 & 54.1 & 64.2 & 72.7 & 78.9 & 84.1 & 88.8 & 92.2 & 94.4 \\
\hline ESF-HD-164-TEMP19-RTD-37 & 13.03 & 22.80 & 0.16 & 13.03 & 23.7 & 38.2 & 52.9 & 62.6 & 70.5 & 76.4 & 81.5 & 86.2 & 89.7 & 92.1 \\
\hline ESF-HD-164-TEMP19-RTD-38 & 13.33 & 22.80 & 0.17 & 13.33 & 23.9 & 37.4 & 51.0 & 59.9 & 67.6 & 73.3 & 78.0 & 82.6 & 86.1 & 88.6 \\
\hline ESF-HD-164-TEMP19-RTD-39 & 13.64 & 22.80 & 0.17 & 13.64 & 23.7 & 36.0 & 48.7 & 57.1 & 64.2 & 69.6 & 74.1 & 78.5 & 82.1 & 84.6 \\
\hline ESF-HD-164-TEMP19-RTD-40 & 13.95 & 22.81 & 0.18 & 13.95 & 23.8 & 34.1 & 45.5 & 53.2 & 59.8 & 64.8 & 69.1 & 73.3 & 76.8 & 79.4 \\
\hline ESF-HD-164-TEMP19-RTD-41 & 14.26 & 22.81 & 0.18 & 14.26 & 23.8 & 32.0 & 41.9 & 48.9 & 54.9 & 59.6 & 63.6 & 67.7 & 71.1 & 73.6 \\
\hline ESF-HD-164-TEMP19-RTD-42 & 14.57 & 22.81 & 0.19 & 14.57 & 23.8 & 29.3 & 37.6 & 43.9 & 49.2 & 53.5 & 57.2 & 60.9 & 64.1 & 66.5 \\
\hline ESF-HD-164-TEMP19-RTD-43 & 14.88 & 22.81 & 0.19 & 14.88 & 23.6 & 27.4 & 34.1 & 39.6 & 44.3 & 48.2 & 51.7 & 55.1 & 58.0 & 60.3 \\
\hline ESF-HD-164-TEMP19-RTD-44 & 15.19 & 22.81 & 0.19 & 15.19 & 23.7 & 26.1 & 31.4 & 36.0 & 40.3 & 43.8 & 46.9 & 50.1 & 52.8 & 54.9 \\
\hline
\end{tabular}


First Quarter Report of Temperature Measurements During the Heating Phase of the Drift-Scale Test

\begin{tabular}{|c|c|c|c|c|c|c|c|c|c|c|c|c|c|c|}
\hline \multirow{5}{*}{\begin{tabular}{|l|} 
\\
ESF-HD-164-TEMP19-RTD-45 \\
\end{tabular}} & \multicolumn{14}{|c|}{ Temperature Data Obtained from the DST } \\
\hline & \multicolumn{14}{|c|}{ Days after startup } \\
\hline & \multicolumn{4}{|c|}{ As Built Locations (m) } & 0 & 7 & 17 & 27 & 38 & 48 & 58 & 69 & 79 & 87 \\
\hline & $\mathbf{x}$ & $\mathbf{y}$ & $\mathbf{z}$ & D & \multicolumn{10}{|c|}{$\left({ }^{\circ} \mathrm{C}\right)$} \\
\hline & 15.50 & 22.82 & 0.20 & 15.50 & 23.7 & 25.0 & 29.1 & 33.0 & 36.7 & 39.9 & 42.7 & 45.7 & 48.2 & 50.1 \\
\hline ESF-HD-164-TEMP19-RTD-46 & 15.80 & 22.82 & 0.20 & 15.80 & 23.7 & 24.4 & 27.3 & 30.4 & 33.7 & 36.5 & 39.1 & 41.7 & 44.1 & 45.9 \\
\hline ESF-HD-164-TEMP19-RTD-47 & 16.11 & 22.82 & 0.21 & 16.11 & 23.6 & 23.9 & 26.1 & 28.7 & 31.5 & 33.9 & 36.3 & 38.8 & 40.9 & 42.6 \\
\hline ESF-HD-164-TEMP19-RTD-48 & 16.42 & 22.82 & 0.21 & 16.42 & 23.4 & 23.6 & 25.2 & 27.3 & 29.6 & 31.8 & 33.9 & 36.1 & 38.1 & 39.6 \\
\hline ESF-HD-164-TEMP19-RTD-49 & 16.73 & 22.83 & 0.22 & 16.73 & 23.6 & 23.6 & 24.7 & 26.3 & 28.3 & 30.1 & 32.0 & 34.0 & 35.8 & 37.2 \\
\hline ESF-HD-164-TEMP19-RTD-50 & 17.04 & 22.83 & 0.22 & 17.04 & 23.4 & 23.5 & 24.3 & 25.5 & 27.1 & 28.7 & 30.3 & 32.1 & 33.7 & 35.0 \\
\hline ESF-HD-164-TEMP19-RTD-51 & 17.35 & 22.83 & 0.23 & 17.35 & 23.6 & 23.6 & 24.0 & 24.9 & 26.3 & 27.6 & 29.1 & 30.7 & 32.1 & 33.3 \\
\hline ESF-HD-164-TEMP19-RTD-52 & 17.66 & 22.83 & 0.23 & 17.66 & 23.6 & 23.6 & 23.8 & 24.5 & 25.6 & 26.7 & 27.9 & 29.3 & 30.6 & 31.7 \\
\hline ESF-HD-164-TEMP19-RTD-53 & 17.96 & 22.83 & 0.23 & 17.96 & 23.6 & 23.6 & 23.8 & 24.3 & 25.1 & 26.1 & 27.1 & 28.3 & 29.5 & 30.4 \\
\hline ESF-HD-164-TEMP19-RTD-54 & 18.27 & 22.84 & 0.24 & 18.27 & 23.5 & 23.6 & 23.7 & 24.0 & 24.7 & 25.4 & 26.3 & 27.4 & 28.4 & 29.2 \\
\hline ESF-HD-164-TEMP19-RTD-55 & 18.58 & 22.84 & 0.24 & 18.58 & 23.5 & 23.5 & 23.6 & 23.9 & 24.4 & 25.0 & 25.8 & 26.7 & 27.5 & 28.3 \\
\hline ESF-HD-164-TEMP19-RTD-56 & 18.89 & 22.84 & 0.25 & 18.89 & 23.6 & 23.6 & 23.6 & 23.8 & 24.2 & 24.7 & 25.3 & 26.1 & 26.8 & 27.5 \\
\hline ESF-HD-164-TEMP19-RTD-57 & 19.20 & 22.84 & 0.25 & 19.20 & 23.5 & 23.5 & 23.6 & 23.7 & 24.0 & 24.4 & 24.9 & 25.6 & 26.2 & 26.8 \\
\hline ESF-HD-164-TEMP19-RTD-58 & 19.51 & 22.85 & 0.26 & 19.51 & 23.6 & 23.6 & 23.6 & 23.7 & 23.9 & 24.2 & 24.6 & 25.2 & 25.7 & 26.2 \\
\hline ESF-HD-164-TEMP19-RTD-59 & 19.82 & 22.85 & 0.26 & 19.82 & 23.4 & 23.5 & 23.6 & 23.6 & 23.8 & 24.0 & 24.3 & 24.8 & 25.3 & 25.7 \\
\hline ESF-HD-164-TEMP19-RTD-60 & 20.13 & 22.85 & 0.26 & 20.13 & 23.5 & 23.5 & 23.5 & 23.6 & 23.7 & 23.9 & 24.1 & 24.5 & 24.9 & 25.3 \\
\hline ESF-HD-164-TEMP19-RTD-61 & 20.43 & 22.85 & 0.27 & 20.43 & 23.5 & 23.5 & 23.5 & 23.6 & 23.7 & 23.8 & 24.0 & 24.3 & 24.7 & 25.0 \\
\hline & 20.74 & 22.86 & 0.27 & 20.74 & 23.6 & & & & 23.7 & & & & 24.5 & 24.8 \\
\hline & 21.05 & 22.86 & 0.28 & 21.05 & 23.4 & 23.4 & & & 23.6 & 23.7 & 23.8 & 24.0 & 24.3 & 24.5 \\
\hline ESF-HD-164-TEMP19-RTD-64 & 21.36 & 22.86 & 0.28 & 21.36 & 23.3 & 23.4 & & 23.6 & 23.6 & 23.6 & 23.7 & 23.9 & 24.1 & 24.3 \\
\hline ESF-HD-164-TEMP19-RTD-65 & 21.67 & 22.86 & 0.29 & 21.67 & 23.6 & 23.6 & 23.6 & 23.6 & 23.6 & 23.6 & 23.7 & 23.8 & 24.0 & 24.2 \\
\hline ESF-HD-164-TEMP19-RTD-66 & 21.98 & 22.86 & 0.29 & 21.98 & 21.6 & 22.1 & 22.9 & 23.0 & 23.0 & 23.0 & 23.1 & 23.2 & 23.3 & 23.5 \\
\hline ESF-HD-164-TEMP19-RTD-67 & 22.29 & 22.87 & 0.29 & 22.29 & 23.5 & 23.5 & 23.6 & 23.6 & 23.6 & 23.6 & 23.6 & 23.7 & 23.8 & 24.0 \\
\hline \multicolumn{15}{|l|}{ BH 165 Gage ID } \\
\hline ESF-HD-165-TEMP20-RTD-1 & 1.68 & 22.85 & 1.70 & 2.39 & 29.8 & 61.1 & 71.1 & 78.0 & 84.4 & 89.2 & 93.6 & 97.6 & 101.8 & 103.8 \\
\hline ESF-HD-165-TEMP20-RTD-2 & 1.89 & 22.84 & 1.91 & 2.69 & 29.9 & 54.1 & 64.5 & 71.6 & 78.0 & 82.8 & 87.2 & 91.6 & 95.5 & 97.8 \\
\hline ESF-HD-165-TEMP20-RTD-3 & 2.11 & 22.84 & 2.13 & 3.00 & 29.7 & 44.4 & 55.0 & 62.1 & 68.6 & 73.7 & 78.3 & 82.7 & 86.5 & 89.1 \\
\hline ESF-HD-165-TEMP20-RTD-4 & 2.32 & 22.84 & 2.34 & 3.30 & 29.7 & 38.8 & 48.1 & 55.0 & 61.3 & 66.4 & 71.0 & 75.6 & 79.3 & 82.1 \\
\hline ESF-HD-165-TEMP20-RTD-5 & 2.53 & 22.84 & 2.55 & 3.59 & 29.5 & 34.8 & 43.1 & 49.6 & 55.7 & 60.7 & 65.3 & 69.8 & 73.6 & 76.5 \\
\hline ESF-HD-165-TEMP20-RTD-6 & 2.75 & 22.84 & 2.77 & 3.90 & 29.3 & 32.2 & 39.1 & 45.1 & 50.9 & 55.8 & 60.2 & 64.7 & 68.5 & 71.4 \\
\hline ESF-HD-165-TEMP20-RTD-7 & 2.96 & 22.84 & 2.98 & 4.20 & 29.1 & 30.6 & 36.2 & 41.5 & 46.9 & 51.5 & 55.8 & 60.2 & 64.0 & 66.8 \\
\hline ESF-HD-165-TEMP20-RTD-8 & 3.17 & 22.84 & 3.19 & 4.50 & 28.8 & 29.4 & 33.6 & 38.3 & 43.2 & 47.6 & 51.7 & 56.0 & 59.6 & 62.4 \\
\hline ESF-HD-165-TEMP20-RTD-9 & 3.38 & 22.84 & 3.40 & 4.79 & 28.4 & 28.7 & 31.8 & 35.8 & 40.3 & 44.4 & 48.3 & 52.4 & 55.9 & 58.7 \\
\hline ESF-HD-165-TEMP20-RTD-10 & 3.60 & 22.83 & 3.62 & 5.11 & 28.1 & 28.2 & 30.3 & 33.6 & 37.6 & 41.3 & 45.0 & 48.9 & 52.3 & 55.0 \\
\hline ESF-HD-165-TEMP20-RTD-11 & 3.81 & 22.83 & 3.83 & 5.40 & 27.7 & 27.7 & 29.2 & 31.9 & 35.4 & 38.8 & 42.2 & 45.9 & 49.1 & 51.7 \\
\hline ESF-HD-165-TEMP20-RTD-12 & 4.02 & 22.83 & 4.04 & 5.70 & 27.0 & 26.8 & 28.4 & 30.5 & 33.4 & 36.2 & 39.5 & 43.1 & 46.1 & 48.5 \\
\hline ESF-HD-165-TEMP20-RTD-13 & 4.24 & 22.83 & 4.25 & 6.00 & 27.1 & 27.0 & 27.7 & 29.4 & 32.0 & 34.7 & 37.6 & 40.8 & 43.8 & 46.1 \\
\hline
\end{tabular}




\begin{tabular}{|c|c|c|c|c|c|c|c|c|c|c|c|c|c|c|}
\hline & \multicolumn{14}{|c|}{ Temperature Data Obtained from the DST } \\
\hline & \multicolumn{14}{|c|}{ Days after startup } \\
\hline & \multicolumn{4}{|c|}{ As Built Locations (m) } & 0 & 7 & 17 & 27 & 38 & 48 & 58 & 69 & 79 & 87 \\
\hline & $\mathbf{x}$ & $\mathbf{y}$ & $\mathbf{z}$ & D & \multicolumn{10}{|c|}{$\left({ }^{\circ} \mathbf{C}\right)$} \\
\hline ESF-HD-165-TEMP20-RTD-14 & 4.45 & 22.83 & 4.47 & 6.31 & 26.9 & 26.8 & 27.2 & 28.5 & 30.7 & 33.1 & 35.7 & 38.7 & 41.4 & 43.6 \\
\hline ESF-HD-165-TEMP20-RTD-15 & 4.66 & 22.83 & 4.68 & 6.60 & 26.4 & 26.5 & 26.8 & 27.8 & 29.6 & 31.7 & 34.1 & 36.9 & 39.4 & 41.5 \\
\hline ESF-HD-165-TEMP20-RTD-16 & 4.87 & 22.83 & 4.89 & 6.90 & 26.1 & 26.2 & 26.4 & 27.2 & 28.7 & 30.5 & 32.6 & 35.1 & 37.5 & 39.5 \\
\hline ESF-HD-165-TEMP20-RTD-17 & 5.09 & 22.82 & 5.11 & 7.21 & 25.9 & 26.0 & 26.2 & 26.7 & 28.0 & 29.6 & 31.4 & 33.8 & 36.0 & 37.8 \\
\hline ESF-HD-165-TEMP20-RTD-18 & 5.30 & 22.82 & 5.32 & 7.51 & 25.7 & 25.8 & 25.9 & 26.3 & 27.3 & 28.7 & 30.3 & 32.4 & 34.4 & 36.1 \\
\hline ESF-HD-165-TEMP20-RTD-19 & 5.51 & 22.82 & 5.53 & 7.81 & 25.5 & 25.2 & 25.5 & 25.9 & 26.7 & 27.8 & 29.3 & 31.2 & 33.1 & 34.7 \\
\hline ESF-HD-165-TEMP20-RTD-20 & 5.72 & 22.82 & 5.74 & 8.10 & 25.2 & 25.3 & 25.4 & 25.7 & 26.3 & 27.3 & 28.5 & 30.2 & 31.9 & 33.3 \\
\hline ESF-HD-165-TEMP20-RTD-21 & 5.94 & 22.82 & 5.96 & 8.41 & 24.7 & 24.9 & 25.2 & 25.5 & 25.9 & 26.7 & 27.8 & 29.3 & 30.9 & 32.2 \\
\hline ESF-HD-165-TEMP20-RTD-22 & 6.15 & 22.82 & 6.17 & 8.71 & 24.6 & 24.6 & 25.1 & 25.3 & 25.6 & 26.3 & 27.2 & 28.6 & 29.5 & 31.1 \\
\hline ESF-HD-165-TEMP20-RTD-23 & 6.36 & 22.82 & 6.38 & 9.01 & 24.8 & 24.8 & 25.0 & 25.1 & 25.4 & 26.0 & 26.8 & 28.0 & 29.2 & 30.4 \\
\hline ESF-HD-165-TEMP20-RTD-24 & 6.58 & 22.81 & 6.59 & 9.31 & 24.5 & 24.7 & 24.8 & 24.9 & 25.2 & 25.7 & 26.4 & 27.4 & 28.5 & 29.5 \\
\hline ESF-HD-165-TEMP20-RTD-25 & 6.79 & 22.81 & 6.81 & 9.62 & 24.5 & 24.5 & 24.7 & 24.8 & 25.0 & 25.3 & 25.9 & 26.8 & 27.8 & 28.7 \\
\hline ESF-HD-165-TEMP20-RTD-26 & 7.00 & 22.81 & 7.02 & 9.91 & 24.3 & 24.4 & 24.6 & 24.7 & 24.8 & 25.1 & 25.6 & 26.4 & 27.3 & 28.1 \\
\hline ESF-HD-165-TEMP20-RTD-27 & 7.21 & 22.81 & 7.23 & 10.21 & 24.3 & 24.4 & 24.5 & 24.5 & 24.7 & 24.9 & 25.4 & 26.0 & 26.8 & 27.5 \\
\hline ESF-HD-165-TEMP20-RTD-28 & 7.43 & 22.81 & 7.45 & 10.52 & 24.1 & 24.2 & 24.3 & 24.4 & 24.5 & 24.7 & 25.1 & 25.7 & 26.3 & 27.0 \\
\hline ESF-HD-165-TEMP20-RTD-29 & 7.64 & 22.81 & 7.66 & 10.82 & 24.1 & 24.1 & 24.2 & 24.3 & 24.4 & 24.6 & 24.9 & 25.4 & 26.0 & 26.6 \\
\hline ESF-HD-165-TEMP20-RTD-30 & 7.85 & 22.81 & 7.87 & 11.12 & 23.9 & 24.0 & 24.1 & 24.2 & 24.3 & 24.4 & 24.7 & 25.1 & 25.6 & 26.1 \\
\hline ESF-HD-165-TEMP20-RTD-31 & 8.07 & 22.81 & 8.08 & 11.42 & 24.0 & 24.0 & 24.1 & 24.1 & 24.2 & 24.3 & 24.5 & 24.9 & 25.4 & 25.8 \\
\hline ESF-HD-165-TEMP20-RTD-32 & 8.28 & 22.80 & 8.30 & 11.72 & 23.6 & 23.7 & 24.0 & 24.0 & 24.1 & 23.9 & 24.0 & 24.6 & 24.9 & 25.4 \\
\hline ESF-HD-165-TEMP20-RTD-33 & 8.49 & 22.80 & 8.51 & 12.02 & 23.8 & 23.9 & 23.9 & 24.0 & 24.0 & 24.1 & 24.3 & 24.5 & 24.9 & 25.2 \\
\hline ESF-HD-165-TEMP20-RTD-34 & 8.70 & 22.80 & 8.72 & 12.32 & 23.7 & 23.8 & 23.8 & 23.9 & 23.9 & 24.0 & 24.1 & 24.4 & 24.6 & 24.9 \\
\hline ESF-HD-165-TEMP20-RTD-35 & 8.92 & 22.80 & 8.93 & 12.62 & 23.7 & 23.8 & 23.8 & 23.8 & 23.9 & 23.9 & 24.0 & 24.2 & 24.5 & 24.7 \\
\hline ESF-HD-165-TEMP20-RTD-36 & 9.13 & 22.80 & 9.15 & 12.93 & 23.6 & 23.6 & 23.7 & 23.8 & 23.8 & 23.8 & 23.9 & 24.1 & 24.3 & 24.5 \\
\hline ESF-HD-165-TEMP20-RTD-37 & 9.34 & 22.80 & 9.36 & 13.22 & 23.7 & 23.7 & 23.7 & 23.8 & 23.8 & 23.8 & 23.9 & 24.0 & 24.2 & 24.4 \\
\hline ESF-HD-165-TEMP20-RTD-38 & 9.55 & 22.80 & 9.57 & 13.52 & 23.5 & 23.5 & 23.6 & 23.7 & 23.7 & 23.7 & 23.8 & 23.9 & 24.0 & 24.2 \\
\hline ESF-HD-165-TEMP20-RTD-39 & 9.77 & 22.79 & 9.79 & 13.83 & 23.6 & 23.5 & 23.6 & 23.6 & 23.7 & 23.7 & 23.7 & 23.8 & 24.0 & 24.1 \\
\hline ESF-HD-165-TEMP20-RTD-40 & 9.98 & 22.79 & 10.00 & 14.13 & 23.6 & 23.6 & 23.6 & 23.6 & 23.7 & 23.7 & 23.7 & 23.8 & 23.9 & 24.0 \\
\hline ESF-HD-165-TEMP20-RTD-41 & 10.19 & 22.79 & 10.21 & 14.43 & 23.5 & 23.6 & 23.6 & 23.6 & 23.6 & 23.7 & 23.7 & 23.8 & 23.8 & 23.9 \\
\hline ESF-HD-165-TEMP20-RTD-42 & 10.41 & 22.79 & 10.42 & 14.73 & 23.6 & 23.6 & 23.7 & 23.7 & 23.7 & 23.7 & 23.7 & 23.8 & 23.9 & 24.0 \\
\hline ESF-HD-165-TEMP20-RTD-43 & 10.62 & 22.79 & 10.64 & 15.03 & 23.5 & 23.5 & 23.5 & 23.6 & 23.6 & 23.6 & 23.6 & 23.7 & 23.7 & 23.8 \\
\hline ESF-HD-165-TEMP20-RTD-44 & 10.83 & 22.79 & 10.85 & 15.33 & 23.4 & 23.4 & 23.5 & 23.5 & 23.5 & 23.5 & 23.6 & 23.6 & 23.7 & 23.7 \\
\hline ESF-HD-165-TEMP20-RTD-45 & 11.04 & 22.79 & 11.06 & 15.63 & 23.5 & 23.5 & 23.5 & 23.5 & 23.5 & 23.5 & 23.6 & 23.6 & 23.6 & 23.7 \\
\hline ESF-HD-165-TEMP20-RTD-46 & 11.26 & 22.78 & 11.27 & 15.93 & 23.3 & 23.3 & 23.4 & 23.5 & 23.5 & 23.5 & 23.5 & 23.5 & 23.6 & 23.6 \\
\hline ESF-HD-165-TEMP20-RTD-47 & 11.47 & 22.78 & 11.49 & 16.24 & 23.5 & 23.5 & 23.5 & 23.5 & 23.5 & 23.5 & 23.5 & 23.5 & 23.6 & 23.6 \\
\hline ESF-HD-165-TEMP20-RTD-48 & 11.68 & 22.78 & 11.70 & 16.53 & 23.3 & 23.3 & 23.4 & 23.4 & 23.4 & 23.5 & 23.5 & 23.5 & 23.5 & 23.5 \\
\hline ESF-HD-165-TEMP20-RTD-49 & 11.90 & 22.78 & 11.91 & 16.84 & 23.4 & 23.4 & 23.4 & 23.5 & 23.5 & 23.5 & 23.5 & 23.5 & 23.5 & 23.5 \\
\hline ESF-HD-165-TEMP20-RTD-50 & 12.11 & 22.78 & 12.13 & 17.14 & 23.4 & 23.4 & 23.4 & 23.4 & 23.4 & 23.4 & 23.5 & 23.5 & 23.5 & 23.5 \\
\hline
\end{tabular}


First Quarter Report of Temperature Measurements During the Heating Phase of the Drift-Scale Test

\begin{tabular}{|c|c|c|c|c|c|c|c|c|c|c|c|c|c|c|}
\hline \multirow{5}{*}{\begin{tabular}{|l|} 
\\
ESF-HD-165-TEMP20-RTD-51 \\
\end{tabular}} & \multicolumn{14}{|c|}{ Temperature Data Obtained from the DST } \\
\hline & \multicolumn{14}{|c|}{ Days after startup } \\
\hline & \multicolumn{4}{|c|}{ As Built Locations (m) } & 0 & 7 & 17 & 27 & 38 & 48 & 58 & 69 & 79 & 87 \\
\hline & \multirow{2}{*}{\begin{tabular}{c|}
$\mathbf{x}$ \\
12.32 \\
\end{tabular}} & \multirow{2}{*}{\begin{tabular}{|c|}
$\mathbf{y}$ \\
22.78 \\
\end{tabular}} & \multirow{2}{*}{\begin{tabular}{c|}
$\mathbf{z}$ \\
12.34 \\
\end{tabular}} & \multirow{2}{*}{$\begin{array}{c}\text { D } \\
17.44 \\
\end{array}$} & \multicolumn{10}{|c|}{$\left({ }^{\circ} \mathbf{C}\right)$} \\
\hline & & & & & 23.3 & 23.3 & 23.4 & 23.4 & 23.4 & 23.4 & 23.4 & 23.5 & 23.5 & 23.5 \\
\hline ESF-HD-165-TEMP20-RTD-52 & 12.53 & 22.78 & 12.55 & 17.73 & 23.4 & 23.4 & 23.4 & 23.4 & 23.4 & 23.4 & 23.4 & 23.5 & 23.5 & 23.5 \\
\hline ESF-HD-165-TEMP20-RTD-53 & 12.75 & 22.78 & 12.76 & 18.04 & 23.4 & 23.4 & 23.4 & 23.4 & 23.4 & 23.4 & 23.4 & 23.4 & 23.5 & 23.5 \\
\hline ESF-HD-165-TEMP20-RTD-54 & 12.96 & 22.77 & 12.98 & 18.34 & 23.3 & 23.3 & 23.4 & 23.4 & 23.4 & 23.4 & 23.4 & 23.4 & 23.4 & 23.4 \\
\hline ESF-HD-165-TEMP20-RTD-55 & 13.17 & 22.77 & 13.19 & 18.64 & 23.2 & 23.3 & 23.4 & 23.4 & 23.4 & 23.4 & 23.4 & 23.4 & 23.4 & 23.4 \\
\hline ESF-HD-165-TEMP20-RTD-56 & 13.38 & 22.77 & 13.40 & 18.94 & 23.2 & 23.2 & 23.3 & 23.3 & 23.3 & 23.3 & 23.3 & 23.3 & 23.3 & 23.3 \\
\hline ESF-HD-165-TEMP20-RTD-57 & 13.60 & 22.77 & 13.61 & 19.24 & 23.3 & 23.3 & 23.3 & 23.4 & 23.4 & 23.4 & 23.4 & 23.4 & 23.4 & 23.4 \\
\hline ESF-HD-165-TEMP20-RTD-58 & 13.81 & 22.77 & 13.83 & 19.54 & 23.2 & 23.2 & 23.3 & 23.3 & 23.3 & 23.3 & 23.3 & 23.4 & 23.3 & 23.3 \\
\hline ESF-HD-165-TEMP20-RTD-59 & 14.02 & 22.77 & 14.04 & 19.84 & 23.3 & 23.2 & 23.3 & 23.4 & 23.4 & 23.4 & 23.4 & 23.4 & 23.4 & 23.4 \\
\hline ESF-HD-165-TEMP20-RTD-60 & 14.24 & 22.77 & 14.25 & 20.15 & 23.3 & 23.3 & 23.3 & 23.3 & 23.3 & 23.3 & 23.4 & 23.4 & 23.4 & 23.4 \\
\hline ESF-HD-165-TEMP20-RTD-61 & 14.45 & 22.76 & 14.47 & 20.45 & 23.4 & 23.4 & 23.4 & 23.4 & 23.4 & 23.4 & 23.4 & 23.4 & 23.4 & 23.4 \\
\hline ESF-HD-165-TEMP20-RTD-62 & 14.66 & 22.76 & 14.68 & 20.75 & 23.2 & 23.3 & 23.3 & 23.3 & 23.3 & 23.3 & 23.3 & 23.3 & 23.3 & 23.3 \\
\hline ESF-HD-165-TEMP20-RTD-63 & 14.87 & 22.76 & 14.89 & 21.04 & 23.3 & 23.3 & 23.3 & 23.3 & 23.3 & 23.3 & 23.3 & 23.3 & 23.3 & 23.3 \\
\hline ESF-HD-165-TEMP20-RTD-64 & 15.09 & 22.76 & 15.10 & 21.35 & 23.3 & 23.3 & 23.3 & 23.3 & 23.3 & 23.3 & 23.3 & 23.3 & 23.3 & 23.3 \\
\hline ESF-HD-165-TEMP20-RTD-65 & 15.30 & 22.76 & 15.32 & 21.65 & 23.3 & 23.3 & 23.3 & 23.3 & 23.3 & 23.3 & 23.3 & 23.3 & 23.3 & 23.3 \\
\hline ESF-HD-165-TEMP20-RTD-66 & 15.51 & 22.76 & 15.53 & 21.95 & 23.2 & 23.2 & 23.3 & 23.3 & 23.3 & 23.3 & 23.3 & 23.3 & 23.3 & 23.3 \\
\hline ESF-HD-165-TEMP20-RTD-67 & 15.72 & 22.76 & 15.74 & 22.25 & 23.3 & 23.3 & 23.3 & 23.3 & 23.3 & 23.3 & & 23.3 & 23.3 & 23.3 \\
\hline \multicolumn{15}{|l|}{ BH 168 Gage ID } \\
\hline ESF-HD-168-TEMP21-RTD-1 & -0.08 & 31.95 & 3.16 & 3.16 & 30.3 & 46.5 & 56.5 & 63.2 & 69.2 & 74.0 & 78.2 & 82.1 & 85.1 & 87.4 \\
\hline ESF-HD-168-TEMP21-RTD-2 & -0.08 & 31.95 & 3.46 & 3.46 & 30.2 & 41.0 & 50.4 & 56.9 & 62.6 & 67.2 & 71.3 & 75.2 & 78.2 & 80.5 \\
\hline ESF-HD-168-TEMP21-RTD-3 & -0.09 & 31.95 & 3.76 & 3.76 & 30.1 & 36.3 & 44.8 & 51.0 & 56.5 & 61.0 & 65.0 & 68.9 & 72.1 & 74.5 \\
\hline ESF-HD-168-TEMP21-RTD-4 & -0.09 & 31.95 & 4.06 & 4.06 & 29.8 & 33.4 & 40.6 & 46.4 & 51.6 & 55.9 & 59.7 & 63.7 & 66.8 & 69.2 \\
\hline ESF-HD-168-TEMP21-RTD-5 & -0.10 & 31.95 & 4.36 & 4.36 & 29.6 & 31.4 & 37.1 & 42.1 & 46.9 & 50.9 & 54.5 & 58.2 & 61.3 & 63.7 \\
\hline ESF-HD-168-TEMP21-RTD-6 & -0.10 & 31.96 & 4.66 & 4.66 & 29.3 & 30.1 & 34.4 & 38.8 & 43.1 & 46.7 & 50.1 & 53.6 & 56.6 & 58.9 \\
\hline ESF-HD-168-TEMP21-RTD-7 & -0.10 & 31.96 & 4.96 & 4.96 & 28.9 & 29.1 & 32.2 & 35.8 & 39.6 & 42.9 & 46.0 & 49.3 & 52.1 & 54.3 \\
\hline ESF-HD-168-TEMP21-RTD-8 & -0.11 & 31.96 & 5.26 & 5.26 & 28.5 & 28.6 & 30.8 & 33.8 & 37.1 & 40.0 & 42.9 & 45.9 & 48.6 & 50.7 \\
\hline ESF-HD-168-TEMP21-RTD-9 & -0.11 & 31.96 & 5.56 & 5.56 & 28.2 & 28.2 & 29.6 & 32.0 & 34.8 & 37.4 & 40.0 & 42.8 & 45.3 & 47.2 \\
\hline ESF-HD-168-TEMP21-RTD-10 & -0.12 & 31.96 & 5.86 & 5.86 & 27.8 & 27.8 & 28.7 & 30.6 & 33.0 & 35.3 & 37.7 & 40.2 & 42.5 & 44.3 \\
\hline ESF-HD-168-TEMP21-RTD-11 & -0.12 & 31.96 & 6.16 & 6.16 & 27.5 & 27.4 & 28.0 & 29.5 & 31.5 & 33.5 & 35.5 & 37.8 & 39.9 & 41.6 \\
\hline ESF-HD-168-TEMP21-RTD-12 & -0.12 & 31.96 & 6.46 & 6.46 & 27.1 & 27.2 & 27.5 & 28.6 & 30.3 & 32.0 & 33.8 & 35.9 & 37.8 & 39.3 \\
\hline ESF-HD-168-TEMP21-RTD-13 & -0.13 & 31.96 & 6.76 & 6.76 & 26.4 & 26.1 & 27.0 & 27.8 & 28.9 & 30.4 & 32.0 & 34.0 & 35.5 & 37.1 \\
\hline ESF-HD-168-TEMP21-RTD-14 & -0.13 & 31.96 & 7.06 & 7.06 & 26.5 & 26.5 & 26.7 & 27.3 & 28.4 & 29.6 & 30.9 & 32.6 & 34.1 & 35.4 \\
\hline ESF-HD-168-TEMP21-RTD-15 & -0.14 & 31.96 & 7.36 & 7.36 & 26.2 & 26.3 & 26.4 & 26.8 & 27.7 & 28.7 & 29.8 & 31.3 & 32.6 & 33.8 \\
\hline ESF-HD-168-TEMP21-RTD-16 & -0.14 & 31.96 & 7.66 & 7.66 & 25.9 & 26.0 & 26.1 & 26.4 & 27.1 & 27.8 & 28.8 & 30.2 & 31.4 & 32.4 \\
\hline ESF-HD-168-TEMP21-RTD-17 & -0.14 & 31.96 & 7.96 & 7.96 & 25.7 & 25.8 & 25.9 & 26.1 & 26.6 & 27.3 & 28.2 & 29.2 & 30.3 & 31.3 \\
\hline ESF-HD-168-TEMP21-RTD-18 & -0.15 & 31.96 & 8.26 & 8.26 & 25.1 & 25.4 & 25.6 & 25.8 & 26.2 & 26.8 & 27.5 & 28.4 & 29.4 & 30.2 \\
\hline ESF-HD-168-TEMP21-RTD-19 & -0.15 & 31.96 & 8.56 & 8.56 & 24.3 & 24.3 & 25.3 & 25.5 & 25.8 & 26.2 & 26.9 & 27.7 & 28.5 & 29.2 \\
\hline
\end{tabular}




\begin{tabular}{|c|c|c|c|c|c|c|c|c|c|c|c|c|c|c|}
\hline \multirow{5}{*}{ ESF-HD-168-TEMP21-RTD-20 } & \multicolumn{14}{|c|}{ Temperature Data Obtained from the DST } \\
\hline & \multicolumn{14}{|c|}{ Days after startup } \\
\hline & \multicolumn{4}{|c|}{ As Built Locations (m) } & 0 & 7 & 17 & 27 & 38 & 48 & 58 & 69 & 79 & 87 \\
\hline & $\mathbf{x}$ & y & $z$ & D & \multicolumn{10}{|c|}{$\left({ }^{\circ} \mathrm{C}\right)$} \\
\hline & -0.15 & 31.96 & 8.86 & 8.86 & 25.1 & 25.1 & & 25.3 & 25.6 & 25.9 & & 7.1 & 27.8 & 8.5 \\
\hline ESF-HD-168-TEMP21-RTD-21 & -0.16 & 31.96 & & 16 & 9 & 5.0 & 5.1 & 5.2 & .4 & 5.7 & 6.1 & 8.6 & 7.2 & 7.8 \\
\hline ESF-HD-168-TEMP21-F & -0.16 & 31.96 & & 9.46 & 4.7 & 24.8 & 1.9 & 5.0 & 5.1 & .4 & 25.7 & 0.2 & 26.7 & 7.2 \\
\hline ESF-H & -0.17 & 3 & & 9.76 & 6 & .7 & 8 & 4.8 & .0 & 5.2 & 5.5 & 5.9 & 26.3 & 6.7 \\
\hline ESF-HD-168-TEMP21-RTD-24 & -0.17 & 31.96 & 10.06 & 10.06 & 24.5 & 24.5 & 24.6 & 4.7 & .8 & 24.9 & 25.2 & 5.5 & 25.9 & 6.3 \\
\hline ESF-HD-168-TEM & -0.17 & .96 & 10.36 & 10.36 & 24.4 & 24.4 & .5 & 4.5 & .6 & 4.8 & 24.9 & 5.2 & 25.6 & \\
\hline TD-26 & -0.18 & 31.96 & 10.66 & 10.66 & 24.2 & 24.3 & & 4.4 & .5 & & & 5.0 & 25.3 & \\
\hline SF-HD-168-TEMP21-RTD-27 & -0.18 & 1.96 & 10.96 & 10.96 & 24 & 24.2 & & 4.3 & & & & 4.8 & 25.0 & \\
\hline SF-HD-168-TEMP21-RTD-28 & -0.19 & & & 11.26 & 24.1 & 4.1 & & 4.2 & & & & 4.6 & 24.9 & \\
\hline AP21-RTD-29 & -0.19 & & & 11.56 & 24 & & & & & & & & & \\
\hline IP21-RTD-30 & -0.19 & & & 11.86 & & & & & & & & & & \\
\hline-31 & -0.20 & & 2.17 & 12.17 & & & & & & & & & 4.4 & \\
\hline-32 & -0.20 & & 2.47 & 12.47 & 23.8 & 23.8 & & & & & & ..2 & 24.2 & \\
\hline D-33 & -0.21 & & 2.77 & 12.77 & 23.7 & 23.7 & & 0.9 & 3.9 & & & 4.1 & 24.2 & \\
\hline-34 & -0.21 & 1.97 & 3.07 & 13.07 & 23.5 & 23.6 & & 0.0 & 3.8 & 0.0 & & 3.9 & 24.0 & \\
\hline-35 & -0.21 & 1.97 & 3.37 & 13.37 & 23.6 & 2 & & & & & & & 23.9 & \\
\hline-36 & -0.22 & & 3.67 & 13.67 & 23.9 & 2 & & & & & & & 4.2 & \\
\hline & -0.22 & & & 13 & 23.6 & & & & & & & & & \\
\hline & -0.23 & & 4.27 & 14 & 23.5 & & & & & & & & 3.8 & \\
\hline & & & & & & & & & & & & & & \\
\hline & & & & & & & & & & & & & & \\
\hline & & & & & & & & & & & & & & \\
\hline & & & & & & & & & & & & & & \\
\hline & -0.25 & & & & & & & & & & & & & \\
\hline & -0.25 & & & 16.07 & & & & & & & & & & \\
\hline SF-HD-168-TEMP21-RTD-45 & -0.25 & & & & & & & & & & & & & \\
\hline & -0.26 & & & & & & & & & & & & & \\
\hline 1-RTD-47 & -0.26 & & & & & & & & & & & & & \\
\hline rD-48 & -0.26 & & 17.27 & 17.27 & & & & & & & & & & \\
\hline MP21-RTD-49 & -0.27 & 31.97 & 17.57 & 17.57 & & & & & & & & & & \\
\hline MP21-RTD-50 & -0.27 & & 17.87 & 17.87 & & & & & & & & & & \\
\hline SF-HD-168-TEMP21-RTD-51 & -0.28 & & 18.17 & 18.17 & 23.3 & & & & & & & & & \\
\hline & -0.28 & 31.97 & 18.47 & 18.47 & 23.3 & 23.3 & & & & & & & 23.3 & \\
\hline & -0.28 & 31.97 & 18.77 & 18.77 & & & & & & & & & & \\
\hline & -0.29 & & 19.07 & & & & & & & & & & 23.3 & \\
\hline & -0.29 & 31.97 & 19.37 & 19.37 & & 23.2 & & 23.3 & & & & 23.3 & 23.3 & \\
\hline & -0.30 & & 19.67 & $\mid 19.67$ & & & & & 23.3 & & 23.3 & & 23.3 & \\
\hline
\end{tabular}


First Quarter Report of Temperature Measurements During the Heating Phase of the Drift-Scale Test

\begin{tabular}{|c|c|c|c|c|c|c|c|c|c|c|c|c|c|c|}
\hline \multirow{5}{*}{\begin{tabular}{|l|} 
\\
ESF-HD-168-TEMP21-RTD-57 \\
\end{tabular}} & \multicolumn{14}{|c|}{ Temperature Data Obtained from the DST } \\
\hline & \multicolumn{14}{|c|}{ Days after startup } \\
\hline & \multicolumn{4}{|c|}{ As Built Locations (m) } & 0 & 7 & 17 & 27 & 38 & 48 & 58 & 69 & 79 & 87 \\
\hline & \multirow{2}{*}{$\begin{array}{c}\mathbf{x} \\
-0.30 \\
\end{array}$} & \multirow{2}{*}{\begin{tabular}{|c|}
$\mathbf{y}$ \\
31.97 \\
\end{tabular}} & \multirow{2}{*}{$\begin{array}{c}\mathbf{z} \\
19.97 \\
\end{array}$} & \multirow{2}{*}{$\begin{array}{c}\text { D } \\
19.97 \\
\end{array}$} & \multicolumn{10}{|c|}{$\left({ }^{\circ} \mathbf{C}\right)$} \\
\hline & & & & & 23.3 & 23.3 & 23.3 & 23.3 & 23.3 & 23.3 & 23.3 & 23.3 & 23.3 & 23.3 \\
\hline ESF-HD-168-TEMP21-RTD-58 & -0.30 & 31.97 & 20.27 & 20.27 & 23.3 & 23.2 & 23.2 & 23.3 & 23.3 & 23.2 & 23.3 & 23.3 & 23.3 & 23.3 \\
\hline ESF-HD-168-TEMP21-RTD-59 & -0.31 & 31.98 & 20.57 & 20.57 & 23.3 & 23.3 & 23.3 & 23.3 & 23.3 & 23.3 & 23.3 & 23.3 & 23.3 & 23.3 \\
\hline ESF-HD-168-TEMP21-RTD-60 & -0.31 & 31.98 & 20.87 & 20.87 & 23.2 & 23.2 & 23.2 & 23.2 & 23.2 & 23.2 & 23.2 & 23.2 & 23.2 & 23.2 \\
\hline ESF-HD-168-TEMP21-RTD-61 & -0.32 & 31.98 & 21.17 & 21.17 & 23.2 & 23.2 & 23.2 & 23.2 & 23.2 & 23.2 & 23.2 & 23.2 & 23.2 & 23.2 \\
\hline ESF-HD-168-TEMP21-RTD-62 & -0.32 & 31.98 & 21.47 & 21.47 & 23.1 & 23.1 & 23.2 & 23.2 & 23.2 & 23.2 & 23.2 & 23.2 & 23.2 & 23.2 \\
\hline ESF-HD-168-TEMP21-RTD-63 & -0.32 & 31.98 & 21.77 & 21.77 & 23.2 & 23.2 & 23.2 & 23.2 & 23.2 & 23.2 & 23.2 & 23.2 & 23.2 & 23.2 \\
\hline ESF-HD-168-TEMP21-RTD-64 & -0.33 & 31.98 & 22.07 & 22.07 & 23.1 & 23.1 & 23.2 & 23.2 & 23.2 & 23.2 & 23.2 & 23.2 & 23.2 & 23.2 \\
\hline ESF-HD-168-TEMP21-RTD-65 & -0.33 & 31.98 & 22.37 & 22.37 & 23.2 & 23.1 & 23.2 & 23.2 & 23.2 & 23.2 & 23.2 & 23.2 & 23.2 & 23.2 \\
\hline ESF-HD-168-TEMP21-RTD-66 & -0.34 & 31.98 & 22.67 & 22.67 & 23.2 & 23.1 & 23.1 & 23.1 & 23.1 & 23.1 & 23.1 & 23.1 & 23.1 & 23.1 \\
\hline ESF-HD-168-TEMP21-RTD-67 & -0.34 & 31.98 & 22.97 & 22.97 & 23.2 & 23.1 & 23.1 & 23.1 & 23.1 & 23.1 & 23.1 & 23.1 & 23.1 & 23.1 \\
\hline \multicolumn{15}{|l|}{ BH 169 Gage ID } \\
\hline ESF-HD-169-TEMP22-RTD-1 & 0.00 & 32.02 & -2.01 & 2.01 & 30.7 & 39.1 & 48.8 & 55.8 & 62.1 & 67.1 & 71.6 & 76.0 & 79.6 & 82.3 \\
\hline ESF-HD-169-TEMP22-RTD-2 & 0.01 & 32.02 & -2.32 & 2.32 & 30.6 & 35.8 & 40.2 & 49.2 & 51.1 & 57.0 & 61.6 & 69.0 & 69.2 & 75.5 \\
\hline ESF-HD-169-TEMP22-RTD-3 & 0.01 & 32.03 & -2.62 & 2.62 & 30.5 & 33.4 & 40.7 & 46.8 & 52.4 & 57.0 & 61.1 & 65.4 & 68.9 & 71.5 \\
\hline ESF-HD-169-TEMP22-RTD-4 & 0.01 & 32.03 & -2.92 & 2.92 & 30.2 & 31.7 & 37.5 & 42.8 & 48.0 & 52.2 & 56.2 & 60.3 & 63.7 & 66.2 \\
\hline ESF-HD-169-TEMP22-RTD-5 & 0.02 & 32.04 & -3.23 & 3.23 & 22.0 & 22.5 & 27.4 & 33.0 & 38.8 & 43.7 & 52.1 & 58.4 & 60.0 & 60.2 \\
\hline ESF-HD-169-TEMP22-RTD-6 & 0.02 & 32.05 & -3.53 & 3.53 & 29.5 & 29.7 & 32.7 & 36.5 & 40.6 & 44.2 & 47.6 & 51.3 & 54.4 & 56.8 \\
\hline ESF-HD-169-TEMP22-RTD-7 & 0.02 & 32.05 & -3.83 & 3.83 & 29.1 & 29.1 & 31.2 & 34.3 & 37.9 & 41.1 & 44.3 & 47.6 & 50.6 & 52.8 \\
\hline ESF-HD-169-TEMP22-RTD-8 & 0.03 & 32.06 & -4.14 & 4.14 & 28.7 & 28.7 & 29.9 & 32.4 & 35.4 & 38.3 & 41.1 & 44.2 & 47.0 & 49.1 \\
\hline ESF-HD-169-TEMP22-RTD-9 & 0.03 & 32.06 & -4.44 & 4.44 & 28.4 & 28.3 & 29.1 & 31.0 & 33.6 & 36.1 & 38.7 & 41.5 & 44.1 & 46.1 \\
\hline ESF-HD-169-TEMP22-RTD-10 & 0.04 & 32.07 & -4.74 & 4.74 & 28.0 & 27.9 & 28.4 & 29.8 & 31.9 & 34.1 & 36.3 & 38.9 & 41.3 & 43.1 \\
\hline ESF-HD-169-TEMP22-RTD-11 & 0.04 & 32.08 & -5.05 & 5.05 & 24.0 & 24.0 & 24.2 & 25.1 & 26.6 & 28.3 & 30.1 & 32.3 & 34.3 & 36.1 \\
\hline ESF-HD-169-TEMP22-RTD-12 & 0.04 & 32.08 & -5.35 & 5.35 & 27.3 & 27.3 & 27.5 & 28.2 & 29.5 & 31.1 & 32.8 & 34.9 & 36.8 & 38.4 \\
\hline ESF-HD-169-TEMP22-RTD-13 & 0.05 & 32.09 & -5.65 & 5.65 & 27.0 & 27.0 & 27.1 & 27.6 & 28.7 & 30.0 & 31.5 & 33.3 & 35.0 & 36.4 \\
\hline ESF-HD-169-TEMP22-RTD-14 & 0.05 & 32.09 & -5.96 & 5.96 & 26.5 & 26.5 & 26.7 & 27.1 & 27.9 & 29.0 & 30.2 & 31.8 & 33.3 & 34.6 \\
\hline ESF-HD-169-TEMP22-RTD-15 & 0.05 & 32.10 & -6.26 & 6.26 & 25.3 & 25.7 & 25.8 & 26.1 & 26.7 & 27.4 & 28.5 & 29.9 & 31.4 & 32.6 \\
\hline ESF-HD-169-TEMP22-RTD-16 & 0.06 & 32.11 & -6.56 & 6.56 & 26.0 & 26.2 & 26.3 & 26.5 & 26.9 & 27.6 & 28.5 & 29.6 & 30.8 & 31.9 \\
\hline ESF-HD-169-TEMP22-RTD-17 & 0.06 & 32.11 & -6.87 & 6.87 & 25.3 & 25.3 & 26.0 & 26.1 & 26.5 & 27.0 & 27.8 & 28.7 & 29.8 & 30.7 \\
\hline ESF-HD-169-TEMP22-RTD-18 & 0.07 & 32.12 & -7.17 & 7.17 & 25.6 & 25.7 & 25.8 & 25.9 & 26.2 & 26.6 & 27.2 & 28.0 & 28.9 & 29.7 \\
\hline ESF-HD-169-TEMP22-RTD-19 & 0.07 & 32.12 & -7.47 & 7.47 & 25.4 & 25.5 & 25.6 & 25.7 & 25.9 & 26.2 & 26.7 & 27.4 & 28.2 & 28.9 \\
\hline ESF-HD-169-TEMP22-RTD-20 & 0.07 & 32.13 & -7.78 & 7.78 & 25.2 & 25.3 & 25.4 & 25.5 & 25.7 & 25.9 & 26.3 & 26.9 & 27.5 & 28.1 \\
\hline ESF-HD-169-TEMP22-RTD-21 & 0.08 & 32.14 & -8.08 & 8.08 & 25.1 & 25.1 & 25.2 & 25.3 & 25.4 & 25.6 & 26.0 & 26.4 & 27.0 & 27.5 \\
\hline ESF-HD-169-TEMP22-RTD-22 & 0.08 & 32.14 & -8.38 & 8.38 & 24.9 & 25.0 & 25.1 & 25.2 & 25.3 & 25.3 & 25.6 & 26.2 & 26.5 & 27.0 \\
\hline ESF-HD-169-TEMP22-RTD-23 & 0.08 & 32.15 & -8.69 & 8.69 & 24.8 & 24.9 & 25.0 & 25.0 & 25.1 & 25.3 & 25.5 & 25.8 & 26.2 & 26.5 \\
\hline ESF-HD-169-TEMP22-RTD-24 & 0.09 & 32.16 & -8.99 & 8.99 & 24.7 & 24.7 & 24.8 & 24.9 & 25.0 & 25.1 & 25.2 & 25.5 & 25.8 & 26.1 \\
\hline ESF-HD-169-TEMP22-RTD-25 & 0.09 & 32.16 & -9.29 & 9.29 & 24.3 & 24.4 & 24.7 & 24.8 & 24.8 & 24.9 & 25.1 & 25.3 & 25.5 & 25.8 \\
\hline
\end{tabular}




\begin{tabular}{|c|c|c|c|c|c|c|c|c|c|c|c|c|c|c|}
\hline & \multicolumn{14}{|c|}{ Temperature Data Obtained from the DST } \\
\hline & \multicolumn{14}{|c|}{ Days after startup } \\
\hline & \multicolumn{4}{|c|}{ As Built Locations (m) } & 0 & 7 & 17 & 27 & 38 & 48 & 58 & 69 & 79 & 87 \\
\hline & $\mathbf{x}$ & $\mathbf{y}$ & $\mathbf{z}$ & D & \multicolumn{10}{|c|}{$\left({ }^{\circ} \mathbf{C}\right)$} \\
\hline ESF-HD-169-TEMP22-RTD-26 & 0.09 & 32.17 & -9.60 & 9.60 & 24.4 & 24.5 & 24.5 & 24.6 & 24.7 & 24.8 & 24.9 & 25.1 & 25.3 & 25.5 \\
\hline ESF-HD-169-TEMP22-RTD-27 & 0.10 & 32.17 & -9.90 & 9.90 & 24.0 & 23.1 & 24.1 & 24.5 & 24.5 & 24.7 & 24.8 & 24.9 & 25.1 & 25.3 \\
\hline ESF-HD-169-TEMP22-RTD-28 & 0.10 & 32.18 & -10.20 & 10.20 & 24.3 & 24.3 & 24.3 & 24.5 & 24.5 & 24.6 & 24.7 & 24.8 & 24.9 & 25.1 \\
\hline ESF-HD-169-TEMP22-RTD-29 & 0.11 & 32.19 & -10.51 & 10.51 & 24.3 & 24.3 & 24.3 & 24.4 & 24.5 & 24.5 & 24.6 & 24.6 & 24.8 & 25.0 \\
\hline ESF-HD-169-TEMP22-RTD-30 & 0.11 & 32.19 & -10.81 & 10.81 & 24.2 & 24.2 & 24.3 & 24.3 & 24.4 & 24.4 & 24.5 & 24.6 & 24.7 & 24.8 \\
\hline ESF-HD-169-TEMP22-RTD-31 & 0.11 & 32.20 & -11.11 & 11.11 & 24.1 & 24.2 & 24.2 & 24.3 & 24.3 & 24.3 & 24.4 & 24.5 & 24.6 & 24.6 \\
\hline ESF-HD-169-TEMP22-RTD-32 & 0.12 & 32.20 & -11.42 & 11.42 & 24.1 & 24.1 & 24.2 & 24.2 & 24.3 & 24.3 & 24.3 & 24.4 & 24.5 & 24.5 \\
\hline ESF-HD-169-TEMP22-RTD-33 & 0.12 & 32.21 & -11.72 & 11.72 & 24.0 & 24.0 & 24.1 & 24.1 & 24.2 & 24.2 & 24.3 & 24.3 & 24.4 & 24.4 \\
\hline ESF-HD-169-TEMP22-RTD-34 & 0.12 & 32.22 & -12.02 & 12.02 & 24.0 & 24.0 & 24.1 & 24.1 & 24.1 & 24.2 & 24.2 & 24.2 & 24.3 & 24.4 \\
\hline ESF-HD-169-TEMP22-RTD-35 & 0.13 & 32.22 & -12.33 & 12.33 & 24.0 & 24.0 & 24.0 & 24.0 & 24.1 & 24.1 & 24.1 & 24.2 & 24.2 & 24.3 \\
\hline ESF-HD-169-TEMP22-RTD-36 & 0.13 & 32.23 & -12.63 & 12.63 & 24.0 & 24.0 & 24.0 & 24.1 & 24.1 & 24.1 & 24.2 & 24.2 & 24.2 & 24.3 \\
\hline ESF-HD-169-TEMP22-RTD-37 & 0.14 & 32.23 & -12.93 & 12.93 & 23.8 & 23.8 & 24.0 & 24.0 & 24.0 & 24.1 & 24.1 & 24.1 & 24.1 & 24.2 \\
\hline ESF-HD-169-TEMP22-RTD-38 & 0.14 & 32.24 & -13.24 & 13.24 & 23.9 & 23.9 & 24.0 & 24.0 & 24.0 & 24.0 & 24.1 & 24.1 & 24.1 & 24.1 \\
\hline ESF-HD-169-TEMP22-RTD-39 & 0.14 & 32.25 & -13.54 & 13.54 & 23.9 & 23.9 & 24.0 & 24.0 & 24.0 & 24.0 & 24.0 & 24.1 & 24.1 & 24.1 \\
\hline ESF-HD-169-TEMP22-RTD-40 & 0.15 & 32.25 & -13.84 & 13.84 & 23.7 & 23.7 & 23.8 & 23.9 & 23.9 & 23.9 & 23.9 & 24.0 & 24.0 & 24.1 \\
\hline ESF-HD-169-TEMP22-RTD-41 & 0.15 & 32.26 & -14.15 & 14.15 & 23.9 & 23.9 & 23.9 & 23.9 & 23.9 & 23.9 & 23.9 & 24.0 & 24.0 & 24.0 \\
\hline ESF-HD-169-TEMP22-RTD-42 & 0.15 & 32.27 & -14.45 & 14.45 & 23.8 & 23.8 & 23.9 & 23.9 & 24.0 & 24.0 & 24.0 & 24.0 & 24.0 & 24.0 \\
\hline ESF-HD-169-TEMP22-RTD-43 & 0.16 & 32.27 & -14.75 & 14.75 & 23.9 & 23.9 & 23.9 & 23.9 & 23.9 & 23.9 & 23.9 & 24.0 & 24.0 & 24.0 \\
\hline ESF-HD-169-TEMP22-RTD-44 & 0.16 & 32.28 & -15.06 & 15.06 & 23.7 & 23.7 & 23.8 & 23.9 & 23.8 & 23.8 & 23.8 & 23.9 & 23.9 & 23.9 \\
\hline ESF-HD-169-TEMP22-RTD-45 & 0.17 & 32.28 & -15.36 & 15.36 & 23.8 & 23.9 & 23.9 & 23.9 & 23.9 & 23.9 & 23.9 & 23.9 & 23.9 & 24.0 \\
\hline ESF-HD-169-TEMP22-RTD-46 & 0.17 & 32.29 & -15.66 & 15.66 & 23.9 & 23.8 & 23.8 & 23.9 & 23.9 & 23.9 & 23.9 & 23.9 & 23.9 & 23.9 \\
\hline ESF-HD-169-TEMP22-RTD-47 & 0.17 & 32.30 & -15.97 & 15.97 & 23.8 & 23.8 & 23.8 & 23.9 & 23.9 & 23.9 & 23.9 & 23.9 & 23.9 & 23.9 \\
\hline ESF-HD-169-TEMP22-RTD-48 & 0.18 & 32.30 & -16.27 & 16.27 & 23.9 & 23.9 & 23.9 & 23.9 & 23.9 & 23.9 & 23.9 & 23.9 & 23.9 & 23.9 \\
\hline ESF-HD-169-TEMP22-RTD-49 & 0.18 & 32.31 & -16.57 & 16.57 & 23.8 & 23.8 & 23.9 & 23.9 & 23.9 & 23.9 & 23.9 & 23.9 & 23.9 & 23.9 \\
\hline ESF-HD-169-TEMP22-RTD-50 & 0.18 & 32.31 & -16.88 & 16.88 & 23.7 & 23.6 & 23.8 & 23.8 & 23.8 & 23.8 & 23.8 & 23.8 & 23.8 & 23.8 \\
\hline ESF-HD-169-TEMP22-RTD-51 & 0.19 & 32.32 & -17.18 & 17.18 & 23.6 & 23.6 & 23.8 & 23.8 & 23.8 & 23.8 & 23.8 & 23.9 & 23.9 & 23.9 \\
\hline ESF-HD-169-TEMP22-RTD-52 & 0.19 & 32.33 & -17.48 & 17.48 & 23.8 & 23.8 & 23.8 & 23.8 & 23.9 & 23.9 & 23.9 & 23.9 & 23.8 & 23.9 \\
\hline ESF-HD-169-TEMP22-RTD-53 & 0.20 & 32.33 & -17.79 & 17.79 & 24.0 & 23.9 & 23.9 & 23.9 & 24.0 & 24.0 & 24.0 & 24.0 & 24.0 & 24.0 \\
\hline ESF-HD-169-TEMP22-RTD-54 & 0.20 & 32.34 & -18.09 & 18.09 & 23.8 & 23.9 & 23.9 & 23.9 & 23.9 & 23.9 & 23.9 & 23.9 & 23.9 & 23.9 \\
\hline ESF-HD-169-TEMP22-RTD-55 & 0.20 & 32.34 & -18.39 & 18.39 & 23.7 & 23.7 & 23.8 & 23.8 & 23.8 & 23.8 & 23.8 & 23.8 & 23.8 & 23.8 \\
\hline ESF-HD-169-TEMP22-RTD-56 & 0.21 & 32.35 & -18.70 & 18.70 & 23.8 & 23.8 & 23.8 & 23.8 & 23.8 & 23.8 & 23.8 & 23.8 & 23.8 & 23.8 \\
\hline ESF-HD-169-TEMP22-RTD-57 & 0.21 & 32.36 & -19.00 & 19.00 & 23.8 & 23.8 & 23.8 & 23.8 & 23.8 & 23.8 & 23.8 & 23.8 & 23.8 & 23.8 \\
\hline ESF-HD-169-TEMP22-RTD-58 & 0.21 & 32.36 & -19.30 & 19.30 & 23.6 & 23.5 & 23.8 & 23.8 & 23.8 & 23.8 & 23.8 & 23.8 & 23.8 & 23.8 \\
\hline ESF-HD-169-TEMP22-RTD-59 & 0.22 & 32.37 & -19.61 & 19.61 & 23.9 & 23.9 & 23.9 & 23.9 & 23.9 & 23.9 & 23.9 & 23.9 & 23.9 & 23.9 \\
\hline ESF-HD-169-TEMP22-RTD-60 & 0.22 & 32.38 & -19.91 & 19.91 & 23.8 & 23.8 & 23.8 & 23.8 & 23.7 & 23.7 & 23.7 & 23.8 & 23.8 & 23.8 \\
\hline ESF-HD-169-TEMP22-RTD-61 & 0.23 & 32.38 & -20.21 & 20.21 & 23.8 & 23.7 & 23.7 & 23.8 & 23.8 & 23.8 & 23.9 & 23.9 & 23.9 & 23.9 \\
\hline ESF-HD-169-TEMP22-RTD-62 & 0.23 & 32.39 & -20.52 & 20.52 & 23.6 & 23.6 & 23.8 & 23.8 & 23.8 & 23.8 & 23.8 & 23.8 & 23.8 & 23.8 \\
\hline
\end{tabular}


First Quarter Report of Temperature Measurements During the Heating Phase of the Drift-Scale Test

\begin{tabular}{|c|c|c|c|c|c|c|c|c|c|c|c|c|c|c|}
\hline & \multicolumn{14}{|c|}{ Temperature Data Obtained from the DST } \\
\hline & \multicolumn{14}{|c|}{ Days after startup } \\
\hline & \multicolumn{4}{|c|}{ As Built Locations (m) } & 0 & 7 & 17 & 27 & 38 & 48 & 58 & 69 & 79 & 87 \\
\hline & $\mathbf{x}$ & $\mathbf{y}$ & $\mathbf{z}$ & D & \multicolumn{10}{|c|}{$\left({ }^{\circ} \mathbf{C}\right)$} \\
\hline ESF-HD-169-TEMP22-RTD-63 & 0.23 & 32.39 & -20.82 & 20.82 & 23.8 & 23.8 & 23.8 & 23.9 & 23.9 & 23.9 & 23.9 & 23.9 & 23.9 & 23.9 \\
\hline ESF-HD-169-TEMP22-RTD-64 & 0.24 & 32.40 & -21.12 & 21.12 & 23.8 & 23.8 & 23.8 & 23.8 & 23.8 & 23.8 & 23.8 & 23.8 & 23.9 & 23.9 \\
\hline ESF-HD-169-TEMP22-RTD-65 & 0.24 & 32.41 & -21.43 & 21.43 & 23.8 & 23.8 & 23.8 & 23.8 & 23.8 & 23.8 & 23.8 & 23.8 & 23.8 & 23.8 \\
\hline ESF-HD-169-TEMP22-RTD-66 & 0.24 & 32.41 & -21.73 & 21.73 & 23.9 & 23.9 & 23.9 & 23.9 & 23.8 & 23.9 & 23.9 & 23.9 & 23.9 & 23.9 \\
\hline ESF-HD-169-TEMP22-RTD-67 & 0.25 & 32.42 & -22.03 & 22.03 & 23.9 & 23.9 & 23.9 & 23.9 & 23.9 & 23.9 & 23.9 & 23.9 & 23.9 & 23.9 \\
\hline \multicolumn{15}{|l|}{ BH 170 Gage ID } \\
\hline ESF-HD-170-TEMP23-RTD-1 & 0.75 & 39.31 & 2.54 & 2.54 & 30.4 & 53.4 & 63.2 & 70.1 & 76.2 & 81.2 & 85.6 & 89.7 & 93.2 & 95.1 \\
\hline ESF-HD-170-TEMP23-RTD-2 & 0.75 & 39.30 & 2.83 & 2.83 & 30.4 & 45.4 & 55.0 & 61.6 & 67.6 & 72.4 & 76.7 & 80.6 & 83.8 & 86.3 \\
\hline ESF-HD-170-TEMP23-RTD-3 & 0.75 & 39.30 & 3.13 & 3.13 & 30.2 & 39.2 & 48.1 & 54.5 & 60.2 & 64.8 & 69.0 & 73.0 & 76.0 & 78.5 \\
\hline ESF-HD-170-TEMP23-RTD-4 & 0.75 & 39.30 & 3.43 & 3.43 & 30.1 & 35.6 & 43.5 & 49.4 & 54.8 & 59.2 & 63.2 & 67.1 & 70.2 & 72.7 \\
\hline ESF-HD-170-TEMP23-RTD-5 & 0.75 & 39.29 & 3.72 & 3.72 & 29.8 & 32.9 & 39.4 & 44.7 & 49.7 & 53.9 & 57.7 & 61.5 & 64.6 & 66.9 \\
\hline ESF-HD-170-TEMP23-RTD-6 & 0.75 & 39.29 & 4.02 & 4.02 & 29.5 & 31.1 & 36.3 & 41.0 & 45.6 & 49.5 & 53.1 & 56.8 & 59.8 & 62.2 \\
\hline ESF-HD-170-TEMP23-RTD-7 & 0.75 & 39.29 & 4.31 & 4.31 & 29.2 & 29.9 & 33.8 & 37.8 & 41.9 & 45.5 & 48.9 & 52.3 & 55.3 & 57.6 \\
\hline ESF-HD-170-TEMP23-RTD-8 & 0.75 & 39.29 & 4.61 & 4.61 & 28.9 & 29.1 & 32.0 & 35.4 & 39.1 & 42.3 & 45.5 & 48.7 & 51.5 & 53.7 \\
\hline ESF-HD-170-TEMP23-RTD-9 & 0.75 & 39.28 & 4.90 & 4.90 & 27.6 & 27.8 & 30.2 & 33.0 & 36.1 & 39.2 & 42.0 & 45.1 & 47.7 & 49.8 \\
\hline ESF-HD-170-TEMP23-RTD-10 & 0.75 & 39.28 & 5.20 & 5.20 & 28.1 & 28.1 & 29.4 & 31.7 & 34.4 & 37.0 & 39.6 & 42.4 & 44.9 & 46.9 \\
\hline ESF-HD-170-TEMP23-RTD-11 & 0.75 & 39.28 & 5.50 & 5.50 & 27.8 & 27.7 & 28.6 & 30.3 & 32.6 & 34.8 & 37.1 & 39.7 & 42.0 & 43.8 \\
\hline ESF-HD-170-TEMP23-RTD-12 & 0.75 & 39.27 & 5.79 & 5.79 & 27.4 & 27.4 & 27.9 & 29.2 & 31.1 & 33.0 & 35.1 & 37.4 & 39.5 & 41.1 \\
\hline ESF-HD-170-TEMP23-RTD-13 & 0.75 & 39.27 & 6.09 & 6.09 & 27.1 & 27.1 & 27.4 & 28.4 & 30.0 & 31.6 & 33.4 & 35.5 & 37.4 & 38.9 \\
\hline ESF-HD-170-TEMP23-RTD-14 & 0.75 & 39.27 & 6.38 & 6.38 & 26.7 & 26.8 & 27.0 & 27.7 & 29.0 & 30.4 & 32.0 & 33.8 & 35.6 & 37.0 \\
\hline ESF-HD-170-TEMP23-RTD-15 & 0.75 & 39.27 & 6.68 & 6.68 & 26.5 & 26.5 & 26.6 & 27.2 & 28.2 & 29.4 & 30.7 & 32.3 & 33.9 & 35.1 \\
\hline ESF-HD-170-TEMP23-RTD-16 & 0.75 & 39.26 & 6.98 & 6.98 & 26.1 & 26.1 & 26.3 & 26.7 & 27.5 & 28.5 & 29.6 & 31.1 & 32.4 & 33.6 \\
\hline ESF-HD-170-TEMP23-RTD-17 & 0.75 & 39.26 & 7.27 & 7.27 & 25.9 & 26.0 & 26.0 & 26.3 & 27.0 & 27.8 & 28.7 & 29.9 & 31.2 & 32.2 \\
\hline ESF-HD-170-TEMP23-RTD-18 & 0.75 & 39.26 & 7.57 & 7.57 & 25.7 & 25.7 & 25.8 & 26.0 & 26.5 & 27.2 & 28.0 & 29.1 & 30.1 & 31.0 \\
\hline ESF-HD-170-TEMP23-RTD-19 & 0.75 & 39.26 & 7.86 & 7.86 & 25.4 & 25.4 & 25.5 & 25.7 & 26.1 & 26.6 & 27.3 & 28.2 & 29.1 & 29.9 \\
\hline ESF-HD-170-TEMP23-RTD-20 & 0.75 & 39.25 & 8.16 & 8.16 & 25.2 & 25.3 & 25.4 & 25.5 & 25.8 & 26.2 & 26.8 & 27.6 & 28.4 & 29.1 \\
\hline ESF-HD-170-TEMP23-RTD-21 & 0.75 & 39.25 & 8.46 & 8.46 & 25.1 & 25.1 & 25.2 & 25.3 & 25.6 & 25.9 & 26.4 & 27.0 & 27.7 & 28.3 \\
\hline ESF-HD-170-TEMP23-RTD-22 & 0.75 & 39.25 & 8.75 & 8.75 & 24.9 & 25.0 & 25.1 & 25.2 & 25.4 & 25.6 & 26.0 & 26.6 & 27.2 & 27.7 \\
\hline ESF-HD-170-TEMP23-RTD-23 & 0.75 & 39.24 & 9.05 & 9.05 & 24.8 & 24.8 & 24.9 & 25.0 & 25.1 & 25.4 & 25.7 & 26.1 & 26.7 & 27.1 \\
\hline ESF-HD-170-TEMP23-RTD-24 & 0.75 & 39.24 & 9.34 & 9.34 & 24.6 & 24.6 & 24.7 & 24.8 & 24.9 & 25.1 & 25.4 & 25.8 & 26.2 & 26.6 \\
\hline ESF-HD-170-TEMP23-RTD-25 & 0.75 & 39.24 & 9.64 & 9.64 & 24.5 & 24.5 & 24.6 & 24.7 & 24.8 & 24.9 & 25.2 & 25.5 & 25.8 & 26.2 \\
\hline ESF-HD-170-TEMP23-RTD-26 & 0.75 & 39.24 & 9.93 & 9.93 & 24.3 & 24.4 & 24.5 & 24.5 & 24.6 & 24.8 & 24.9 & 25.2 & 25.5 & 25.8 \\
\hline ESF-HD-170-TEMP23-RTD-27 & 0.75 & 39.23 & 10.23 & 10.23 & 24.2 & 24.3 & 24.4 & 24.4 & 24.5 & 24.6 & 24.8 & 25.0 & 25.3 & 25.5 \\
\hline ESF-HD-170-TEMP23-RTD-28 & 0.75 & 39.23 & 10.53 & 10.53 & 24.2 & 24.3 & 24.3 & 24.3 & 24.4 & 24.5 & 24.6 & 24.8 & 25.0 & 25.2 \\
\hline ESF-HD-170-TEMP23-RTD-29 & 0.75 & 39.23 & 10.82 & 10.82 & 24.0 & 24.1 & 24.1 & 24.2 & 24.2 & 24.3 & 24.4 & 24.5 & 24.7 & 24.9 \\
\hline ESF-HD-170-TEMP23-RTD-30 & 0.75 & 39.22 & 11.12 & 11.12 & 24.0 & 24.0 & 24.1 & 24.1 & 24.2 & 24.2 & 24.3 & 24.4 & 24.6 & 24.7 \\
\hline ESF-HD-170-TEMP23-RTD-31 & 0.75 & 39.22 & 11.41 & 11.41 & 23.9 & 23.9 & 24.0 & 24.0 & 24.1 & 24.1 & 24.2 & 24.3 & 24.4 & 24.6 \\
\hline
\end{tabular}




\begin{tabular}{|c|c|c|c|c|c|c|c|c|c|c|c|c|c|c|}
\hline \multirow{5}{*}{ ESF-HD-170-TEMP23-RTD-32 } & \multicolumn{14}{|c|}{ Temperature Data Obtained from the DST } \\
\hline & \multicolumn{14}{|c|}{ Days after startup } \\
\hline & \multicolumn{4}{|c|}{ As Built Locations (m) } & 0 & 7 & 17 & 27 & 38 & 48 & 58 & 69 & 79 & 87 \\
\hline & $\mathbf{x}$ & y & $\mathbf{z}$ & D & \multicolumn{10}{|c|}{$\left({ }^{\circ} \mathrm{C}\right)$} \\
\hline & 0.75 & 39.22 & 11.71 & 11.71 & 23.8 & 23.8 & 23.9 & 23.9 & 24.0 & 24.0 & 24.1 & 24.2 & 24.3 & 4.4 \\
\hline ESF-HD-170-TEMP23-RTD-33 & 0.75 & 39.22 & 12.01 & 12.01 & 23.7 & 3.7 & 3.8 & 3.8 & 3.9 & 3.9 & 4.0 & 4.0 & 24.1 & 4.2 \\
\hline ESF-HD-170-TEMP23-RTD-34 & 0.75 & 39.21 & 12.30 & 12.30 & 23.7 & 23.8 & 23.8 & 3.8 & 3.9 & 23.9 & 24.0 & 4.0 & 24.1 & 4.2 \\
\hline ESF-HD-170-TEMP23-RT & 0.75 & 39.21 & 12.60 & 12.60 & 23.7 & 23.7 & 23.7 & 23.7 & 23.8 & 23.8 & 23.8 & 23.9 & 24.0 & 4.0 \\
\hline ESF-HD-170-TEMP23-RTD-36 & 0.75 & 39.21 & 12.89 & 12.89 & 23.6 & 23.7 & 23.7 & 23.7 & 23.8 & 23.8 & 23.8 & 23.9 & 24.0 & 24.0 \\
\hline ESF-HD-170-TEMP23-RTD-37 & 0.75 & 39.20 & 13.19 & 13.19 & 23.6 & 23.6 & 23.6 & 23.7 & 23.7 & 23.7 & 23.7 & 23.8 & 23.8 & 3.9 \\
\hline ESF-HD-170-TEMP23-RTD-38 & 0.75 & 39.20 & 13.49 & 13.49 & 23.6 & 23.6 & 23.6 & 23.6 & 23.7 & 23.7 & 23.7 & 23.7 & 23.8 & \\
\hline ESF-HD-170-TEMP23-RTD-39 & 0.75 & 39.20 & 13.78 & 13.78 & 23.5 & 23.6 & 23.6 & 23.6 & 23.6 & 23.6 & 23.7 & 23.7 & 23.7 & 3.8 \\
\hline ESF-HD-170-TEMP23-RTD-40 & 0.75 & 39.20 & 14.08 & 14.08 & 23.5 & 23.5 & 23.5 & 23.5 & 23.6 & 23.6 & 23.6 & 23.6 & 23.7 & 3.7 \\
\hline ESF-HD-170-TEMP23-RTD-41 & 0.75 & 39.19 & 14.37 & 14.37 & 23.5 & 23.5 & 23.5 & 23.5 & 23.5 & 23.6 & 23.6 & 23.6 & 23.6 & \\
\hline ESF-HD-170-TEMP23-RTD-42 & 0.75 & 39.19 & 14.67 & 14.67 & 23.5 & 23.5 & 23.5 & 23.5 & 23.5 & 23.5 & 23 & 23.6 & 23.6 & \\
\hline ESF-HD-170-TEMP23-RTD-43 & 0.75 & 39.19 & 14.96 & 14.96 & 23.4 & 23.4 & 23.5 & 20.5 & 23.5 & 23.5 & & 23.5 & 23.6 & \\
\hline -RTD-44 & 0.75 & 39.18 & 15.26 & 15.26 & 23.4 & 23.5 & 23.5 & 20.5 & 23.5 & 23.5 & 2.0 & 23.5 & 23.6 & \\
\hline -TEMP23-RTD-45 & 0.75 & 39.18 & 15.56 & 15.56 & 23.4 & 23.4 & 23.4 & 20.4 & 23.4 & 23.4 & 23.4 & 23.4 & 23.5 & 23.5 \\
\hline -TEMP23-RTD-46 & 0.75 & 39.18 & 15.85 & 15.85 & 23.4 & 23.4 & 23.4 & 23.4 & 23.4 & 23.4 & 23.4 & 23.5 & 23.5 & 23.5 \\
\hline ESF-HD-17 & 0.75 & 39.18 & 16.15 & 16.15 & 23.3 & 23.3 & 23.3 & 23.4 & 23.4 & 23.4 & & 23.4 & 23.4 & \\
\hline ESF-HD-1 & 0.75 & 9.17 & 16.44 & 16.44 & 23.4 & 23.4 & 20.4 & 20.4 & 23.4 & 23.4 & & 3.4 & 23.4 & 3.4 \\
\hline ESF-H & 0.75 & 9.17 & 16.74 & 6.74 & 23.3 & & 0.5 & 0.2 & 3.2 & & & 3.4 & 23.4 & \\
\hline ESF-H & 0.75 & 39.17 & 7.04 & 17 & 23.4 & 23.4 & & 0.4 & 3.4 & 2 & & 3.4 & 23.4 & \\
\hline ESF-H & .75 & & & & 3.3 & & & & & & & & 3.4 & \\
\hline-52 & & 3 & & & & & & & & & & & & \\
\hline ESF-HD-170-TEMP23-RTD-53 & & & & & & & & & & & & 3.3 & 23.3 & \\
\hline ESF-HD-170-TEMP23-RTD-54 & & 9.16 & 18.22 & 18.22 & 23.3 & & & & & & & 0.0 & 23.3 & \\
\hline ESF-HD-170-TEMP23-RTD-55 & 0.75 & 39.15 & 18.51 & 18.51 & & & & & & & & & & \\
\hline ESF-HD-170-TEMP23-RTD-56 & 0.75 & & & 18.81 & & & & & & & & & & \\
\hline ESF-HD-170-TEMP23-RTD-57 & 0.75 & 39.15 & 19.11 & 19.11 & & & & & & & & & & \\
\hline ESF-HD-170-TEMP23-RTD-58 & 0.75 & 39.15 & 19.40 & 19.40 & & & & & & & & & & \\
\hline & 0.75 & 39.14 & 19.70 & 19.70 & & & & & & & & & & \\
\hline ESF-HD-170-TEMP23-RTD-60 & 0.75 & 39.14 & 19.99 & 19.99 & & & & & 23.3 & & & 23.3 & 23.3 & \\
\hline ESF-HD-170-TEMP23-RTD-61 & 0.75 & 39.14 & 20.29 & 20.29 & 23.0 & & & 23.2 & 23.2 & & & 23.2 & 23.2 & \\
\hline ESF-HD-170-TEMP23-RTD-62 & 0.75 & 39.13 & 20.59 & 20.59 & 23.2 & & & & 23.2 & & & 23.2 & 23.2 & \\
\hline ESF-HD-170-TEMP23-RTD-63 & 0.75 & 39.13 & 20.88 & 20.88 & 23.2 & 23.2 & & & 23.2 & 23.2 & & 23.2 & 23.2 & \\
\hline ESF-HD-170-TEMP23-RTD-64 & 0.75 & 39.13 & 21.18 & 21.18 & 23.2 & 23.2 & & 23.2 & 23.2 & & & 23.2 & 23.2 & \\
\hline ESF-HD-170-TEMP23-RTD-65 & 0.75 & 39.13 & 21.47 & 21.47 & 23.2 & & & & 23.2 & & & 23.2 & 23.2 & \\
\hline ESF-HD-170-TEMP23-RTD-66 & 0.75 & 39.12 & 21.77 & 21.77 & 23.2 & & 23.2 & & 23.2 & & 23.2 & 23.2 & 23.2 & 23.2 \\
\hline ESF-HD-170-TEMP23-F & 0.75 & 39.12 & 22.07 & 22.07 & 23.1 & 23.1 & & & 23.2 & & & 23.2 & 23.1 & \\
\hline
\end{tabular}


First Quarter Report of Temperature Measurements During the Heating Phase of the Drift-Scale Test

\begin{tabular}{|c|c|c|c|c|c|c|c|c|c|c|c|c|c|c|}
\hline \multirow{5}{*}{\begin{tabular}{|l} 
ESF-HD-171-TEMP24-RTD-1 \\
\end{tabular}} & \multicolumn{14}{|c|}{ Temperature Data Obtained from the DST } \\
\hline & \multicolumn{14}{|c|}{ Days after startup } \\
\hline & \multicolumn{4}{|c|}{ As Built Locations (m) } & 0 & 7 & 17 & 27 & 38 & 48 & 58 & 69 & 79 & 87 \\
\hline & $\mathbf{x}$ & $\mathbf{y}$ & $\mathbf{z}$ & D & \multicolumn{10}{|c|}{$\left({ }^{\circ} \mathbf{C}\right)$} \\
\hline & -1.92 & 39.31 & 1.89 & 2.69 & 30.4 & 52.1 & 61.9 & 68.9 & 75.2 & 80.4 & 84.9 & 89.0 & 92.6 & 94.5 \\
\hline ESF-HD-171-TEMP24-RTD-2 & -2.13 & 39.31 & 2.10 & 2.99 & 30.4 & 43.4 & 53.0 & 60.0 & 66.3 & 71.5 & 76.1 & 80.4 & 83.9 & 86.4 \\
\hline ESF-HD-171-TEMP24-RTD-3 & -2.34 & 39.31 & 2.31 & 3.29 & 30.2 & 38.5 & 47.4 & 54.1 & 60.4 & 65.5 & 70.0 & 74.5 & 78.0 & 80.6 \\
\hline ESF-HD-171-TEMP24-RTD-4 & -2.54 & 39.31 & 2.51 & 3.57 & 30.0 & 34.8 & 42.6 & 48.9 & 55.0 & 59.9 & 64.4 & 68.8 & 72.3 & 74.9 \\
\hline ESF-HD-171-TEMP24-RTD-5 & -2.75 & 39.31 & 2.72 & 3.87 & 29.8 & 32.6 & 39.3 & 45.1 & 50.9 & 55.7 & 60.1 & 64.4 & 68.0 & 70.6 \\
\hline ESF-HD-171-TEMP24-RTD-6 & -2.96 & 39.31 & 2.93 & 4.16 & 29.5 & 31.0 & 36.2 & 41.5 & 46.8 & 51.3 & 55.5 & 59.7 & 63.3 & 65.9 \\
\hline ESF-HD-171-TEMP24-RTD-7 & -3.16 & 39.31 & 3.14 & 4.45 & 29.2 & 29.9 & 33.9 & 38.5 & 43.4 & 47.7 & 51.8 & 55.9 & 59.4 & 62.0 \\
\hline ESF-HD-171-TEMP24-RTD-8 & -3.37 & 39.31 & 3.35 & 4.75 & 28.8 & 29.1 & 32.0 & 35.8 & 40.2 & 44.2 & 48.0 & 52.0 & 55.3 & 57.9 \\
\hline ESF-HD-171-TEMP24-RTD-9 & -3.58 & 39.31 & 3.56 & 5.05 & 28.5 & 28.4 & 30.5 & 33.7 & 37.6 & 41.3 & 44.9 & 48.7 & 51.9 & 54.5 \\
\hline ESF-HD-171-TEMP24-RTD-10 & -3.79 & 39.31 & 3.77 & 5.35 & 28.1 & 28.1 & 29.4 & 32.0 & 35.4 & 38.7 & 42.1 & 45.6 & 48.8 & 51.2 \\
\hline ESF-HD-171-TEMP24-RTD-11 & -3.99 & 39.31 & 3.98 & 5.64 & 27.7 & 27.7 & 28.6 & 30.6 & 33.6 & 36.6 & 39.7 & 43.1 & 46.1 & 48.4 \\
\hline ESF-HD-171-TEMP24-RTD-12 & -4.20 & 39.31 & 4.19 & 5.93 & 27.4 & 27.3 & 27.9 & 29.5 & 32.0 & 34.7 & & 40.6 & 43.4 & 45.6 \\
\hline ESF-HD-171-TEMP24-RTD-13 & -4.41 & 39.31 & 4.40 & 6.23 & 27.0 & 27.0 & 27.4 & 28.6 & 30.8 & 33.2 & 35.7 & 38.6 & 41.3 & 43.4 \\
\hline ESF-HD-171-TEMP24-RTD-14 & -4.61 & 39.31 & 4.61 & 6.52 & 26.7 & 26.7 & 26.9 & 27.9 & 29.7 & 31.7 & 34.1 & 36.7 & 39.2 & 41.2 \\
\hline ESF-HD-171-TEMP24-RTD-15 & -4.82 & 39.31 & 4.81 & 6.81 & 26.4 & 26.5 & 26.6 & 27.3 & 28.8 & 30.6 & 32.7 & 35.1 & 37.5 & 39.3 \\
\hline ESF-HD-171-TEMP24-RTD-16 & -5.03 & 39.31 & 5.02 & 7.11 & 26.1 & 26.2 & 26.3 & 26.8 & 28.0 & 29.5 & 31.3 & 33.5 & 35.7 & 37.4 \\
\hline ESF-HD-171-TEMP24-RTD-17 & -5.24 & 39.31 & 5.23 & 7.40 & 25.9 & 25.9 & 26.0 & 26.4 & 27.4 & 28.7 & 30.3 & 32.3 & 34.3 & 35.9 \\
\hline ESF-HD-171-TEMP24-RTD-18 & -5.44 & 39.31 & 5.44 & 7.69 & 25.6 & 25.7 & 25.8 & 26.1 & 26.9 & 28.0 & 29.4 & 31.3 & 33.1 & 34.6 \\
\hline ESF-HD-171-TEMP24-RTD-19 & -5.65 & 39.31 & 5.65 & 7.99 & 25.4 & 25.5 & 25.6 & 25.8 & 26.4 & 27.4 & 28.6 & 30.2 & 31.8 & 33.2 \\
\hline ESF-HD-171-TEMP24-RTD-20 & -5.86 & 39.31 & 5.86 & 8.29 & 25.2 & 25.3 & 25.3 & 25.5 & 26.0 & 26.8 & 27.9 & 29.3 & 30.8 & 32.0 \\
\hline ESF-HD-171-TEMP24-RTD-21 & -6.06 & 39.31 & 6.07 & 8.58 & 25.0 & 25.1 & 25.2 & 25.3 & 25.7 & 26.4 & 27.3 & 28.6 & 29.9 & 31.1 \\
\hline ESF-HD-171-TEMP24-RTD-22 & -6.27 & 39.31 & 6.28 & 8.87 & 20.9 & 21.0 & 21.0 & 21.1 & 21.4 & 21.9 & 22.7 & 23.7 & 24.8 & 25.8 \\
\hline ESF-HD-171-TEMP24-RTD-23 & -6.48 & 39.31 & 6.49 & 9.17 & 24.6 & 24.7 & 24.8 & 24.9 & 25.2 & 25.6 & 26.3 & 27.3 & 28.3 & 29.3 \\
\hline ESF-HD-171-TEMP24-RTD-24 & -6.69 & 39.31 & 6.70 & 9.47 & 24.2 & 24.3 & 24.3 & 24.5 & 24.8 & 25.2 & 25.8 & 26.7 & 27.6 & 28.5 \\
\hline ESF-HD-171-TEMP24-RTD-25 & -6.89 & 39.31 & 6.91 & 9.76 & 24.4 & 24.5 & 24.5 & 24.6 & 24.8 & 25.1 & 25.6 & 26.3 & 27.1 & 27.9 \\
\hline ESF-HD-171-TEMP24-RTD-26 & -7.10 & 39.30 & 7.11 & 10.05 & 24.4 & 24.4 & 24.5 & 24.5 & 24.7 & 24.9 & 25.3 & 26.0 & 26.7 & 27.4 \\
\hline ESF-HD-171-TEMP24-RTD-27 & -7.31 & 39.30 & 7.32 & 10.35 & 24.2 & 24.3 & 24.3 & 24.4 & 24.5 & 24.7 & 25.1 & 25.6 & 26.3 & 26.9 \\
\hline ESF-HD-171-TEMP24-RTD-28 & -7.51 & 39.30 & 7.53 & 10.64 & 22.6 & 22.6 & 22.6 & 22.6 & 22.7 & 22.8 & 23.0 & 23.4 & 23.9 & 24.5 \\
\hline ESF-HD-171-TEMP24-RTD-29 & -7.72 & 39.30 & 7.74 & 10.93 & 24.1 & 24.1 & 24.2 & 24.2 & 24.3 & 24.5 & 24.7 & 25.1 & 25.6 & 26.1 \\
\hline ESF-HD-171-TEMP24-RTD-30 & -7.93 & 39.30 & 7.95 & 11.23 & 22.2 & 23.4 & 23.8 & 24.0 & 24.1 & 24.2 & 24.4 & 24.9 & 25.3 & 25.7 \\
\hline ESF-HD-171-TEMP24-RTD-31 & -8.14 & 39.30 & 8.16 & 11.53 & 23.9 & 23.9 & 24.0 & 24.0 & 24.1 & 24.2 & 24.4 & 24.7 & 25.0 & 25.4 \\
\hline ESF-HD-171-TEMP24-RTD-32 & -8.34 & 39.30 & 8.37 & 11.82 & 23.9 & 23.9 & 23.9 & 24.0 & 24.0 & 24.1 & 24.3 & 24.5 & 24.9 & 25.2 \\
\hline ESF-HD-171-TEMP24-RTD-33 & -8.55 & 39.30 & 8.58 & 12.11 & 23.8 & 23.8 & 23.9 & 23.9 & 24.0 & 24.0 & 24.2 & 24.4 & 24.7 & 24.9 \\
\hline ESF-HD-171-TEMP24-RTD-34 & -8.76 & 39.30 & 8.79 & 12.41 & 23.8 & 23.8 & 23.8 & 23.9 & 23.9 & 24.0 & 24.1 & 24.3 & 24.5 & 24.7 \\
\hline ESF-HD-171-TEMP24-RTD-35 & -8.96 & 39.30 & 9.00 & 12.70 & 23.7 & 23.7 & 23.7 & 23.8 & 23.8 & 23.8 & 23.9 & 24.1 & 24.3 & 24.5 \\
\hline ESF-HD-171-TEMP24-RTD-36 & -9.17 & 39.30 & 9.21 & 13.00 & 23.7 & 23.7 & 23.7 & 23.7 & 23.8 & 23.8 & 23.9 & 24.0 & 24.2 & 24.4 \\
\hline ESF-HD-171-TEMP24-RTD-37 & -9.38 & 39.30 & 9.42 & 13.29 & 23.6 & 23.7 & 23.7 & 23.7 & 23.7 & 23.8 & 23.8 & 24.0 & 24.1 & 24.3 \\
\hline
\end{tabular}




\begin{tabular}{|c|c|c|c|c|c|c|c|c|c|c|c|c|c|c|}
\hline & \multicolumn{14}{|c|}{ Temperature Data Obtained from the DST } \\
\hline & \multicolumn{14}{|c|}{ Days after startup } \\
\hline & \multicolumn{4}{|c|}{ As Built Locations (m) } & 0 & 7 & 17 & 27 & 38 & 48 & 58 & 69 & 79 & 87 \\
\hline & $\mathbf{x}$ & $\mathbf{y}$ & $\mathbf{z}$ & D & \multicolumn{10}{|c|}{$\left({ }^{\circ} \mathbf{C}\right)$} \\
\hline ESF-HD-171-TEMP24-RTD-38 & -9.59 & 39.30 & 9.62 & 13.58 & 23.6 & 23.6 & 23.6 & 23.7 & 23.7 & 23.7 & 23.8 & 23.9 & 24.0 & 24.1 \\
\hline ESF-HD-171-TEMP24-RTD-39 & -9.79 & 39.30 & 9.83 & 13.87 & 23.6 & 23.6 & 23.6 & 23.6 & 23.7 & 23.7 & 23.7 & 23.8 & 23.9 & 24.0 \\
\hline ESF-HD-171-TEMP24-RTD-40 & -10.00 & 39.30 & 10.04 & 14.17 & 23.6 & 23.5 & 23.6 & 23.6 & 23.6 & 23.6 & 23.6 & 23.8 & 23.9 & 24.0 \\
\hline ESF-HD-171-TEMP24-RTD-41 & -10.21 & 39.30 & 10.25 & 14.47 & 23.5 & 23.5 & 23.5 & 23.6 & 23.6 & 23.6 & 23.6 & 23.7 & 23.8 & 23.9 \\
\hline ESF-HD-171-TEMP24-RTD-42 & -10.41 & 39.30 & 10.46 & 14.76 & 23.5 & 23.5 & 23.5 & 23.6 & 23.6 & 23.6 & 23.6 & 23.7 & 23.8 & 23.8 \\
\hline ESF-HD-171-TEMP24-RTD-43 & -10.62 & 39.30 & 10.67 & 15.05 & 23.5 & 23.5 & 23.5 & 23.5 & 23.5 & 23.6 & 23.6 & 23.6 & 23.7 & 23.8 \\
\hline ESF-HD-171-TEMP24-RTD-44 & -10.83 & 39.30 & 10.88 & 15.35 & 23.5 & 23.5 & 23.5 & 23.5 & 23.5 & 23.5 & 23.5 & 23.6 & 23.6 & 23.7 \\
\hline ESF-HD-171-TEMP24-RTD-45 & -11.04 & 39.30 & 11.09 & 15.65 & 23.4 & 23.5 & 23.5 & 23.5 & 23.5 & 23.5 & 23.5 & 23.6 & 23.6 & 23.6 \\
\hline ESF-HD-171-TEMP24-RTD-46 & -11.24 & 39.30 & 11.30 & 15.94 & 23.4 & 23.4 & 23.4 & 23.4 & 23.4 & 23.5 & 23.5 & 23.5 & 23.5 & 23.6 \\
\hline ESF-HD-171-TEMP24-RTD-47 & -11.45 & 39.30 & 11.51 & 16.24 & 23.4 & 23.4 & 23.4 & 23.5 & 23.5 & 23.5 & 23.5 & 23.5 & 23.6 & 23.6 \\
\hline ESF-HD-171-TEMP24-RTD-48 & -11.66 & 39.30 & 11.72 & 16.53 & 23.4 & 23.4 & 23.4 & 23.4 & 23.4 & 23.5 & 23.5 & 23.5 & 23.5 & 23.5 \\
\hline ESF-HD-171-TEMP24-RTD-49 & -11.86 & 39.30 & 11.92 & 16.82 & 23.4 & 23.4 & 23.4 & 23.4 & 23.5 & 23.5 & 23.5 & 23.5 & 23.5 & 23.5 \\
\hline ESF-HD-171-TEMP24-RTD-50 & -12.07 & 39.30 & 12.13 & 17.11 & 23.2 & 23.3 & 23.4 & 23.4 & 23.4 & 23.4 & 23.4 & 23.4 & 23.5 & 23.5 \\
\hline ESF-HD-171-TEMP24-RTD-51 & -12.28 & 39.30 & 12.34 & 17.41 & 23.4 & 23.4 & 23.4 & 23.4 & 23.4 & 23.4 & 23.4 & 23.4 & 23.4 & 23.5 \\
\hline ESF-HD-171-TEMP24-RTD-52 & -12.49 & 39.30 & 12.55 & 17.71 & 23.4 & 23.4 & 23.4 & 23.4 & 23.4 & 23.4 & 23.4 & 23.4 & 23.4 & 23.5 \\
\hline ESF-HD-171-TEMP24-RTD-53 & -12.69 & 39.30 & 12.76 & 18.00 & 23.4 & 23.4 & 23.4 & 23.4 & 23.4 & 23.4 & 23.4 & 23.4 & 23.4 & 23.5 \\
\hline ESF-HD-171-TEMP24-RTD-54 & -12.90 & 39.30 & 12.97 & 18.29 & 23.4 & 23.4 & 23.4 & 23.4 & 23.4 & 23.4 & 23.4 & 23.4 & 23.4 & 23.4 \\
\hline ESF-HD-171-TEMP24-RTD-55 & -13.11 & 39.30 & 13.18 & 18.59 & 23.4 & 23.4 & 23.4 & 23.3 & 23.4 & 23.4 & 23.4 & 23.4 & 23.4 & 23.4 \\
\hline ESF-HD-171-TEMP24-RTD-56 & -13.31 & 39.30 & 13.39 & 18.88 & 23.2 & 23.3 & 23.4 & 23.4 & 23.4 & 23.4 & 23.4 & 23.4 & 23.4 & 23.4 \\
\hline ESF-HD-171-TEMP24-RTD-57 & -13.52 & 39.30 & 13.60 & 19.18 & 23.3 & 23.3 & 23.4 & 23.4 & 23.4 & 23.4 & 23.4 & 23.4 & 23.4 & 23.4 \\
\hline ESF-HD-171-TEMP24-RTD-58 & -13.73 & 39.29 & 13.81 & 19.47 & 23.3 & 23.3 & 23.3 & 23.4 & 23.4 & 23.4 & 23.4 & 23.4 & 23.4 & 23.4 \\
\hline ESF-HD-171-TEMP24-RTD-59 & -13.94 & 39.29 & 14.02 & 19.77 & 23.3 & 23.2 & 23.3 & 23.3 & 23.3 & 23.4 & 23.4 & 23.4 & 23.4 & 23.4 \\
\hline ESF-HD-171-TEMP24-RTD-60 & -14.14 & 39.29 & 14.22 & 20.05 & 23.3 & 23.3 & 23.3 & 23.3 & 23.3 & 23.3 & 23.3 & 23.3 & 23.3 & 23.4 \\
\hline ESF-HD-171-TEMP24-RTD-61 & -14.35 & 39.29 & 14.43 & 20.35 & 22.4 & 23.3 & 23.3 & 23.3 & 23.3 & 23.3 & 23.3 & 23.3 & 23.3 & 23.3 \\
\hline ESF-HD-171-TEMP24-RTD-62 & -14.56 & 39.29 & 14.64 & 20.65 & 23.2 & 23.3 & 23.3 & 23.3 & 23.3 & 23.3 & 23.4 & 23.4 & 23.4 & 23.4 \\
\hline ESF-HD-171-TEMP24-RTD-63 & -14.76 & 39.29 & 14.85 & 20.94 & 23.3 & 23.3 & 23.3 & 23.3 & 23.3 & 23.4 & 23.4 & 23.4 & 23.4 & 23.4 \\
\hline ESF-HD-171-TEMP24-RTD-64 & -14.97 & 39.29 & 15.06 & 21.24 & 23.3 & 23.3 & 23.3 & 23.3 & 23.3 & 23.3 & 23.3 & 23.4 & 23.4 & 23.4 \\
\hline ESF-HD-171-TEMP24-RTD-65 & -15.18 & 39.29 & 15.27 & 21.53 & 23.3 & 23.3 & 23.3 & 23.3 & 23.3 & 23.3 & 23.3 & 23.3 & 23.4 & 23.4 \\
\hline ESF-HD-171-TEMP24-RTD-66 & -15.39 & 39.29 & 15.48 & 21.83 & 23.3 & 23.3 & 23.3 & 23.3 & 23.3 & 23.3 & 23.4 & 23.4 & 23.4 & 23.4 \\
\hline ESF-HD-171-TEMP24-RTD-67 & -15.59 & 39.29 & 15.69 & 22.12 & 23.3 & 23.3 & 23.3 & 23.3 & 23.3 & 23.3 & 23.3 & 23.4 & 23.4 & 23.4 \\
\hline \multicolumn{15}{|l|}{ BH 172 Gage ID } \\
\hline ESF-HD-172-TEMP25-RTD-1 & -1.65 & 39.29 & -1.64 & 2.33 & 30.6 & 41.6 & 50.8 & 58.2 & 64.3 & 69.5 & 74.1 & 78.5 & 82.4 & 84.7 \\
\hline ESF-HD-172-TEMP25-RTD-2 & -1.86 & 39.28 & -1.85 & 2.62 & 24.5 & 31.2 & 39.4 & 46.5 & 53.4 & 59.2 & 63.9 & 78.5 & 82.4 & 84.7 \\
\hline ESF-HD-172-TEMP25-RTD-3 & -2.08 & 39.28 & -2.07 & 2.93 & 30.4 & 35.3 & 42.8 & 49.3 & 55.1 & 60.0 & 64.4 & 68.8 & 72.4 & 75.0 \\
\hline ESF-HD-172-TEMP25-RTD-4 & -2.29 & 39.27 & -2.28 & 3.23 & 29.8 & 32.4 & 38.9 & 44.8 & 50.4 & 55.1 & 59.4 & 63.7 & 67.3 & 69.9 \\
\hline ESF-HD-172-TEMP25-RTD-5 & -2.50 & 39.26 & -2.49 & 3.53 & 29.7 & 31.1 & 36.1 & 41.3 & 46.6 & 51.1 & 55.3 & 59.6 & 63.1 & 65.8 \\
\hline ESF-HD-172-TEMP25-RTD-6 & -2.71 & 39.26 & -2.70 & 3.83 & 29.4 & 30.0 & 33.6 & 38.1 & 42.9 & 47.2 & 51.2 & 55.4 & 58.8 & 61.6 \\
\hline
\end{tabular}


First Quarter Report of Temperature Measurements During the Heating Phase of the Drift-Scale Test

\begin{tabular}{|c|c|c|c|c|c|c|c|c|c|c|c|c|c|c|}
\hline \multirow{5}{*}{\begin{tabular}{|l} 
\\
ESF-HD-172-TEMP25-RTD-7 \\
\end{tabular}} & \multicolumn{14}{|c|}{ Temperature Data Obtained from the DST } \\
\hline & \multicolumn{14}{|c|}{ Days after startup } \\
\hline & \multicolumn{4}{|c|}{ As Built Locations (m) } & 0 & 7 & 17 & 27 & 38 & 48 & 58 & 69 & 79 & 87 \\
\hline & $\mathbf{x}$ & $\mathbf{y}$ & $\mathbf{z}$ & D & \multicolumn{10}{|c|}{$\left({ }^{\circ} \mathbf{C}\right)$} \\
\hline & -2.93 & 39.25 & -2.92 & 4.14 & 28.5 & 28.9 & 31.9 & 35.9 & 40.4 & 44.4 & 48.3 & 52.3 & 55.7 & 58.4 \\
\hline ESF-HD-172-TEMP25-RTD-8 & -3.14 & 39.25 & -3.13 & 4.43 & 27.6 & 27.5 & 30.1 & 33.5 & 36.4 & 40.7 & 45.0 & 49.3 & 52.3 & 55.2 \\
\hline ESF-HD-172-TEMP25-RTD-9 & -3.35 & 39.24 & -3.34 & 4.73 & 28.2 & 28.2 & 29.6 & 32.4 & 36.0 & 39.5 & 43.0 & 46.7 & 49.9 & 52.4 \\
\hline ESF-HD-172-TEMP25-RTD-10 & -3.56 & 39.23 & -3.55 & 5.03 & 28.0 & 27.9 & 28.8 & 31.0 & 34.2 & 37.3 & 40.5 & 44.0 & 47.1 & 49.5 \\
\hline ESF-HD-172-TEMP25-RTD-11 & -3.77 & 39.23 & -3.77 & 5.33 & 27.6 & 27.6 & 28.2 & 30.0 & 32.8 & 35.7 & 38.7 & 42.0 & 45.0 & 47.3 \\
\hline ESF-HD-172-TEMP25-RTD-12 & -3.99 & 39.22 & -3.98 & 5.64 & 27.3 & 27.3 & 27.7 & 29.1 & 31.5 & 34.1 & 36.9 & 40.0 & 42.8 & 45.0 \\
\hline ESF-HD-172-TEMP25-RTD-13 & -4.20 & 39.21 & -4.19 & 5.93 & 27.0 & 27.0 & 27.3 & 28.4 & 30.5 & 32.9 & 35.4 & 38.3 & 41.0 & 43.2 \\
\hline ESF-HD-172-TEMP25-RTD-14 & -4.41 & 39.21 & -4.40 & 6.23 & 26.7 & 26.7 & 26.9 & 27.7 & 29.5 & 31.6 & 33.9 & 36.6 & 39.1 & 41.1 \\
\hline ESF-HD-172-TEMP25-RTD-15 & -4.62 & 39.20 & -4.62 & 6.53 & 26.4 & 26.4 & 26.6 & 27.3 & 28.6 & 30.4 & 32.7 & 35.3 & 37.6 & 39.6 \\
\hline ESF-HD-172-TEMP25-RTD-16 & -4.84 & 39.20 & -4.83 & 6.84 & 26.1 & 26.2 & 26.3 & 26.8 & 28.1 & 29.7 & 31.6 & 33.9 & 36.1 & 37.9 \\
\hline ESF-HD-172-TEMP25-RTD-17 & -5.05 & 39.19 & -5.04 & 7.13 & 25.9 & 25.9 & 26.0 & 26.4 & 27.4 & 28.9 & 30.6 & 32.8 & 34.8 & 36.5 \\
\hline ESF-HD-172-TEMP25-RTD-18 & -5.26 & 39.18 & -5.25 & 7.43 & 25.6 & 25.7 & 25.8 & 26.1 & 27.0 & 28.2 & 29.8 & 31.7 & 33.6 & 35.2 \\
\hline ESF-HD-172-TEMP25-RTD-19 & -5.47 & 39.18 & -5.46 & 7.73 & 25.5 & 25.5 & 25.6 & 25.9 & 26.6 & 27.7 & 29.1 & 30.8 & 32.6 & 34.1 \\
\hline ESF-HD-172-TEMP25-RTD-20 & -5.68 & 39.17 & -5.68 & 8.03 & 25.3 & 25.3 & 25.4 & 25.6 & 26.2 & 27.1 & 28.3 & 29.9 & 31.5 & 32.9 \\
\hline ESF-HD-172-TEMP25-RTD-21 & -5.90 & 39.16 & -5.89 & 8.34 & 23.4 & 20.1 & 21.3 & 23.8 & 19.7 & 22.6 & 25.7 & 27.8 & 28.9 & 30.1 \\
\hline ESF-HD-172-TEMP25-RTD-22 & -6.11 & 39.16 & -6.10 & 8.63 & 24.9 & 25.0 & 24.9 & 25.2 & 25.3 & 26.2 & 27.2 & 28.4 & 29.8 & 30.9 \\
\hline ESF-HD-172-TEMP25-RTD-23 & -6.32 & 39.15 & -6.31 & 8.93 & 24.8 & 24.5 & 25.1 & 25.4 & 25.8 & 26.6 & 27.7 & 29.1 & 30.6 & 31.8 \\
\hline ESF-HD-172-TEMP25-RTD-24 & -6.53 & 39.15 & -6.53 & 9.23 & 24.7 & 24.7 & 24.8 & 24.9 & 25.1 & 25.6 & 26.3 & 27.2 & 28.3 & 29.2 \\
\hline ESF-HD-172-TEMP25-RTD-25 & -6.75 & 39.14 & -6.74 & 9.54 & 24.6 & 24.6 & 24.7 & 24.8 & 25.0 & 25.4 & 25.9 & 26.8 & 27.8 & 28.6 \\
\hline ESF-HD-172-TEMP25-RTD-26 & -6.96 & 39.13 & -6.95 & 9.84 & 24.5 & 24.5 & 24.6 & 24.6 & 24.8 & 25.1 & 25.6 & 26.4 & 27.2 & 28.0 \\
\hline ESF-HD-172-TEMP25-RTD-27 & -7.17 & 39.13 & -7.16 & 10.13 & 24.3 & 23.3 & 24.3 & 24.6 & 24.6 & 24.9 & 25.4 & 26.1 & 26.8 & 27.5 \\
\hline ESF-HD-172-TEMP25-RTD-28 & -7.38 & 39.12 & -7.38 & 10.44 & 24.3 & 24.3 & 24.4 & 24.4 & 24.5 & 24.8 & 25.1 & 25.7 & 26.4 & 27.0 \\
\hline ESF-HD-172-TEMP25-RTD-29 & -7.59 & 39.12 & -7.59 & 10.73 & 24.2 & 24.3 & 24.3 & 24.4 & 24.5 & 24.7 & 25.0 & 25.5 & 26.1 & 26.7 \\
\hline ESF-HD-172-TEMP25-RTD-30 & -7.81 & 39.11 & -7.80 & 11.04 & 24.2 & 24.2 & 24.2 & 24.3 & 24.4 & 24.5 & 24.8 & 25.2 & 25.7 & 26.3 \\
\hline ESF-HD-172-TEMP25-RTD-31 & -8.02 & 39.10 & -8.01 & 11.34 & 24.1 & 24.1 & 24.1 & 24.2 & 23.9 & 24.1 & 24.5 & 25.0 & 25.4 & 26.0 \\
\hline ESF-HD-172-TEMP25-RTD-32 & -8.23 & 39.10 & -8.23 & 11.64 & 24.1 & 24.1 & 24.1 & 24.2 & 24.2 & 24.3 & 24.5 & 24.8 & 25.2 & 25.6 \\
\hline ESF-HD-172-TEMP25-RTD-33 & -8.44 & 39.09 & -8.44 & 11.94 & 21.4 & 19.5 & 24.0 & 23.5 & 22.7 & 23.8 & 24.3 & 24.6 & 25.0 & 25.3 \\
\hline ESF-HD-172-TEMP25-RTD-34 & -8.66 & 39.08 & -8.65 & 12.24 & 24.0 & 23.9 & 24.0 & 24.0 & 24.1 & 24.1 & 24.3 & 24.5 & 24.8 & 25.1 \\
\hline ESF-HD-172-TEMP25-RTD-35 & -8.87 & 39.08 & -8.86 & 12.54 & 23.9 & 23.9 & 24.0 & 24.0 & 24.0 & 24.1 & 24.2 & 24.4 & 24.6 & 24.9 \\
\hline ESF-HD-172-TEMP25-RTD-36 & -9.08 & 39.07 & -9.08 & 12.84 & 23.9 & 23.9 & 23.9 & 24.0 & 24.0 & 24.0 & 24.1 & 24.3 & 24.5 & 24.7 \\
\hline ESF-HD-172-TEMP25-RTD-37 & -9.29 & 39.07 & -9.29 & 13.14 & 23.9 & 23.9 & 23.9 & 24.0 & 24.0 & 24.0 & 24.1 & 24.2 & 24.4 & 24.6 \\
\hline ESF-HD-172-TEMP25-RTD-38 & -9.50 & 39.06 & -9.50 & 13.44 & 23.9 & 23.9 & 23.9 & 23.9 & 24.0 & 24.0 & 24.0 & 24.2 & 24.3 & 24.5 \\
\hline ESF-HD-172-TEMP25-RTD-39 & -9.72 & 39.05 & -9.71 & 13.74 & 23.7 & 23.6 & 23.8 & 23.8 & 23.9 & 23.9 & 23.9 & 24.0 & 24.2 & 24.3 \\
\hline ESF-HD-172-TEMP25-RTD-40 & -9.93 & 39.05 & -9.92 & 14.04 & 23.8 & 23.8 & 23.8 & 23.8 & 23.9 & 23.9 & 23.9 & 24.0 & 24.1 & 24.2 \\
\hline ESF-HD-172-TEMP25-RTD-41 & -10.14 & 39.04 & -10.14 & 14.34 & 23.8 & 23.8 & 23.8 & 23.9 & 23.9 & 23.9 & 23.9 & 24.0 & 24.1 & 24.2 \\
\hline ESF-HD-172-TEMP25-RTD-42 & -10.35 & 39.04 & -10.35 & 14.64 & 23.8 & 23.8 & 23.8 & 23.9 & 23.9 & 23.9 & 23.9 & 24.0 & 24.1 & 24.2 \\
\hline ESF-HD-172-TEMP25-RTD-43 & -10.57 & 39.03 & -10.56 & 14.94 & 23.7 & 23.6 & 23.7 & 23.7 & 23.8 & 23.8 & 23.8 & 23.8 & 23.9 & 24.0 \\
\hline
\end{tabular}




\begin{tabular}{|c|c|c|c|c|c|c|c|c|c|c|c|c|c|c|}
\hline & \multicolumn{14}{|c|}{ Temperature Data Obtained from the DST } \\
\hline & \multicolumn{14}{|c|}{ Days after startup } \\
\hline & \multicolumn{4}{|c|}{ As Built Locations (m) } & 0 & 7 & 17 & 27 & 38 & 48 & 58 & 69 & 79 & 87 \\
\hline & $\mathbf{x}$ & $\mathbf{y}$ & $\mathbf{z}$ & D & \multicolumn{10}{|c|}{$\left({ }^{\circ} \mathbf{C}\right)$} \\
\hline ESF-HD-172-TEMP25-RTD-44 & -10.78 & 39.02 & -10.77 & 15.24 & 23.6 & 23.6 & 23.8 & 23.8 & 23.8 & 23.8 & 23.8 & 23.9 & 23.9 & 24.0 \\
\hline ESF-HD-172-TEMP25-RTD-45 & -10.99 & 39.02 & -10.99 & 15.54 & 23.7 & 23.7 & 23.8 & 23.8 & 23.8 & 23.8 & 23.8 & 23.9 & 23.9 & 24.0 \\
\hline ESF-HD-172-TEMP25-RTD-46 & -11.20 & 39.01 & -11.20 & 15.84 & 23.8 & 23.8 & 23.8 & 23.8 & 23.8 & 23.8 & 23.8 & 23.9 & 23.9 & 23.9 \\
\hline ESF-HD-172-TEMP25-RTD-47 & -11.41 & 39.00 & -11.41 & 16.14 & 23.8 & 23.7 & 23.8 & 23.8 & 23.7 & 23.8 & 23.8 & 23.8 & 23.9 & 23.9 \\
\hline ESF-HD-172-TEMP25-RTD-48 & -11.63 & 39.00 & -11.62 & 16.44 & 23.4 & 23.6 & 23.6 & 23.6 & 23.7 & 23.7 & 23.7 & 23.7 & 23.8 & 23.8 \\
\hline ESF-HD-172-TEMP25-RTD-49 & -11.84 & 38.99 & -11.84 & 16.74 & 23.8 & 23.8 & 23.8 & 23.8 & 23.8 & 23.8 & 23.8 & 23.9 & 23.9 & 23.9 \\
\hline ESF-HD-172-TEMP25-RTD-50 & -12.05 & 38.99 & -12.05 & 17.04 & 23.7 & 23.7 & 23.7 & 23.8 & 23.8 & 23.8 & 23.8 & 23.8 & 23.8 & 23.8 \\
\hline ESF-HD-172-TEMP25-RTD-51 & -12.26 & 38.98 & -12.26 & 17.34 & 23.6 & 23.7 & 23.7 & 23.8 & 23.8 & 23.8 & 23.8 & 23.8 & 23.8 & 23.8 \\
\hline ESF-HD-172-TEMP25-RTD-52 & -12.48 & 38.97 & -12.47 & 17.64 & 23.7 & 23.7 & 23.7 & 23.7 & 23.7 & 23.7 & 23.7 & 23.8 & 23.8 & 23.8 \\
\hline ESF-HD-172-TEMP25-RTD-53 & $\mid-12.69$ & 38.97 & -12.69 & 17.95 & 23.7 & 23.7 & 23.7 & 23.7 & 23.7 & 23.7 & 23.8 & 23.8 & 23.8 & 23.8 \\
\hline ESF-HD-172-TEMP25-RTD-54 & -12.90 & 38.96 & -12.90 & 18.24 & 23.7 & 23.8 & 23.8 & 23.8 & 23.8 & 23.8 & 23.8 & 23.8 & 23.8 & 23.8 \\
\hline ESF-HD-172-TEMP25-RTD-55 & -13.11 & 38.95 & -13.11 & 18.54 & 23.8 & 23.8 & 23.7 & 23.8 & 23.8 & 23.8 & 23.8 & 23.8 & 23.8 & 23.8 \\
\hline ESF-HD-172-TEMP25-RTD-56 & -13.33 & 38.95 & -13.32 & 18.84 & 23.7 & 23.5 & 23.6 & 23.7 & 23.7 & 23.7 & 23.7 & 23.7 & 23.7 & 23.8 \\
\hline ESF-HD-172-TEMP25-RTD-57 & -13.54 & 38.94 & -13.54 & 19.15 & 23.7 & 23.7 & 23.7 & 23.7 & 23.7 & 23.7 & 23.8 & 23.8 & 23.8 & 23.8 \\
\hline ESF-HD-172-TEMP25-RTD-58 & -13.75 & 38.94 & -13.75 & 19.45 & 23.6 & 23.5 & 23.6 & 23.7 & 23.7 & 23.7 & 23.7 & 23.7 & 23.7 & 23.8 \\
\hline ESF-HD-172-TEMP25-RTD-59 & -13.96 & 38.93 & -13.96 & 19.74 & 23.7 & 23.6 & 23.7 & 23.7 & 23.7 & 23.7 & 23.7 & 23.8 & 23.8 & 23.8 \\
\hline ESF-HD-172-TEMP25-RTD-60 & -14.17 & 38.92 & -14.17 & 20.04 & 23.7 & 23.5 & 23.7 & 23.7 & 23.7 & 23.7 & 23.7 & 23.7 & 23.7 & 23.8 \\
\hline ESF-HD-172-TEMP25-RTD-61 & -14.39 & 38.92 & -14.38 & 20.34 & 23.7 & 23.5 & 23.6 & 23.7 & 23.7 & 23.7 & 23.7 & 23.8 & 23.7 & 23.8 \\
\hline ESF-HD-172-TEMP25-RTD-62 & -14.60 & 38.91 & -14.60 & 20.65 & 23.8 & 23.7 & 23.6 & 23.8 & 23.7 & 23.7 & 23.8 & 23.8 & 23.8 & 23.8 \\
\hline ESF-HD-172-TEMP25-RTD-63 & -14.81 & 38.91 & -14.81 & 20.95 & 23.7 & 23.7 & 23.7 & 23.7 & 23.7 & 23.7 & 23.7 & 23.7 & 23.8 & 23.8 \\
\hline ESF-HD-172-TEMP25-RTD-64 & -15.02 & 38.90 & -15.02 & 21.24 & 23.7 & 23.7 & 23.7 & 23.7 & 23.7 & 23.7 & 23.7 & 23.7 & 23.7 & 23.7 \\
\hline ESF-HD-172-TEMP25-RTD-65 & -15.24 & 38.89 & -15.23 & 21.55 & 23.8 & 23.8 & 23.8 & 23.8 & 23.7 & 23.8 & 23.8 & 23.8 & 23.8 & 23.8 \\
\hline ESF-HD-172-TEMP25-RTD-66 & -15.45 & 38.89 & -15.45 & 21.85 & 23.7 & 23.7 & 23.7 & 23.7 & 23.7 & 23.7 & 23.7 & 23.7 & 23.7 & 23.7 \\
\hline ESF-HD-172-TEMP25-RTD-67 & -15.66 & 38.88 & -15.66 & 22.15 & 23.6 & 23.4 & 23.6 & 23.7 & 23.7 & 23.7 & 23.7 & 23.7 & 23.7 & 23.7 \\
\hline \multicolumn{15}{|l|}{ BH 173 Gage ID } \\
\hline ESF-HD-173-TEMP26-RTD-1 & 0.76 & 39.32 & -1.72 & 1.72 & 30.8 & & 51.2 & 58.3 & 64.3 & 69.5 & 74.1 & 78.5 & 82.1 & 84.4 \\
\hline ESF-HD-173-TEMP26-RTD-2 & 0.76 & 39.32 & -2.03 & 2.03 & 30.8 & 41.6 & 46.4 & 53.0 & 58.9 & 63.9 & 68.4 & 72.9 & 76.4 & 79.0 \\
\hline ESF-HD-173-TEMP26-RTD-3 & 0.76 & 39.32 & -2.33 & 2.33 & 30.7 & 28.4 & 42.3 & 48.4 & 54.1 & 58.9 & 63.3 & 67.6 & 71.2 & 73.7 \\
\hline ESF-HD-173-TEMP26-RTD-4 & 0.76 & 39.31 & -2.63 & 2.63 & 30.6 & 28.0 & 38.8 & 44.3 & 49.5 & 53.7 & 57.7 & 61.7 & 65.1 & 67.6 \\
\hline ESF-HD-173-TEMP26-RTD-5 & 0.76 & 39.31 & -2.93 & 2.93 & 30.0 & 27.6 & 36.0 & 40.9 & 45.8 & 49.8 & 53.6 & 57.4 & 60.7 & 63.1 \\
\hline ESF-HD-173-TEMP26-RTD-6 & 0.75 & 39.31 & -3.23 & 3.23 & 30.0 & 27.3 & 33.9 & 38.0 & 42.3 & 45.9 & 49.5 & 53.1 & 56.3 & 58.6 \\
\hline ESF-HD-173-TEMP26-RTD-7 & 0.75 & 39.30 & -3.53 & 3.53 & 28.7 & 26.7 & 31.8 & 35.4 & 39.1 & 42.6 & 45.9 & 49.3 & 52.3 & 54.6 \\
\hline ESF-HD-173-TEMP26-RTD-8 & 0.75 & 39.30 & -3.84 & 3.84 & 29.2 & 26.7 & 30.8 & 33.5 & 36.7 & 39.7 & 42.7 & 45.9 & 48.7 & 50.9 \\
\hline ESF-HD-173-TEMP26-RTD-9 & 0.75 & 39.30 & -4.14 & 4.14 & 28.8 & 26.3 & 29.8 & 31.9 & 34.6 & 37.3 & 39.9 & 42.9 & 45.5 & 47.6 \\
\hline ESF-HD-173-TEMP26-RTD-10 & 0.75 & 39.29 & -4.44 & 4.44 & 28.4 & 26.2 & 29.0 & 30.5 & 32.8 & 35.1 & 37.5 & 40.2 & 42.6 & 44.5 \\
\hline ESF-HD-173-TEMP26-RTD-11 & 0.75 & 39.29 & -4.74 & 4.74 & 28.0 & 25.5 & 28.3 & 29.5 & 31.3 & 33.3 & 35.4 & 37.8 & 40.0 & 41.8 \\
\hline ESF-HD-173-TEMP26-RTD-12 & 0.75 & 39.29 & -5.04 & 5.04 & 27.7 & 25.7 & 27.8 & 28.7 & 30.1 & 31.8 & 33.6 & 35.8 & 37.8 & 39.4 \\
\hline
\end{tabular}


First Quarter Report of Temperature Measurements During the Heating Phase of the Drift-Scale Test

\begin{tabular}{|c|c|c|c|c|c|c|c|c|c|c|c|c|c|c|}
\hline \multirow{5}{*}{\begin{tabular}{|l|} 
\\
ESF-HD-173-TEMP26-RTD-13 \\
\end{tabular}} & \multicolumn{14}{|c|}{ Temperature Data Obtained from the DST } \\
\hline & \multicolumn{14}{|c|}{ Days after startup } \\
\hline & \multicolumn{4}{|c|}{ As Built Locations (m) } & 0 & 7 & 17 & 27 & 38 & 48 & 58 & 69 & 79 & 87 \\
\hline & $\mathbf{x}$ & $\mathbf{y}$ & $\mathbf{z}$ & D & \multicolumn{10}{|c|}{$\left({ }^{\circ} \mathbf{C}\right)$} \\
\hline & 0.75 & 39.29 & -5.35 & 5.35 & 27.2 & 37.7 & 27.4 & 28.0 & 29.2 & 30.6 & 32.2 & 34.1 & 35.9 & 37.4 \\
\hline ESF-HD-173-TEMP26-RTD-14 & 0.75 & 39.28 & -5.65 & 5.65 & 27.0 & 25.5 & 27.0 & 27.5 & 28.2 & 29.4 & 30.8 & 32.5 & 34.0 & 35.4 \\
\hline ESF-HD-173-TEMP26-RTD-15 & 0.75 & 39.28 & -5.95 & 5.95 & 26.7 & 25.3 & 26.8 & 27.1 & 27.8 & 28.7 & 29.8 & 31.2 & 32.7 & 33.9 \\
\hline ESF-HD-173-TEMP26-RTD-16 & 0.75 & 39.28 & -6.25 & 6.25 & 26.4 & 25.1 & 26.5 & 26.7 & 27.2 & 27.9 & 28.9 & 30.1 & 31.3 & 32.4 \\
\hline ESF-HD-173-TEMP26-RTD-17 & 0.75 & 39.27 & -6.55 & 6.55 & 26.1 & 24.9 & 26.2 & 26.4 & 26.8 & 27.4 & 28.1 & 29.2 & 30.3 & 31.2 \\
\hline ESF-HD-173-TEMP26-RTD-18 & 0.75 & 39.27 & -6.86 & 6.86 & 25.7 & 24.9 & 25.7 & 26.1 & 26.3 & 26.8 & 27.4 & 28.3 & 29.3 & 30.1 \\
\hline ESF-HD-173-TEMP26-RTD-19 & 0.75 & 39.27 & -7.16 & 7.16 & 25.6 & 24.4 & 25.8 & 25.9 & 26.1 & 26.4 & 27.0 & 27.7 & 28.5 & 29.2 \\
\hline ESF-HD-173-TEMP26-RTD-20 & 0.75 & 39.26 & -7.46 & 7.46 & 25.4 & 24.5 & 25.6 & 25.7 & 25.8 & 26.1 & 26.5 & 27.1 & 27.8 & 28.4 \\
\hline ESF-HD-173-TEMP26-RTD-21 & 0.75 & 39.26 & -7.76 & 7.76 & 25.2 & 24.5 & 25.4 & 25.5 & 25.6 & 25.8 & 26.1 & 26.6 & 27.2 & 27.7 \\
\hline ESF-HD-173-TEMP26-RTD-22 & 0.74 & 39.26 & -8.06 & 8.06 & 25.1 & 24.4 & 25.2 & 25.3 & 25.4 & 25.6 & 25.8 & 26.2 & 26.7 & 27.2 \\
\hline ESF-HD-173-TEMP26-RTD-23 & 0.74 & 39.25 & -8.36 & 8.36 & 24.9 & 24.3 & 25.0 & 25.1 & 25.1 & 25.3 & 25.5 & 25.8 & 26.2 & 26.6 \\
\hline ESF-HD-173-TEMP26-RTD-24 & 0.74 & 39.25 & -8.67 & 8.67 & 24.8 & 34.8 & 24.9 & 25.0 & 25.1 & 25.2 & 25.3 & 25.6 & 25.9 & 26.3 \\
\hline ESF-HD-173-TEMP26-RTD-25 & 0.74 & 39.25 & -8.97 & 8.97 & 24.7 & 24.3 & 21.6 & 24.6 & 24.7 & 24.9 & 25.1 & 25.4 & 25.6 & 25.9 \\
\hline ESF-HD-173-TEMP26-RTD-26 & 0.74 & 39.24 & -9.27 & 9.27 & 24.4 & 24.2 & 24.5 & 24.6 & 24.7 & 24.7 & 24.8 & 25.0 & 25.2 & 25.5 \\
\hline ESF-HD-173-TEMP26-RTD-27 & 0.74 & 39.24 & -9.57 & 9.57 & 24.5 & 24.2 & 24.6 & 24.6 & 24.7 & 24.7 & 24.8 & 25.0 & 25.2 & 25.3 \\
\hline ESF-HD-173-TEMP26-RTD-28 & 0.74 & 39.24 & -9.87 & 9.87 & 24.4 & 24.1 & 24.4 & 24.5 & 24.6 & 24.6 & 24.7 & 24.8 & 25.0 & 25.1 \\
\hline ESF-HD-173-TEMP26-RTD-29 & 0.74 & 39.24 & -10.18 & 10.18 & 24.3 & 24.1 & 24.4 & 24.4 & 24.4 & 24.5 & 24.6 & 24.7 & 24.8 & 24.9 \\
\hline ESF-HD-173-TEMP26-RTD-30 & 0.74 & 39.23 & -10.48 & 10.48 & 24.2 & 24.1 & 24.3 & 24.3 & 24.4 & 24.4 & 24.5 & 24.6 & 24.7 & 24.8 \\
\hline ESF-HD-173-TEMP26-RTD-31 & 0.74 & 39.23 & -10.78 & 10.78 & 24.2 & 24.0 & 24.2 & 24.3 & 24.3 & 24.4 & 24.4 & 24.5 & 24.6 & 24.7 \\
\hline ESF-HD-173-TEMP26-RTD-32 & 0.74 & 39.23 & -11.08 & 11.08 & 24.2 & 24.0 & 24.3 & 24.3 & 24.4 & 24.4 & 24.5 & 24.5 & 24.6 & 24.7 \\
\hline ESF-HD-173-TEMP26-RTD-33 & 0.74 & 39.22 & -11.38 & 11.38 & 24.1 & 23.8 & 24.2 & 24.2 & 24.2 & 24.3 & 24.3 & 24.4 & 24.4 & 24.5 \\
\hline ESF-HD-173-TEMP26-RTD-34 & 0.74 & 39.22 & -11.68 & 11.68 & 24.1 & 24.0 & 24.1 & 24.2 & 24.2 & 24.2 & 24.3 & 24.3 & 24.4 & 24.4 \\
\hline ESF-HD-173-TEMP26-RTD-35 & 0.74 & 39.22 & -11.99 & 11.99 & 24.0 & 32.7 & 24.1 & 24.1 & 24.1 & 24.2 & 24.2 & 24.2 & 24.3 & 24.3 \\
\hline ESF-HD-173-TEMP26-RTD-36 & 0.74 & 39.21 & -12.29 & 12.29 & 24.0 & 23.9 & 24.0 & 24.1 & 24.1 & 24.1 & 24.2 & 24.2 & 24.2 & 24.3 \\
\hline ESF-HD-173-TEMP26-RTD-37 & 0.74 & 39.21 & -12.59 & 12.59 & 24.0 & 23.9 & 24.0 & 24.0 & 24.1 & 24.1 & 24.1 & 24.1 & 24.2 & 24.2 \\
\hline ESF-HD-173-TEMP26-RTD-38 & 0.74 & 39.21 & -12.89 & 12.89 & 23.9 & 8.9 & 24.0 & 24.0 & 24.0 & 24.0 & 24.0 & 24.1 & 24.1 & 24.1 \\
\hline ESF-HD-173-TEMP26-RTD-39 & 0.73 & 39.20 & -13.19 & 13.19 & 23.9 & 23.9 & 24.0 & 24.0 & 24.0 & 24.0 & 24.1 & 24.1 & 24.1 & 24.1 \\
\hline ESF-HD-173-TEMP26-RTD-40 & 0.73 & 39.20 & -13.50 & 13.50 & 23.8 & 23.9 & 23.9 & 23.9 & 24.0 & 24.0 & 24.0 & 24.0 & 24.0 & 24.1 \\
\hline ESF-HD-173-TEMP26-RTD-41 & 0.73 & 39.20 & -13.80 & 13.80 & 23.9 & 23.9 & 23.9 & 23.9 & 24.0 & 24.0 & 24.0 & 24.0 & 24.0 & 24.1 \\
\hline ESF-HD-173-TEMP26-RTD-42 & 0.73 & 39.19 & -14.10 & 14.10 & 23.8 & 23.9 & 23.9 & 23.9 & 24.0 & 23.9 & 24.0 & 24.0 & 24.0 & 24.0 \\
\hline ESF-HD-173-TEMP26-RTD-43 & 0.73 & 39.19 & -14.40 & 14.40 & 23.9 & 23.8 & 23.9 & 23.9 & 23.9 & 23.9 & 23.9 & 24.0 & 24.0 & 24.0 \\
\hline ESF-HD-173-TEMP26-RTD-44 & 0.73 & 39.19 & -14.70 & 14.70 & 23.9 & 23.8 & 23.9 & 23.9 & 23.9 & 23.9 & 23.9 & 24.0 & 24.0 & 24.0 \\
\hline ESF-HD-173-TEMP26-RTD-45 & 0.73 & 39.19 & -15.01 & 15.01 & 23.9 & 23.9 & 23.9 & 23.9 & 23.9 & 23.9 & 23.9 & 23.9 & 24.0 & 24.0 \\
\hline ESF-HD-173-TEMP26-RTD-46 & 0.73 & 39.18 & -15.31 & 15.31 & 23.9 & 31.1 & 23.9 & 23.9 & 23.9 & 23.9 & 23.9 & 23.9 & 23.9 & 23.9 \\
\hline ESF-HD-173-TEMP26-RTD-47 & 0.73 & 39.18 & -15.61 & 15.61 & 23.7 & 23.8 & 23.8 & 23.9 & 23.9 & 23.9 & 23.9 & 23.9 & 23.9 & 23.9 \\
\hline ESF-HD-173-TEMP26-RTD-48 & 0.73 & 39.18 & -15.91 & 15.91 & 23.8 & 23.8 & 23.8 & 23.9 & 23.9 & 23.9 & 23.9 & 23.9 & 23.9 & 23.9 \\
\hline ESF-HD-173-TEMP26-RTD-49 & 0.73 & 39.17 & -16.21 & 16.21 & 23.8 & 23.6 & 23.9 & 23.9 & 23.9 & 23.9 & 23.9 & 23.9 & 23.9 & 23.9 \\
\hline
\end{tabular}




\begin{tabular}{|c|c|c|c|c|c|c|c|c|c|c|c|c|c|c|}
\hline & \multicolumn{14}{|c|}{ Temperature Data Obtained from the DST } \\
\hline & \multicolumn{14}{|c|}{ Days after startup } \\
\hline & \multicolumn{4}{|c|}{ As Built Locations (m) } & 0 & 7 & 17 & 27 & 38 & 48 & 58 & 69 & 79 & 87 \\
\hline & $\mathbf{x}$ & $\mathbf{y}$ & $\mathbf{z}$ & D & \multicolumn{10}{|c|}{$\left({ }^{\circ} \mathbf{C}\right)$} \\
\hline ESF-HD-173-TEMP26-RTD-50 & 0.73 & 39.17 & -16.51 & 16.51 & 23.8 & 23.9 & 23.8 & 23.9 & 23.9 & 23.9 & 23.9 & 23.9 & 23.9 & 23.9 \\
\hline ESF-HD-173-TEMP26-RTD-51 & 0.73 & 39.17 & -16.82 & 16.82 & 23.8 & 23.8 & 23.8 & 23.8 & 23.8 & 23.9 & 23.9 & 23.9 & 23.9 & 23.9 \\
\hline ESF-HD-173-TEMP26-RTD-52 & 0.73 & 39.16 & -17.12 & 17.12 & 23.6 & 23.9 & 23.8 & 23.8 & 23.8 & 23.9 & 23.9 & 23.9 & 23.9 & 23.9 \\
\hline ESF-HD-173-TEMP26-RTD-53 & 0.73 & 39.16 & -17.42 & 17.42 & 23.9 & 23.8 & 23.9 & 23.9 & 23.9 & 23.9 & 23.9 & 23.9 & 23.9 & 23.9 \\
\hline ESF-HD-173-TEMP26-RTD-54 & 0.73 & 39.16 & -17.72 & 17.72 & 23.8 & 23.8 & 23.8 & 23.8 & 23.8 & 23.8 & 23.8 & 23.8 & 23.8 & 23.8 \\
\hline ESF-HD-173-TEMP26-RTD-55 & 0.72 & 39.15 & -18.02 & 18.02 & 23.9 & 23.9 & 23.9 & 23.9 & 23.9 & 23.9 & 23.9 & 23.9 & 23.9 & 23.9 \\
\hline ESF-HD-173-TEMP26-RTD-56 & 0.72 & 39.15 & -18.33 & 18.33 & 23.8 & 23.7 & 23.8 & 23.8 & 23.8 & 23.8 & 23.8 & 23.9 & 23.9 & 23.9 \\
\hline ESF-HD-173-TEMP26-RTD-57 & 0.72 & 39.15 & -18.63 & 18.63 & 23.8 & 30.4 & 23.8 & 23.8 & 23.8 & 23.8 & 23.8 & 23.8 & 23.8 & 23.8 \\
\hline ESF-HD-173-TEMP26-RTD-58 & 0.72 & 39.14 & -18.93 & 18.93 & 23.8 & 23.8 & 23.9 & 23.9 & 23.9 & 23.9 & 23.9 & 23.9 & 23.9 & 23.9 \\
\hline ESF-HD-173-TEMP26-RTD-59 & 0.72 & 39.14 & -19.23 & 19.23 & 23.6 & 23.8 & 23.8 & 23.8 & 23.8 & 23.8 & 23.8 & 23.8 & 23.8 & 23.8 \\
\hline ESF-HD-173-TEMP26-RTD-60 & 0.72 & 39.14 & -19.53 & 19.53 & 23.8 & 23.8 & 23.8 & 23.8 & 23.8 & 23.8 & 23.8 & 23.8 & 23.8 & 23.8 \\
\hline ESF-HD-173-TEMP26-RTD-61 & 0.72 & 39.14 & -19.84 & 19.84 & 23.8 & 23.9 & 23.8 & 23.8 & 23.8 & 23.8 & 23.8 & 23.8 & 23.8 & 23.8 \\
\hline ESF-HD-173-TEMP26-RTD-62 & 0.72 & 39.13 & -20.14 & 20.14 & 23.8 & 23.8 & 23.8 & 23.9 & 23.8 & 23.9 & 23.9 & 23.9 & 23.9 & 23.9 \\
\hline ESF-HD-173-TEMP26-RTD-63 & 0.72 & 39.13 & -20.44 & 20.44 & 23.9 & 23.8 & 23.7 & 23.9 & 23.9 & 23.9 & 23.9 & 23.9 & 23.9 & 23.9 \\
\hline ESF-HD-173-TEMP26-RTD-64 & 0.72 & 39.13 & -20.74 & 20.74 & 23.8 & 23.8 & 23.8 & 23.8 & 23.8 & 23.8 & 23.8 & 23.8 & 23.8 & 23.8 \\
\hline ESF-HD-173-TEMP26-RTD-65 & 0.72 & 39.12 & -21.04 & 21.04 & 23.9 & 23.9 & 23.8 & 23.8 & 23.8 & 23.8 & 23.8 & 23.8 & 23.8 & 23.8 \\
\hline ESF-HD-173-TEMP26-RTD-66 & 0.72 & 39.12 & -21.34 & 21.34 & 23.9 & 28.7 & 23.8 & 23.8 & 23.8 & 23.8 & 23.8 & 23.8 & 23.8 & 23.8 \\
\hline ESF-HD-173-TEMP26-RTD-67 & 0.72 & 39.12 & -21.65 & 21.65 & 23.9 & 29.2 & 23.9 & 23.9 & 23.9 & 23.9 & 23.9 & 23.9 & 23.9 & 23.9 \\
\hline \multicolumn{15}{|l|}{ BH 174 Gage ID } \\
\hline ESF-HD-174-TEMP27-RTD-1 & 1.52 & 39.31 & -1.53 & 2.16 & 30.6 & 45.6 & 55.2 & 62.5 & 68.7 & 73.9 & 78.6 & 83.2 & 86.8 & 89.3 \\
\hline ESF-HD-174-TEMP27-RTD-2 & 1.75 & 39.30 & -1.74 & 2.47 & 30.6 & 39.9 & 48.5 & 55.6 & 61.6 & 66.7 & 71.3 & 75.7 & 79.5 & 81.9 \\
\hline ESF-HD-174-TEMP27-RTD-3 & 1.97 & 39.30 & -1.95 & 2.77 & 30.5 & 36.5 & 44.4 & 50.9 & 56.8 & 61.7 & 66.2 & 70.6 & 74.3 & 76.9 \\
\hline ESF-HD-174-TEMP27-RTD-4 & 2.20 & 39.29 & -2.16 & 3.08 & 30.3 & 34.0 & 40.8 & 46.8 & 52.5 & 57.3 & 61.7 & 66.1 & 69.8 & 72.4 \\
\hline ESF-HD-174-TEMP27-RTD-5 & 2.42 & 39.29 & -2.37 & 3.39 & 30.0 & 31.9 & 37.3 & 42.7 & 48.1 & 52.8 & 57.1 & 61.4 & 65.1 & 67.8 \\
\hline ESF-HD-174-TEMP27-RTD-6 & 2.65 & 39.28 & -2.58 & 3.70 & 29.7 & 30.6 & 35.0 & 39.8 & 44.9 & 49.4 & 53.6 & 57.9 & 61.5 & 64.2 \\
\hline ESF-HD-174-TEMP27-RTD-7 & 2.87 & 39.27 & -2.79 & 4.00 & 29.0 & 29.4 & 32.9 & 37.2 & 41.9 & 46.1 & 50.2 & 54.4 & 57.9 & 60.6 \\
\hline ESF-HD-174-TEMP27-RTD-8 & 3.10 & 39.27 & -3.00 & 4.31 & 29.0 & 29.1 & 31.5 & 35.1 & 39.5 & 43.4 & 47.3 & 51.3 & 54.8 & 57.5 \\
\hline ESF-HD-174-TEMP27-RTD-9 & 3.32 & 39.26 & -3.21 & 4.62 & 28.6 & 28.6 & 30.3 & 33.4 & 37.4 & 41.1 & 44.7 & 48.6 & 52.0 & 54.6 \\
\hline ESF-HD-174-TEMP27-RTD-10 & 3.54 & 39.26 & -3.42 & 4.92 & 28.2 & 28.2 & 29.4 & 32.0 & 35.5 & 38.9 & 42.4 & 46.1 & 49.4 & 51.9 \\
\hline ESF-HD-174-TEMP27-RTD-11 & 3.77 & 39.25 & -3.63 & 5.23 & 27.9 & 27.9 & 28.7 & 30.8 & 33.9 & 37.0 & 40.3 & 43.8 & 47.0 & 49.4 \\
\hline ESF-HD-174-TEMP27-RTD-12 & 3.99 & 39.25 & -3.84 & 5.54 & 27.5 & 27.5 & 28.1 & 29.8 & 32.6 & 35.5 & 38.5 & 41.8 & 44.9 & 47.2 \\
\hline ESF-HD-174-TEMP27-RTD-13 & 4.22 & 39.24 & -4.05 & 5.85 & 27.2 & 27.2 & 27.6 & 29.0 & 31.3 & 33.9 & 36.7 & 39.9 & 42.7 & 45.0 \\
\hline ESF-HD-174-TEMP27-RTD-14 & 4.44 & 39.24 & -4.26 & 6.15 & 26.9 & 26.9 & 27.2 & 28.3 & 30.3 & 32.7 & 35.2 & 38.2 & 40.9 & 43.1 \\
\hline ESF-HD-174-TEMP27-RTD-15 & 4.67 & 39.23 & -4.47 & 6.46 & 26.6 & 26.6 & 26.8 & 27.7 & 29.4 & 31.5 & 33.8 & 36.5 & 39.1 & 41.2 \\
\hline ESF-HD-174-TEMP27-RTD-16 & 4.89 & 39.23 & -4.68 & 6.77 & 26.3 & 26.3 & 26.5 & 27.2 & 28.7 & 30.6 & 32.8 & 35.3 & 37.8 & 39.8 \\
\hline ESF-HD-174-TEMP27-RTD-17 & 5.12 & 39.22 & -4.89 & 7.08 & 26.0 & 26.1 & 26.2 & 26.8 & 28.0 & 29.6 & 31.5 & 33.9 & 36.1 & 38.0 \\
\hline ESF-HD-174-TEMP27-RTD-18 & 5.34 & 39.21 & -5.10 & 7.38 & 25.8 & 25.9 & 26.0 & 26.4 & 27.5 & 28.9 & 30.6 & 32.8 & 34.9 & 36.6 \\
\hline
\end{tabular}


First Quarter Report of Temperature Measurements During the Heating Phase of the Drift-Scale Test

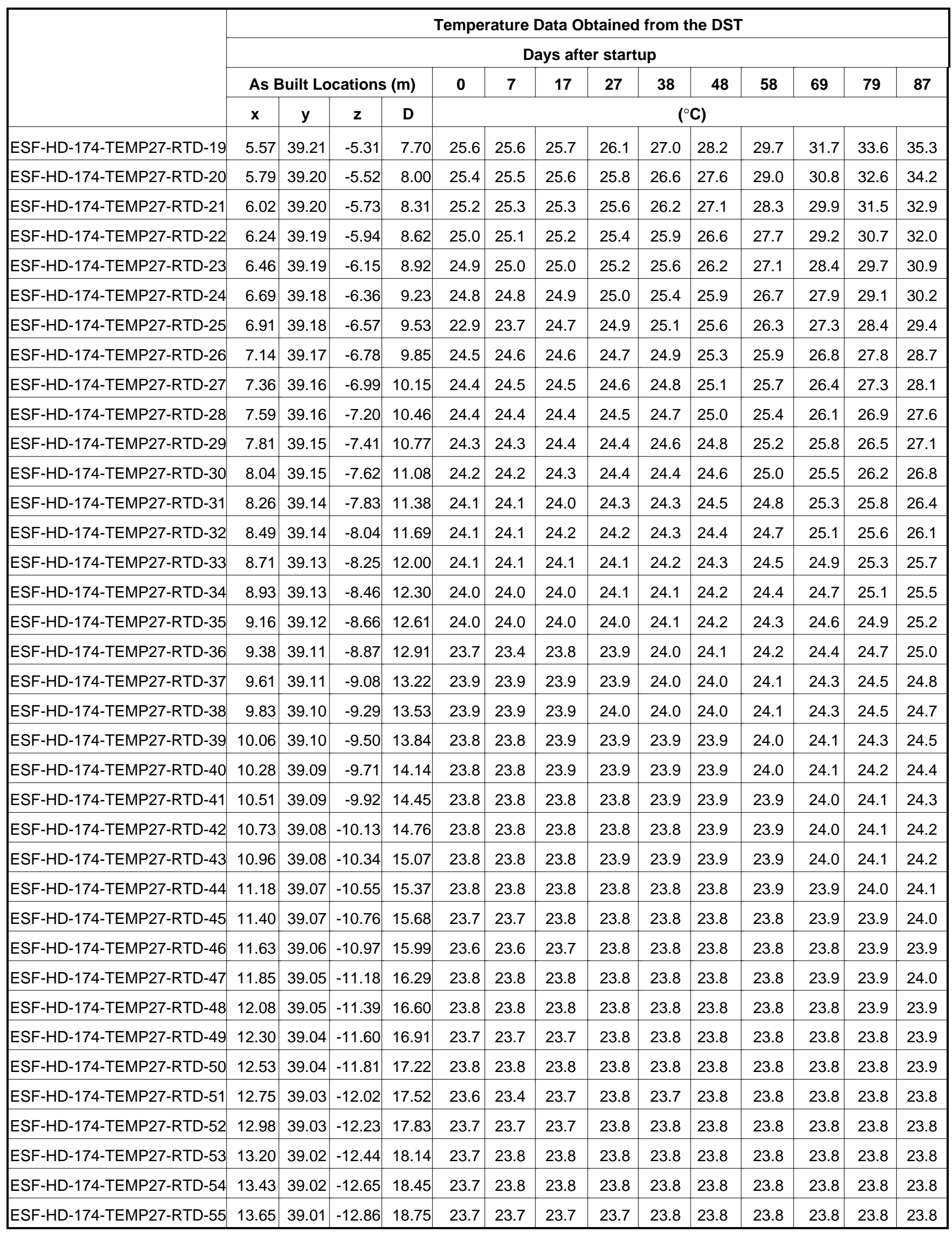




\begin{tabular}{|c|c|c|c|c|c|c|c|c|c|c|c|c|c|c|}
\hline & \multicolumn{14}{|c|}{ Temperature Data Obtained from the DST } \\
\hline & \multicolumn{14}{|c|}{ Days after startup } \\
\hline & \multicolumn{4}{|c|}{ As Built Locations (m) } & 0 & 7 & 17 & 27 & 38 & 48 & 58 & 69 & 79 & 87 \\
\hline & $\mathbf{x}$ & $\mathbf{y}$ & $\mathbf{z}$ & D & \multicolumn{10}{|c|}{$\left({ }^{\circ} \mathbf{C}\right)$} \\
\hline ESF-HD-174-TEMP27-RTD-56 & 13.87 & 39.00 & -13.07 & 19.06 & 23.7 & 23.7 & 23.7 & 23.7 & 22.9 & 21.3 & 20.8 & 23.5 & 23.1 & 23.7 \\
\hline ESF-HD-174-TEMP27-RTD-57 & 14.10 & 39.00 & -13.28 & 19.37 & 23.5 & 23.3 & 23.4 & 23.7 & 23.6 & 23.7 & 23.3 & 23.7 & 23.5 & 23.8 \\
\hline ESF-HD-174-TEMP27-RTD-58 & 14.32 & 38.99 & -13.49 & 19.67 & 23.8 & 23.8 & 23.8 & 23.8 & 23.8 & 23.8 & 23.8 & 23.8 & 23.8 & 23.8 \\
\hline ESF-HD-174-TEMP27-RTD-59 & 14.55 & 38.99 & -13.70 & 19.99 & 23.7 & 23.7 & 23.7 & 23.7 & 23.7 & 23.7 & 23.7 & 23.7 & 23.7 & 23.8 \\
\hline ESF-HD-174-TEMP27-RTD-60 & 14.77 & 38.98 & -13.91 & 20.29 & 23.7 & 23.7 & 23.7 & 23.7 & 23.7 & 23.7 & 23.7 & 23.7 & 23.7 & 23.8 \\
\hline ESF-HD-174-TEMP27-RTD-61 & 15.00 & 38.98 & -14.12 & 20.60 & 23.7 & 23.7 & 23.7 & 23.7 & 23.7 & 23.7 & 23.7 & 23.8 & 23.8 & 23.8 \\
\hline ESF-HD-174-TEMP27-RTD-62 & 15.22 & 38.97 & -14.33 & 20.90 & 23.8 & 23.7 & 23.7 & 23.7 & 23.7 & 23.7 & 23.7 & 23.8 & 23.8 & 23.8 \\
\hline ESF-HD-174-TEMP27-RTD-63 & 15.45 & 38.97 & -14.54 & 21.22 & 23.8 & 23.8 & 23.8 & 23.8 & 23.8 & 23.8 & 23.8 & 23.8 & 23.8 & 23.8 \\
\hline ESF-HD-174-TEMP27-RTD-64 & 15.67 & 38.96 & -14.75 & 21.52 & 23.8 & 23.7 & 23.7 & 23.7 & 23.7 & 23.7 & 23.7 & 23.7 & 23.7 & 23.7 \\
\hline ESF-HD-174-TEMP27-RTD-65 & 15.90 & 38.96 & -14.96 & 21.83 & 23.8 & 23.8 & 23.8 & 23.8 & 23.8 & 23.8 & 23.8 & 23.8 & 23.8 & 23.8 \\
\hline ESF-HD-174-TEMP27-RTD-66 & 16.12 & 38.95 & -15.17 & 22.14 & 23.8 & 23.8 & 23.8 & 23.8 & 23.8 & 23.8 & 23.8 & 23.8 & 23.8 & 23.8 \\
\hline ESF-HD-174-TEMP27-RTD-67 & 16.34 & 38.94 & -15.38 & 22.44 & 23.7 & 21.1 & 22.0 & 23.1 & 22.5 & 22.6 & 22.6 & 23.3 & 22.4 & 23.4 \\
\hline \multicolumn{15}{|l|}{ BH 175 Gage ID } \\
\hline ESF-HD-175-TEMP28-RTD-1 & 1.94 & 39.32 & 1.90 & 2.72 & 30.4 & 52.1 & 61.9 & 68.9 & 75.2 & 80.3 & 84.8 & 88.9 & 92.5 & 94.6 \\
\hline ESF-HD-175-TEMP28-RTD-2 & 2.15 & 39.32 & 2.12 & 3.02 & 30.2 & 43.9 & 53.5 & 60.5 & 66.8 & 72.0 & 76.4 & 80.7 & 84.1 & 86.5 \\
\hline ESF-HD-175-TEMP28-RTD-3 & 2.36 & 39.31 & 2.33 & 3.32 & 30.3 & 38.6 & 47.4 & 54.2 & 60.4 & 65.5 & 70.0 & 74.3 & 77.8 & 80.3 \\
\hline ESF-HD-175-TEMP28-RTD-4 & 2.56 & 39.31 & 2.55 & 3.61 & 30.1 & 35.0 & 42.9 & 49.3 & 55.3 & 60.3 & 64.8 & 69.2 & 72.8 & 75.4 \\
\hline ESF-HD-175-TEMP28-RTD-5 & 2.77 & 39.31 & 2.76 & 3.91 & 29.9 & 32.7 & 39.5 & 45.4 & 51.2 & 56.0 & 60.4 & 64.8 & 68.4 & 71.1 \\
\hline ESF-HD-175-TEMP28-RTD-6 & 2.98 & 39.31 & 2.98 & 4.21 & 29.7 & 31.3 & 36.9 & 42.3 & 47.8 & 52.5 & 56.8 & 61.1 & 64.8 & 67.4 \\
\hline ESF-HD-175-TEMP28-RTD-7 & 3.19 & 39.31 & 3.19 & 4.51 & 29.3 & 30.2 & 34.5 & 39.3 & 44.3 & 48.8 & 52.9 & 57.1 & 60.7 & 63.4 \\
\hline ESF-HD-175-TEMP28-RTD-8 & 3.40 & 39.30 & 3.41 & 4.82 & 29.0 & 29.4 & 32.6 & 36.7 & 41.3 & 45.4 & 49.4 & 53.4 & 57.0 & 59.6 \\
\hline ESF-HD-175-TEMP28-RTD-9 & 3.61 & 39.30 & 3.62 & 5.11 & 28.5 & 28.5 & 31.0 & 34.4 & 38.5 & 42.3 & 46.1 & 50.0 & 53.4 & 56.1 \\
\hline ESF-HD-175-TEMP28-RTD-10 & 3.82 & 39.30 & 3.84 & 5.42 & 28.2 & 28.3 & 29.8 & 32.6 & 36.2 & 39.7 & 43.2 & 46.9 & 50.2 & 52.8 \\
\hline ESF-HD-175-TEMP28-RTD-11 & 4.03 & 39.30 & 4.06 & 5.72 & 27.9 & 27.8 & 28.9 & 31.1 & 34.2 & 37.3 & 40.6 & 44.1 & 47.2 & 49.7 \\
\hline ESF-HD-175-TEMP28-RTD-12 & 4.24 & 39.30 & 4.27 & 6.02 & 27.5 & 27.5 & 28.1 & 29.9 & 32.5 & 35.3 & 38.3 & 41.5 & 44.5 & 46.8 \\
\hline ESF-HD-175-TEMP28-RTD-13 & 4.45 & 39.29 & 4.49 & 6.32 & 27.2 & 27.1 & 27.6 & 28.9 & 31.1 & 33.6 & 36.2 & 39.2 & 42.0 & 44.2 \\
\hline ESF-HD-175-TEMP28-RTD-14 & 4.66 & 39.29 & 4.70 & 6.62 & 26.8 & 26.8 & 27.1 & 28.1 & 30.0 & 32.1 & 34.5 & 37.3 & 39.9 & 42.0 \\
\hline ESF-HD-175-TEMP28-RTD-15 & 4.87 & 39.29 & 4.92 & 6.92 & 26.5 & 26.5 & 26.7 & 27.5 & 29.0 & 30.8 & 33.0 & 35.5 & 37.9 & 39.9 \\
\hline ESF-HD-175-TEMP28-RTD-16 & 5.08 & 39.29 & 5.13 & 7.22 & 26.2 & 26.3 & 26.4 & 27.0 & 28.2 & 29.9 & 31.8 & 34.1 & 36.3 & 38.1 \\
\hline ESF-HD-175-TEMP28-RTD-17 & 5.29 & 39.29 & 5.35 & 7.52 & 26.0 & 26.0 & 26.1 & 26.5 & 27.6 & 28.9 & 30.6 & 32.7 & 34.7 & 36.4 \\
\hline ESF-HD-175-TEMP28-RTD-18 & 5.50 & 39.28 & 5.56 & 7.82 & 25.4 & 25.5 & 25.7 & 26.1 & 26.8 & 27.9 & 29.5 & 31.4 & 33.2 & 34.8 \\
\hline ESF-HD-175-TEMP28-RTD-19 & 5.71 & 39.28 & 5.78 & 8.12 & 25.5 & 25.5 & 25.6 & 25.8 & 26.4 & 27.4 & 28.7 & 30.3 & 32.0 & 33.4 \\
\hline ESF-HD-175-TEMP28-RTD-20 & 5.92 & 39.28 & 5.99 & 8.42 & 25.3 & 25.3 & 25.4 & 25.6 & 26.1 & 26.9 & 28.0 & 29.5 & 31.0 & 32.3 \\
\hline ESF-HD-175-TEMP28-RTD-21 & 6.13 & 39.28 & 6.21 & 8.73 & 25.1 & 25.2 & 25.2 & 25.4 & 25.8 & 26.5 & 27.4 & 28.7 & 30.1 & 31.3 \\
\hline ESF-HD-175-TEMP28-RTD-22 & 6.34 & 39.27 & 6.43 & 9.03 & 24.9 & 25.0 & 25.1 & 25.2 & 25.5 & 26.1 & 26.9 & 28.0 & 29.2 & 30.3 \\
\hline ESF-HD-175-TEMP28-RTD-23 & 6.55 & 39.27 & 6.64 & 9.33 & 23.4 & 23.6 & 23.8 & 24.2 & 24.6 & 25.2 & 25.9 & 27.1 & 28.3 & 29.3 \\
\hline ESF-HD-175-TEMP28-RTD-24 & 6.76 & 39.27 & 6.86 & 9.63 & 24.6 & 24.7 & 24.8 & 24.8 & 25.1 & 25.4 & 26.0 & 26.9 & 27.9 & 28.8 \\
\hline
\end{tabular}


First Quarter Report of Temperature Measurements During the Heating Phase of the Drift-Scale Test

\begin{tabular}{|c|c|c|c|c|c|c|c|c|c|c|c|c|c|c|}
\hline \multirow{5}{*}{\begin{tabular}{|l|} 
\\
ESF-HD-175-TEMP28-RTD-25 \\
\end{tabular}} & \multicolumn{14}{|c|}{ Temperature Data Obtained from the DST } \\
\hline & \multicolumn{14}{|c|}{ Days after startup } \\
\hline & \multicolumn{4}{|c|}{ As Built Locations (m) } & 0 & 7 & 17 & 27 & 38 & 48 & 58 & 69 & 79 & 87 \\
\hline & \multirow{2}{*}{\begin{tabular}{c|}
$\mathbf{x}$ \\
6.97 \\
\end{tabular}} & \multirow{2}{*}{\begin{tabular}{|c|}
$\mathbf{y}$ \\
39.27 \\
\end{tabular}} & \multirow{2}{*}{$\begin{array}{l}\mathbf{z} \\
7.07 \\
\end{array}$} & \multirow{2}{*}{\begin{tabular}{l|}
$\mathbf{D}$ \\
9.93
\end{tabular}} & \multicolumn{10}{|c|}{$\left({ }^{\circ} \mathbf{C}\right)$} \\
\hline & & & & & 24.5 & 24.6 & 24.6 & 24.7 & 24.9 & 25.2 & 25.7 & 26.5 & 27.3 & 28.1 \\
\hline ESF-HD-175-TEMP28-RTD-26 & 7.17 & 39.27 & 7.29 & 10.23 & 24.4 & 24.4 & 24.5 & 24.5 & 24.7 & 24.9 & 25.3 & 26.0 & 26.8 & 27.5 \\
\hline ESF-HD-175-TEMP28-RTD-27 & 7.38 & 39.26 & 7.50 & 10.52 & 24.3 & 24.3 & 24.4 & 24.4 & 24.6 & 24.8 & 25.1 & 25.7 & 26.4 & 27.0 \\
\hline ESF-HD-175-TEMP28-RTD-28 & 7.59 & 39.26 & 7.72 & 10.83 & 24.2 & 24.2 & 24.3 & 24.3 & 24.4 & 24.6 & 24.9 & 25.4 & 26.0 & 26.6 \\
\hline ESF-HD-175-TEMP28-RTD-29 & 7.80 & 39.26 & 7.93 & 11.12 & 24.1 & 24.1 & 24.2 & 24.3 & 24.3 & 24.5 & 24.7 & 25.2 & 25.7 & 26.2 \\
\hline ESF-HD-175-TEMP28-RTD-30 & 8.01 & 39.26 & 8.15 & 11.43 & 24.0 & 24.1 & 24.1 & 24.2 & 24.2 & 24.4 & 24.6 & 24.9 & 25.4 & 25.8 \\
\hline ESF-HD-175-TEMP28-RTD-31 & 8.22 & 39.26 & 8.37 & 11.73 & 24.0 & 24.1 & 24.1 & 24.2 & 24.2 & 24.4 & 24.6 & 24.9 & 25.4 & 25.8 \\
\hline ESF-HD-175-TEMP28-RTD-32 & 8.43 & 39.25 & 8.58 & 12.03 & 23.9 & 23.9 & 24.0 & 24.0 & 24.1 & 24.1 & 24.3 & 24.5 & 24.9 & 25.2 \\
\hline ESF-HD-175-TEMP28-RTD-33 & 8.64 & 39.25 & 8.80 & 12.33 & 23.8 & 23.9 & 23.9 & 24.0 & 24.0 & 24.1 & 24.2 & 24.4 & 24.7 & 25.0 \\
\hline ESF-HD-175-TEMP28-RTD-34 & 8.85 & 39.25 & 9.01 & 12.63 & 23.8 & 23.9 & 23.9 & 24.0 & 24.0 & 24.1 & 24.2 & 24.4 & 24.7 & 25.0 \\
\hline ESF-HD-175-TEMP28-RTD-35 & 9.06 & 39.25 & 9.23 & 12.93 & 23.7 & 23.7 & 23.8 & 23.8 & 23.8 & 23.9 & 24.0 & 24.1 & 24.4 & 24.6 \\
\hline ESF-HD-175-TEMP28-RTD-36 & 9.27 & 39.24 & 9.44 & 13.23 & 23.6 & 23.1 & 23.6 & 23.7 & 23.8 & 23.8 & 23.9 & 24.0 & 24.2 & 24.4 \\
\hline ESF-HD-175-TEMP28-RTD-37 & 9.48 & 39.24 & 9.66 & 13.54 & 23.6 & 23.7 & 23.7 & 23.7 & 23.8 & 23.8 & 23.9 & 24.0 & 24.1 & 24.3 \\
\hline ESF-HD-175-TEMP28-RTD-38 & 9.69 & 39.24 & 9.87 & 13.83 & 23.6 & 23.6 & 23.6 & 23.7 & 23.7 & 23.7 & 23.8 & 23.9 & 24.0 & 24.1 \\
\hline ESF-HD-175-TEMP28-RTD-39 & 9.90 & 39.24 & 10.09 & 14.14 & 23.3 & 23.6 & 23.6 & 23.6 & 23.7 & 23.7 & 23.7 & 23.8 & 23.9 & 24.1 \\
\hline ESF-HD-175-TEMP28-RTD-40 & 10.11 & 39.24 & 10.30 & 14.43 & 23.6 & 23.6 & 23.6 & 23.6 & 23.6 & 23.7 & 23.7 & 23.8 & 23.9 & 24.0 \\
\hline ESF-HD-175-TEMP28-RTD-41 & 10.32 & 39.23 & 10.52 & 14.74 & 23.7 & 22.3 & 23.4 & 23.6 & 23.6 & 23.7 & 23.7 & 23.8 & 23.8 & 23.9 \\
\hline ESF-HD-175-TEMP28-RTD-42 & 10.53 & 39.23 & 10.74 & 15.04 & 23.5 & 23.5 & 23.5 & 23.5 & 23.5 & 23.5 & 23.6 & 23.6 & 23.7 & 23.7 \\
\hline ESF-HD-175-TEMP28-RTD-43 & 10.74 & 39.23 & 10.95 & 15.34 & 23.4 & 23.4 & 23.5 & 23.5 & 23.5 & 23.6 & 23.6 & 23.6 & 23.7 & 23.7 \\
\hline ESF-HD-175-TEMP28-RTD-44 & 10.95 & 39.23 & 11.17 & 15.64 & 23.4 & 23.4 & 23.5 & 23.5 & 23.2 & 23.5 & 23.5 & 23.6 & 23.6 & 23.7 \\
\hline ESF-HD-175-TEMP28-RTD-45 & 11.16 & 39.23 & 11.38 & 15.94 & 23.3 & 23.4 & 23.4 & 23.5 & 23.5 & 23.5 & 23.5 & 23.5 & 23.6 & 23.6 \\
\hline ESF-HD-175-TEMP28-RTD-46 & 11.37 & 39.22 & 11.60 & 16.24 & 23.4 & 23.4 & 23.4 & 23.4 & 23.5 & 23.5 & 23.5 & 23.5 & 23.5 & 23.6 \\
\hline ESF-HD-175-TEMP28-RTD-47 & 11.57 & 39.22 & 11.81 & 16.53 & 23.1 & 23.2 & 23.4 & 23.4 & 23.4 & 23.4 & 23.5 & 23.5 & 23.5 & 23.5 \\
\hline ESF-HD-175-TEMP28-RTD-48 & 11.78 & 39.22 & 12.03 & 16.84 & 23.3 & 23.3 & 23.4 & 23.4 & 23.4 & 23.5 & 23.5 & 23.5 & 23.5 & 23.5 \\
\hline ESF-HD-175-TEMP28-RTD-49 & 11.99 & 39.22 & 12.24 & 17.13 & 23.4 & 23.2 & 23.4 & 22.9 & 23.4 & 23.4 & 23.9 & 23.4 & 23.4 & 23.5 \\
\hline ESF-HD-175-TEMP28-RTD-50 & 12.20 & 39.21 & 12.46 & 17.44 & 23.4 & 23.4 & 23.4 & 23.4 & 23.4 & 23.4 & 23.4 & 23.4 & 23.5 & 23.5 \\
\hline ESF-HD-175-TEMP28-RTD-51 & 12.41 & 39.21 & 12.68 & 17.74 & 23.4 & 23.4 & 23.4 & 23.4 & 23.4 & 23.4 & 23.4 & 23.4 & 23.4 & 23.5 \\
\hline ESF-HD-175-TEMP28-RTD-52 & 12.62 & 39.21 & 12.89 & 18.04 & 23.2 & 23.3 & 23.4 & 23.4 & 23.4 & 23.4 & 23.4 & 23.4 & 23.4 & 23.4 \\
\hline ESF-HD-175-TEMP28-RTD-53 & 12.83 & 39.21 & 13.11 & 18.34 & 23.4 & 23.4 & 23.4 & 23.4 & 23.4 & 23.4 & 23.4 & 23.4 & 23.4 & 23.4 \\
\hline ESF-HD-175-TEMP28-RTD-54 & 13.04 & 39.21 & 13.32 & 18.64 & 23.4 & 23.4 & 23.4 & 23.4 & 23.4 & 23.3 & 23.4 & 23.4 & 23.4 & 23.4 \\
\hline ESF-HD-175-TEMP28-RTD-55 & 13.25 & 39.20 & 13.54 & 18.94 & 23.4 & 23.4 & 23.4 & 23.4 & 23.4 & 23.4 & 23.4 & 23.4 & 23.4 & 23.4 \\
\hline ESF-HD-175-TEMP28-RTD-56 & 13.46 & 39.20 & 13.75 & 19.24 & 23.3 & 23.3 & 23.3 & 23.4 & 23.4 & 23.4 & 23.4 & 23.4 & 23.4 & 23.4 \\
\hline ESF-HD-175-TEMP28-RTD-57 & 13.67 & 39.20 & 13.97 & 19.55 & 23.4 & 23.4 & 23.4 & 23.4 & 23.4 & 23.4 & 23.4 & 23.4 & 23.4 & 23.4 \\
\hline ESF-HD-175-TEMP28-RTD-58 & 13.88 & 39.20 & 14.18 & 19.84 & 23.3 & 23.4 & 23.4 & 23.4 & 23.4 & 23.4 & 23.4 & 23.4 & 23.4 & 23.4 \\
\hline ESF-HD-175-TEMP28-RTD-59 & 14.09 & 39.20 & 14.40 & 20.15 & 23.1 & 23.2 & 23.3 & 23.3 & 23.3 & 23.3 & 23.3 & 23.3 & 23.3 & 23.3 \\
\hline ESF-HD-175-TEMP28-RTD-60 & 14.30 & 39.19 & 14.61 & 20.44 & 23.4 & 23.4 & 23.4 & 23.4 & 23.4 & 23.4 & 23.4 & 23.4 & 23.4 & 23.4 \\
\hline ESF-HD-175-TEMP28-RTD-61 & 14.51 & 39.19 & 14.83 & 20.75 & 23.3 & 23.2 & 23.3 & 23.3 & 23.3 & 23.3 & 23.3 & 23.3 & 23.3 & 23.3 \\
\hline
\end{tabular}


First Quarter Report of Temperature Measurements During the Heating Phase of the Drift-Scale Test

\begin{tabular}{|c|c|c|c|c|c|c|c|c|c|c|c|c|c|c|}
\hline & \multicolumn{14}{|c|}{ Temperature Data Obtained from the DST } \\
\hline & \multicolumn{14}{|c|}{ Days after startup } \\
\hline & \multicolumn{4}{|c|}{ As Built Locations (m) } & 0 & 7 & 17 & 27 & 38 & 48 & 58 & 69 & 79 & 87 \\
\hline & $\mathbf{x}$ & y & $\mathbf{z}$ & D & \multicolumn{10}{|c|}{$\left({ }^{\circ} \mathrm{C}\right)$} \\
\hline ESF-HD-175-TEMP28-RTD-62 & 14.72 & 39.19 & 15.05 & 21.05 & 23.3 & 23.3 & 23.3 & 23.3 & 23.3 & 23.3 & 23.3 & 23.3 & 23.3 & 23.3 \\
\hline ESF-HD-175-TEMP28-RTD-63 & 14.93 & 39.19 & 15.26 & 21.35 & 23.3 & 23.3 & 23.3 & 23.3 & 23.3 & 23.3 & 23.3 & 23.3 & 23.3 & 23.3 \\
\hline ESF-HD-175-TEMP28-RTD-64 & 15.14 & 39.18 & 15.48 & 21.65 & 22.9 & 22.9 & 23.1 & 23.1 & 21.8 & 22.2 & 23.0 & 23.3 & 23.3 & 23.3 \\
\hline ESF-HD-175-TEMP28-RTD-65 & 15.35 & 39.18 & 15.69 & 21.95 & 23.3 & 23.3 & 23.3 & 23.3 & 23.3 & 23.3 & 23.3 & 23.3 & 23.3 & 23.3 \\
\hline ESF-HD-175-TEMP28-RTD-66 & 15.56 & 39.18 & 15.91 & 22.25 & 23.3 & 23.3 & 23.3 & 23.3 & 23.3 & 23.3 & 23.3 & 23.3 & 23.3 & 23.3 \\
\hline ESF-HD-175-TEMP28-RTD-67 & 15.77 & 39.18 & 16.12 & 22.55 & 23.3 & 23.3 & 23.3 & 23.3 & 23.3 & 23.3 & 23.3 & 23.3 & 23.3 & 23.3 \\
\hline
\end{tabular}


Appendix B 



\section{Appendix B}

Revised datum assignments for borehole 80

\begin{tabular}{|l|l|}
\hline \multicolumn{2}{|c|}{ Borehole 80 } \\
\hline & $\begin{array}{c}\text { Recommended } \\
\text { Datum }\end{array}$ \\
\hline \hline ESF-HD-80-TEMP2-RTD-1 & RTD-2 \\
\hline ESF-HD-80-TEMP2-RTD-2 & RTD-1 \\
\hline ESF-HD-80-TEMP2-RTD-3 & RTD-4 \\
\hline ESF-HD-80-TEMP2-RTD-4 & RTD-3 \\
\hline ESF-HD-80-TEMP2-RTD-5 & RTD-6 \\
\hline ESF-HD-80-TEMP2-RTD-6 & RTD-5 \\
\hline ESF-HD-80-TEMP2-RTD-7 & RTD-8 \\
\hline ESF-HD-80-TEMP2-RTD-8 & RTD-7 \\
\hline ESF-HD-80-TEMP2-RTD-9 & RTD-10 \\
\hline ESF-HD-80-TEMP2-RTD-10 & RTD-9 \\
\hline ESF-HD-80-TEMP2-RTD-11 & RTD-12 \\
\hline ESF-HD-80-TEMP2-RTD-12 & RTD-11 \\
\hline ESF-HD-80-TEMP2-RTD-13 & RTD-14 \\
\hline ESF-HD-80-TEMP2-RTD-14 & RTD-13 \\
\hline ESF-HD-80-TEMP2-RTD-15 & RTD-16 \\
\hline ESF-HD-80-TEMP2-RTD-16 & RTD-15 \\
\hline ESF-HD-80-TEMP2-RTD-17 & RTD-18 \\
\hline ESF-HD-80-TEMP2-RTD-18 & RTD-17 \\
\hline ESF-HD-80-TEMP2-RTD-19 & RTD-20 \\
\hline ESF-HD-80-TEMP2-RTD-20 & RTD-19 \\
\hline ESF-HD-80-TEMP2-RTD-21 & RTD-22 \\
\hline ESF-HD-80-TEMP2-RTD-22 & RTD-21 \\
\hline ESF-HD-80-TEMP2-RTD-23 & RTD-24 \\
\hline ESF-HD-80-TEMP2-RTD-24 & RTD-23 \\
\hline
\end{tabular}



Appendix C 



\section{Appendix C}

Revised locations for RTD sensors in borehole 134

\begin{tabular}{|c|c|c|c|}
\hline \multicolumn{4}{|c|}{ ESF-HD-134 } \\
\hline & $\mathbf{x}$ & $\mathbf{y}$ & $\mathbf{z}$ \\
\hline ESF-HD-134-TEMP4-RTD-1 & 0.74 & 2.73 & -1.85 \\
\hline ESF-HD-134-TEMP4-RTD-2 & 0.74 & 2.73 & -2.16 \\
\hline ESF-HD-134-TEMP4-RTD-3 & 0.74 & 2.73 & -2.48 \\
\hline ESF-HD-134-TEMP4-RTD-4 & 0.73 & 2.73 & -2.80 \\
\hline ESF-HD-134-TEMP4-RTD-5 & 0.73 & 2.74 & -3.11 \\
\hline ESF-HD-134-TEMP4-RTD-6 & 0.73 & 2.74 & -3.43 \\
\hline ESF-HD-134-TEMP4-RTD-7 & 0.73 & 2.74 & -3.74 \\
\hline ESF-HD-134-TEMP4-RTD-8 & 0.73 & 2.74 & -4.06 \\
\hline ESF-HD-134-TEMP4-RTD-9 & 0.73 & 2.74 & -4.37 \\
\hline ESF-HD-134-TEMP4-RTD-10 & 0.73 & 2.74 & -4.69 \\
\hline ESF-HD-134-TEMP4-RTD-11 & 0.72 & 2.74 & -5.01 \\
\hline ESF-HD-134-TEMP4-RTD-12 & 0.72 & 2.74 & -5.32 \\
\hline ESF-HD-134-TEMP4-RTD-13 & 0.72 & 2.74 & -5.64 \\
\hline ESF-HD-134-TEMP4-RTD-14 & 0.72 & 2.74 & -5.95 \\
\hline ESF-HD-134-TEMP4-RTD-15 & 0.72 & 2.74 & -6.27 \\
\hline ESF-HD-134-TEMP4-RTD-16 & 0.72 & 2.74 & -6.58 \\
\hline ESF-HD-134-TEMP4-RTD-17 & 0.72 & 2.74 & -6.9 \\
\hline ESF-HD-134-TEMP4-RTD-18 & 0.71 & 2.75 & -7.22 \\
\hline ESF-HD-134-TEMP4-RTD-19 & 0.71 & 2.75 & -7.53 \\
\hline ESF-HD-134-TEMP4-RTD-20 & 0.71 & 2.75 & -7.85 \\
\hline ESF-HD-134-TEMP4-RTD-21 & 0.71 & 2.75 & -8.16 \\
\hline ESF-HD-134-TEMP4-RTD-22 & 0.71 & 2.75 & -8.48 \\
\hline ESF-HD-134-TEMP4-RTD-23 & 0.71 & 2.75 & -8.79 \\
\hline ESF-HD-134-TEMP4-RTD-24 & 0.70 & 2.75 & -9.11 \\
\hline ESF-HD-134-TEMP4-RTD-25 & 0.70 & 2.75 & -9.43 \\
\hline ESF-HD-134-TEMP4-RTD-26 & 0.70 & 2.75 & -9.74 \\
\hline ESF-HD-134-TEMP4-RTD-27 & 0.70 & 2.75 & -10.06 \\
\hline ESF-HD-134-TEMP4-RTD-28 & 0.70 & 2.75 & -10.37 \\
\hline ESF-HD-134-TEMP4-RTD-29 & 0.70 & 2.75 & -10.69 \\
\hline ESF-HD-134-TEMP4-RTD-30 & 0.70 & 2.75 & -11.00 \\
\hline ESF-HD-134-TEMP4-RTD-31 & 0.69 & 2.76 & -11.32 \\
\hline
\end{tabular}


First Quarter Report of Temperature Measurements

During the Heating Phase of the Drift-Scale Test

\begin{tabular}{|c|c|c|c|}
\hline \multicolumn{4}{|c|}{ ESF-HD-134 } \\
\hline & $\mathbf{x}$ & y & $\mathbf{z}$ \\
\hline ESF-HD-134-TEMP4-RTD-32 & 0.69 & 2.76 & -11.64 \\
\hline ESF-HD-134-TEMP4-RTD-33 & 0.69 & 2.76 & -11.95 \\
\hline ESF-HD-134-TEMP4-RTD-34 & 0.69 & 2.76 & -12.27 \\
\hline ESF-HD-134-TEMP4-RTD-35 & 0.69 & 2.76 & -12.58 \\
\hline ESF-HD-134-TEMP4-RTD-36 & 0.69 & 2.76 & -12.9 \\
\hline ESF-HD-134-TEMP4-RTD-37 & 0.69 & 2.76 & -13.21 \\
\hline ESF-HD-134-TEMP4-RTD-38 & 0.68 & 2.76 & -13.53 \\
\hline ESF-HD-134-TEMP4-RTD-39 & 0.68 & 2.76 & -13.85 \\
\hline ESF-HD-134-TEMP4-RTD-40 & 0.68 & 2.76 & -14.16 \\
\hline ESF-HD-134-TEMP4-RTD-41 & 0.68 & 2.76 & -14.48 \\
\hline ESF-HD-134-TEMP4-RTD-42 & 0.68 & 2.76 & -14.79 \\
\hline ESF-HD-134-TEMP4-RTD-43 & 0.68 & 2.77 & -15.11 \\
\hline ESF-HD-134-TEMP4-RTD-44 & 0.67 & 2.77 & -15.42 \\
\hline ESF-HD-134-TEMP4-RTD-45 & 0.67 & 2.77 & -15.74 \\
\hline ESF-HD-134-TEMP4-RTD-46 & 0.67 & 2.77 & -16.06 \\
\hline ESF-HD-134-TEMP4-RTD-47 & 0.67 & 2.77 & -16.37 \\
\hline ESF-HD-134-TEMP4-RTD-48 & 0.67 & 2.77 & -16.69 \\
\hline ESF-HD-134-TEMP4-RTD-49 & 0.67 & 2.77 & -17.00 \\
\hline ESF-HD-134-TEMP4-RTD-50 & 0.67 & 2.77 & -17.32 \\
\hline ESF-HD-134-TEMP4-RTD-51 & 0.66 & 2.77 & -17.63 \\
\hline ESF-HD-134-TEMP4-RTD-52 & 0.66 & 2.77 & -17.95 \\
\hline ESF-HD-134-TEMP4-RTD-53 & 0.66 & 2.77 & -18.27 \\
\hline ESF-HD-134-TEMP4-RTD-54 & 0.66 & 2.77 & -18.58 \\
\hline ESF-HD-134-TEMP4-RTD-55 & 0.66 & 2.77 & -18.90 \\
\hline ESF-HD-134-TEMP4-RTD-56 & 0.66 & 2.78 & -19.21 \\
\hline ESF-HD-134-TEMP4-RTD-57 & 0.66 & 2.78 & -19.53 \\
\hline ESF-HD-134-TEMP4-RTD-58 & 0.65 & 2.78 & -19.84 \\
\hline ESF-HD-134-TEMP4-RTD-59 & 0.65 & 2.78 & -20.16 \\
\hline ESF-HD-134-TEMP4-RTD-60 & 0.65 & 2.78 & -20.79 \\
\hline ESF-HD-134-TEMP4-RTD-61 & 0.65 & 2.78 & -21.11 \\
\hline ESF-HD-134-TEMP4-RTD-62 & 0.65 & 2.78 & -21.42 \\
\hline ESF-HD-134-TEMP4-RTD-63 & 0.65 & 2.78 & -21.74 \\
\hline ESF-HD-134-TEMP4-RTD-64 & 0.64 & 2.78 & -22.05 \\
\hline ESF-HD-134-TEMP4-RTD-65 & 0.64 & 2.78 & -22.37 \\
\hline ESF-HD-134-TEMP4-RTD-66 & 0.64 & 2.78 & -22.69 \\
\hline
\end{tabular}




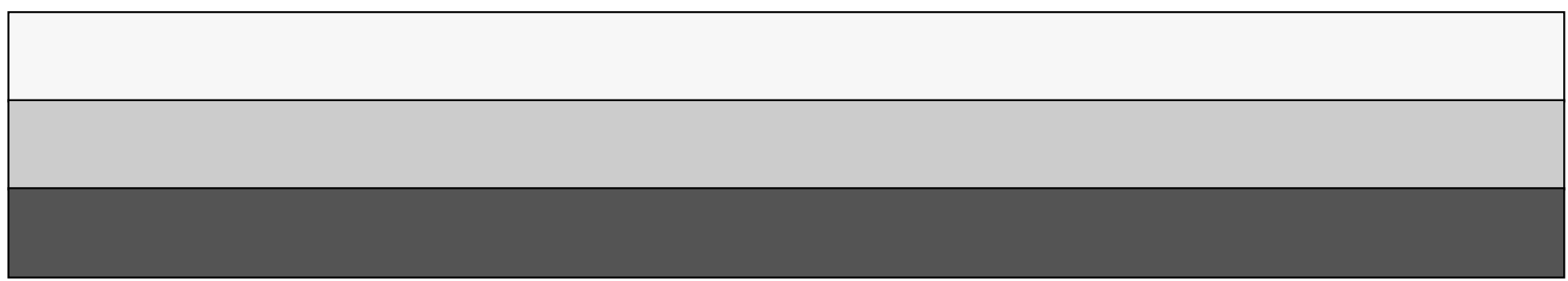

